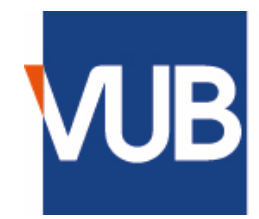

VRIJE

UNIVERSITEIT

BRUSSEL

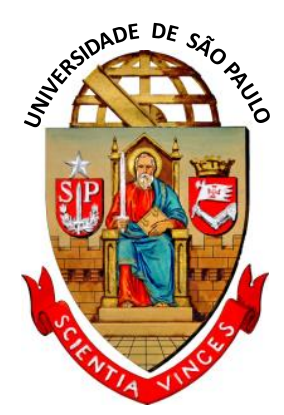

\title{
NEXT GENERATION ACCESS NETWORKS: FLEXIBLE OCDMA SYSTEMS AND COST- EFFECTIVE CHAOTIC VCSEL SOURCES FOR SECURE COMMUNICATIONS
}

Doctoral thesis submitted in fulfilment of the requirements for the academic degree of Doctor in Engineering and Doctor in Science by

\section{THIAGO R. RADDO}

August 2017

Promotors: Prof. Dr. Ir. Heidi Ottevaere (VUB)

Prof. Dr. Ben-Hur Viana Borges (USP)

Dr. Martin Virte (VUB)

Vrije Universiteit Brussel

Department of Applied Physics and Photonics

Brussels Photonics, B-PHOT

University of São Paulo

São Carlos School of Engineering

Department of Electrical and Computer Engineering 


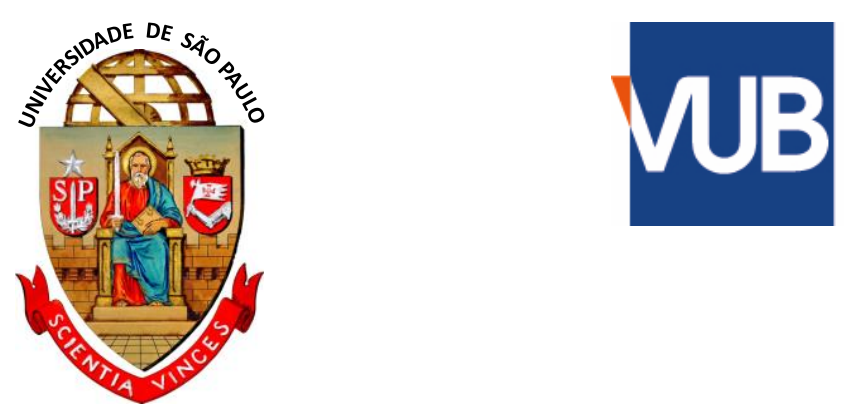

VRIJE

UNIVERSITEIT BRUSSEL

\section{REDES DE ACESSO DE PRÓXIMA GERAÇÃO: SISTEMAS OCDMA FLEXÍVEIS E FONTES VCSEL CAÓTICAS DE BAIXO CUSTO PARA COMUNICAÇÕES SEGURAS}

Tese apresentada em cumprimento dos requisitos para o grau acadêmico de Doutor em Ciências, Programa de Engenharia Elétrica, Telecomunicações, por

\section{THIAGO R. RADDO}

Agosto 2017

\section{Orientadores: Prof. Dr. Ben-Hur Viana Borges (USP) \\ Prof. Dr. Ir. Heidi Ottevaere (VUB) \\ Dr. Martin Virte (VUB)}

Universidade de São Paulo

Escola de Engenharia de São Carlos

Departamento de Engenharia Elétrica e de Computação

Vrije Universiteit Brussel

Department of Applied Physics and Photonics

Brussels Photonics, B-PHOT

Trata-se da versão original da tese 


\section{AUTORIZO A REPRODUÇÃO TOTAL OU PARCIAL DESTE TRABALHO, POR QUALQUER MEIO CONVENCIONAL OU ELETRÔNICO, PARA FINS DE ESTUDO E PESQUISA, DESDE QUE CITADA A FONTE.}

Raddo, Thiago Roberto

R124r

Redes de acesso de próxima geração: sistemas OCDMA flexíveis e fontes VCSEL caóticas de baixo custo para comunicações seguras = Next generation access networks: flexible OCDMA systems and cost-effective chaotic VCSEL sources for secure communications / Thiago Roberto Raddo; orientador Ben-Hur Viana Borges; coorientadora Heidi ottevaere. São Carlos, 2017.

Tese (Doutorado) - Programa de Pós-Graduação em Engenharia Elétrica e Área de Concentração em Telecomunicações -- Escola de Engenharia de São Carlos da Universidade de São Paulo, 2017.

1. Acesso múltiplo por divisão de código óptico. 2 . Taxa de erro de bit. 3. Comutação de pacotes. 4. Múltiplas taxas. 5. Laser de emissão superficial com cavidade vertical. 6. Polarização caótica. 7. Caos. I. Título. 


\section{FOLHA DE JULGAMENTO}

Candidato: Tecnólogo THIAGO ROBERTO RADDO.

Título da tese: "Redes de acesso de próxima geração: sistemas OCDMA flexíveis e fontes caóticas de baixo custo para comunicaçōes seguras".

Data da defesa: 22/08/2017.

Comissão Julgadora:

Prof. Associado Ben-Hur Viana Borges

(Orientador)

(Escola de Engenharia de São Carlos/EESC)

Profa. Dra. Mônica de Lacerda Rocha

(Escola de Engenharia de São Carlos/EESC)

Prof. Dr. Heidi Ottevaere

(Vrije Universiteit Brusseis/VUB)

Prof. Dr. Idelfonso Tafur Monroy

(Technical University of Denmark)

Prof. Dr. Stefan Brever

(Technische Universität Darmstadt)

Prof. Dr. Martin Virte

(Vrije Universiteit Brusseis/VUB)
Resultado:

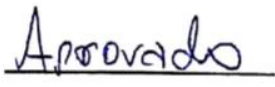

Aprovado

Aprovado

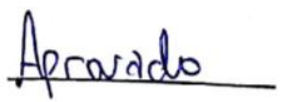

Afrovado

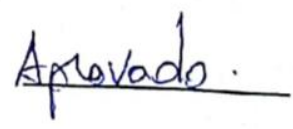

Coordenador do Programa de Pós-Graduação em Engenharia Elétrica: Prof. Associado Luis Fernando Costa Alberto

Presidente da Comissão de Pós-Graduação:

Prof. Associado Luis Fernando Costa Alberto 

To my beloved parents, Roberto and Abgail 



\section{Members of the Jury}

Prof. Dr. Ir. Heidi Ottevaere, promotor Department of Applied Physics and Photonics

Vrije Universiteit Brussel, Belgium

Prof. Dr. Ben-Hur Viana Borges, promotor Department of Electrical and Computer Engineering University of São Paulo, Brazil

Dr. Martin Virte, promotor Department of Applied Physics and Photonics

Vrije Universiteit Brussel, Belgium

Prof. Dr. Ir. Hugo Thienpont, secretary Department of Applied Physics and Photonics

Vrije Universiteit Brussel, Belgium

Prof. Dr. Ir. Peter Schelkens, chair Department of Electronics and Informatics

Vrije Universiteit Brussel, Belgium

Prof. Dr. Roger Vounckx, vice-chair Department of Electronics and Informatics

Vrije Universiteit Brussel, Belgium

Prof. Dr. Idelfonso Tafur Monroy Department of Photonics Engineering Technical University of Denmark, Denmark

Priv.-Doz. Dr. Habil. Stefan Breuer Institut für Angewandte Physik Technische Universität Darmstadt, Germany

Prof. Dr. Mônica de Lacerda Rocha Department of Electrical and Computer Engineering University of São Paulo, Brazil 



\section{Acknowledgements}

Firstly, I would like to thank God for everything in life. I would like to thank my parents, Roberto Raddo and Abgail Raddo as well as my girlfriend Anne-Sophie for their constant support, love, encouragement, and total understanding during my long doctoral journey.

I would like to thank Ben-Hur V. Borges, Heidi Ottevaere, Idelfonso T. Monroy, and Martin Virte for their contributions to my doctoral research as my academic advisors. I am grateful for their continuous guidance during this long and challenging path, for their helpfulness, confidence, and advice. Special thanks go to Hugo Thienpont and Heidi Ottevaere for the unique offered opportunity to develop part of the doctoral research in Brussels at B-PHOT.

I would like to express my gratitude to my great friends:

Israel Lot (thanks also for the book's cover), Larissa Lima (thanks also for helping me out so many times), Andrea Ceglia, Antonio Navas, Alex Ferreira, Daniel Marchesi, Dana Szafranek, Igor Feliciano, Indayara Martins, Valdemir dos Reis, Thiago Campos, Thiago Franco, and Anderson Sanches.

Thanks to my friends from B-PHOT VUB: Achiles, Agnieszka, Ana, Ben de Pauw, Benjamin, Etienne, Evert, Fabian, Fei, Felicity, Gebirie, Giancarlo, Iterio, Jens, Koen, Lara, Lien, Majorie, Marcin, Mulham, Qing, Raymundo, Robert, Sanne, Sid, Simone, Sophie, Tigran, Urszula, Yunfeng, and ZuChen. You guys rock and I miss you all already. I just want to send you all the biggest thanks!

Finally, thanks to my friends from DTU: Ana, Bruno, Christoph, Jesper, Lau, Łukasz, Lucas, Nicklas, Peter, Rafael, Silvia, Simon, Victor, and Xiaofeng.

The generous funding of this doctoral research by CAPES and FWO Flanders agencies is gratefully acknowledged.

Thiago Raddo

Brussels, 2017 



\section{Resumo}

RADDO., T. R. Redes de Acesso de Próxima Geração: Sistemas OCDMA Flexíveis e Fontes VCSEL Caóticas de Baixo Custo para Comunicações Seguras. Tese (Doutorado), Escola de Engenharia de São Carlos, Universidade de São Paulo, São Carlos, 2017.

Os avanços relacionados a tecnologia fotônica ampliaram o alcance das redes de comunicação óptica tanto em instalações de estabelecimentos comerciais quanto em residências, permitindo que novos serviços e tecnologias fossem entregues aos clientes. A próxima onda de inovação certamente gerará inúmeras oportunidades proporcionadas pela popularidade de soluções emergentes e aplicações como a Internet tátil, a telemedicina e a geração de conteúdo 3-D em tempo real, tornandoos parte da vida cotidiana. No entanto, para suportar a crescente demanda de tráfego atual, uma maior capacidade e segurança, alocação flexível de largura de banda e custo-eficiência tornaram-se requisitos cruciais para as tecnologias candidatas a futuras redes de acesso óptico. Para este fim, a tecnologia de acesso múltiplo por divisão de código óptico (OCDMA) é considerada um candidato em potencial, particularmente devido a características como transmissões assíncronas, distribuição flexível de banda larga e suporte a serviços diferenciados na camada física, para citar apenas alguns. Neste contexto, esta tese propõe novos formalismos matemáticos para a taxa de erro de bits, taxa de transferência de pacotes e atraso de pacotes para avaliar o desempenho de redes OCDMA flexíveis capazes de fornecer transmissões em múltiplas qualidades de serviço (QoS) de acordo com as necessidades dos usuários. Os formalismos analíticos propostos não requerem o conhecimento a priori das sequências de código dos usuários, o que significa que o desempenho da rede pode ser abordado de forma simples e direta usando apenas os parâmetros de código. Além disso, os formalismos analíticos desenvolvidos representam um número geral de classes de usuários distintos, bem como a probabilidade geral de interferência entre os usuários. Portanto, esses formalismos podem ser aplicados com sucesso na avaliação de desempenho de redes OCDMA flexíveis não apenas em qualquer número de classes de usuários em uma rede, mas também para a maioria dos códigos de espalhamento com boas propriedades de correlação. A expressão de taxa de transferência de pacotes é derivada assumindo aproximações de Poisson, binomial e de cadeia de Markov para as chegadas de pacotes compostos, com a última definida como benchmark. Em seguida, é mostrado via simulação numérica que a expressão baseada em Poisson não é apropriada para uma estimativa confiável de taxa de transferência quando comparada aos resultados de benchmark (Markov). A equação de taxa de transferência binomial, por sua vez, fornece resultados tão precisos quanto o benchmark. Além disso, a taxa de transferência binomial é numericamente mais conveniente e computacionalmente eficiente quando comparada com abordagem de 
Markov, enquanto esta última é computacionalmente dispendiosa, particularmente se o número de usuários é grande. As expressões de taxa de erro de bit (BER) são derivadas considerando distribuições gaussianas e binomiais para a interferência de acesso múltiplo e é mostrado por meio de simulações numéricas que o desempenho exato de redes OCDMA flexíveis é obtido somente com a expressão binomial de BER. Esta tese também propõe e investiga uma arquitetura de rede para o tráfego de protocolo de Internet sobre OCDMA flexível com suporte a transmissões de QoS e de múltiplas taxas, que é independente do código de espalhamento empregado e não requer qualquer nova tecnologia de processamento óptico. Além disso, o desempenho da rede assume que os usuários transmitem de forma assíncrona usando receptores baseados em esquemas de detecção direta de modulação de intensidade. As simulações numéricas mostraram que a rede proposta possui melhor desempenho quando seus usuários são definidos com peso de código alto ou quando a utilização do canal é baixa. O desempenho da BER e da taxa de transferência de pacotes de uma rede OCDMA que fornece transmissões de múltiplas taxas por meio de uma técnica multi-código com dois códigos atribuídos a cada usuário é também abordado. Os resultados numéricos mostram que esta técnica supera as técnicas clássicas baseadas no código de comprimento múltiplo. Finalmente, esta tese aborda uma nova tecnologia que pode levar a níveis mais elevados de segurança na camada física de redes ópticas. Esta tecnologia consiste na geração de caos determinístico a partir de um laser de emissão superficial com cavidade vertical (VCSEL). A dinâmica caótica é gerada através da aplicação de forças mecânicas em um VCSEL comercial usando um suporte simples e facilmente replicável. O caos determinístico é então alcançado, pela primeira vez, sem qualquer complexidade adicional de realimentação óptica, modulação de parâmetros ou injeção óptica. A simplicidade do sistema proposto, o qual se baseia inteiramente em componentes de baixo custo e que são facilmente encontrados, abre o caminho para o uso de dispositivos VCSEL comerciais para aplicações baseadas em caos. Este gerador de caos óptico tem o potencial não só de pavimentar o caminho para novas plataformas de segurança em redes ópticas, como, por exemplo, ocultar com êxito as informações do usuário em um sinal imprevisível e aleatório contra eventuais invasores, como também tem o potencial de influenciar aplicações de caos emergentes inicialmente limitadas ou inviáveis devido à falta de soluções de baixo custo. Além disso, ele conduz o caminho para a realização futura de aplicações emergentes com alta integridade e escalabilidade, tais como matrizes bidimensionais de dispositivos caóticos que compreendem centenas de fontes individuais para aumentar as necessidades de geração de bit aleatória, criptografia ou redes quânticas de grande escala.

Palavras-chave: acesso múltiplo por divisão de código óptico, taxa de erro de bit, taxa de transferência, comutação de pacotes, flexível, elástica, serviços diferenciados, múltiplas taxas, laser de emissão superficial com cavidade vertical, funcionamento livre, laser díodo, polarização caótica, caos. 


\section{Samenvatting}

De aanzienlijke vooruitgang in glasvezeltechnologie heeft het bereik van het optische netwerk bij eindgebruikers in bedrijfspanden en zelfs huizen uitgebreid, waardoor nieuwe diensten en technologieën geleverd kunnen worden aan de klanten. Door de enorme populariteit van nieuwe oplossingen en toepassingen, zoals het tactiele internet, telegeneeskunde en real-time 3-D inhoudsgeneratie, zullen er in de toekomst talrijke mogelijkheden gegenereerd worden om de kwaliteit van ons dagelijks leven te verbeteren. Maar om een dergelijke verhoging van dataverkeer te ondersteunen, zijn hogere capaciteit en veiligheid, flexibele toewijzing van bandbreedte en kostenefficiëntie van cruciaal belang voor de technologieën in toekomstige optische netwerken. Optische code-division multiple-access (OCDMA) technologie is hiervoor een potentiële kandidaat, vanwege eigenschappen zoals asynchrone transmissies, flexibele en bewuste bandbreedte distributie en ondersteuning van gedifferentieerde diensten in de fysieke laag.

In dit proefschrift worden nieuwe wiskundige formalismen voor de bit error rate, de pakket doorvoer en pakket delay voorgesteld om de prestaties van flexibele OCDMA netwerken te beoordelen, die in staat zijn om multiservice, multirate transmissies volgens de noden van de gebruikers te leveren. De voorgestelde analytische formalismen vereisen geen 'a prioiri' kennis van de volgorde van de gebruikerscode, waardoor de prestaties van het netwerk op een eenvoudige en directe wijze kunnen worden aangepakt met alleen de code parameters. Bovendien nemen de ontwikkelde analytische formalismen een algemeen aantal onderscheidbare gebruikersklassen in rekening, alsook de kans op interferentie tussen gebruikers. Daarom kunnen deze formalismen met succes worden toegepast voor de evaluatie van de prestaties van flexibele OCDMA netwerken, niet alleen onder een aantal klassen van gebruikers in een netwerk, maar ook voor het grootste deel bij het verspreiden van codes met goede correlatie eigenschappen. De pakket doorvoer expressie wordt verkregen uitgaande van Poisson, binomiale en Markov keten aanpakken voor de samengestelde pakket aankomsten waarbij de Markov expressie wordt gebruikt als benchmark. Vervolgens wordt door numerieke simulaties aangetoond dat de Poisson-gebaseerde voorstelling niet geschikt is voor een betrouwbare schatting van doorvoercapaciteit in vergelijking met de referentie (Markov). De binomiaal-gebaseerde doorvoer vergelijking levert even nauwkeurige resultaten op als de referentie. Bovendien is de binomiaal-gebaseerde doorvoer numeriek handiger en rekenkundig efficiënter dan de Markov keten aanpak, terwijl de Markov-gebaseerde rekenkundig veeleisend is, vooral als het aantal gebruikers groot is. De bit error rate (BER) expressies worden afgeleid door naar Gaussiaanse en binomiale verdelingen te kijken voor meervoudige toegangsinterferentie en er wordt aangetoond via numerieke simulaties dat de 
nauwkeurige performantie van flexibele OCDMA-netwerken enkel verkregen wordt met de binomiaal-gebaseerde BER expressie.

Deze thesis onderzoekt en stelt ook een netwerkarchitectuur voor voor Internet Protocol verkeer over flexibele OCDMA, met ondersteuning voor multirate transmissies, die onafhankelijk zijn van de gebruikte spreidingscode en geen nieuwe optische technologie vereisen. Bovendien veronderstelt de netwerkprestatie dat gebruikers asynchroon zenden via receptoren op basis van de intensiteitsgemoduleerde directe detectie systemen. Numerieke simulaties tonen aan dat het voorgestelde netwerk goed presteert wanneer de gebruikers gedefinieerd zijn met een hoge gewichtscode of als het kanaal verbruik laag is. De BER en pakket doorvoer performantie van een OCDMA netwerk dat multirate transmissies verschaft via multicode techniek, met twee codes toegewezen aan elke gebruiker, wordt ook behandeld. Numerieke resultaten tonen dat deze techniek beter presteert dan klassieke technieken op basis van multilengte code.

Daarnaast richt dit proefschrift zich op een nieuwe baanbrekende technologie die zou kunnen leiden tot een hoger niveau van veiligheid in de fysieke laag van optische netwerken. Deze techniek bestaat uit het genereren van deterministische chaos vanuit een vertical-cavity surface-emitting laser (VCSEL). De chaotische dynamiek wordt opgewekt door mechanische spanningen op een commerciële off-the-shelf quantumwell VCSEL te zetten gebruik makende van een eenvoudige en gemakkelijk repliceerbare houder. Deterministische chaos wordt dan bereikt zonder aanvullende complexiteit van optische terugkoppeling, parametermodulatie of optische injectie. De eenvoud van het voorgestelde systeem, dat volledig gebaseerd is op goedkope en gemakkelijk beschikbare componenten, opent de weg naar het wijdverspreide gebruik van commerciële VCSEL devices voor chaos-gebaseerde applicaties. Deze off-the-shelf en kosteneffectieve optische chaos generator heeft het potentieel om niet alleen de weg vrij te maken voor nieuwe security platformen in optische netwerken, zoals bijvoorbeeld de gebruiker informatie te verbergen in een onvoorspelbaar, random-uitziend signaal als bescherming tegen eventuele afluisteraars, maar ook voor het beïnvloeden van nieuwe toepassingen gebaseerd op chaos, die vandaag beperkt of niet haalbaar zijn door het gebrek aan goedkope oplossingen. Bovendien opent dit doctoraatsonderzoek de weg naar de realisatie van nieuwe toepassingen met een hoge integreerbaarheid en schaalbaarheid zoals twee-dimensionale arrays van chaos-gebaseerde devices die honderden individuele bronnen bevatten om de eisen voor willekeurige bit generatie, cryptografie of grootschalige quantum netwerken te verhogen. 


\section{Summary}

RADDO., T. R. Next Generation Access Networks: Flexible OCDMA Systems and Cost-effective Chaotic VCSEL Sources for Secure Communications. 2017. Thesis (Ph.D.), São Carlos School of Engineering, University of São Paulo, São Carlos, 2017.

The significant advances in fiber-optic technology have broadened the optical network's reach into end-user business premises and even homes, allowing new services and technologies to be delivered to the customers. The next wave of innovation will certainly generate numerous opportunities provided by the widespread popularity of emerging solutions and applications such as tactile Internet, telemedicine and real time 3-D content generation, making them part of everyday life. Nevertheless, to support such an unprecedented and insatiable demand of data traffic, higher capacity and security, flexible bandwidth allocation and cost-efficiency have become crucial requirements for technologies candidate for future optical access networks. To this aim, optical code-division multiple-access (OCDMA) technology is considered as a prospective candidate, particularly due to features like asynchronous transmissions, flexible as well as conscious bandwidth resource distribution and support to differentiated services at the physical layer, to name but a few.

In this context, this thesis proposes new mathematical formalisms for bit error rate, packet throughput and packet delay to assess the performance of flexible OCDMA networks capable of providing multiservice multirate transmissions according to users' requirements. The proposed analytical formalisms do not require the knowledge a priori of the users' code sequences, which means that the network performance can be addressed in a simple and straightforward manner using the code parameters only. In addition, the developed analytical formalisms account for a general number of distinct users' classes as well as general probability of interference among users. Hence, these formalisms can be successfully applied for performance evaluation of flexible OCDMA networks not only under any number of users' classes in a network, but also for most spreading codes with good correlation properties. The packet throughput expression is derived assuming Poisson, binomial and Markov chain approaches for the composite packet arrivals with the latter defined as benchmark. Then, it is shown via numerical simulation the Poisson-based expression is not appropriate for a reliable throughput estimate when compared to the benchmark (Markov) results. The binomial-based throughput equation, by its turn, provides results as accurate as the benchmark. In addition, the binomial-based throughput is numerically more convenient and computationally more efficient than the Markov chain approach, whereas the Markov-based one is computationally 
expensive, particularly if the number of users is large. The bit error rate (BER) expressions are derived considering gaussian and binomial distributions for the multiple-access interference and it is shown via numerical simulations that accurate performance of flexible OCDMA networks is only obtained with the binomial-based BER expression.

This thesis also proposes and investigates a network architecture for Internet protocol traffic over flexible OCDMA with support to multiservice multirate transmissions, which is independent of the employed spreading code and does not require any new optical processing technology. In addition, the network performance assumes users transmitting asynchronously using receptors based on intensity-modulation directdetection schemes. Numerical simulations shown that the proposed network performs well when its users are defined with high-weight code or when the channel utilization is low. The BER and packet throughput performance of an OCDMA network that provides multirate transmissions via multicode technique with two codes assigned to each single user is also addressed. Numerical results show that this technique outperforms classical techniques based on multilength code.

Finally, this thesis addresses a new breakthrough technology that might lead to higher levels of security at the physical layer of optical networks. This technology consists in the generation of deterministic chaos from a commercial free-running vertical-cavity surface-emitting laser (VCSEL). The chaotic dynamics is generated by means of mechanical strains loaded onto an off-the-shelf quantum-well VCSEL using a simple and easily replicable holder. Deterministic chaos is then achieved, for the first time, without any additional complexity of optical feedback, parameter modulation or optical injection. The simplicity of the proposed system, which is based entirely on low-cost and easily available components, opens the way to the widespread use of commercial and free-running VCSEL devices for chaos-based applications. This off-the-shelf and cost-effective optical chaos generator has the potential for not only paving the way towards new security platforms in optical networks like, for example, successfully hiding the user information in an unpredictable, random-like signal against eventual eavesdroppers, but also for influencing emerging chaos applications initially limited or infeasible due to the lack of low-cost solutions. Furthermore, it leads the way to future realization of emerging applications with high-integrability and -scalability such as two-dimensional arrays of chaotic devices comprising hundreds of individual sources to increase requirements for random bit generation, cryptography or large-scale quantum networks.

Key-words: optical code-division multiple-access, bit error rate, packet throughput, packet network, flexible, elastic, multiservice, multirate, VCSEL, free-running, laser diode, polarization chaos, chaos. 


\title{
Table of Contents
}

\author{
ACKNOWLEDGEMENTS
}

RESUMO

III

SAMENVATTING

SUMMARY

VII

TABLE OF CONTENTS

IX

CHAPTER 1 INTRODUCTION 1

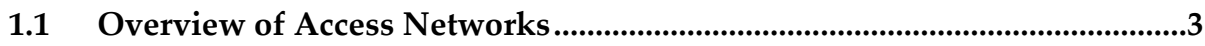

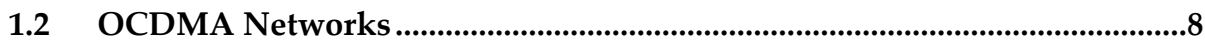

1.3 Towards Chaos Communications ................................................................15

1.4 Objectives and Organization of the Thesis ................................................18

CHAPTER 2 FLEXIBLE OCDMA NETWORKS 21

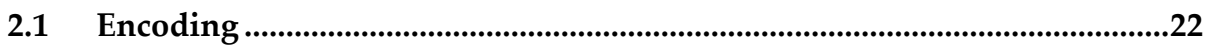

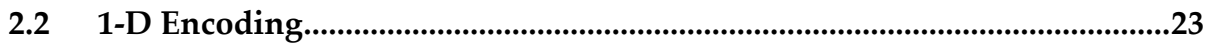

2.2.1 Encoding in Time Domain, 1-D MWML-OOC ........................................24

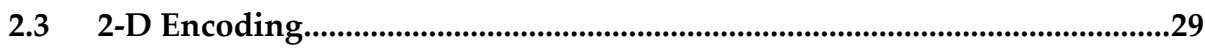

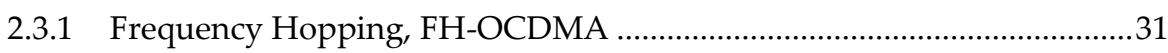

2.3.2 Fast Frequency Hopping, FFH-OCDMA ...............................................34

2.4 Multiple Access Interference ................................................................................39

2.5 Multirate Techniques ................................................................................................ 


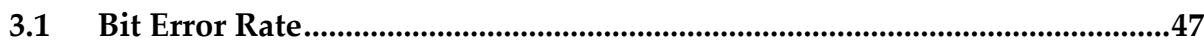

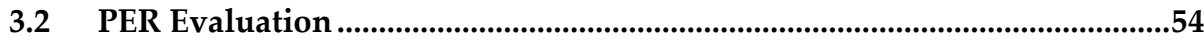

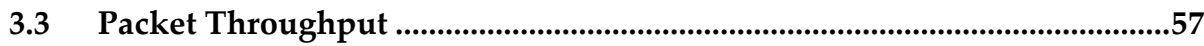

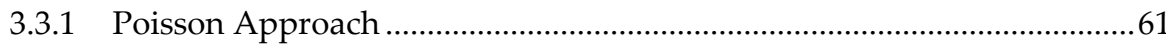

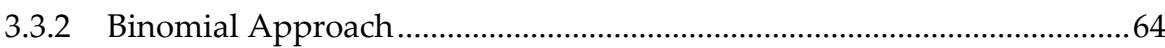

3.3.3 Markov Chain Approach ………................................................................65

3.3.4 Packet Throughput for Multicode Technique .........................................67

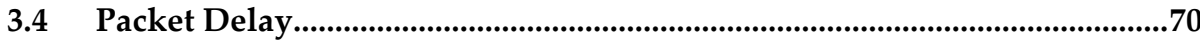

CHAPTER 4 NUMERICAL RESULTS AND DISCUSSIONS 73

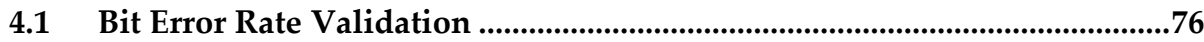

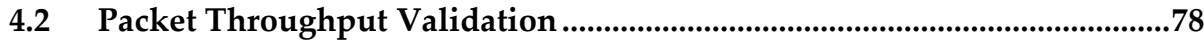

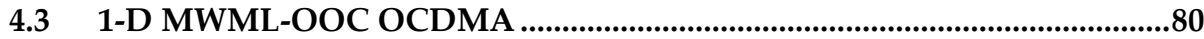

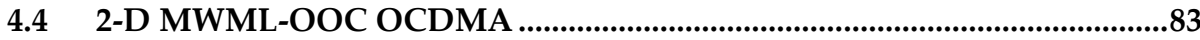

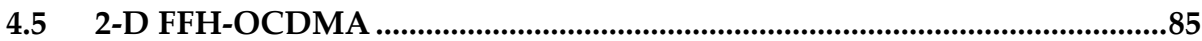

4.6 2-D MWML-OOC OCDMA, Multicode Technique ........................................90

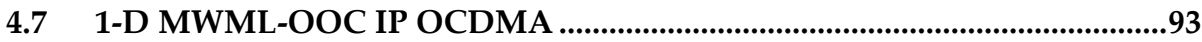

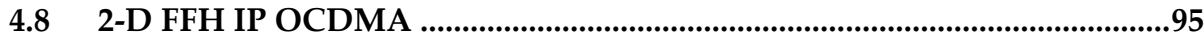

4.9 2-D MWML-OOC OCDMA, Packet Delay …..............................................99

CHAPTER 5 POLARIZATION CHAOS FROM FREE-RUNNING QW-VCSELS 103

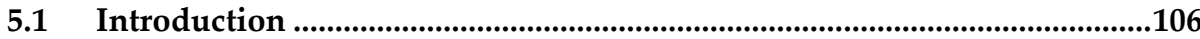

5.1.1 Chaos and its Potential for Secure Networks ........................................106

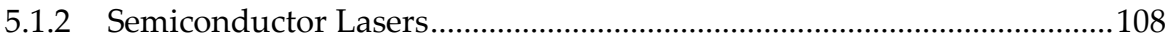

5.1.3 Polarization Instabilities and Chaos in VCSELs ....................................109

5.2 Experimental Setup and Mechanical Strain Technique .............................113

$5.3 \quad$ Experimental Chaos Identification ...................................................................116 


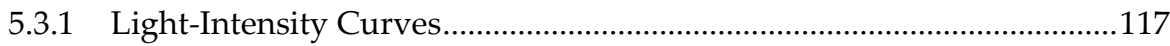

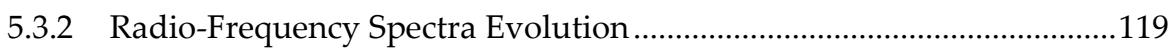

5.3.3 Time-Series and Random Like-Hopping .............................................120

5.4 Polarization Chaos Peculiarities..................................................................124

5.4.1 Light-Intensity Curves with an Iris Diaphragm ....................................124

5.4.2 Polarization and Frequency-Resolved Optical Spectra..........................128

5.4.3 Orientation of the Polarization Modes ....................................................130

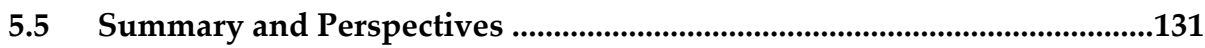

CHAPTER 6 CONCLUSIONS AND PERSPECTIVES 135

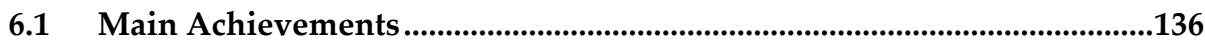

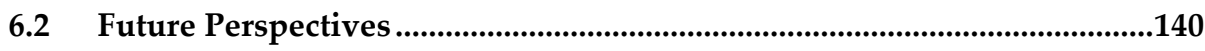

6.3 Summary of the Original Contributions ....................................................144

$\begin{array}{ll}\text { LIST OF PUBLICATIONS } & 147\end{array}$

$\begin{array}{ll}\text { LIST OF ACRONYMS } & 151\end{array}$

$\begin{array}{ll}\text { LIST OF SYMBOLS } & 155\end{array}$

$\begin{array}{ll}\text { LIST OF FIGURES } & 159\end{array}$

$\begin{array}{ll}\text { BIBLIOGRAPHY } & 167\end{array}$ 



\section{Chapter 1 Introduction}

This chapter covers general aspects of access networks and chaos dynamics generation in optical communication, with a brief overview of current solutions for broadband access and problems faced by today's technologies. Current technologies are facing a race against time for attending the ever-increasing demand for larger bandwidth of today's information society, in which instantaneous and reliable online access to any desired information has become primordial. In this context, fiber-to-thehome (FTTH) technology is becoming the dominant solution featuring broadband access with fiber being installed at home premises.

From the different access technologies currently available (time-division multipleaccess (TDMA) and wavelength-division multiple-access (WDMA)), optical codedivision multiple-access (OCDMA) stands out due to its inherent characteristics such as architecture flexibility, differentiated services, dynamic bandwidth allocation and security transmissions at the physical layer. In addition, OCDMA networks comply with the requirements of future optical access networks where flexible bandwidth allocation is key to rationalizing network resources. Besides dealing with the statistical analysis of OCDMA networks, this thesis experimentally verifies for the first time the generation of deterministic chaotic dynamics in a commercial VCSEL. It also explores its potential for converging towards a unique solution for future flexible highly-secure optical networks.

The idea behind the use of deterministic chaos is being able to exploit the dynamic, non-linear and nonperiodic properties of chaotic systems to wisely hide the users' data information transmitted through a network, therefore increasing substantially the level of security at the physical layer. This chapter is organized as follows. Section 1.1 lays out the overview of access technologies. Section 1.2 presents the main concept behind the OCDMA multiplexing technique, its advantages and a brief historical background relative to flexible bandwidth allocation and security vulnerabilities. Section 1.3 deals with security vulnerabilities in OCDMA networks and discusses chaotic communications as a potential solution. Finally, the objectives and organization of the thesis are given in Section 1.4. 


\section{INTRODUCTION}

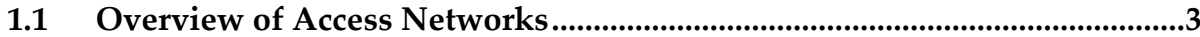

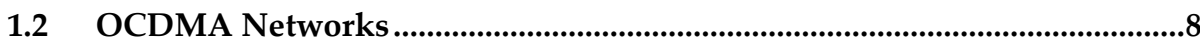

1.3 Towards Chaos Communications …..................................................................15

1.4 Objectives and Organization of the Thesis.................................................18 


\subsection{Overview of Access Networks}

The innate necessity of exchanging and sharing information has motivated individuals to explore the most diverse possibilities and methods to achieve these goals. Amongst several examples of communication trials, a successful case was related in 1876 when Alexander G. Bell and Thomas A. Watson realized the first practical telephone call over a two-mile wire link between Boston and Cambridge. Since that pioneering phone call, and after decades of continuous research and investments, the current state-of-the-art in telecommunications has brought us smart cell phones, optical fiber links, internet, and so on leading to unprecedented volumes of data information traffic.

Several different technologies have been developed to support the traffic demand required by today's highly sophisticated society. Amongst these technologies, two of the most widely deployed solutions are based on asymmetric digital subscriber line (ADSL) and community antenna television (CATV) technology, which predominated in the 2000's and are still available nowadays, depending on the country or region. ADSL and CATV technologies offer broadband access services to end-users using conventional telephone lines and coaxial cables, respectively.

Basically, ADSL has an asymmetric transmission rate between the downstream and upstream channels, in which the uplink has a considerably lower transmission rate. Some of ADSL shortcomings are its limited distance range and capacity in terms of users and bandwidth. CATV, by its turn, was considered an alternative to offer broadband Internet services to end-users in access networks. As with ADSL technology, CATV has limited bandwidth support and asymmetric transmission in uplink and downlink channels, which limits the upstream data traffic of users, in addition to the coaxial cable's limitations such as distance, losses, and heavy layout design. Although ADSL and CATV can both provide certain level of broadband service, these technologies are not suitable to meet the stringent requirements of today's access networks, especially regarding the ever-increasing end-user demand for broadband services. The tremendous bandwidth demand has led access networks, also referred to as last mile, to become the bottleneck of communication systems when compared to the data traffic of other types of networks.

Fiber-optic technology, which conveys information from one point to another using light, is considered the best alternative for next generation broadband access networks. Optical fibers have become the preferred choice of transmission medium, regardless of the type, topology and scale of the network. In addition to their attenuation loss (as low as $0.2 \mathrm{~dB} / \mathrm{km}$ ), optical fibers also have many notable features like, for example, immunity to electromagnetic interference, large transmission bandwidth and low cost, to name but a few [1]. The continuous advances in fiber- 
optic technology have led to a wide variety of economically feasible optical networks capable of transmitting a vast amount of data traffic, allowing new services and technologies to be delivered to the customers. The next wave of innovation will certainly generate numerous opportunities provided by the widespread popularity of multimedia applications such as telemedicine, 3-D TV and interactive e-learning on top of the traditional Internet services. New business models of services will spread the popularity of these applications even further and make them part of everyday life, where people will have convenient and fast access to new emerging multimedia-rich network applications such as interactive HDTV, serious gaming and real time 3-D content generation, where they will be able to project themselves as characters in a tangible environment [2]. Furthermore, the next wave of innovation will generate numerous new opportunities for emerging technology solutions based on tactile Internet [2]. Tactile Internet can handle haptic interaction with visual feedback allowing robotic systems to be controlled remotely with precise human-tomachine interaction. To do so, it requires some technical aspects (from security to flexible systems architecture) not found in current access networks technology. Indeed, current network infrastructures are technically insufficient to support the envisioned demand of upcoming multimedia applications based on tactile Internet [2]. Hence, innovative solutions are needed to lead towards a robust, higher capacity and flexible network capable of providing more efficient use of the available spectrum bandwidth. With that in mind, network resources can be appropriately employed not only to support the popularity of multimedia applications and new business models, but also to cope with the amount of data traffic transmission which has been doubling every two years [3].

The deployment of broadband access networks that brings optical fibers near or very close to the customer premises, known as fiber-to-the-x (FTTx), might be eventually part of this solution [4], [5]. FTTx is a generic term used to describe network architectures of high performance transmission based on optical fiber technology. FTTx solution-based variants are fiber-to-the-building (FTTB), where the final optical fiber equipment is installed in a building; fiber-to-the-curb (FTTC), where the optical fiber cable is directly installed in a street cabinet normally near homes or business places; and finally, FTTH, where the optical fiber is installed at the home premises, such as on the outside wall of a home. Currently, FTTx encompasses the full set of access network architectures that are enabling telecom companies to bring broadband services to the end-user premises. FTTx as an optical access network solution allows more operational efficiency when compared to other access technologies, mainly due to its reduced maintenance and low operational costs.

Generally, optical access networks can be classified into two categories depending on the components involved in its technology, namely [6]: active optical network (AON) and passive optical network (PON). The former employs active components that require electrical power to operate like amplifiers and switches. In contrast, the latter, 
employs only passive components in the signal's path from source to destination, like passive splitters and couplers. PON can reduce the failure probability of lines and improve the reliability of systems. Thus, PON is probably the most attractive technology for access networks, being a promising cost-effective solution to deliver a vast amount of data to end-users [7].

At least three different architectures can be used for practical implementation of optical access networks, namely point-to-point, active star and passive star as illustrated in Figure 1.1. In the former, an exclusive optical fiber is implemented between the central office ( $\mathrm{CO}$ ) premise and every end-user. One fiber channel is allocated to every user as depicted in see Figure 1.1a, which increases considerably the installation and maintenance costs. Moreover, the network architecture of the CO requires a terminal for each user, which increases scale and power problems. In the active star architecture, an active node is employed, and only a single optical feeder fiber is placed between the $\mathrm{CO}$ and active node. Then, an optical fiber is used to connect each user to the active node, see Figure 1.1c. Despite the reduction of the number of fibers used, the active component employed in this architecture raises the power consumption, resulting in a non-efficient solution. A passive node, in turn, is employed in the passive star architecture, resolving both the power consumption and large quantity of optical fiber issues. In this solution, the passive node acts as a power splitter and combiner, receiving signal from the feeder fiber among with the aggregate fibers from the users, see Figure 1.1b. This elegant and efficient solution for access networks considerably reduces the installation and maintenance costs and has become the dominant architecture for practical implementation of FTTH [8].

Existing FTTH solutions need to evolve from current standardized technologies mostly based on time-division multiple-access (TDMA) towards new innovative solutions. While current technologies are based on TDMA, substantial efforts are being made to develop novel solutions for future optical access networks [8], [9]. In networks employing TDMA, each user transmits sequentially and in its own time interval, where an exclusive time slot is assigned to each user. The main disadvantage of this technique, classified as deterministic, is the fact that dedicated pre-assigned time slots do not allow for statistical multiplexing gain, which is a desirable feature in the occurrence of bursty data traffic. Hence, TDMA has an intrinsic limitation on supporting bursty traffic and multirate transmissions, in addition to its strict requirement and additional complexity of end-to-end synchronization along the communication path between two entities [10]. Although TDMA can transparently coexist with currently deployed PON technologies [11], even from a very optimistic point of view, it is not adequate to cope with the requirements of next generation access networks, like capacity, flexible bandwidth allocation and security, and therefore other solutions should be considered [6]. 

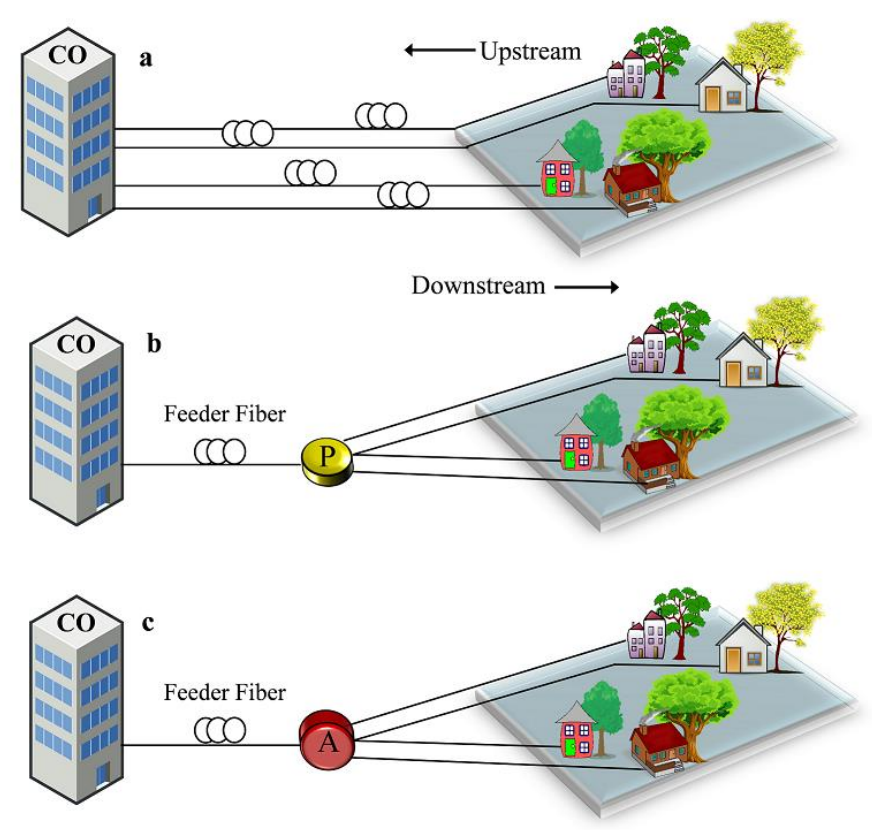

Figure 1.1. Schematic representation of possible FTTH solutions for three different scenarios. a) a point-to-point network with $U$ optical fibers and $2 U$ transceivers. b) a passive optical network with only one feeder fiber and $U$ transceivers featuring the most efficient solution. c) a switched network with one feeder fiber and $2 U+2$ transceivers. CO: central office. P: passive. A: active. Each house represents a user $\in\{1,2, \ldots, U\}$. Picture adapted from [6].

The alternative technologies to support these requirements also include wavelengthdivision multiple-access (WDMA) [5], [12], orthogonal frequency-division multipleaccess (OFDMA) and particularly optical code-division multiplse-access (OCDMA) [1], [13]-[16]. Figure 1.2 depicts schematically the working principle of these multiplexing techniques.

An access network using WDMA can support a virtual point-to-point topology without the concerns associated with multiple users sharing a single downlink transmission channel. All users in WDMA transmit at the same time interval, but in a dedicated assigned wavelength range, as shown in Figure 1.2a. Thus, each channel occupies a narrow optical bandwidth around a central wavelength. The main drawback of WDMA technology is the need of fine tuning and constant monitoring of wavelengths emitted from each source, as well as monitoring of the central wavelength of each filter to eliminate crosstalk among the distinct wavelengths. Furthermore, the smallest bandwidth granularity in this technique is a WDMA window, which might be inadequately coarse or wider than required [6] ,[17], [18]. 


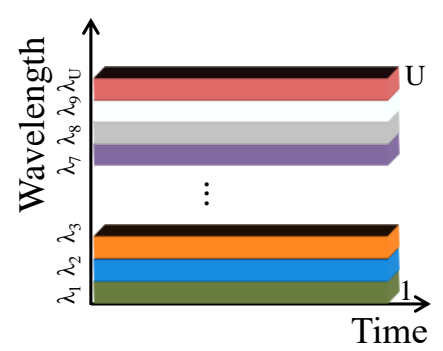

a

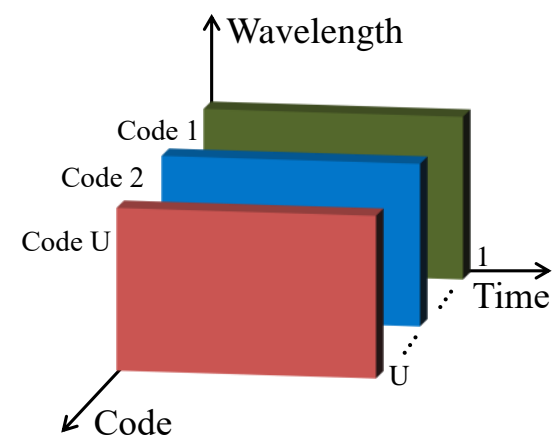

b

Figure 1.2. Multiple access schemes. $U$ is the total number of users, $\lambda_{1}$ is the wavelength $\# 1$, and code 1 is the code sequence \#1. a) WDMA (the colors distinguish the wavelengths), each user transmits in a range of wavelength simultaneously, and b) OCDMA (the colors distinguish the codes), each user transmits simultaneously using its own code sequence, occupying all frequency range available. Figure adapted from [13].

The necessity of a full wavelength allocation even when the traffic is not sufficient to fill the entire capacity, is an inefficient way of resource allocation with wavelength granularity leading to an inefficient utilization of network resources, especially under the requirements of high data rate transmissions and bursty traffic. Hence, innovative solutions for access networks should consider flexible bandwidth allocation, in which the required spectral resources for a transmission is assigned based on the user's traffic demand so that the network resources are used adequately, especially because the physical capacity of conventional optical fibers is not infinite. The awareness of efficiency and the ability to assign network resources in a conscious manner requires innovative solutions capable of providing flexibility in bandwidth allocation. Thus, this multiplexing technique by itself is not the most viable technology for the deployment of future optical access networks, not to mention the hardware and additional protocols imposed by TDMA and WDMA technologies that render the network more complex and costly to operate. The optical CDMA, or simply OCDMA, in contrast, has greater flexibility in bandwidth allocation in addition to being a statistical multiplexing technique with support to multirate transmissions at the physical layer. OCDMA networks can efficiently carry out the bandwidth allocation in a way that network resources are flexibly and consciously allocated based on the user's traffic demand.

In OCDMA-based networks [1], [16] all users transmit at the same time interval and over the same available wavelength range, and for each of these users an exclusive code sequence is assigned, as illustrated in Figure 1.2b. In other words, the network resources are shared among all users to whom unique codes are assigned instead of a time slot like TDMA or a wavelength like WDMA. Thus, all users can access the 
network resources at the same time and using the same wavelength range. Moreover, besides supporting multiple rates and different classes of services at the physical layer, OCDMA is also well suited for bursty data traffic networks. Furthermore, it overcomes the coarse granularity issue of WDMA in an efficient manner reducing it to a subwavelength, i.e., the bandwidth of a single wavelength is shared among many code sequences resulting in several communication channels.

It is worth observing at this point that optical systems based on TDMA and WDMA have fixed channel allocation, which characterize them as deterministic systems. Conversely, optical systems based on OCDMA are characterized as statistical systems since the degradation factor depends on the interfering users present in the system. In other words, the probability of error depends directly on the number of simultaneous users.

Finally, in TDMA and WDMA, time and frequency slots respectively are fixed, hence the capacity in terms of number of simultaneous users is limited. The invariant number of time slots or frequency bands that can be allocated in TDMA and WDMA networks, respectively, mostly remain underused due to the bursty nature of data traffic in access networks. In contrast, in OCDMA networks, there is no strict limit on the number of users that can be supported, only a practical limit ruled by the desired bit error rate (BER), since the signal to interference ratio (SIR) varies inversely with the number of users. Therefore, OCDMA can be considered an efficient FTTH solution suitable to access networks, where a large number of users generating bursty traffic at irregular intervals can be served on the top of its capacity supporting flexible bandwidth allocation and asynchronous transmissions. In contrast to TDMA and WDMA networks with underutilized network resources, OCDMA can allocate the bandwidth flexibly and implement the bandwidth assignment with different granularities, allowing the network bandwidth to be effectively used.

\subsection{OCDMA Networks}

The commercialization of CDMA radio networks for mobile systems all over the world in the 1980's was an extraordinary event in the telecommunications industry. One of the keys to its success was the solution of the power control issue, solved with the advent of digital signal processing. In its infancy, in the early 1970's, CDMA radio technology was mainly considered for military purposes, in which a spread spectrum technique could increase the security of data transmission. In fact, spread spectrum systems have been used in military communications to resist intended interference (jamming) and provide low probability of detection. This multiple access technique allows several users to send data simultaneously over a single communication 
channel by means of assigned code sequences, where the users' data go through an encoding process before being transmitted. The codes allow several users to share the same range of available frequencies during transmissions at any time.

Prucnal et al. proposed in 1986 a very similar encoding process, but now in the optical domain, more specifically, in an optical fiber channel. This encoding technique was introduced as a new channel access method called optical CDMA or OCDMA [19]. Shortly after that, Salehi et al. experimentally demonstrated in 1988 the viability and benefits of encoding a femto-second pulse into several pseudo-random pulses before sending it over an optical fiber channel [20]. Both experimental demonstrations and analysis [19], [20] have enabled a fundamental understanding of the potential advantages offered by the encoding process in the optical domain. Accordingly, those studies triggered a revolution on the way all-optical network processing was viewed, contributing to the establishment of OCDMA networks in 1989 [21]-[23]. Since then, several research groups have contributed to the development of OCDMA techniques and their variants, covering a vast range of topics from interference mitigation [24] and polymeric encoder and decoder modules [25], until 3-D coding schemes [26].

In addition, OCDMA applications have been proposed to many initially unforeseen network scenarios, from avionics solutions [27] to underwater wireless broadband links in oceans [28]. OCDMA is still considered nowadays as a potential candidate to address the problems and requirements faced by current network technologies. This promising multiplexing technique cannot only potentially enable telecom service providers to cost effectively cope with the capacity constraints of their existing optical network solutions, it can also satisfy main key requirements of next generation access networks, such as dynamical allocation of bandwidth [8], [10].

OCDMA technology has emerged as an attractive architecture in flexible, robust and high-capacity passive optical networks that straightforwardly provides differentiated services, dynamic bandwidth allocation and security transmissions at the physical layer. The technology roadmap, also the recent progress and maturity of optical components [8], [29]-[37] strongly indicate that OCDMA tends to follow the legacy of CDMA radio networks [38].

Furthermore, OCDMA technology can potentially fulfil the current demand and principal requirements faced by today's network solutions, which makes it more suitable for next generation optical access networks. For example, among the many advantages provided by this technology the following benefits stand out [1]: i) users can asynchronously access and transmit in the network, which is a key issue for practical network deployment; ii) users can embark or exit without any supervision; iii) bursty traffic and statistical multiplexing is supported; iv) secure data transmission capability using a pseudo-random code at the physical layer; v) high 
compatibility with WDM technology; and vi) incoherent receptors based on intensitymodulation direct-detection (IM-DD) scheme.

OCDMA, as initially mentioned, can be classified as a spread spectrum technique, since it increases the physical bandwidth of a channel by employing spreading codes. In general, the basic principle of spread spectrum concerns any communication system where the bandwidth of a signal transmitted over the channel is wider than the bandwidth required to transmit the original information signal. To put it simply, it extends the original data bandwidth over a broad bandwidth via a spreading code sequence. In this type of system, the baseband signal bandwidth is intentionally spread over a wider bandwidth as illustrated in Figure 1.3. Amongst many possibilities, the spreading can be carried out via direct-sequence (DS), where data bits are directly multiplied by a code sequence and thereby divided into pulses known as chips, or can be carried out via frequency-hopping $(\mathrm{FH})$ techniques, where data bits are spread over several frequency bands.

Furthermore, OCDMA networks can be further divided into two broad categories depending on the way a given user's code is applied into the optical signal [6], namely, incoherent and coherent. In incoherent techniques, a unipolar code $(0,1)$ such as optical orthogonal code (OOC) or fast frequency hopping (FFH)-based code is used along with on-off keying $(\mathrm{OOK})$ modulation format, resulting in an encoding process based on optical intensity. In coherent techniques, a bipolar code $(-1,+1)$ such as Gold code or Hadamard code is used, which results in an encoding process based on the signal phase. Nevertheless, most of the employed encoding techniques are characterized by the manipulation of the signal intensity along with OOK, due to both its simplicity and IM-DD nature. For example, in OOK, the data information bit " 1 " is represented by the presence of an optical pulse, whereas the data bit " 0 " is represented by the absence of an optical pulse.

Amplitude

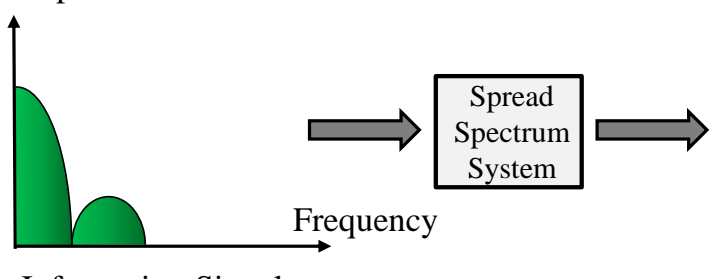

Information Signal
Amplitude

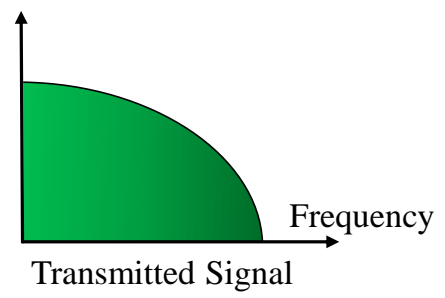

Figure 1.3. Bandwidth required to represent the information signal and bandwidth utilized by the spread spectrum system for the transmission. 
In regards to the transmission method, OCDMA networks can be widely defined in synchronous and asynchronous depending on the employed technology. Though each one of them has its own benefits, asynchronous networks stand out due to several reasons such as simplified network infrastructure with no need of synchronization between transmitters and receivers, potential low-cost with less hardware required, simpler maintenance and insertion of new coming users, and greater scalability as well as flexibility. One of the most striking features of OCDMA technology is that the users' data information is encoded before being sent to the transmitting channel. Throughout the encoding process, each user's data bit is divided into time slots denoted as chips, where a specific sequence of these chips establishes a code. The code length defines the total number of chips in each code and the code weight defines the total number of pulses in each code. Normally, all code sequences employed in a conventional OCDMA network are based on equal code weights and lengths with the assumption of supporting equal data rates and equal error rate (BER) for all users. In contrast, new codes with different weights and lengths have been developed to provide multiservice (or quality of service (QoS)) and multirate transmissions, where users assigned with high-weight and short-length codes transmit at higher data rates with higher QoS (or equivalently lower levels of BER). Conversely, classes of users assigned with low-weight and long-length codes transmit at lower data rates with lower QoS (higher levels of BER).

An OCDMA network can efficiently carry out bandwidth allocation based on a user's traffic demand by means of different code lengths rendering this network as flexible. Flexible networks can divide their users into groups or classes, where each class has its own transmission specifications (data rate and QoS) [39]. Figure 1.4 illustrates a flexible OCDMA network with users divided by classes, where each user class has a different transmission rate and QoS. In this example, all users from class \#1 employ short-code length (high-data rates), whereas users from class \#j employ long-code length (low-data rates).

Regardless of the network category, the main deleterious noise source in single-rate and multirate OCDMA networks is known as multiple-access interference (MAI). MAI can be briefly defined as the crosstalk among many different users' signals that share a common communication channel simultaneously. MAI occurs whenever several users coexist simultaneously in a channel so that signals of interfering users generate noise, i.e., make interference on the desired user's signal. Moreover, MAI is directly proportional to the number of simultaneous users on the network in a way that the higher the number of users, the higher the level of MAI generated and, consequently, the higher the desired signal's degradation. Despite the existence of other sources of noise in OCDMA networks, MAI is normally treated as the dominant one in most of the cases [40]-[44]. 


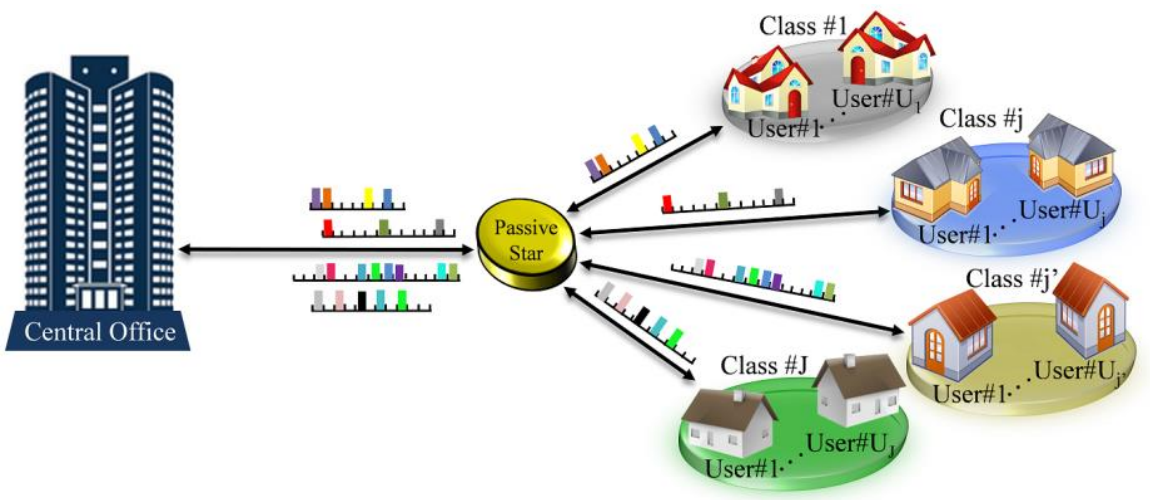

Figure 1.4. Viable architecture of a flexible OCDMA network with support to dynamic bandwidth allocation and multiservice transmissions. Each user class has its own code parameters specification. Each user has a unique code. In this example, link direction, network broadcast and packet switching concept are not illustrated for the sake of simplicity. Picture taken from [39].

Normally, MAI increases the transmitted power of the desired user's signal at the receiver side, which might occasionally change a transmitted data bit from " 0 " to " 1 ", resulting in a considerably increase of the bit error rate (BER), hence limiting the overall network performance. Even though the probability density function (PDF) of the MAI follows a binomial distribution, it is usually approximated by a gaussian distribution [43], [45]-[47]. A common argument used to justify this approximation is that should the number of simultaneous users in a network become large enough MAI would also become large, resulting in an acceptable approximation. This argument, widely adopted in the literature [40], [48]-[50], is based on the central limit theorem (CLT), which establishes how a PDF converges towards the gaussian distribution, even if the original variable itself is not normally distributed. However, as pointed out in [46], the gaussian approximation statistics can be highly questionable for BER estimation in OCDMA networks, even for a network scenario accommodating several users. Therefore, the gaussian distribution might not provide accurate results for performance evaluation of flexible OCDMA networks, regardless of the code family used.

Several works have been dedicated to developing codes for OCDMA networks since its early progress. Traditional encoding schemes are based in time or frequency domain or a combination of both. When only one domain is adopted, the code is classified as one-dimensional (1-D), and when a combination is used for encoding, two-dimensional (2-D) codes are formed. The first originally proposed 1-D OOC [21] [23] was based on equal code weights and code lengths with the assumption of providing equal data rate, i.e., single rate transmission and equal error rate, resulting in single service transmission. However, the first multirate OCDMA approach was based on 1-D OOC and was limited to two rates only [45]. 
Recently, 1-D OOC with arbitrary code weights and different code lengths was proposed aiming at multiple rates and multiple-QoS (or equivalently, multiple-BER) transmissions. This $1-\mathrm{D}$ OOC is referred to as multi-weight, multi-length OOC (MWML-OOC) [42] in which distinct weights among the codes provide different levels of service or BER (multi-QoS), while codes with distinct lengths provide different data rate transmissions. Moreover, a MWML-OOC code set has out-ofphase autocorrelation and cross-correlation bounded by one at the most, which defines it as codes with good correlation properties.

The quantity of available OOC sequences (and most of its variants) is limited by the well-known Johnson bound, which defines the total number of available codes [6]. Since this number of available codes is a function of the code parameters such as the correlation properties, 1-D MWML-OOC with correlation values bound by one at maximum generally presents low number of available codes, meaning that 1 -D codes may have low cardinality. An alternative to circumvent this drawback is to manipulate both time and frequency domains simultaneously by means of 2-D codes. Two dimensional codes are of particular interest since they allow considerable reduction in the temporal dimension of code sequences in relation to 1-D codes, without hindering the code cardinality or network's performance. Also, they more easily satisfy the required code correlation properties [51]-[53].

Accordingly, it has been proposed the addition of the wavelength dimension to the code design to construct codes with larger cardinality while maintaining good autoand cross-correlation properties. In a 2-D coding scheme, the spreading of chips is carried out in time and wavelength, simultaneously. In this case, the time and wavelength selections are established by a construction algorithm, which increases the flexibility of code design. Many 2-D codes have been proposed in the literature to support simultaneously multiservice and multirate transmissions.

A distinct and worth investigating code, denominated 2-D multi-weight multi-code OOC (2-D MWML-OOC), has been recently proposed in [54]. This code has good correlation properties and large cardinality, and presents a reasonably simple construction algorithm. Furthermore, in this 2-D MWML-OOC, the number of wavelengths and code-length and -weight can be chosen independently, which allows arbitrary data rates and QoS differentiation to be achieved. Since 2-D codes normally have short length, they present advantages such as low chip rate and network latency, and high spectral efficiency [54].

Another attractive 2-D code scheme, that also performs in time and wavelength domains simultaneously, is characterized by frequency hopping $(\mathrm{FH})$. In frequency hopping, the carrier frequency of the transmitter hops in accordance with an apparently random pattern. This pattern is in fact a pseudo-random code sequence. When a hop occurs in a time slot smaller than the information signal period, the 
process is defined as fast frequency hopping (FFH), in which fast jumps occur at wavelengths that change for every pulse of a given code. The optical FFH (or OFFH) is a promising 2-D code to be implemented in flexible OCDMA networks. The OFFHCDMA network was originally proposed by Fathallah [40] and is based on a series of multiple Bragg gratings (MBGs) to generate fast hopping frequency patterns. These gratings spectrally and temporally slice an incoming broadband pulse into several components, hence generating optical frequency patterns. The passive all-optical coding based on MBGs allows both low-cost and robust implementation of OFFHCDMA networks [55]. An inherent characteristic of OFFH systems is that the frequency changes at a significantly higher rate than the information rate, which means that each pulse in a code sequence is transmitted at an exclusive wavelength. Thus, OFFH systems are defined by several frequency hops within each data bit. This technology was originally proposed for single rate transmissions and employing equal code lengths to guarantee equal data rate for all users [40].

Inaty et al. [47], [56], in contrast, have proposed an OFFH-CDMA (or FFH-OCDMA) network with support to multirate transmissions. This network provides multiple rates through different code lengths while keeping constant the chip period. To this end, encoders and decoders based on MBGs of different lengths were suggested, i.e., different number of Bragg gratings. Another possibility to vary the number of gratings is to tune out the wavelengths from longer codes so that they are not reflected into the working bandwidth of the network. In addition, each Bragg grating can also be individually tuned to adjust an intended wavelength from an available bandwidth range [55]. The wavelengths tuned out of the working bandwidth are no longer reflected by the Bragg gratings of the encoder/decoder [47], i.e., the grating becomes transparent to these wavelengths. This is very attractive for reconfiguring the encoder/decoder [55]. Accordingly, this thesis also deals with 2-D encoding schemes based on FFH as a robust way for obtaining multi length codes for flexible OCDMA networks with support to multirate transmissions.

Independently of the technique adopted to support multirate transmissions, network designers are interested in selecting the most effective architecture or design constraints a system can support so that they satisfy the requirements for the expected performance. A useful figure of merit to perform such a measurement is known as the packet throughput. Although the BER is a good figure of merit to evaluate the network performance, the packet throughput also has its advantages, being considered an important metric of performance evaluation as it provides the rate of packets transmitted with success in a network. For example, it denotes how steady (the rate of successful of) the data information delivery is over a communication channel. Accordingly, the throughput is a good indicator of the performance status of a network and useful for system troubleshooting. Hence, in contrast to the mathematical formalisms developed for single-rate networks, in this thesis it is of 
general interest to consider and investigate the packet throughput performance of flexible OCDMA networks.

In this context, several works have addressed the packet throughput performance of single rate networks [53], [57]-[63]. Nevertheless, to the best of our knowledge, the development of a mathematical formalism to accurately investigate the packet throughput performance of flexible OCDMA networks with support to multirate and QoS transmissions is a missing point in the literature and will be addressed in this thesis.

\subsection{Towards Chaos Communications}

Most networks' security schemes rely on encryption methods to provide security data transmissions against potential eavesdroppers. These methods are normally implemented at higher protocol layers and might be inefficient in terms of dependability, robustness, and availability. Threats are becoming more sophisticated and targeted than ever before and novel solutions should exploit the potential of physical networking so that they can provide safe connections and privacy from the physical layer.

The intrinsic use of encoding schemes grants OCDMA networks a higher level of security when compared to other non-encoding technologies like, for example, TDMA, WDMA and OFDMA [64]. In fact, the integrity and security of users' information are ensured by means of unique signature codes. In OCDMA networks, multiple users share the same communication medium via exclusive assigned codes and usually realize transmissions employing a widely-adopted modulation format, the so-called OOK, which manipulates the intensity of an optical signal. The simplicity of OOK implementation has always been the main reason for its use in communication systems [65]. In the past, it was necessary for a modulation technique to maintain a commitment with simplicity in detection. Accordingly, OOK modulation is conducive to optical access networks and used until nowadays, even for high rate transmissions.

However, intensity-modulated OOK signals might present certain vulnerabilities and risks depending on the network scenario considered, as identified and reported in [66]. Accordingly, an OOK-based encoded signal can be easily detected and have its bit information retrieved simply by power detection [66], regardless of the encoding scheme used, when the network has only a single user transmitting. The encoded signal can be detected by an illegal eavesdropping device for example using a photodetector, without knowledge of the used code. Again, it can only happen in a 
network with one single user transmitting OOK-based signals. Even though access networks are supposed to serve several users, this network scenario is often the situation of upstream traffic transmissions in a star network architecture, where an end-user does not share the access optical up-link and realizes transmissions alone. Despite the proposal of some partial solutions to reduce security vulnerabilities associated with the use of OOK modulation formats [66]-[68] there are still a few open issues that might expose the users' information transmitted over a network, especially in the upstream direction. Novel solutions providing higher security levels at the physical layer should be further considered since they have become an indispensable requirement of next generation access networks.

A possible and straightforward alternative to circumvent security issues in OCDMA networks is to consider the deployment of advanced modulation formats. A large variety of optical devices has become available for applications based on access networks with the remarkable progress in optical technology observed over the last years. Among them, encoders and decoders, source- (without a laser source) and color-less (non-wavelength specific), in-line dispersion-compensation-free using code bandwidth optimization, and thresholding devices [8]. Advanced modulation formats based on phase-shift keying (PSK) have also gained considerable attention. For example, PSK modulation, such as binary PSK (BPSK) and quadrature PSK (QPSK), have been implemented using all-optical modulation format conversion techniques (OOK to BPSK, and BPSK to QPSK) [69].

Furthermore, PSK-based OCDMA networks supporting high level of security as well as data rate transmissions have been suggested recently [65]. The PSK-based modulation formats represent the information bits by changing the phase of the optical field. Any number of phases might be used in a modulated PSK signal, but the higher the PSK constellation deployed the more complex it gets. Although several researches perceive PSK techniques as potential candidates for symbol modulation in optical access networks, their massive deployment is still not viable at this time due to the high costs involved in these complex modulation techniques. Therefore, it can be concluded that security issues in optical access networks still remain open to be exploited, especially if one is looking for a robust and cost-efficient solution for next generation access networks, where the requirement of higher security levels implemented at the physical layer has become crucial.

However, OCDMA with several users still offers a certain level of security and builtin privacy because a given receiver can at first only retrieve the transmitted data with the knowledge of the employed code. This is true since the original signal is spread using a pseudo-random code, in which the code makes the signal to appear randomlike and more resistant against intended interference. The OCDMA encoding concept is very similar to other communication techniques also based on spread-spectrum and known as deterministic chaos. The term chaos is normally used to describe 
irregular fluctuations that are governed by deterministic rules and can be described by using a set of equations without any stochastic terms.

Basically, in optical chaos networks, the data information of a user is modulated with a chaotic carrier and then transmitted over an optical channel. At the receiver side, the original information is retrieved via a synchronization technique, used to reproduce a nearly identical chaotic carrier as used in the transmitter. Accordingly, once synchronized, the data information can be retrieved by subtracting the synchronized system from the transmitted signal. Normally, synchronization in chaotic networks takes place when the trajectories of two systems converge towards the same value and remain evolving equally in time with respect to each other.

Chaos communications provides additional levels of security at the physical layer when compared to traditional communication schemes. In a chaos-based network, the nonlinear and non-periodic features of chaotic signals make them more robust against interference and jamming. Owing to the unpredictable behavior of chaotic signals, the data information can be efficiently hidden in chaotic signals at the physical network level to be transmitted. Deterministic chaos aiming at increasing the level of security has been considered as a potential communication solution for highly-secure and robust optical access networks since its prediction by eventual eavesdroppers is not a trivial task to be accomplished. Furthermore, chaos systems have also been suggested as possible solutions not only to improve the security level of optical networks, but also to enhance the capacity of CDMA radio networks [70], [71], which can be potentially extended also to OCDMA networks.

One of the most well-known examples of chaotic systems is based on semiconductor lasers with optical feedback, where a small fraction of the laser output beam is reflected from an external mirror and fed back into the laser cavity. Then, the regular optical intensity in the laser cavity is perturbed by the self-feedback light generating complex chaotic dynamics in the laser output. In fact, chaos communications have been proposed under many different schemes, in which most of them relies somehow on an optical source of chaos generation based on an external mechanism such as optical feedback as previously mentioned or current injection. For example, chaotic sources based on time-delay optical feedback add complexity to the system as well as cost, reduce portability, and limit their applicability since it becomes complex to deal with and scale-up a single system comprising hundreds of individual chaotic sources.

In this context, the problem of security vulnerabilities in optical networks is tackled here from a distinct perspective, in which we focus on a new cost-effective solution based on chaos communications that can be useful not only for OCDMA networks, but also for optical communication systems in general. Hence, we propose here a sufficiently robust and low-cost solution for optical chaos generation based on 
deterministic chaos that can be used to increase the level of security in general optical networks.

\subsection{Objectives and Organization of the Thesis}

The arduous work during recent years on technological solutions for future optical access networks have tried to cope with the requirements of emerging communication systems. However, in due course it became apparent that several aspects are still unsolved as pointed out in previous discussions on flexible and highly-secure access network, in which capabilities of providing dynamical bandwidth allocation and higher security at the physical layer must be considered. In this way, this doctoral thesis contributes to two major study areas: flexible allocation of network resources and cost-efficient chaos generation for security purposes. Henceforth, the original contributions of this thesis are briefly outlined as follows:

I. New mathematical formalisms for bit error rate, packet throughput and packet delay to assess the performance of flexible OCDMA networks with support to multiservice multirate transmissions. The proposed analytical formalisms do not require the knowledge a priori of the users' code sequences, which means that the network performance can be addressed in a simple and straightforward manner using the code parameters only. In addition, the developed analytical formalisms account for a general number of distinct users' classes as well as general probability of interference among users. Hence, these formalisms can be successfully applied for performance evaluation of flexible OCDMA networks not only under any number of users' classes present in the network, but also for most spreading codes with good correlation properties. Finally, the formalisms allow the performance evaluation of flexible networks when only the data bit " 0 " is transmitted or when both data bits " 0 " and " 1 " are sent to transmission. This offers more flexibility to choose design constraints so that a network can satisfy the requirements of an expected performance.

II. A new mathematical formalism for BER and packet throughput performance evaluation of OCDMA networks with support to multirate transmissions via assignment of several codes for each single user, the so-called multicode technique. 
III. A new architecture of IP traffic transmission over flexible OCDMA networks with support to multiservice multirate transmissions, in which the proposed network architecture is independent of the spreading code employed and does not require any new optical processing technology.

IV. A new cost-efficient, robust and practical optical chaos generator based on a commercial quantum-well vertical-cavity surface-emitting laser (QWVCSEL) operating in a free-running configuration. For a potential widespread use, this new solution has been considered as simple and robust as possible, in which an easily replicable holder loads mechanical stress onto an off-the-shelf QW-VCSEL and successfully triggers chaotic polarization dynamics. This behaviour has been achieved, for the first time, without the additional complexity of optical feedback or any other external forcing. This paramount solution, based on commercial devices, paves the way not only towards new security platforms in optical access networks, but also for a large variety of chaos-based solutions and emerging applications such as random bit generation, cryptography and quantum networks

This thesis is organized as follows:

Chapter 2 deals with techniques used to encode the data bit information of users before being sent to transmission. Encoding techniques suitable for flexible OCDMA networks based on 1-D and 2-D codes are addressed with emphasis on their design based on different weights and lengths exclusively for providing multiservice, multirate transmissions. In addition, the 1-D and 2-D passive encoding processes that use optical delay lines and/or wavelength-division multiplexing (WDM) multiplexers as well as demultiplexers are described. This chapter also addresses 2-D encoding schemes for the generation of fast frequency hopping codes based on fiber Brag gratings. This all-optical passive scheme is an efficient way of generating incoherent codes. One of the most important degrading factors in OCDMA networks, known as MAI, is addressed so that one can better understand the impact of this intrinsic network performance limitator. Then, the last part of the chapter regards two different techniques normally employed to achieve multirate transmissions and suggests possible ways to implement them.

Chapter 3, by its turn, introduces the mathematical formalisms and analytical models developed and used here to evaluate the performance of flexible OCDMA networks capable of providing multiservice multirate transmissions. Then, new expressions for the BER, packet throughput and packet delay are derived. Moreover, new expressions for evaluating the PER and packet throughput of a network architecture of IP traffic transmission over flexible OCDMA are also derived. Next, new BER and 


\section{INTRODUCTION}

packet throughput expressions for the evaluation performance of OCDMA networks with support to multirate transmissions via the multicode technique are presented.

Chapter 4 presents the numerical results obtained with the proposed analytical formalisms, in which the performance of several network scenarios is assessed. Validations of the proposed formalisms are also carried out, where two validation criteria are assumed. Then, the packet throughput performance is compared assuming Poisson, binomial and Markov Chain approaches for the composite packet arrivals.

Chapter 5 deals with an alternative cost efficient-solution to generate optical chaos from an off-the-shelf QW-VCSEL subjected to mechanical stress. We experimentally demonstrate that polarization chaotic dynamics can be obtained in a consistent and easily reproducible way from ordinary commercial QW-VCSELs operating in a freerunning configuration. This is done using a simple, low-cost, easily replicable holder to introduce anisotropic strain in the laser cavity.

Chapter 6 summarizes the original contributions of this doctoral thesis and outlines perspectives for future work. In addition, the impact of this research is discussed as it opens innovative ways to secure communication networks. 


\section{Chapter 2 Flexible OCDMA Networks}

This chapter focuses on encoding techniques employed in conventional and flexible OCDMA networks so that one can understand not only the operational principle of this multiplexing technology, but also how multiservice, multirate transmissions are achieved as well as how interference among the users' signals occur. Hence, this chapter covers an overview of the state-of-the-art describing 1-D and 2-D encoding schemes and multirate techniques prior addressing the novel contributions given in the next chapters. The first originally proposed encoding scheme was based on 1-D, in which the code set had equal code weights and lengths so that they could satisfy the correlation properties and guarantee the same service and transmission rate for all users. Recently, 1-D codes designed with different weights and lengths have been proposed to support multiservice, multirate transmissions. Their generation using optical delay lines is addressed here. This chapter also describes how the 2-D encoding process is carried out with the use of optical delay lines and multiplexers/demultiplexes, and provides the fundamentals for the generation of fast frequency hopping codes based on fiber Brag gratings. Next, the error occurring process for the evaluation and understanding of multiple-access interference (MAI), which is often considered the most important noise source in the analysis of OCDMA networks, is presented. Finally, the chapter ends with a detailed explanation regarding the two most usual multirate techniques found in the literature, referred here as multilength code and multicode, and how they provide multirate transmissions in OCDMA networks.

$2.1 \quad$ Encoding .......................................................................................................22

2.2 1-D Encoding.........................................................................................................23

2.2.1 Encoding in Time Domain, 1-D MWML-OOC ..........................................24

$2.3 \quad$ 2-D Encoding........................................................................................................29

2.3.1 Frequency Hopping, FH-OCDMA ...........................................................

2.3.2 Fast Frequency Hopping, FFH-OCDMA ……………………………….......34

2.4 Multiple Access Interference ............................................................................39

$2.5 \quad$ Multirate Techniques .............................................................................................41 


\subsection{Encoding}

Normally, multiple users share simultaneously the common OCDMA network resources by means of uniquely assigned code sequences, where codes can be spread in time, frequency, space or phase domains, or a combination of them. When only one of these domains is adopted, these codes are classified as one-dimensional (1-D) codes. Likewise, when two or three domains are considered for encoding, twodimensional (2-D) or three-dimensional (3-D) codes are formed, respectively. The latter has a high degree of complexity considering its practical implementations and is not addressed here. Noteworthy, the frequency domain is also referred to as wavelength domain, and both terminologies are used interchangeably in this thesis.

Furthermore, OCDMA can also be classified into two large categories depending on the way a given user's code sequence is applied into the optical signal [6], [72], namely, incoherent and coherent. In incoherent techniques, a unipolar code $(0,1)$ such as MWML-OOC or FFH is used along with on-off keying (OOK) modulation format, resulting in an encoding process based on optical intensity. On the other hand, in coherent techniques, a bipolar code $(-1,+1)$ such as Gold code or Hadamard code is used, which results in an encoding process based on the signal phase. Nevertheless, most of the encoding techniques employed in OCDMA are characterized by the manipulation of the signal intensity via OOK modulation due to both its simplicity and intensity-modulation direct-detection (IM-DD) nature. For example, in OOK, the data information bit " 1 " is represented by the presence of optical pulse, whereas the data bit " 0 " is represented by the absence of optical pulse.

Each user's data bit in OCDMA is divided into time slots denoted as chips, where a specific sequence of these chips establishes a code. The code length defines the total number of chips in each code. By its turn, the code weight defines the total number of pulses (number of illuminated short pulses) in each code. As already mentioned, the users employ their own unique codes every time to transmit a data bit " 1 ", represented by the presence of short optical pulses, whereas nothing is transmitted (absence of short pulses) for data bit " 0 ". At each receiver side, the signals multiplexed and broadcasted through the optical channel are correlated with an already known code to retrieve the desired signal.

In general, the codes assigned to users need to satisfy some correlation properties such as out-of-phase autocorrelation and cross-correlation so that several simultaneous users can be eventually accommodated in a network. Hence, code generator algorithms seek to both maximize the orthogonality (degree of differentiation between codes) and the cardinality (number of maximum possible available codes and, consequently, users). A code design with good correlation 
properties accounts for values of shift autocorrelation and cross-correlation bounded by one at most.

Regardless of the code scheme employed in an OCDMA network, the latter can be considered conventional when only a single transmission rate is available, which means that the bandwidth allocation is fixed for all users. This is probably an inefficient way of assigning network resources, especially when the traffic conditions of access networks are considered such as bursty and/or high-rate transmissions. An elegant and efficient alternative to wisely distribute the network resources is to flexibly change the spreading factor of the encoding scheme based on the users' transmission demand so that the finite network resource can be used more appropriately. In this way, the bandwidth resource is allocated according to the transmission requirements of the users in a flexible manner.

Bear in mind that changing or varying the spreading factor of the network is analogous to changing the bit period which, by its turn, corresponds to changing the code length. Following this reasoning, OCDMA networks with such a flexible capability of allocating bandwidth and consequently code length are classified as flexible networks. Putting it simply, an OCDMA network scheme that can wisely carry out the bandwidth allocation based on the user's traffic demand by means of different code lengths renders this network as flexible. Since the users of a flexible OCDMA network can have different requirements in terms of bandwidth and QoS, they can be organized into different classes, where each class has a different specification. For example, class- 1 users of a J-class network can be classified in a way that they are assigned to high transmission rates and high QoS, while class-2 users are assigned to low transmission rates but high QoS. As a matter of fact, flexible networks usually have their users divided into classes so that each group of users can have its transmission demand satisfied accordingly. The key features of 1-D and 2-D codes that are often employed in OCDMA networks will be presented next.

\subsection{1-D Encoding}

Code generation is certainly at the heart of OCDMA technology. A good choice of encoding scheme impacts positively not only the orthogonality and cardinality of the generated code sequences, but also the overall network performance. Currently, there are several coding schemes available to perform the encoding and decoding of users' data bit in OCDMA. One of the first schemes proposed for this task is known as temporal encoding, which encodes an information bit dividing it into chips and spreading them in time within the original bit period. This scheme is classified as 1D encoding and represents codes such as the 1-D MWML-OOC. This code is usually 
very sparse, which results in large length and small code weight, with values of crosscorrelation as well as nonzero (temporal) shift autocorrelation less than or equal to one to minimize the MAI. By carrying out spreading in one dimension only, 1-D MWML-OOC generally has a low number of available codes, which implies in low cardinality and consequently low number of possible users accommodated in a network. The following subsection describes in detail 1-D encoding schemes employed in time domain.

\subsubsection{Encoding in Time Domain, 1-D MWML-OOC}

In conventional and multirate OCDMA networks, one (not-encoded) data bit corresponds to one pulse with a much shorter duration $\left(T_{c}\right)$ than the data bit of duration $T_{b}$. The codification process divides the bit period into consecutively $L$ (code length) time subintervals of duration $T_{c}$ denominated as chips. In addition, the code weight $W$ defines the number of temporal chips that are lit with optical pulses. Thus, the initial incident pulse, generated before the encoding process, should have a period equal or less than $T_{c}$ so that interferences within a sequence attributed to a given user do not occur. An example that illustrates the previously mentioned concept is shown in Figure 2.1a, where an incident (not-encoded) bit, represented by a pulse of short duration $T_{c}$, is sent to be encoded.

One of the first 1-D encoding schemes for conventional OCDMA was proposed by Salehi et al. [21], [22], [73] which consisted of equal code length and weight for the entire set of generated OOC codes. This entails in the same transmission rate and BER level for all users of the network. However, OOC with arbitrary code weight and different code length, known as MWML-OOC, has been proposed to support multirate and different levels of BER (or equivalently, different QoS levels or multiservice) transmissions [42].

The capability to support multiple services and rates is directly related to the weight and length variation of the codes in the sense that different code weights provide service differentiation (QoS), while different code lengths provide data rate differentiation. Accordingly, high-weight and short-length codes are assigned to high-QoS and high-data rate users, respectively. This straightforward manner of achieving multiple rate transmissions in flexible OCDMA networks, consists of variating the bit duration $\left(T_{b}\right)$ in a way that high-rate users have shorter bit period and low-rate users have larger bit period. This technique is referred here as multilength code and is addressed in detail in Subsection 2.5. Remarkably, varying the code length results in modifying the spreading gain of the network while maintaining the spread bandwidth constant. 


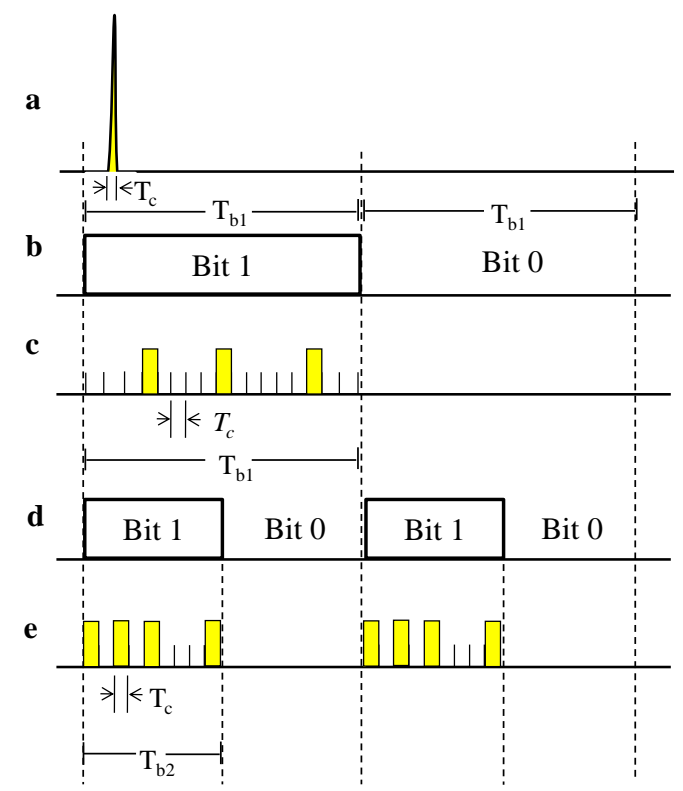

Figure 2.1. Scheme of the encoding process of a user's data bit into an OCDMA code sequence formed by several short pulses. a) Short pulse representing the generated data bit of user class-1. b) Data bit stream of user class-1. c) Encoded short pulses representing the code sequence of class1. d) Data bit stream of user class-2. e) Encoded short pulses representing the code sequence of class-1. Note that $T_{c}$ is constant. For the sake of simplicity, only the incident (not-encoded) generated data bit for class-1 user is shown in (a).

In this fashion, flexible OCDMA networks with support to multirate transmissions via code length variation are classified as such since they dynamically allocate the bandwidth demanded for a certain data transmission. A better insight into the concepts of encoding and multirate techniques is provided with Figure 2.1, which illustrates the transmission of a two-data bit train $\{10\}$ for a user transmitting at low rates and a four-data bit train $\left\{\begin{array}{llll}1 & 0 & 1 & 0\end{array}\right\}$ for a user transmitting at high rates, respectively. Observe that the encoded data bits alternates between " 0 " and " 1 " with the absence or presence of optical pulse, respectively. For the sake of simplicity and space, only the generated information data bit for the low rate user is shown in Figure 2.1a. Notice that bits from the high-rate user are shorter in time $\left(T_{b}\right)$ than bits from the low-rate user, as shown in Figure 2.1b and Figure 2.1d. Moreover, the bit period and code length of the low rate user, Figure 2.1c, is twice as large as that of the high rate user, Figure 2.1e, which implies that the former transmits at half the data rate as that of the high rate user. In this example, the low rate user will most likely get a worse BER level (or QoS) than the high rate user due to the fewer number of pulses (code weight) in the low rate user's code. This is illustrated in Figure 2.1c. It is noteworthy that the chip duration $\left(T_{c}\right)$ is kept constant for all bits and users of the multirate 


\section{FLEXIBLE OCDMA NETWORKS}

flexible network. Even though this figure illustrates an example with two distinct types of users only, the users of a flexible OCDMA network can be divided into several classes, where each class has its own transmission specifications (data rate and QoS). Finally, the example of this encoding scheme suits well the so-called 1-D MWML-OOC, which stands out and is of particular interest due to its good correlation proprieties, possibility of being generated for a general number of classes with design flexibility, and simple practical implementation. A practical implementation of 1-D MWML-OOC employs optical delay lines (ODLs) interconnected to pulse time allocation. The encoding process of data bit " 1 " into a 1D MWML-OOC using ODLs is illustrated in Figure 2.2. In this scheme, the information data bit is represented by an optical pulse, with the duration of each chip related to the code length in a way that $T_{c}=T_{b 2} / L$. The encoder consists of a $1: W$ splitter, $W: 1$ combiner, and W ODLs as depicted in Figure 2.2b. The number of delay lines is equal to the code weight, and the delay of each line is equal to the nonzero position of the code. The system uses OOK modulation and an optical pulse is sent to the encoder whenever the data bit to be transmitted is " 1 " and nothing is sent when the data bit is " 0 ". The optical pulse of the bit " 1 " is encoded by the encoder, whose output is an optical signal that corresponds to an MWML-OOC. Nothing is encoded when the bit " 0 " is sent to the encoder. Initially, a wavelength-independent short pulse with duration $T_{c}$ and high amplitude that corresponds to the data bit " 1 " is sent to the device to be encoded, as shown in Figure 2.2a. The initial pulse is split into $W$ pulses and their respective ODLs generate a temporal delay following a preestablished design to form a code sequence, as shown in Figure 2.2b and Figure 2.2c, respectively. The codification corresponds to the generation of short pulses whose number is proportional to the code weight $(W=4)$. Users from other classes can eventually have a different number of code weight and encoded short pulses.
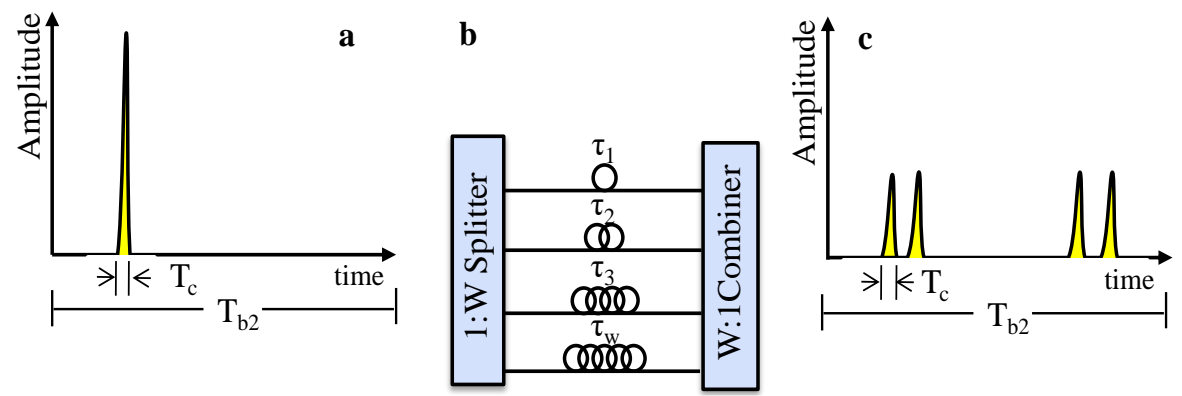

Figure 2.2. 1-D MWML-OOC encoding scheme based on time spreading. a) A wavelengthindependent short pulse corresponding to data bit " 1 " is sent to the encoder. b) Possible encoder scheme based on ODLs, splitter and combiner. c) Encoded 1-D MWML-OOC signal. Picture adapted from [1]. 
Signals from other users, represented by their specific code sequence composed of short pulses are combined via a star coupler (not shown in the figure) and transmitted over the network. At the receiver side, a set of similar ODLs in a configuration complementary to that used in the encoder is employed in the decoder to reconstruct the original pulse [19], received after transmission of the encoded signal over an optical channel as illustrated in Figure 2.3a. This allows for an alignment of the pulses that compose the transmitted encoded signal. The decoder consists of a $1: W$ splitter, $W: 1$ combiner, and $W$ ODLs as depicted in Figure $2.3 \mathrm{~b}$.

The decoding process is carried out by means of intensity correlation in which pulses positioned correctly form a well-defined autocorrelation pattern, otherwise they form a background (cross-correlated) interfering signal. These two possible outputs of the decoding process are shown in Figure 2.3c and Figure 2.3d. The output of the decoder is an auto-correlation peak when its input is the corresponded code, otherwise, the decoder's output is formed by cross-correlation peaks since its input is an interfering code from another user. Errors occur in the decoder whenever the desired user transmits a bit " 0 ", and all the nonzero temporal positions of the desired code are filled up with pulses of interfering users. Further details regarding error events are given in Section 2.4. The short amplitude peaks that appear during the autocorrelation process of the desired signal are due to the ODLs scheme, shown in Figure 2.3c. Usually, a MWML-OOC set is defined by the quadruple $\left(L, W, \lambda_{a}, \lambda_{c}\right)$ with unipolar $(0,1)$ sequences, where $\lambda_{a}$ and $\lambda_{c}$ are the maximum value of the autocorrelation sidelobes that represents all the nonzero temporal shift of the autocorrelation function, and the maximum value of the cross-correlation function, respectively. A MWML-OOC with $\lambda_{a}=\lambda_{c}=1$ can be classified as codes with good correlation properties and is represented in short as $(L, W, 1)$.
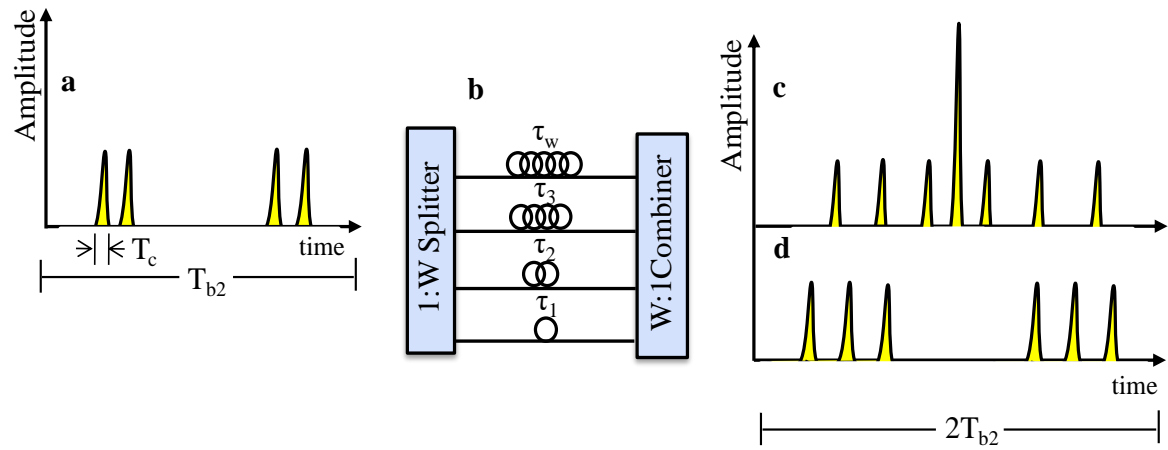

Figure 2.3. 1-D MWML-OOC decoding scheme based on intensity correlation. a) Received signal is sent to the decoder. b) Possible decoder scheme based on ODLs, splitter and combiner. c) Autocorrelation signal plus interference from other users. d) Cross-correlation signal. Picture adapted from [1]. 


\section{FLEXIBLE OCDMA NETWORKS}

Each data information bit is encoded in a waveform $s(t)$ that corresponds to code sequences. Furthermore, each receiver correlates the mask of this code, $f(t)$, with the sent signal, $s(t)$ [74]. The receiver output, $r(t)$ is a correlation operation which shows the similarity degree between them, and is given by

$$
r(t)=\int_{-\infty}^{\infty} s(\tau) f(\tau-t) d \tau
$$

The algorithms for code design generally seek to maximize the autocorrelation $s(t)=f(t)$, and minimize the cross-correlation between codes, $s(t) \neq f(t)$ to extract the maximum in orthogonality (degree of differentiation between them) and cardinality (quantity of available codes).

The general conditions for code orthogonality are [73]:

1) Each code sequence can be distinguished from the shifted version of itself;

2) Each version (possibly a shifted version) can be distinguished from other sequences of the code set.

Hence, let us express the above statements in mathematical form. For two sequences of length $L, X=\left\{x_{0}, x_{1}, x_{2}, \ldots, x_{L-1}\right\}$ and $Y=\left\{x_{0}, x_{1}, x_{2}, \ldots, x_{L-1}\right\}$, there are two results of discrete correlation that represent the interaction between them [21]

i) For sequence $X=x(k)$ :

$$
\left|Z_{x, x}\right|=\left|\sum_{i=0}^{N-1} x_{i} x_{i+k}\right|=\left\{\begin{array}{ccr}
W, & \text { for } & k=0 \\
\leq \lambda_{a}, & \text { for } & 1 \leq k \leq L-1
\end{array}\right\}
$$

ii) For each pair of sequences $X=x(k)$ and $Y=y(k)$ :

$$
\left|Z_{x, y}\right|=\left|\sum_{i=0}^{N-1} x_{i} y_{i+k}\right| \leq \lambda_{c} \text { for } \quad 1 \leq k \leq L-1
$$

where $Z_{x, x}$ and $Z_{x, y}$ correspond to the autocorrelation and cross-correlation, respectively, $\lambda_{a}$ as already mentioned is the autocorrelation lateral lobes (or also called out-of-phase autocorrelation) corresponding to shifted versions of the same sequence, and $\lambda_{c}$ is the cross-correlation lobes between different sequences. 


\subsection{2-D Encoding}

This subsection aims at introducing and explaining the advantages of different encoding schemes based on 2-D codes, in which the user's data bit is spread simultaneously in time and frequency domains.

The problems concerning the inefficiency in using bandwidth and power associated to the first implementations of 1-D codes can be mitigated by converting 1-D codes into 2-D codes via simultaneous manipulation of the time and frequency (wavelength) dimensions where their selections obey a code construction algorithm, which not only increases the flexibility of the code design, but also improves the cardinality dramatically while maintaining good auto- and cross-correlation properties.

The combination of frequency and time encoding provide better flexibility in code choice, resulting in an increased capacity of the system. Such flexibility allows for reduced code length design without hindering the code cardinality or the system's performance in relation to the 1-D codes [52]-[54], [75]. In addition, the frequency and time encoding can also reach long transmissions at high rates to such an extent that the linear dispersion becomes less critical. Furthermore, in 2-D encoding schemes, the code sequences are characterized by frequency hopping (FH). If a hop occurs in a time slot smaller than the information signal period the process is defined as fast frequency hopping (FFH), in which case fast jumps occur at wavelengths that change for every pulse of a given temporal sequence.

The difference between $\mathrm{FH}$ and $\mathrm{FFH}$ signals in terms of frequency hopping occupancy is depicted in Figure 2.4. Notice that as time advances, the signal occupies a separate frequency band as determined by the pseudo-random hopping code sequence. It can be further noted that hops in FFH must be to an exclusive frequency, i.e., to a frequency different from the previously utilized.

Optical FFH (or OFFH) is a promising 2-D coding technique to be implemented in flexible OCDMA networks with support to multirate transmissions, especially due to its practical implementation and all-optical passive signal encoding based on fiber Bragg gratings (FBGs) [55], [76]. Both frequency hopping techniques are described in more details in the next subsections. The terms OFFH and FFH are addressed interchangeably in this thesis.

Generally, a 2-D code can be represented by a $m \times n$ matrix, with the number of rows, $m$, associated to the available wavelengths, and the number of columns, $n$, related to the chip interval (code length). For example, let us consider two 2-D codes $x$ and $y$ that belong to code set $C$. Assuming orthogonality for different used wavelengths (neglecting interactions) implies that displacements will be considered only in time. 


\section{FLEXIBLE OCDMA NETWORKS}

Therefore, with $0<\tau<n$, the results for the discrete binary correlation functions are given by [52]

i) For nonzero shift autocorrelation of $x$ :

$$
Z_{x, x}=\sum_{i=0}^{m-1}\left(\sum_{j=0}^{n-1} x_{i, j} x_{i,(j+\tau) \bmod (n)}\right) \leq \lambda_{a}
$$

ii) For cross-correlation between $x$ and $y$ :

$$
Z_{x, y}=\sum_{i=0}^{m-1}\left(\sum_{j=0}^{n-1} x_{i, j} y_{i,(j+\tau) \bmod (n)}\right) \leq \lambda_{c}
$$

where $Z_{x, x}$ and $Z_{x, y}$ corresponds to the autocorrelation and cross-correlation, respectively, $\lambda_{a}$ is the out-of-phase autocorrelation peak corresponding to the lateral lobes and $\lambda_{c}$ is the cross-correlation peak.

The in-phase autocorrelation value is equal to the code weight, $Z_{x, x}(0)=w$. The cardinality depends on the code generator algorithm and the notation follows the standard identification $\left(m \times n, w, \lambda_{a}, \lambda_{c}\right)$ or $\left(m n, w, \lambda_{a}, \lambda_{c}\right)$ adopted for 2-D OOC, with the length given by $L=m n$. The correct decoding signal of a given user fundamentally depends on the proper implementation of this correlation function in the network.

a Frequency Hopping Signal

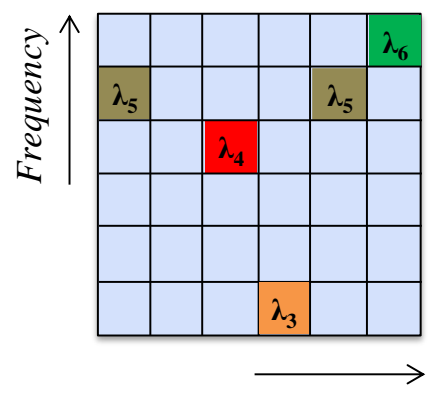

Time

\section{b Fast Frequency Hopping Signal}

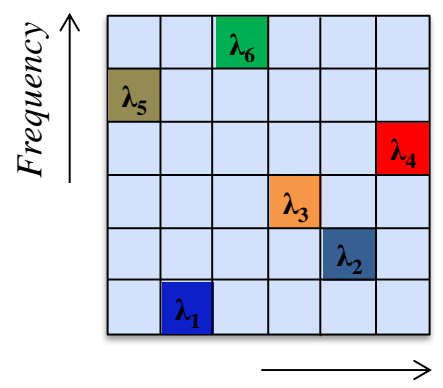

Time

Figure 2.4. Frequency hopping technique. a) Time and frequency occupancy of frequencyhopping (FH) signal. b) Time and frequency occupancy of fast frequency-hopping (FFH) signal. 


\subsubsection{Frequency Hopping, FH-OCDMA}

In this subsection, 2-D codes with support to multiservice multirate transmissions, as well as their encoders/decoders based on the FH scheme, is addressed. In this scheme, the pulses' allocation is carried out simultaneously in both time and wavelength following a previously established code design.

In frequency hopping, the carrier frequency of the transmitter hops in accordance with an apparently random pattern. This pattern is in fact a pseudo-random code sequence. However, the order of the frequencies selected by the transmitter is taken from a predetermined set as dictated by the code sequence, namely 2-D wavelengthhopping time-spreading (WH/TS) [51], [52], [75]. A 2-D WH/TS is a family of codes based on FH scheme capable of performing frequency spreading in time and wavelength domain simultaneously. It is worth mentioning that this code family covers many coding schemes proposed in the literature like, for example, prime code optical orthogonal code [77], multiple-wavelength optical orthogonal code [78], and one-coincidence frequency-hopping code/optical orthogonal code (OCFHC/OOC) [53].

The OCFHC/OOC stands out due to its good performance [79], [80] and good code generation features since the code length can be chosen regardless of the number of available wavelengths without sacrificing the code good correlation properties. 2-D codes provide a significant increase in code cardinality, allowing for a larger number of simultaneous users in the network. In addition, it provides greater flexibility in the choice of code parameters and thus are considered an interesting alternative to overcome the shortcomings of 1-D codes. Finally, 2-D codes have additional advantages such as low chip rate and network latency as well as high spectral efficiency, all due to its short code length [54] when compared to 1-D codes.

The originally proposed 2-D codes were based on equal code weights and code lengths with the assumption of providing the same transmission rate and level of service for all network's users. In addition, they had to comply with the code generation constraints such as low cross-correlation values. On the other hand, 2-D codes with different values of code weight and length have been recently proposed to achieve multiservice multirate transmissions. This type of 2-D codes allows for a modification of the users' code-weight and -length according to their transmission demands. For example, users with demand of higher transmission rates are allocated with shorter code lengths.

There are several 2-D code proposals with support to multiservice, multirate transmissions available in the literature [54], [81], but the recent proposed 2-D multiweight, multilength OOC (2-D MWML-OOC) is of particular interest since it allows the number of wavelengths, code-weight and -length to be chosen 


\section{FLEXIBLE OCDMA NETWORKS}

independently which, by its turn, allows arbitrary levels of data rates and QoS differentiation [54]. In addition to its useful design flexibility, the 2-D MWML-OOC has out-of-phase autocorrelation and cross-correlation bounded by one at the most and has a simple code generator. An example illustrating a network scenario using regular 2-D codes and 2-D codes with support to multiservice multirate transmissions is depicted in Figure 2.5.

All users are assigned codes with the same weight $(W=3)$ and length as shown in Figure 2.5a. This represents the case of 2-D OCFHC/OOC, normally employed in single-service and -rate OCDMA networks. Figure 2.5b, by its turn, shows users with assigned codes of different weight and length. For example, user \#1 has larger code length and lower code weight than user \#U. This case represents the 2-D MWMLOOC. Finally, it is worth pointing out that some temporal positions of chips are not filled and the same wavelength can be used more than once in the same code length. These features never happen in FFH schemes as will be discussed later.

Next, Figure 2.6 shows a possible scheme for implementation of 2-D MWML-OOC based on an all-optical encoding process. The encoder consists of a $1 \times W$ wavelengthdivision demultiplexer, $W$ ODLs, and a $W \times 1$ wavelength-division multiplexer that can be, for example, an arrayed waveguide grating (AWG) device. Moreover, the number of ODLs is equal to the code weight, and the delay of each line is equal to the nonzero position of the code. In the encoding process, a broadband short pulse with duration $T_{c}$ and high amplitude corresponding to data bit " 1 " is sent to the device to be encoded, as shown in Figure 2.6a. The incident pulse is then separated by the demultiplexer into $W$ pulses following a pre-established wavelength set, Figure $2.6 \mathrm{~b}$.

a Regular 2-D OOC

User \#1

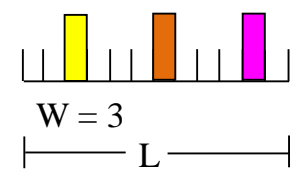

User \#U

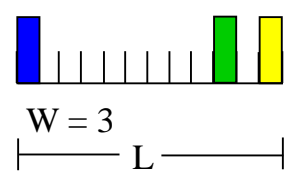

b 2-D MWML-OOC

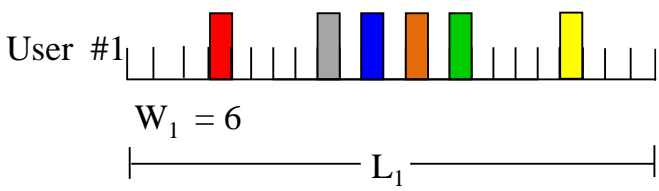

User \#U

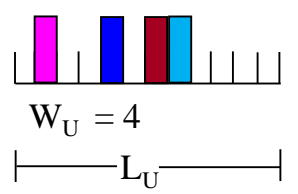

Figure 2.5. Example of 2-D OOC. a) All network's users assigned with regular 2-D OOC codes with equal code-weight and -length. b) Distinct users assigned with codes of different weight and code to achieve multiservice, multirate transmissions. 

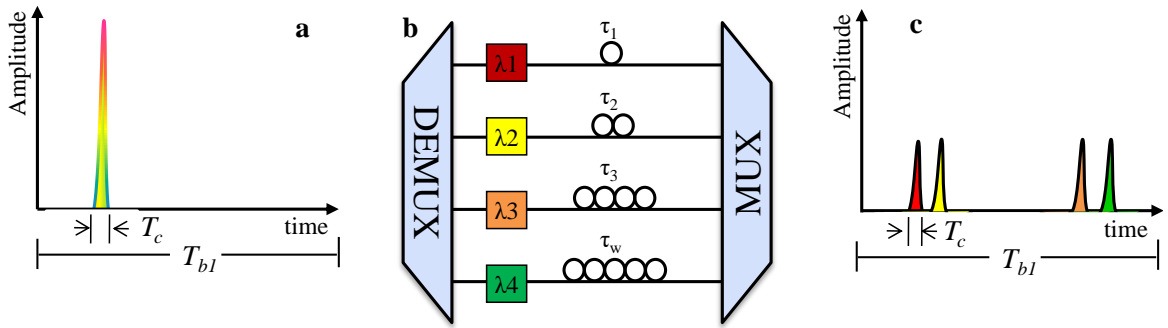

Figure 2.6. 2-D MWML-OOC encoding scheme based on time and frequency spreading. a) A broadband short pulse corresponding to data bit " 1 " is sent to the encoder. b) Possible encoder scheme based on ODLs, demultiplexer (DEMUX) and multiplexer (MUX). c) Encoded signal. Picture adapted from [1].

Then, after being split into $W$ pulses each with its corresponding wavelength, their respective ODLs generate a temporal delay following the features of the code sequence. In this example, the second pulse experiences a higher delay than does the first pulse. Afterwards, the pulses are combined by a wavelength-division multiplexer in the encoding output, as shown in Figure 2.6c. This process accounts for the encoding of 2-D MWML-OOC.

In the same fashion, the decoding process is depicted in Figure 2.7, where the received propagated signal, Figure 2.7a, goes through a decoder that consists of a $1 \times W$ demultiplexer, with $W$ delay lines, and a $W \times 1$ wavelength-division multiplexer. However, the ODLs are placed in a complementary configuration to that used in the encoder so that the time delays produced by the ODLs at the encoder and decoder are complementary to each other.

This scheme allows for an alignment of the pulses that compose the transmitted encoded signal, Figure 2.7b. When the decoder outputs a high amplitude peak as shown in Figure 2.7c, which means that the pulses were positioned correctly via the matched filter scheme, and represents a pattern defined by the autocorrelation function that allows the transmitted data bit to be correctly restored after optical-toelectrical conversion and threshold decision.

Otherwise, when the output of the decoder is cross-correlated, which means that the desired transmitted signal suffered interference and cannot be properly autocorrelated anymore, a background interfering signal defined by the crosscorrelation function is formed, Figure $2.7 \mathrm{~d}$. The appearance of low amplitude peaks shown in Figure 2.7c around the desired high amplitude peak during the autocorrelation operation (matched filter) of the desired signal are due to the ODLs. 

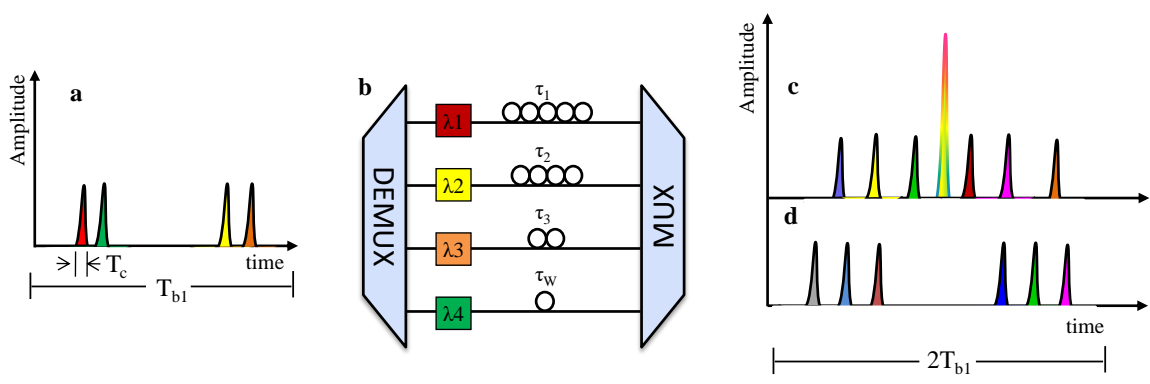

Figure 2.7. 2-D MWML-OOC decoding scheme based on time and frequency spreading. a) Transmitted encoded signal is sent to the decoder. b) Possible decoder based on ODLs, demultiplexer (DEMUX) and multiplexer (MUX). c) Output autocorrelation signal. d) Output cross-correlation signal. Picture adapted from [1].

\subsubsection{Fast Frequency Hopping, FFH-OCDMA}

This subsection addresses a second kind of 2-D encoding scheme, in which jumps occur at wavelengths that change for every temporal chip of the code sequence. In addition to the explanation of FFH encoder/decoder based on MGBs, the differences between conventional FFH and multilength FFH are also addressed here.

The OCDMA network based on FFH scheme and multiple Bragg gratings (MBGs) encoders/decoders was originally proposed by Fathallah [40], [55] and called FFHOCDMA. In fact, the encoding process is based on a series of MBGs [82] to generate fast hopping frequency patterns. These gratings spectrally and temporally slice an incoming broadband pulse into several components, generating then optical frequency patterns. This attractive encoding scheme stands out not only due to its good performance [40], [65] but also for its practical feasibility based on optical intensity manipulation of the incoherent signal and detection at the receiver side via IM-DD approaches. In addition to its asynchronous transmission nature, the passive all-optical encoding scheme based on MBGs implies potential low-cost and robust implementation of FFH-OCDMA (or OFFH-CDMA) networks also for multirate transmissions purposes [76].

An inherent feature of the OFFH scheme, as already mentioned, is that the frequency changes at a significantly higher rate than the information rate, which means that each pulse in a code sequence is transmitted at an exclusive wavelength. The OFFH scheme was initially proposed for single rate transmissions and assigned equal code lengths to all users so that the same data transmission rate was provided. 
The first OFFH-CDMA network with support to multirate transmissions was proposed later by Inaty et al. [47], [56]. The authors employed incoherent MBGs to perform the encoding process and achieved multiple rates through different code lengths while keeping constant the chip period. The different code lengths, entails in encoders and decoders with different quantity of Bragg gratings, where a short number of gratings is designed to high rate transmissions while a large number of gratings is designed to low rate transmissions.

An example shall illustrate a scenario of regular 2-D codes for single rate transmissions and 2-D codes with support to multirate transmissions. In the codes depicted in Figure 2.8 each temporal slot is filled with a pulse at an exclusive wavelength. All users of a regular $U$-users network are assigned to codes having the same weight and length, as shown in Figure 2.8a. The multirate scenario, by its turn, has each user assigned to a code of different length so that a specific transmission rate can be achieved. For example, in Figure $2.8 \mathrm{~b}$, user \#U is assigned to a code half as short as that of user \#1, consequently achieving twice the data transmission rate of user \#1. Accordingly, the bit period changes for each code of the multilength scheme and the chip period remains constant and the same for all codes.

From a different perspective, variating the bit period in conformity with the required transmission rate can be seen analogously as modifying the code length. Moreover, changing the bit period is analogous to changing the total round-trip time (RTT) of the pulses inside the MBGs encoder/decoder. The RTT represents the time that all pulses of a single code require to be reflected and exit the MBGs. In this way, changing the number of gratings of the MBGs structure, which intrinsically changes the code length, will eventually variate the bit period. Therefore, decreasing the total RTT results in a temporal decrease of the code duration, which is analogous to reducing the code length and, consequently, increasing the transmission rate.
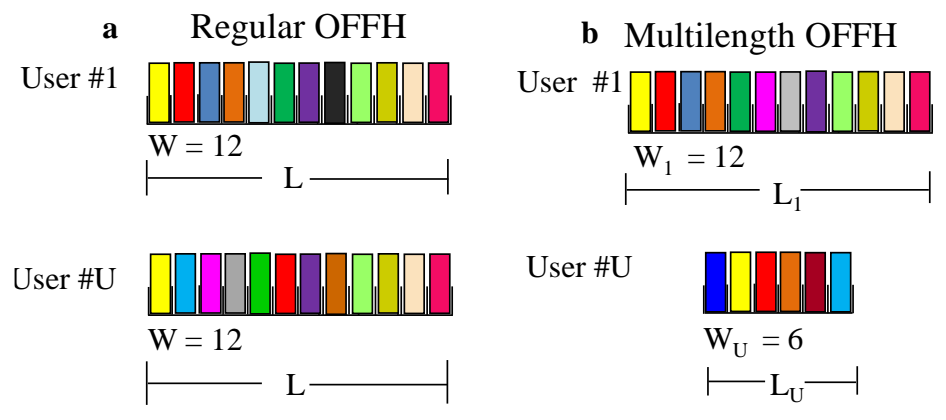

User \#U

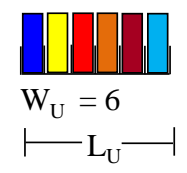

Figure 2.8. Example of FFH-based multilength codes. a) Regular OFFH scheme assigning codes with the same length for all network's users. b) Multilength OFFH scheme assigning larger codes to low-rate users and shorter codes to high-rate users. 
To better illustrate this concept, a possible network architecture based on the OFFHCDMA scheme to support multirate transmissions employing MBGs with different number of gratings, is illustrated in Figure 2.9. It is considered in this scenario, MBGs encoders with 12 gratings (low-rate transmission), 6 gratings (medium-rate transmission) and 4 gratings for high-rate transmissions. The MBGs structures that compose the users' encoders are arranged in series and designed to reflect (in this example) the following 12 wavelengths $\lambda_{3}, \lambda_{5}, \lambda_{1}, \lambda_{10}, \lambda_{8}, \lambda_{4}, \lambda_{11}, \lambda_{6}, \lambda_{12}, \lambda_{9}, \lambda_{2}$, and $\lambda_{7}$ for a user of low-rate transmission, the following 6 wavelengths $\lambda_{25}, \lambda_{13}, \lambda_{28}, \lambda_{18}, \lambda_{15}$, and $\lambda_{20}$ for a medium-rate user and the following 4 wavelengths $\lambda_{25}, \lambda_{13}, \lambda_{15}$, and $\lambda_{20}$ for the high-rate transmission user, respectively. The fourth MBGs structure is only shown for illustration purposes, see Figure 2.9b. Notice that the frequencies (wavelengths) cannot be used more than once into the same code sequence [55].

Initially, a broadband short pulse modulated with OOK, which represents the users' data bit, is generated. Then, this broadband pulse, shown in Figure 2.9a, is sent to the MBGs-based encoder when the value of the users' data bit is " 1 ", otherwise no power is sent for the transmission of data bit " 0 ". Subsequently, the MBGs generate independent frequency pulses and place each of them in an appropriate time-slot, depicted in Figure 2.9b and Figure 2.9c, respectively, following a scheme previously established by the OFFH-based code generator. The output signal established by the wavelength reflections of the MBGs forms the code sequence and is ready to be transmitted over the network, see Figure 2.9c. In this example, the network uses single mode fiber (SMF) and only the code corresponding to the high-rate user is shown for the sake of space. Furthermore, the Bragg gratings produce the frequency spectrum slicing while the temporal position of the Bragg gratings produces the respective time delays in a similar fashion to the ODLs in 1-D coding. Indeed, the time-frequency pulses are determined by the order in which they are tuned to the respective Bragg gratings following the concept of first-in, first-reflected [40].

It is worth pointing out that the chip duration and the number of gratings in the encoder establishes the nominal data bit rate of the network, i.e., all reflected pulses of a data bit should leave the encoder before the next bit's pulses enter [83]. Moreover, the impulse response of each Bragg grating is defined as the inverse Fourier transform of the grating complex reflectivity, where the incident pulses associated with the transmitted data are normally much narrower than the response duration of the grating. This encoding process is based on a convolution of an incoherent short pulse modulated by the data source with the response of each Bragg grating.

At the decoder side, Figure $2.9 \mathrm{~d}$, the wavelengths are placed in the reverse order of those in the encoder to accomplish the decoding function. Then a matched filter based-decoder removes the translation between the wavelengths and realigns all pulses from the received signal into a single pulse as illustrated in Figure 2.9e. 


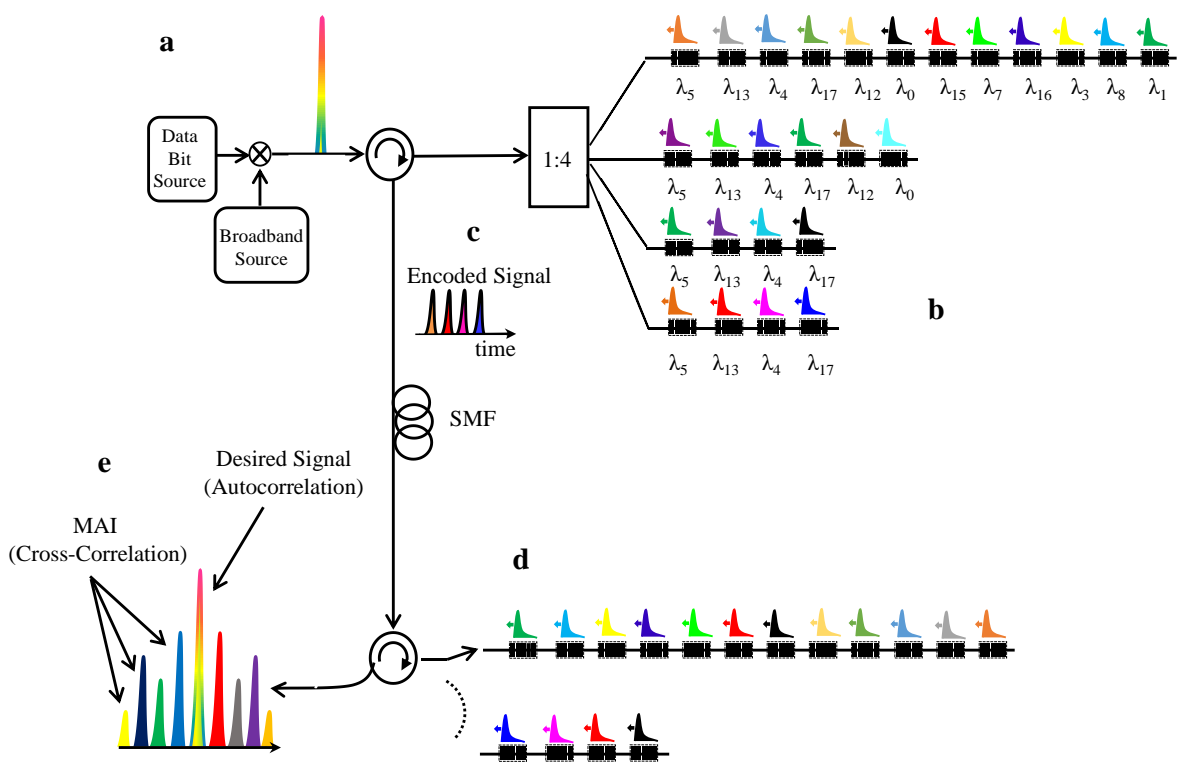

Figure 2.9. Possible implementation of the FFH scheme based on MBGs. a) A broadband short pulse corresponding to data bit " 1 " is sent to the encoder. b) The MBGs reflect the signal encoding simultaneously in sequential time slots and disjoint wavelength subbands according to the respective wavelengths in the Bragg grating by the MBGs-based encoder. c) The encoded timefrequency sequence is ready to be transmitted. d) MBGs-based decoder with gratings placed in the reversed order. e) Autocorrelation signal plus interference and cross-correlation signals. SMF: single mode fiber. Picture adapted and based on a setup of single rate networks from [76].

Assuming the pulses are positioned correctly, they form a well-defined pattern given by the autocorrelation property (similarity level between the transmitted and received desired signal). If they are not, the pulses form an interfering background signal defined by the cross-correlation property representing the MAI as shown in Figure 2.9e. Lastly, at the receiver (not illustrated here), the decoding process is based on the sum of powers, where the final generated electric current is directly proportional to the incident optical intensity based on the quadratic detection law.

In OFFH coding scheme, the available bandwidth is subdivided into contiguous frequency intervals, where the transmitted signal occupies a frequency range in each temporal chip $T_{c}=2 \times n_{e f f} \times L_{s} / c$, where $L_{s}$ is the sum of one grating length plus one spacing distance between an adjacent grating, $n_{\text {eff }}$ is the effective index, and $c$ is the light speed [47]. Since the Bragg gratings are equally spaced, i.e., $T_{c}$ is constant, the pulses are correspondingly spaced at time intervals $T_{c}$ seconds apart from each other, which corresponds to the RTT between two consecutive gratings [40]. 


\section{FLEXIBLE OCDMA NETWORKS}

Furthermore, the bandwidth associated with the data bit rate is $B=1 / T_{b}$, and $T_{b}=$ $2 \times(L-1) n_{e f f} \times L_{s} / c$, where $L$ is the code length (also equivalent to the number of gratings) [47]. The gratings are written at the wavelength $\lambda_{B}$ (Bragg wavelength) and tuned to different wavelengths $\left(\lambda_{1}, \lambda_{2}, \ldots, \lambda_{F}\right)$, which are allocated in time intervals in accordance with the adopted algorithm. The parameter $F$ accounts for the total number of available wavelengths. The Bragg wavelength of each grating $\left(\lambda_{1}, \lambda_{2}, \ldots\right.$, $\lambda_{F}$ ) should thus comply with the code needs, and be allocated in time intervals according to an OFFH-based algorithm.

In addition, fiber Bragg gratings also offer the possibility of tuning the Bragg wavelength, which is very attractive for reconfiguring the encoder/decoder. Accordingly, wavelengths from longer code sequences can be tuned out of the working bandwidth of the OFFH network, therefore resulting in codes of different lengths and, consequently, supporting multirate transmission. Each Bragg grating can be individually tuned to adjust an intended wavelength from an available wavelength range. The wavelengths tuned out of the working bandwidth are no longer reflected by the Bragg gratings of the encoder/decoder [47], i.e., the gratings become transparent to these wavelengths. Finally, each of these grating contributes to a single reflected pulse. The number of available frequencies is limited by the tunability of the gratings, which establishes the system capacity.

A possible way of designing practical OFFH-based codes is by using the code generator based on the Bin's algorithm [84]. This algorithm employs $F \geq L$ for the code set generation. Furthermore, the codes are classified as one-coincidence sequences [40], and are characterized by the following three properties: 1) all the sequences have the same length; 2) each frequency is used at most once in each sequence; 3) the maximum number of hits between any pair of sequences for any time shift equals one. These codes were originally proposed with equal lengths to achieve single rates. Despite this, a possible way to obtain OFFH-based codes with different length is by (shorten) splitting longer codes to have shorter code sequences. Even though these codes have different lengths, they still preserve good correlation properties [47] as originally proposed [84]. 


\subsection{Multiple Access Interference}

In this subsection, one of the most deleterious sources of noise in conventional and flexible OCDMA networks is addressed and explained. A network scenario depicting how errors occur is illustrated. In OCDMA networks each user has a unique code sequence so that they can transmit data bits through the channel simultaneously. The different coding schemes currently available for 1-D codes [42] and 2-D codes [54], [85] seek to achieve larger cardinality and orthogonality. Nevertheless, using codes with good orthogonality do not ensure total differentiation between the users, as the manipulation of optical pulses occurs by signal intensity [86]. For example, let us consider a 2-D OCDMA network which uses temporal displacements of optical pulses in several wavelengths, arranged according to a specific code algorithm. These pulses are arranged according to a specific algorithm that assigns the code to each user. The information of each user is encoded using a specific signature sequence, multiplexed and sent through the optical channel to all users at the receiver side simultaneously. Each receiver contains the replica of the code attributed to each corresponded user, which enables the information to be extracted via a correlation operation. At the receiver side, the pulses of different wavelengths overlap to form an autocorrelation peak of high intensity if the corresponding user to the code contained in the receiver sends some information. Otherwise, the pulses in different wavelengths will remain far apart and low cross-correlation signals will be generated. Normally, the network accommodates several users simultaneously and the crosscorrelation signals can eventually accumulate and generate an interfering power comparable to the autocorrelation peak [21], [73]. This MAI can cause a significant increase of the BER and, consequently, of the packet throughput hence severely limiting the capacity of users to be served by the network.

Figure 2.10 illustrates how the MAI of various simultaneous users influences the signal of the desired user or user of interest. For this example, the users are defined in a five-class network, where each user class has a different code length and bit period, and thereby different transmission rates. In addition, each user class has its own code weight, characterizing also a multiservice scenario. Then, five users have their data bits encoded by specific codes based on 2-D MWML-OOC. The 2-D MWML-OOC features good correlation properties ensuring that a maximum of one hit occurs (overlap of two chips at the same time and wavelength) between two encoded signals for each asynchronous access in the network. Furthermore, asynchronous transmissions can be easily identified in this example as each sequence of the users does not occur at the same time. Consequently, overlap among chips of the users occur naturally after accessing the channel. Figure 2.10 illustrates the transmission of 4 data bits $\{1,0,1,1\}$ by the desired user along with the simultaneous asynchronous access of four interfering users with the aim of showing the errors caused by MAI. 
Before MAI

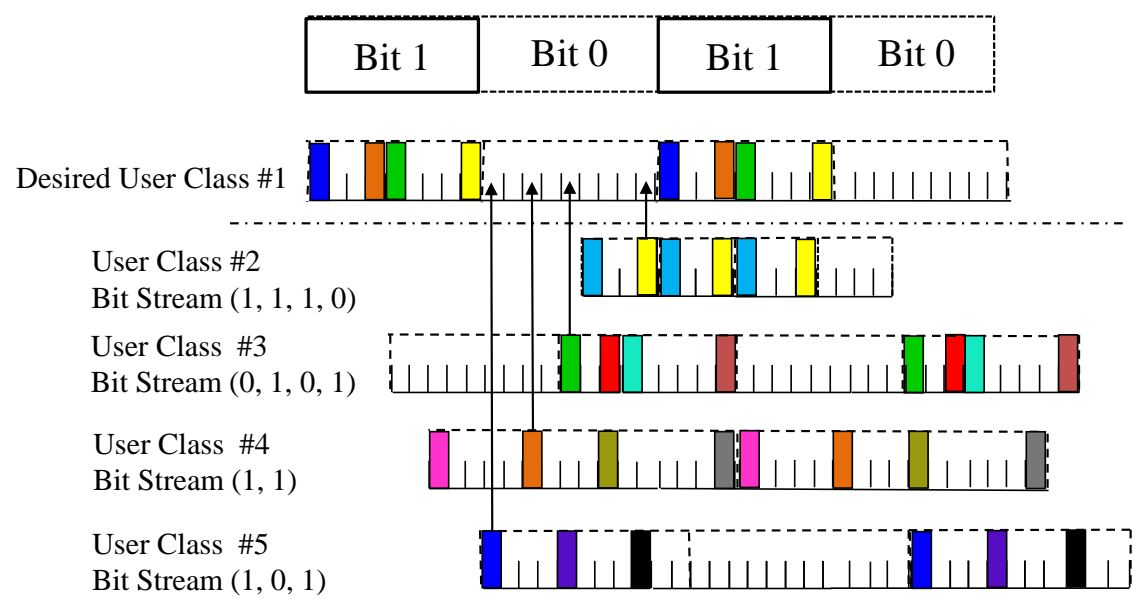

After MAI

\begin{tabular}{|c|c|c|c|}
\hline Bit 1 & Bit 1 & Bit 1 & Bit 0 \\
\hline
\end{tabular}

Figure 2.10. Example of the MAI contribution on the desired user class \#1 in a flexible OCDMA network with four interfering users.

Consider the desired user \#1, whose code has pulses at chip positions $(1,4,5,9)$. Errors by detection occur whenever the desired user transmits a bit " 0 ". Note that user $\# 2$ interferes on user $\# 1$ at the chip position 9. Likewise, users \#3, \#4, and \#5 also interfere on user \#1 at chip positions 5, 4, and 1, respectively. Noticeable, hits from the interfering users occur in both time and wavelength. The black vertical arrows indicate the affected chip positions of the user \#1. These hits contribute to errors in the receiver of the desired user due to the additional power generated by MAI, which will appear in the decision window after the autocorrelation operation of the desired user signal. MAI can increase in the same proportion as the number of simultaneous users in the network.

Finally, it is worth mentioning that MAI does not cause errors when the desired user transmits a data bit " 1 " since the interfering pulses are expected to increase the channel energy, which might make it impossible to detect a bit " 0 " during the transmission of a bit " 1 ". However, it only happens when the optical channel is considered positive and additive with no cancelation of energy, nonlinear effects of the channel are not considered, and the optimum detection threshold used in the network is equal the code weight of the desired user. 


\subsection{Multirate Techniques}

In this subsection, it is described the two most used techniques to achieve multirate transmissions in OCDMA networks. The first technique to be addressed here is called multilength, and the second technique is called multicode. They present different concepts and involve different schemes for encoders and decoders. Despite each of them having its own benefits in practical conditions, the multilength technique stands out since it requires only a single pair of encoder and decoder rather than the several needed for implementing the multicode technique, as will be described below. A performance comparison in terms of BER and packet throughput between both techniques is presented in Section 4.6.

In the first technique, referred to as multilength technique, every user of the network is assigned an exclusive code sequence, where the length of the code is varied to achieve arbitrary multiple rate transmissions. For example, if a user desires to transmit at high data rates, a single and short code length is assigned to this user. Figure 2.11a illustrates schematically this network scenario, where class- $j$ ' users have shorter code length than class-J users. In contrast, for the second technique, referred to as multicode technique, several codes with the same code length are assigned to a single user so that it can achieve arbitrary multirate transmissions [87]-[91]. Putting it simply, a set of code sequences with the same length is assigned to a user. For example, if a user desires to transmit at high data rates employing the multicode technique, several codes (times the network basic bit rate) are assigned to this user, all with the same length. The scheme of this technique is illustrated in Figure 2.11b, where each class- $j$ ' users are assigned with two codes.

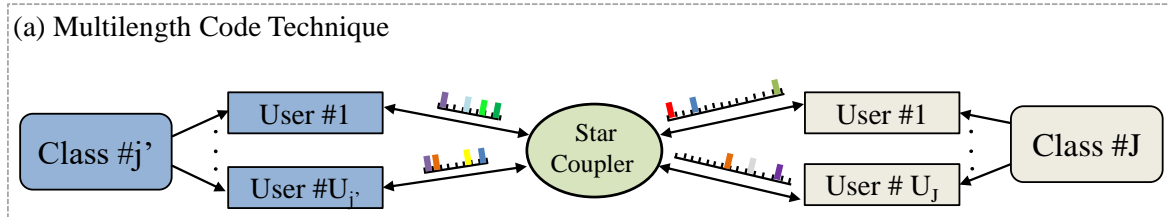

(b) Multicode Technique

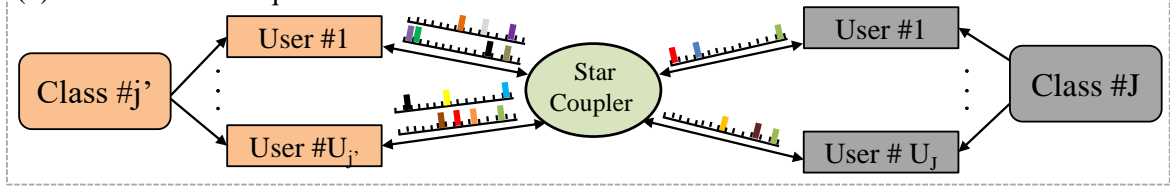

Figure 2.11. Scheme of the multirate OCDMA network connecting all J-class users in a star topology via optical fibers and a passive star coupler. Each user class has its own code parameters specifications. A two-class network is considered for the sake of space. a) Multilength code technique, where class- $j$ ' has shorter code than class- $J$. b) Multicode technique, where to each class- $j$ ' user two codes are assigned whereas to each class- $J$ user only one code is assigned. Picture taken from [109]. 


\section{FLEXIBLE OCDMA NETWORKS}

In Figure 2.11, only two-class users are considered for the sake of simplicity. A multirate OCDMA network based on the multilength code technique is illustrated in Figure 2.11a whereas a multirate network based on the multicode technique is illustrated in Figure 2.11b. Both networks consist of $J$-class users that share the same optical medium, where the desired user class is denoted as $j^{\prime}$. In Figure 2.11, only two-class users are considered for the sake of simplicity. A multirate OCDMA network based on the multilength code technique is illustrated in Figure 2.11a whereas a multirate network based on the multicode technique is illustrated in Figure $2.11 \mathrm{~b}$. Both networks consist of J-class users that share the same optical medium, where the desired user class is denoted as $j^{\prime}$.

In the multilength technique, for each user class $j \in\{1,2, \ldots, J\}$ of the network, see Figure 2.11a, the users' data rate is defined according with its given code length in a manner that high rate users have smaller code length and low rate users have longer code length such that $L_{1}<L_{2}<\cdots<L_{j}<\cdots<L_{J}$. However, in the multicode technique, for each user class $j \in\{1,2, \ldots, J\}$ of the network, see Figure $2.11 \mathrm{~b}$, the users' data rate is defined according with the number of $B_{j}$ assigned codes in a manner that high rate users are assigned to several codes and low rate users are assigned to only a single code each. Therefore, high data rate users transmit at $B_{j}$ times the network basic bit rate [92]. Moreover, user classes employing the multicode technique transmits $B_{j}$ times more packets than regular users of the same network. An example of traffic scenario with packet transmissions from both classes for the multicode technique is illustrated in Figure 2.12. It can be observed that class- $j^{\prime}$ (highrate users) transmits several packets simultaneously whereas class-J (low-rate users) transmits a single packet only.

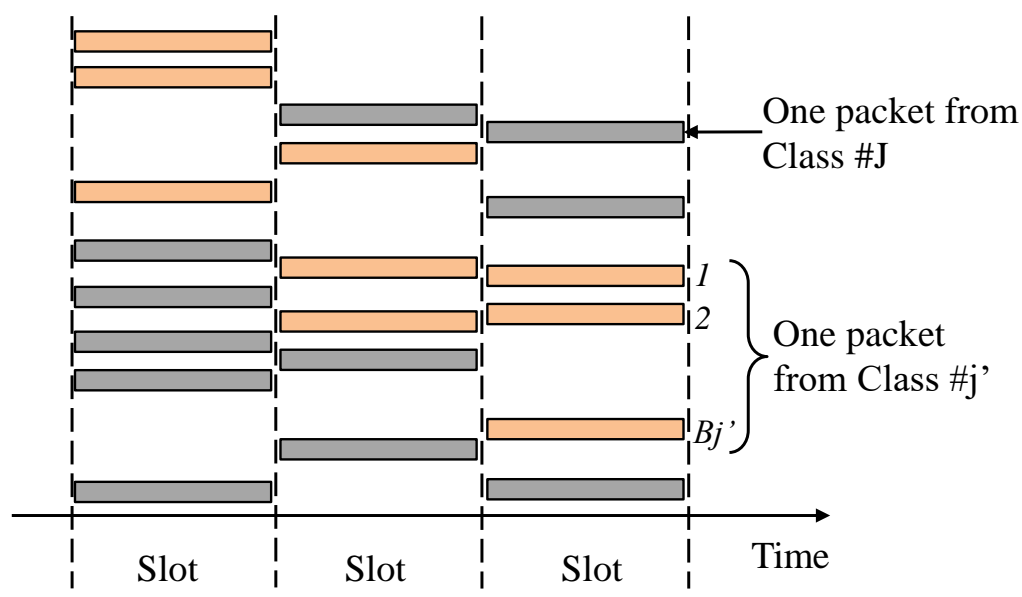

Figure 2.12. Example of the packet traffic transmission for both users' classes employing the multicode technique. Picture adapted from [91]. 
Next, Figure 2.13 shows the block diagram of possible transmitters' scheme for the implementation of both multirate techniques. We only consider a single user transmission from the desired class- $j^{\prime}$, for the sake of simplicity. Initially, the transmitter of the multilength technique receives modulated serial data bits to be transmitted, as shown in Figure 2.13a. Then, the data is encoded via a single encoder based on the features of this user, such as transmission rate and level of QoS. The encoded signal is finally combined with signals from other users and then transmitted through the network. In contrast, for a single user transmission, the transmitter of the multicode technique uses several encoders, as shown in the block diagram of Figure 2.13b. Observe that the transmitter receives modulated parallel data bits that go into their respective OCDMA encoder. Then, the output of the encoders is coupled via a passive star coupler and transmitted over the network. The encoder procedure is explained in detail in Subsections 2.2.1 and 2.3.1

At the receiver side, a single decoder (that acts as a matched filter in agreement with the code sequence of the desired user) is employed to retrieve the transmitted data for the multilength code technique. The block diagram of this technique is shown in Figure 2.14a. However, several parallel decoders $\left(B_{j^{\prime}}\right)$ are employed for the multicode technique. In this technique, each decoder matches one of the several $\left(B_{j \prime}\right)$ codes assigned to the desired user. The block diagram of the multicode receiver structure is illustrated in Figure 2.14b. The output of the decoders goes into a parallel-to-serial $(\mathrm{P} / \mathrm{S})$ converter so that the parallel data stream can be converted into serialized bits. The $\mathrm{P} / \mathrm{S}$ converters can be implemented for example using low cost fieldprogrammable gate array [93], [94].

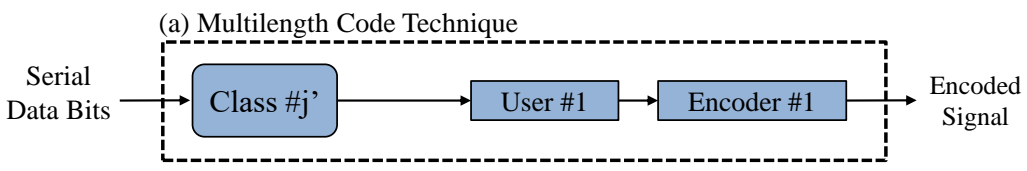

(b) Multicode Technique

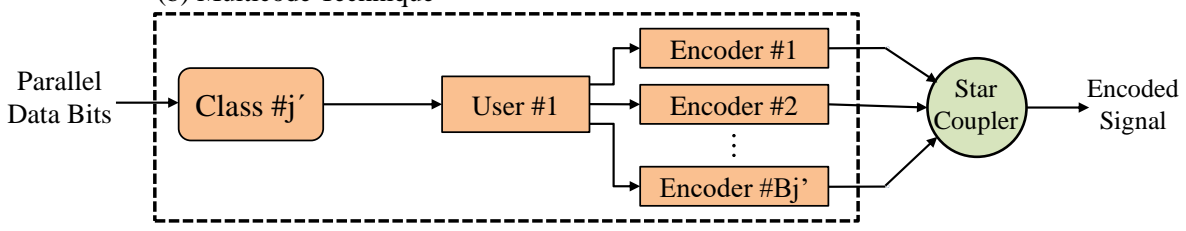

Figure 2.13. Block diagram of the transmitters considering the important stages for both multirate techniques. a) Multilength code technique employing only a single encoder. b) Multicode technique employing several encoders for a single user. Picture adapted from [90]. 
(a) Multilength Code Technique

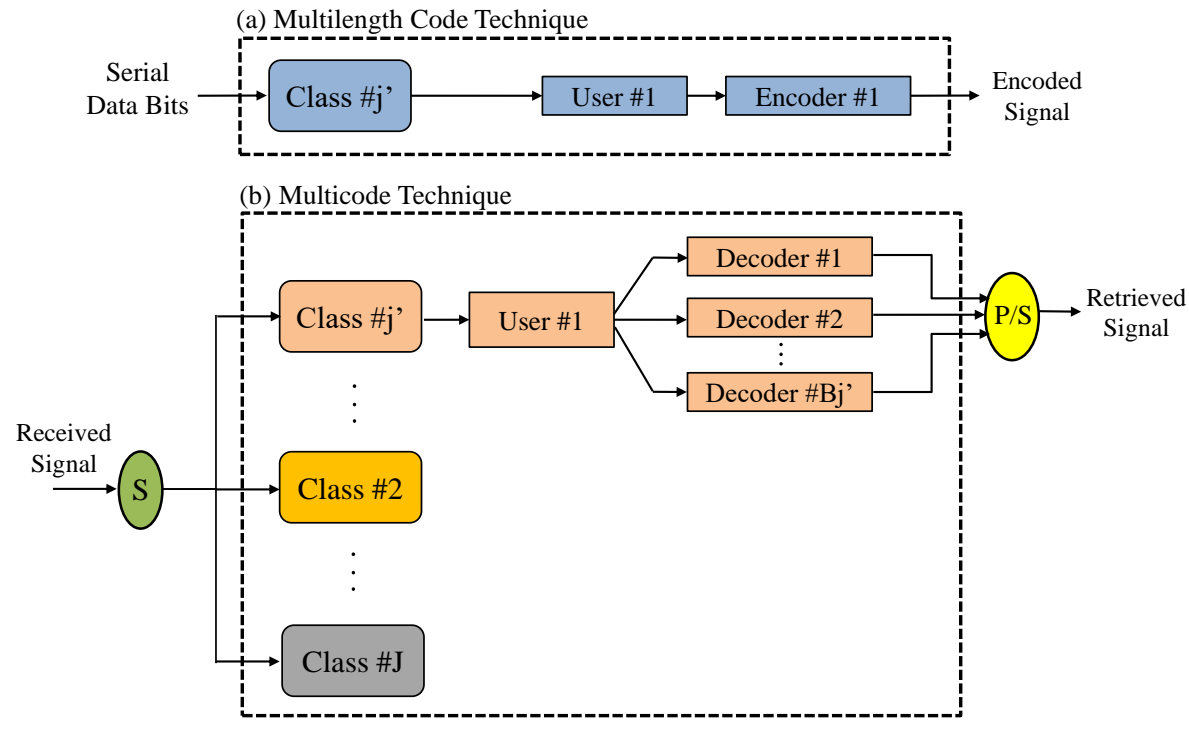

Figure 2.14. Block diagram of the receivers considering the important stages for both multirate techniques. a) Multilength code technique employing only a single decoder. b) Multicode technique employing several decoders for a single user. Picture adapted from [90]. 


\section{Chapter 3 \\ Mathematical Formalisms}

In this chapter, we introduce the new mathematical formalisms and analytical models developed for performance evaluation of flexible OCDMA networks with support to multirate and multiservice transmissions. Specifically, we derive new expressions for evaluating the BER, PER, packet throughput and packet delay performance, respectively. We model and describe in detail all the features of a flexible OCDMA network. Fiber impairments and other sources of noise inherent to the optical channel are ignored, while only MAI is assumed as the degrading factor of the network. The focus of this work is on the multirate and multiservice capability support in OCDMA networks. Nonetheless, additive noises can be easily included in the BER formalisms and, consequently, in the throughput formalisms as well.

It should be emphasized that the proposed mathematical formalisms only require the code parameters to be provided and not the users' code themselves, which makes the network analysis straightforward. In addition, the developed formalisms account for a general and average probability of interference between any pair of codes. Consequently, the formalisms can be used for network performance evaluation employing most available 1-D and 2-D multiweight multilength codes with maximum cross-correlation and out-of-phase autocorrelation values bounded by one. Finally, the developed analytical formalisms account for a general number of distinct users' classes present in the network. Hence, the formalisms can be successfully applied for performance evaluation of flexible OCDMA networks under any number of users' classes. Thus, the mathematical formalisms proposed in this thesis for flexible OCDMA networks represent an important contribution to the telecommunication field.

Part of the work presented in this chapter has been published as:

T. R. Raddo, A. L. Sanches, I. T. Monroy, and B.-H. V. Borges, "Throughput performance evaluation of multiservice, multirate OCDMA in flexible networks," IEEE Photonics Journal, vol. 8, no. 1, pp. 1-15, February 2016.

T. R. Raddo, A. L. Sanches, J. V. dos Reis Jr., and B. -H. V. Borges, "A new approach for evaluating the BER of a multirate, multiclass OFFH-CDMA system," IEEE Communications Letters, vol. 16, no. 2, February 2012. 


\section{MATHEMATiCAL FormaLismS}

T. R. Raddo, A. L. Sanches, I. T. Monroy, and B.-H. V. Borges, "Packet throughput performance of multiservice, multirate OCDMA in elastic networks", in Proc. IEEE International Conference on Communications (ICC) 2016, Kuala Lumpur, Malaysia.

T. R. Raddo, A. L. Sanches, I. T. Monroy, and B.-H. V. Borges, "Multirate IP traffic Transmission in flexible access networks based on optical FFH-CDMA" in Proc. IEEE International Conference on Communications (ICC) 2016, Kuala Lumpur, Malaysia.

T. R. Raddo, A. L. Sanches, B.-H. V. Borges, and I. T. Monroy, "Throughput performance analysis of multirate, multiclass S-ALOHA OFFH-CDMA packet networks," in Proc. IEEE ICTON, Budapest, Hungary, 2015.

T. R. Raddo, A. L. Sanches, J. V. dos Reis Jr., and B. -H. V. Borges, "Throughput evaluation of multirate, multiservice 2-D OCDMA packet networks," in Proc. IEEE ICTON, Graz, Austria, 2014.

T. R. Raddo, A. L. Sanches, J. V. dos Reis, Jr, and B. -H. V. Borges, "Multiservice, multirate IP transmission over OCDMA network," in Proc. IEEE ICTON, Cartagena, Spain, 2013.

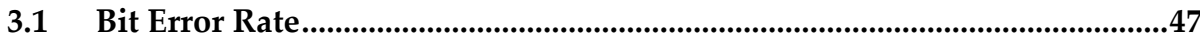

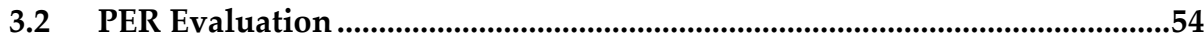

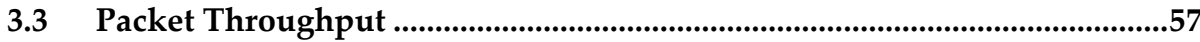

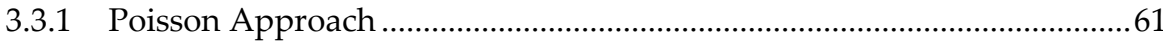

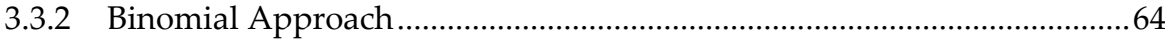

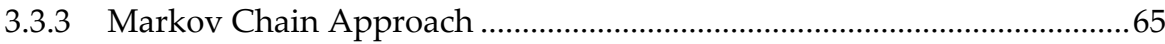

3.3.4 Packet Throughput for Multicode Technique .........................................67

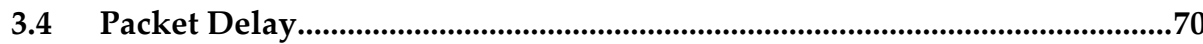




\subsection{Bit Error Rate}

In this subsection, we develop the mathematical formalism used for assessing the BER performance of flexible OCDMA networks. A new BER equation is derived assuming a binomial distribution for the MAI. A BER equation based on the gaussian distribution for the MAI is also derived. Finally, a BER equation considering a Poisson distribution for the MAI is adapted from the literature (for performance comparison purposes). It is noteworthy that the binomial-based BER equation accounts for error probabilities for both data bit " 0 " and " 1 " transmissions. In addition, this equation accounts for a general choice of threshold value, which allows one to calculate the optimum threshold detection of any network configuration. This can be very useful for optimization of the network performance.

Let us consider a flexible OCDMA network capable of achieving multirate and multiservice transmissions through different code lengths and arbitrary code weights, respectively [95], [96]. The block diagram of the flexible OCDMA network is shown in Figure 3.1. As depicted in the figure, each user of the network has a transmitter and receiver. At the transmitter side, the generated data bits from each user is converted to the optical domain (blue blocks) and then are modulated with a OOK modulator (brown blocks). Bit " 1 " is represented by the presence of an optical pulse while bit " 0 " is represented by the absence of optical pulses.

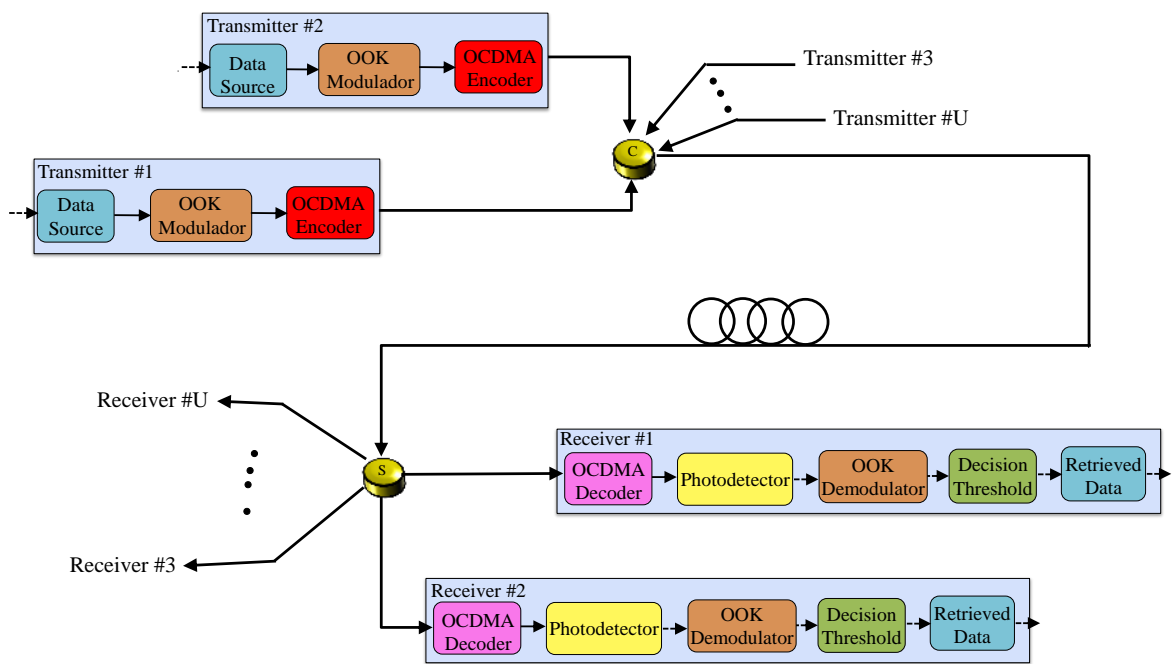

Figure 3.1: Block diagram of the flexible OCDMA network with support to multiservice, multirate transmissions, where users are connected in a star topology via optical fibers and passive star coupler/splitter. Only the more important stages are shown. C: coupler, S: splitter. 
Sequentially, the OOK-modulated signal goes through the encoding process and a unique code sequence is assigned to each user by the OCDMA encoder (red blocks). Succeeding the code assignment to users, the passive star coupler combines the output signals of the transmitters and provides access to the fiber channel. Despite the ideal channel adopted here, the simple superposition of users' signals in the combiner produces MAI, which is delivered to each user by the splitter. At the receiver side, the transmitted data bit along with MAI is decoded by the OCDMA decoder (pink blocks) by removing the temporal translation introduced in the encoder. After being decoded, the signal is directly detected by a photodetector (PD), where its electrical output signal is integrated over the chip period under a suited time slot, which is also part of the demodulation process (yellow blocks). The integrated signal is then compared to a threshold level previously stablished by a threshold decision device (green blocks). Accordingly, after these steps the signal recovery is finally completed.

Furthermore, the network consists of J-class users that share the same optical medium. The total number of users in the J-class network is $\mathrm{U}=\sum_{j=1}^{J} U_{j}$, where $U_{j}$ is the number of users in class- $j \in\{1,2, \ldots, J\}$. The network employs OOK modulation, where there is no signal during the bit " 0 " transmission. For bit " 1 " transmission, a pulse with amplitude given by real part of optical field is transmitted. Thus, the OOK signals carry the information in their amplitudes. Although the simplicity of OOK implementation is one of the main reasons for its use here, one can refer to [65] for networks based on advanced modulation formats.

For each class- $j$, the users' transmission rate is chosen by its corresponding code length given by $L_{1}>L_{2}>\cdots>L_{j}>\cdots>L_{J}$ in a way that high rate users have smaller code lengths and low rate users have larger code lengths, and $L_{j}=T_{j} / T_{c}$, where $T_{j}$ is the bit period of class-j users, and $T_{c}$ is the chip period. $T_{c}$ is assumed constant and the same for all classes $J$, and the transmission power of all users $U$ in all classes $J$ is normalized to unity [95].

In addition, the network supports multiservice (or equivalently diffServ) transmissions through specific code weights $W_{j}$ in a manner that the larger the code weight, the higher the QoS supported. Without any loss of generality, it is assumed that the desired user is the first user in the desired class denoted as $j^{\prime}$. Thus, the transmitted intensity-modulated optical signal of the $u$ th user in the $j$ th class will be

$$
S_{u, j}(t, f)=b_{u, j}(t) C_{u, j}(t, f),
$$

where $b_{u, j}(t) \in\{0,1\}$ is the baseband signal of the $u$ th user in the $j$ th class, $C_{u, j}(t, f) \in$ $\{0,1\}$ is the uth user's hopping pattern of the $j$ th class, $t$ and $f$ are the time and frequency associated with a signal. 
The signal is combined with signals from other users and transmitted. Then, after going through the network, each user's signal arrives at the receiver with the signal of the desired user degraded by MAI. Hence, the received signal at the input of the decoder is given by

$$
r(t, f)=\sum_{j=1}^{J} \sum_{u=1}^{U_{j}} S_{u, j}\left(t-\tau_{u, j}, f\right)
$$

where $\tau_{u, j}$ is the time delay associated with the $u$ th signal of the $j$ th class. We consider only MAI on the detection of the desired user's bit $b_{1, j^{\prime}}$. Hence, the decision variable at the matched filter output will be

$$
\begin{aligned}
& Z=\int_{0}^{T_{j^{\prime}}} r(t, f) C_{1, j^{\prime}}(t, f) d t \\
& Z=\sum_{u=1}^{U_{j^{-1}}} I_{\left(j=j^{\prime}, u\right)}^{1, j^{\prime}}+\sum_{j=j^{\prime}+1}^{J} \sum_{u=1}^{U_{j}} I_{\left(j>j^{\prime}, u\right)}^{1, j^{\prime}}+\sum_{j=1}^{j^{\prime}-1} \sum_{u=1}^{U_{j}} I_{\left(j<j^{\prime}, u\right)}^{1, j^{\prime}}+b_{1, j^{\prime}} W_{j^{\prime}},
\end{aligned}
$$

where $I_{\left(j=j^{\prime}, u\right)}^{1, j^{\prime}}, I_{\left(j>j^{\prime}, u\right)^{\prime}}^{1, j^{\prime}}$ and $I_{\left(j<j^{\prime}, u\right)}^{1, j^{\prime}}$ are the interferences from the equal, longer, and shorter code length classes, respectively [95]. $W_{j^{\prime}}$ is the user's code weight of class- $j^{\prime}$. Without any loss of generality, the total MAI can be given by the sum of the interferences from all users' classes, denoted as $I_{j^{\prime}}$. Therefore, (3.3) can be rewritten as

$$
Z=I_{j^{\prime}}+b_{1, j^{\prime}} W_{j^{\prime}}
$$

Next, to derive the BER expression is considered that the MAI has a binomial distribution, since the interference of OCDMA networks is binomially distributed [42], [43], [46], [54], [55].

Moreover, MAI is assumed as the only degrading factor of the network since it is normally considered the dominant noise source in OCDMA networks [40], [41], [65], while ignoring other sources of noise. It is assumed a chip synchronous scenario, which reflects the worse possible case for the system analysis [80], [97].

Then, the moment-generating function of the binomially distributed total interference $I_{j^{\prime}}$ is [42]

$$
M_{I_{j^{\prime}}}(t)=E\left[e^{t I_{j^{\prime}}}\right]=\prod_{j=1}^{J}\left(1-p_{j j^{\prime}}+p_{j j^{\prime}} e^{t}\right)^{N_{j}}
$$




\section{MATHEMATICAL FORMALISMS}

where $N_{j}$ is the number of interfering users in class $j$, and $p_{j j^{\prime}}$ is the total probability of interference caused by a code of class $j$ on a code of class $j^{\prime}$ and is written as [95]

$$
p_{j j^{\prime}}=\frac{W_{j} W_{j^{\prime}}}{2 L_{j} F},
$$

where $F$ is the total number of available wavelengths. The probability of interference reduces to $p_{j^{\prime} j^{\prime}}=W^{2} / 2 L F$ [98] for a single-rate network, where $W=W_{j^{\prime}}$ and $L=L_{j^{\prime}}$.

It is worth pointing out that for 1-D networks, $F=1$ in (3.6). It should be mentioned that the user code of class $j$ with $W_{j}$ chips out of $L_{j}$ chips have pulsed signal and can hit on any of the $W_{j^{\prime}}$ chips of the class $j^{\prime}$ users [95], [97]. Bear in mind that in OFFHbased networks, the code weight $W_{j}$ is equal to the code length $L_{j}$, thus $W_{j}=L_{j}$.

Furthermore, the parameter $F$ accounts for the number of frequencies used in the code design during the generation of 2-D codes. Finally, the term $1 / 2$ in (3.6) results from the equal probability of each user transmitting on-off pulses with OOK modulation.

Next, the mean and variance of the binomially distributed MAI are required to proceed with the BER derivation. The binomial distribution is used to model the number of successes $\mathcal{P}$ in a sequence of $U$ independent experiments. Since the signals that compose the MAI are independently distributed random variables, it allows one to obtain the mean and variance via the moments of the moment-generating function. Thus, the mean and variance of the MAI are given by

$$
\eta=M_{I_{j^{\prime}}}^{\prime}(0)=\sum_{j=1}^{J} N_{j} p_{j j^{\prime}},
$$

and

$$
\sigma^{2}=M_{I_{j^{\prime}}}^{\prime \prime}(0)-\left[M_{I_{j^{\prime}}}^{\prime}(0)\right]^{2}=(U-1) \mathcal{P}(1-\mathcal{P})
$$

respectively. Since the mean of such binomial MAI can also be given by $\eta=\mathcal{P}(U-1)$, we further obtain [95]

$$
\mathcal{P}=\sum_{j=1}^{J} \frac{N_{j} p_{j j^{\prime}}}{(U-1)},
$$

where $\mathcal{P}$ is the probability of interference of multirate, multiservice networks. 
Considering that the occurrence of data bits " 0 " and " 1 " are independent variables and their probability mass functions are represented by binomial distributions and consequently also the MAI has a binomial distribution, one can express the error probabilities when a bit " 0 " and " 1 " are sent, respectively, as

$$
P(\text { error } \mid 0)=\sum_{i=\mu}^{U-1}\left(\begin{array}{c}
U-1 \\
i
\end{array}\right) \mathcal{P}^{i}(1-\mathcal{P})^{U-1-i},
$$

and

$$
P(\text { error } \mid 1)=\sum_{i=0}^{\mu-1-W_{j^{\prime}}}\left(\begin{array}{c}
U-1 \\
i
\end{array}\right) \mathcal{P}^{i}(1-\mathcal{P})^{U-1-i},
$$

where $P($ error $\mid 0)$ and $P($ error $\mid 1)$ are both the probability of false alarm and the probability of false dismissal, respectively [43], and $\mu$ is the threshold value of the decision device.

In this way, considering that users transmit data bits " 0 " and " 1 " with equal probability $1 / 2$, assuming a general choice of $\mu$ and only MAI as degrading factor, the BER of class- $j^{\prime}$ users using both (3.10) and (3.11) can be calculated as [95]

$$
\begin{aligned}
& B E R\left(j^{\prime}\right)=P\left(Z \geq \mu \mid b_{1, j^{\prime}}=0\right) \cdot P\left(b_{1, j^{\prime}}=0\right)+P\left(Z<\mu \mid b_{1, j^{\prime}}=1\right) \cdot P\left(b_{1, j^{\prime}}=1\right) \\
& \operatorname{BER}\left(j^{\prime}\right)=\frac{1}{2}\left[P\left(Z \geq \mu \mid b_{1, j^{\prime}}=0\right)+P\left(Z<\mu \mid b_{1, j^{\prime}}=1\right)\right] \\
& \operatorname{BER}\left(j^{\prime}\right)=\frac{1}{2}\left[\sum_{i=\mu}^{U-1}\left(\begin{array}{c}
U-1 \\
i
\end{array}\right) \mathcal{P}^{i}(1-\mathcal{P})^{U-1-i}+\sum_{i=0}^{\mu-1-W_{j^{\prime}}}\left(\begin{array}{c}
U-1 \\
i
\end{array}\right) \mathcal{P}^{i}(1-\mathcal{P})^{U-1-i}\right],
\end{aligned}
$$

where $P\left(b_{1, j^{\prime}}=0\right)$ and $P\left(b_{1, j^{\prime}}=1\right)$ are the transmission probabilities of bits " 0 " and " 1, , respectively, and are considered equiprobable.

However, the BER expression can be reduced depending on the network scenario considered. For example, when the optical channel is considered additive and positive, with MAI assumed as the only noise source in the network, and a threshold value $\mu$ equals to the code weight of the desired user, the data bit " 1 " will always be decoded correctly. 


\section{MATHEMATICAL FORMALISMS}

In this fashion, no decision error will occur when a data bit " 1 " is sent, i.e., $P($ error $\mid 1)=0$. Thus, $(3.12)$ reduces to

$$
\begin{aligned}
& B E R\left(j^{\prime}\right)=P\left(Z \geq \mu \mid b_{1, j^{\prime}}=0\right) \cdot P\left(b_{1, j^{\prime}}=0\right)+P\left(Z<\mu \mid b_{1, j^{\prime}}=1\right) \cdot P\left(b_{1, j^{\prime}}=1\right) \\
& \operatorname{BER}\left(j^{\prime}\right)=\frac{1}{2}\left[P\left(Z \geq \mu \mid b_{1, j^{\prime}}=0\right)+P\left(Z<\mu \mid b_{1, j^{\prime}}=1\right)\right] \\
& \operatorname{BER}\left(j^{\prime}\right)=\frac{1}{2} \sum_{i=\mu}^{U-1}\left(\begin{array}{c}
U-1 \\
i
\end{array}\right) \mathcal{P}^{i}(1-\mathcal{P})^{U-1-i} .
\end{aligned}
$$

Remarkably, (3.13) can be considered as a general BER equation as it can be used for network performance evaluation of any 1-D and 2-D codes with good correlation properties. For 1-D codes, one should employ its corresponding code parameters and use $F=1$ in (3.6), due to the dimension of the code set in consideration [42]. In addition, (3.13) can be used for a general number of classes $J$ in the network.

Next, we obtain the BER equation of the flexible OCDMA network considering a gaussian distribution for the MAI. This expression will be assessed afterwards during the system performance comparison.

Even though the MAI has a binomial distribution, several works model it with a gaussian distribution to reduce the complexity of the mathematical formalism [43], [45]-[47]. Normally, this assumption is justified based on the central limit theorem which states that for a large number of simultaneous users in the network, their independently distributed random variables might converge to a gaussian distribution with a good approximation. To put it simply, if the number of users is large enough the gaussiandistribution might be a reasonable approximation to the binomial [43].

In this way, it is simple to obtain an approximation of (3.13) assuming a gaussian distribution for the MAI, $B E R_{G A}\left(j^{\prime}\right)$, as follows

$$
\begin{aligned}
& B E R_{G A}\left(j^{\prime}\right)=\frac{1}{2}\left[P\left(Z \geq \mu \mid b_{1, j^{\prime}}=0\right)+P\left(Z<\mu \mid b_{1, j^{\prime}}=1\right)\right] \\
& B E R_{G A}\left(j^{\prime}\right)=\frac{1}{2} Q\left(\frac{\mu-\eta}{\sigma}\right)
\end{aligned}
$$

where $\sigma$ is the square root of the variance, and $Q($.$) is the well-known Q$-function defined as [47] 


$$
Q(x)=\frac{1}{\sqrt{2 \pi}} \int_{x}^{+\infty} e^{-u^{2} / 2} d u
$$

A network scenario was investigated in [95], where it is shown that for flexible OCDMA networks the BER expression based on a gaussian distribution is not a reliable approximation for performance evaluation.

In contrast, when the number of users is large enough and the probability of interference among users' codes is small $\left(p_{j j^{\prime}}\right)$, the binomially distributed MAI might also converge to a Poisson distribution. Thus, we also introduce a BER expression based on Poisson distribution so that one can evaluate and compare the network performance under different MAI approaches. In this fashion, the Poisson distributed MAI becomes [42]

$$
P_{I_{j j^{\prime}}}\left(I_{j j^{\prime}}=n\right) \approx \frac{\left(U_{j} p_{j j^{\prime}}\right)^{n}}{n !} e^{-U_{j} p_{j j^{\prime}}}
$$

Note that (3.16) can be extrapolated to the total MAI $\left(I_{j^{\prime}}\right)$ since the sum of independent and identically distributed random variables also has a Poisson distribution. Hence, (3.16) can be cast as follows

$$
\begin{aligned}
& P_{I_{j^{\prime}}}\left(I_{j^{\prime}}=n\right) \approx \frac{\left(\sum_{j=1}^{J} U_{j} p_{j j^{\prime}}\right)^{n}}{n !} e^{-\sum_{j=1}^{J} U_{j} p_{j j^{\prime}}} \\
& P_{I_{j^{\prime}}}\left(I_{j^{\prime}}=n\right)=\frac{T^{n}}{n !} e^{-R} .
\end{aligned}
$$

Considering MAI as the only degrading factor of the network, equiprobable data, and $\mu$ as the threshold of the decision device, one can obtain the BER of class- $j^{\prime}$ users $\left(B E R_{P O}\left(j^{\prime}\right)\right)$ under the Poisson distribution for the MAI as [42]

$$
\begin{aligned}
\operatorname{BER}_{P O}\left(j^{\prime}\right) & =\frac{1}{2}\left[P\left(Z \geq \mu \mid b_{1, j^{\prime}}=0\right)+P\left(Z<\mu \mid b_{1, j^{\prime}}=1\right)\right] \\
\operatorname{BER}_{P O}\left(j^{\prime}\right) & =\frac{1}{2} P\left(I_{j^{\prime}} \geq \mu \mid b_{1, j^{\prime}}=0\right) \\
\operatorname{BER}_{P O}\left(j^{\prime}\right) & \approx \frac{1}{2}\left[1-e^{-R} \sum_{n=0}^{\mu-1} \frac{T^{n}}{n !}\right] .
\end{aligned}
$$




\subsection{PER Evaluation}

In this subsection, we describe the new network architecture based on OCDMA that supports multiservice and/or multirate IP traffic transmissions and then we develop the packet error rate (PER) and the packet throughput formalisms to be used for performance evaluation of IP flexible OCDMA networks, which are investigated in Sections 4.7 and 4.8. The analytical equations developed here are exclusive for performance evaluation of multiservice and/or multirate IP architectures and are adapted from the initially proposed formalism of single-rate coherent networks [99], [100].

The proposed architecture is extremely interesting from a practical point of view, since it does not require any new optical processing technology. Moreover, the new architecture of multirate IP traffic transmission is independent of the OCDMA scheme and spreading code employed, which means that coherent schemes and different types of codes can also be employed.

The block diagram of the proposed multiservice network is illustrated in Figure 3.2. The network supports $U$ users sharing the same optical fiber channel in a star topology. The number of transmitters and receivers are equal to the number of users. In addition, the transmitters and receivers have tunable optical encoders and decoders, respectively. The supported services and data rates of the users are directly related to the weight and length of the respective codes, i.e., the different code weights support multiservice (diffServ), and the different code lengths support data rate differentiation.

The encoding and decoding are performed solely in optical domain by ODLs structures, where the delay of the corresponding lines in the encoder and decoder are complementary to each other. At the transmitter side, every incoming IP packet is saved into a buffer after having its destination recognized. The network transmits onoff pulses employing on-off keying (OOK) modulation. Furthermore, the buffer is divided into $U$ first-in-first-out (FIFO) subparts, where IP packets that are destined to different receivers are stored in the respectively different subparts, i.e., IP packets that should be routed to the same receiver are saved in the same subpart sequentially. This procedure considerably decreases the encoder adjusting time, since encoders are adjusted to the number of packets that belong to the same user rather than being individually tuned for every incoming IP packet [99].

Then, the control unit records the total traffic of each subpart and sends the packets whenever the total traffic is larger than a predefined threshold. However, before sending the packets, the tunable optical encoder should be adjusted according to the desired address code. The outputs of the encoders are aggregated by an optical star coupler and transmitted to the receivers. At each receiver, a tunable optical decoder 


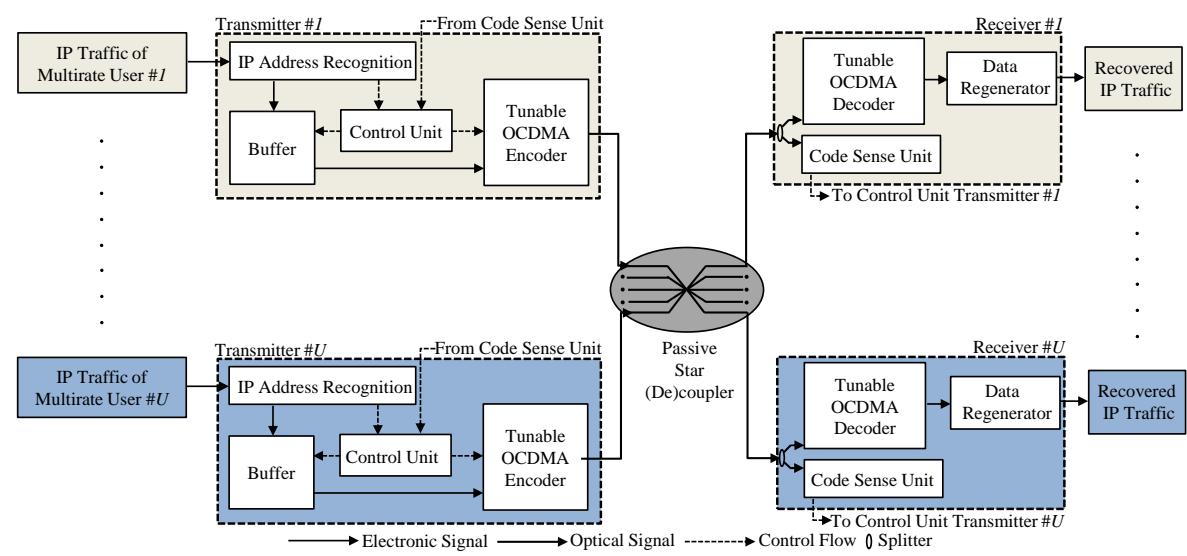

Figure 3.2: Block diagram of the proposed IP traffic architecture based on OCDMA with support to multiservice, multirate transmissions. Picture taken from [101].

is used to retrieve the desired code. Next, optical-to-electronic conversion is carried out via a photodiode (not shown in Figure 3.2) and the data bit is recovered by appropriately setting a threshold level at a threshold detector so that the original data stream can be regenerated.

Since a collision might occur whenever two or more transmitters send data at the same time to the same receiver, a code sense unit is used to avoid these collisions. It is noteworthy that each IP packet is buffered only twice at the edge of the optical network, then the buffer delay is significantly reduced if compared to traditional routing schemes, where IP packets are buffered at each network hop [99].

Then, we evaluate the PER assuming that MAI is the only degrading factor of the network. It is worth mentioning that the channel utilization of the network is directly related to the transmission rate in a way that the higher the channel utilization, the higher the transmission rate and, consequently, the higher the data traffic volume. In addition, it is worth pointing out that the higher the channel utilization, the higher will be the MAI. Accordingly, the channel utilization of class- $j$ can be written as [101]

$$
B_{j}=\frac{A B_{j}}{M B_{j}}
$$

where $A B_{j}$ is the average bit rate of class- $j$, and $M B_{j}$ is the maximum bit rate of class$j$. Also, the channel utilization of class- $j^{\prime}, B_{j^{\prime}}$, is given in the same way as (3.19), but substituting the class accordingly.

Next, we consider that users transmit data bits " 0 " and " 1 " with equal probability $1 / 2$. Since the users are sending data independently, the distribution of $U$ as the 


\section{MATHEMATiCAL FormaLismS}

number of active users sending IP packets is $U / T$, where $T$ is the total number of users accommodated in the network [99]. Thus, the probability that $U$ users are active sending IP packets in a network with $T$ users and their respective probability mass function becomes

$$
P_{a c}=\frac{1}{2} \sum_{j=1}^{J} \frac{U}{T} B_{j},
$$

and

$$
P_{i p(U)}=\left(\begin{array}{l}
T \\
U
\end{array}\right) P_{a c}^{U}\left(1-P_{a c}\right)^{T-U},
$$

respectively. Furthermore, the total BER of the number of active users $U$ can be expressed by the decoder BER (3.13) times the probability of error for $U$ active users $\left(P_{i p(U)}\right)$. Thus, the total BER of class- $j^{\prime}$ users, $P_{T}\left(j^{\prime}\right)$, can be expressed as

$$
P_{T}\left(j^{\prime}\right)=\sum_{u=1}^{U} P_{i p}(u) \cdot B E R\left(j^{\prime}\right)
$$

One can finally obtain the PER of class- $j^{\prime}$ for multiservice multirate IP traffic transmissions over OCDMA networks, considering a packet length equals $H$ bits, as follows

$$
\operatorname{PER}\left(j^{\prime}\right)=1-\left[1-P_{T}\left(j^{\prime}\right)\right]^{H}
$$

However, packet errors due to MAI can eventually occur in a flexible network with several different user classes transmitting simultaneously. This can result in several packets received with errors after their transmission through the optical channel. Then, the probability of receiving a packet without errors during the transmission of class- $j^{\prime}$ users, denoted packet success probability, is given by

$$
P_{C}\left(j^{\prime}\right)=\left[1-P_{T}\left(j^{\prime}\right)\right]^{H} \text {. }
$$

Subsequently, considering a packet network with two-classes, the throughput of class- $j^{\prime}$, defined as the expected number of successfully received packets per time slot, is given as [101] 


$$
\beta\left(j^{\prime}\right)=\sum_{m_{j}=0}^{\infty}\left(\sum_{m_{j^{\prime}}=1}^{\infty} m_{j^{\prime}} \cdot P_{C}\left(j^{\prime}\right) \cdot f\left(m_{j^{\prime}}\right)\right) f\left(m_{j}\right)
$$

where $f\left(m_{j^{\prime}}\right)$ is the steady-state probability distribution of composite packets arrivals. The composite packets arrival distribution of the desired user class- $j^{\prime}$ might be Poissonian when considered an infinite number of users in the network [58]. Accordingly, this probability can be expressed as

$$
f\left(m_{j^{\prime}}\right)=\frac{G_{j^{\prime}}^{m_{j^{\prime}}}}{m_{j^{\prime}} !} e^{-G_{j^{\prime}}},
$$

where $m_{j^{\prime}} \in\left\{0,1, \ldots, U_{j^{\prime}}\right\}$ is the number of simultaneous class- $j^{\prime}$ transmitting users, and $G_{j^{\prime}}$ is the offered load of class- $j^{\prime}$. Accordingly, the composite packets arrival distribution of class- $j$ is given as

$$
f\left(m_{j}\right)=\frac{G_{j}^{m_{j}}}{m_{j} !} e^{-G_{j}}
$$

where $m_{j} \in\left\{0,1, \ldots, U_{j}\right\}$ is the number of simultaneous class- $j$ s transmitting users, and $G_{j}$ is the offered load of class- $j$.

\subsection{Packet Throughput}

In this subsection, we initially introduce and describe important features of the flexible OCDMA network that are used afterwards in the throughput derivation. Then, we derive new and general mathematical expressions that can be used to evaluate the packet throughput performance considering any number of simultaneous user classes in the network. Furthermore, we derive the throughput expressions considering Poisson, binomial, and Markov chain approaches for the composite packets arrival distributions, where the latter is defined as benchmark against which the throughput performance can be compared. The mathematical formalisms developed here can be successfully applied to either 1-D or 2-D codes. They can be further applied to other modulation formats (such as phase-shift keying) as long as the new BER is provided. To get a good insight into the derivations, we firstly present and describe important aspects of the flexible packet OCDMA network so that we can present the derivations in the next subsections. 
Let us consider a flexible OCDMA network arranged in a star topology in which the users are separated into classes according to their service demands (QoS and transmission rate) and connected to the multiple access channel via optical fibers as illustrated in Figure 3.3. This time-slotted network consists of $J$-class users that share the same optical medium. The input power at the passive star coupler is split and transmitted equally among all $J$-class users. Normally, in a slotted packet network the time is divided into equal slots, where each user transmits at the beginning of a slot [58], [102]. Without loss of generality, deleterious sources such as channel impairments and time jitter are neglected since the focus is on the MAI between the users' codes. A unique optical code sequence is assigned to each user, where each user transmits one packet through the network in a single time slot. The users are divided into classes according to their required QoS and transmission rates. In the analysis, the following assumptions are made: 1) different users' classes coexist in the same time slot, where packet transmission errors can occur due to MAI; 2) the transmitter can independently determine the success of a packet transmission, and schedule a packet for retransmission when it is received with errors; 3) the overhead required by error detection techniques at the receiver is neglected for the sake of simplicity [58]. In addition, the slotted ALOHA (S-ALOHA) protocol is employed to provide medium access in the media access control (MAC) layer [58], [103] and the network transmits on-off pulses employing on-off keying (OOK) modulation. Briefly, S-ALOHA is a multiple access protocol for coordinating how multiple users access a common communication channel without interference or collision.

In the multiservice multirate J-class OCDMA slotted network, for each user class- $j \in$ $\{1,2, \ldots, J\}$ the users' data rate is defined according with its given code length in a manner that high rate users have smaller code length and low rate users have longer code length such that $L_{1}<L_{2}<\cdots<L_{j}<\cdots<L_{J}$, and $L_{j}=T_{j} / T_{c}$, where $T_{j}$ and $T_{c}$ are the bit and chip period, respectively [39]. $T_{c}$ is assumed constant and the same for all classes $J$, and the transmission power of all users $U$ in all classes $J$ is normalized to unity.

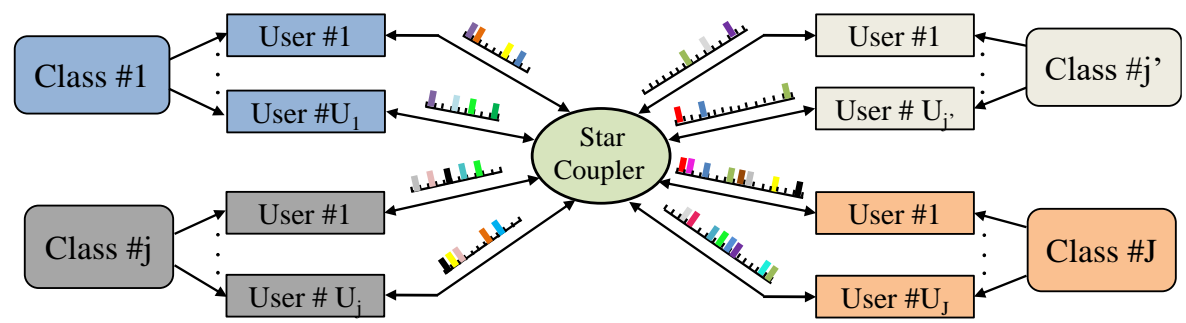

Figure 3.3: Block diagram of the flexible OCDMA packet network with users arranged into classes in accordance with their QoS and rate transmission requirements. Each user class has its own code's parameters specifications. The codes' pulses are colourless in the 1-D network case. 
In addition, the network supports multiservice (differentiated services, or equivalently QoS) transmission through specific code weights $W_{j}$ in a manner that the larger the code weight, the higher the QoS supported. Moreover, without any loss of generality, it is assumed that the desired user is the first user in the desired class denoted as $j^{\prime}$ [95]. The total number of users in the network is $\mathrm{U}=\sum_{j=1}^{J} U_{j}$, where $U_{j}$ is the number of class- $j$ users. The bit transmission rate and the packet length of class- $j$ is defined, respectively, as $R_{j}=1 / T_{j}$ and $H_{j}$. Then, the packet period of class- $j$ becomes

$$
T_{p_{j}}=\frac{H_{j}}{R_{j}}=T_{p} .
$$

Similarly, the bit transmission rate and the packet length of class- $j^{\prime}$ is defined as $R_{j^{\prime}}=$ $1 / T_{j^{\prime}}$ and $H_{j^{\prime}}$, respectively, resulting in the following expression for the packet period of class- $j^{\prime}$

$$
T_{p_{j^{\prime}}}=\frac{H_{j^{\prime}}}{R_{j^{\prime}}}=T_{p} .
$$

It can be clearly noted from (3.28) and (3.29) that the packet period of both classes is the same (see also Figure 3.4). It is worth pointing out that each class has a different $T_{j}$ and, consequently, a different $R_{j}$. Then a viable way of keeping the packet period $\left(T_{p}\right)$ fixed is by allocating a different number of bits according to each specific class so as to guarantee that $T_{p}$ is the same for all classes. Therefore, in a multirate network the number of bits transmitted in each packet class can be different from each other. Assuming $H_{J}$ as the number of bits in class- $J$, then the number of bits in class- $j$ can be simply calculated as [39]

$$
H_{j}=\left\lfloor\frac{L_{J}}{L_{j}} H_{J}\right\rfloor,
$$

where $[$.$] denotes the integer part. Independently of the number of classes present in$ the network, (3.30) allows one to calculate the number of bits in all classes.

An example of a three-class network, namely class- 1 with $L_{1}=4$ (class- $j$ ), class- 2 with $L_{2}=5$ (class- $\left.j+1\right)$, and class- 3 with $L_{3}=8$ and $H_{3}=2$ bits (class- $J$ ) is provided in Figure 3.4. It can be noted that a packet from class-1has twice the number of bits than a packet of class-3 $\left(H_{j^{\prime}}=H_{1}=4\right.$, refer to (3.30)). Notice further that the bit period of class-3 ( $T_{j=3}$, and consequently $\left.L_{3}\right)$ is twice as long as the bit period of class- $1\left(T_{j=1}\right.$, and consequently $\left.L_{1}\right)$. 

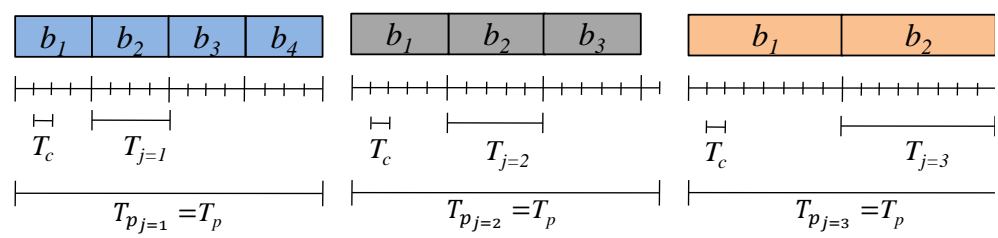

Figure 3.4: Example of the S-ALOHA OCDMA packet model for a three-class multirate network, where class- 1 ( $L_{1}=4$ and $H_{1}=4$ bits), class-2 ( $L_{2}=5$ and $H_{2}=3$ bits), and class-3 ( $L_{3}=8$ and $H_{3}=2$ bits) have the same packet length, $T_{p 1}=T_{p 2}=T_{p 3}=T_{p}$. Picture taken from [39].

Accordingly, class-3 transmits half the number of bits of class- 1 while keeping the same packet period $\left(T_{p}\right)$ for both classes [104], [105]. On the other hand, class-2 transmits one bit more than does class-3 $\left(H_{j}=H_{2}=3\right.$, refer to (3.30)) because $T_{j=2}$ (and consequently $L_{2}$ ) is smaller than $T_{j=3}$ (and consequently $L_{3}$ ) while keeping the same packet period $\left(T_{p}\right)$.

Next, Figure 3.5 illustrates the packet flow. Initially, the system is empty and users generate new packets. After their transmission over the optical channel, some packets might be received with errors due to MAI. Then, these packets are retransmitted after a random delay. In the next time slot, the arrivals are composed of both new and retransmitted packets [102], [103].

As previously mentioned, several packets might be received incorrectly with bit errors due to MAI, then the complement of the packet correct probability, denoted packet error probability, with $m$ simultaneous packet transmissions from all users' classes in the channel can be defined as $P_{E}\left(j^{\prime}\right)=1-P_{C}\left(j^{\prime}\right)$.

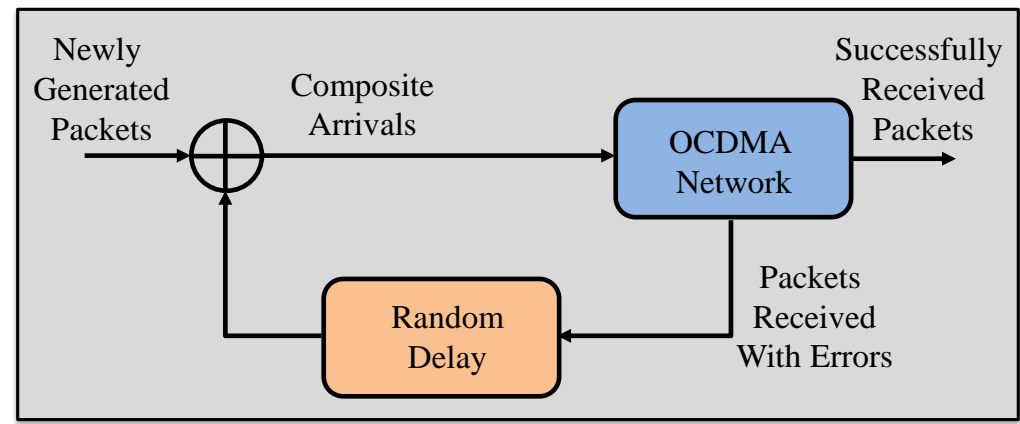

Figure 3.5: Packet flow model of the S-ALOHA OCDMA network. Picture adapted from [102]. 
Next, the probability of receiving a packet without errors during the transmission of class- $j^{\prime}$ users, denoted as packet correct probability, assuming $H_{j^{\prime}}$ as the length of class- $j^{\prime}$ packet expressed in bits, can be written as

$$
P_{C}\left(j^{\prime}\right)=\left[1-B E R\left(j^{\prime}\right)\right]^{H_{j^{\prime}}},
$$

\subsubsection{Poisson Approach}

In this subsection, we proceed with the analytical model of the flexible packet OCDMA network and introduce the mathematical formalism for throughput performance evaluation based on the Poisson distribution for the composite packet arrivals.

Let us consider a random variable $M$ representing the number of total simultaneous packets transmissions in a time slot. Also, consider that errors in different packets can occur independently of one another. Thus, the conditional distribution of successfully received packets $S$ becomes [102]

$$
P(S=s \mid M=m)=\left(\begin{array}{c}
m \\
S
\end{array}\right) P_{C}^{s}\left(j^{\prime}\right) P_{E}^{m-s}\left(j^{\prime}\right)
$$

The steady-state network throughput $\beta$, defined as the expected number of successful packets transmissions per time slot, can be expressed as

$$
\begin{aligned}
\beta\left(j^{\prime}\right) & =\mathrm{E}[\mathrm{S}]=\mathrm{E}\{\mathrm{E}[\mathrm{S} \mid M]\} \\
& =E\left[\sum_{s=0}^{M} s\left(\begin{array}{c}
M \\
s
\end{array}\right) P_{C}{ }^{s}\left(j^{\prime}\right) P_{E}{ }^{M-s}\left(j^{\prime}\right)\right] .
\end{aligned}
$$

The summations in (3.33) can be rearranged as follows

$$
\sum_{s=0}^{M} s\left(\begin{array}{c}
M \\
s
\end{array}\right) P_{C}{ }^{s}\left(j^{\prime}\right) P_{E}{ }^{M-s}\left(j^{\prime}\right)=\sum_{s=1}^{M} s\left(\begin{array}{c}
M \\
s
\end{array}\right) P_{C}{ }^{s}\left(j^{\prime}\right) P_{E}{ }^{M-s}\left(j^{\prime}\right)
$$

where $s\left(\begin{array}{c}M \\ s\end{array}\right)$ can be easily demonstrated to be equal to $M\left(\begin{array}{c}M-1 \\ s-1\end{array}\right)$. Then, (3.34) can be cast as follows 


$$
\begin{aligned}
\sum_{s=1}^{M} s\left(\begin{array}{c}
M \\
s
\end{array}\right) P_{C}{ }^{s}\left(j^{\prime}\right) P_{E}{ }^{M-s}\left(j^{\prime}\right) & =\sum_{s=1}^{M} M\left(\begin{array}{c}
M-1 \\
s-1
\end{array}\right) P_{C}{ }^{s}\left(j^{\prime}\right) P_{E}{ }^{M-s}\left(j^{\prime}\right) \\
& =M P_{C}\left(j^{\prime}\right) \sum_{s=1}^{M}\left(\begin{array}{c}
M-1 \\
s-1
\end{array}\right) P_{C}{ }^{s-1}\left(j^{\prime}\right) P_{E}{ }^{M-s}\left(j^{\prime}\right) \\
& =M P_{C}\left(j^{\prime}\right) \sum_{i=0}^{M-1}\left(\begin{array}{c}
M-1 \\
i
\end{array}\right) P_{C}{ }^{i}\left(j^{\prime}\right) P_{E}{ }^{M-1-i}\left(j^{\prime}\right) \\
& =M P_{C}\left(j^{\prime}\right)\left[P_{C}\left(j^{\prime}\right)+P_{E}\left(j^{\prime}\right)\right]^{M-1} \\
& =M P_{C}\left(j^{\prime}\right) .
\end{aligned}
$$

Finally, after substituting (3.35) into (3.33), the general network throughput in packets per slot of class- $j^{\prime}$ can be rewritten as

$$
\begin{aligned}
\beta\left(j^{\prime}\right) & =E\left[M P_{C}\left(j^{\prime}\right)\right] \\
& =\sum_{m=1}^{\infty} m P_{C}\left(j^{\prime}\right) f_{M}(m),
\end{aligned}
$$

where $f_{M}(m)$ is the general steady-state probability distribution of composite arrivals (new and retransmitted packets) [102].

It is worth to point out that (3.36) allows the evaluation of the network throughput for the three approaches considered here for composite arrival distributions $f_{M}(m)$. To calculate $f_{M}(m)$ it is assumed a packet flow model with two modes of operation, namely, origination and backlogged mode [102]. Users in the origination mode transmit new packets in a time slot with probability $p_{o}$. When these new packets' transmissions fail, the affected users enter in backlogged mode and then try to retransmit the packets after a random delay with probability $p_{r}$.

Initially, the composite arrival distribution of class-j' packets can be considered Poissonian when $p_{o}=p_{r} \rightarrow 0$ and $U \rightarrow \infty$ [102]. Therefore, the steady-state Poisson probability distribution of class- $j^{\prime}$ composite arrivals, $f_{P O I}\left(m_{j^{\prime}}\right)$, is given by

$$
f_{M}(m)=f_{P O I}\left(m_{j^{\prime}}\right)=\frac{G_{j^{\prime}}^{m_{j^{\prime}}}}{m_{j^{\prime}} !} e^{-G_{j^{\prime}}},
$$

where $m_{j^{\prime}} \in\left\{0,1, \ldots, U_{j^{\prime}}\right\}$ is the number of simultaneous class- $j^{\prime}$ transmitting users, and $G_{j^{\prime}}$ is the offered load of class- $j^{\prime}$ defined as the average number of generated 
packets from class $-j$. Accordingly, the Poisson probability distribution of class $-j$ composite arrivals, $f_{P O I}\left(m_{j}\right)$ is given as

$$
f_{M}(m)=f_{P O I}\left(m_{j}\right)=\frac{G_{j}^{m_{j}}}{m_{j} !} e^{-G_{j}},
$$

where $m_{j} \in\left\{0,1, \ldots, U_{j}\right\}$ is the number of simultaneous class- $j$ transmitting users, and $G_{j}$ is the offered load of class- $j$. With the help of (3.36) and (3.37), the throughput of class- $j^{\prime}$ can be obtained as

$$
\beta_{P O I}^{P}\left(j^{\prime}\right)=\sum_{m_{j^{\prime}}=1}^{\infty} m_{j^{\prime}} P_{C}\left(j^{\prime}\right) f_{P O I}\left(m_{j^{\prime}}\right) .
$$

Furthermore, for a two-class multiservice network [105], where for example the desired class- $j^{\prime}$ is the first class and the interfering class is the second one, the throughput becomes

$$
\begin{aligned}
\beta_{P O I}(1) & =\sum_{m_{2}=0}^{\infty} \sum_{m_{1}=1}^{\infty} m_{1} P_{C}\left(j^{\prime}\right) f_{P O I}\left(m_{1}\right) f_{P O I}\left(m_{2}\right) \\
& =\sum_{m_{2}=0}^{\infty} \sum_{m_{1}=1}^{\infty} m_{1} P_{C}\left(j^{\prime}\right) \prod_{j=1}^{2} f_{P O I}\left(m_{j}\right) .
\end{aligned}
$$

For a three-class multiservice network [106], where for example the desired class- $j$ ' is the first class and the interfering classes are the second and the third classes, the throughput becomes

$$
\begin{aligned}
\beta_{P O I}(1) & =\sum_{m_{3}=0}^{\infty} \sum_{m_{2}=0}^{\infty} \sum_{m_{1}=1}^{\infty} m_{1} P_{C}\left(j^{\prime}\right) f_{P O I}\left(m_{1}\right) f_{P O I}\left(m_{2}\right) f_{P O I}\left(m_{3}\right), \\
& =\sum_{m_{3}=0}^{\infty} \sum_{m_{2}=0}^{\infty} \sum_{m_{1}=1}^{\infty} m_{1} P_{C}\left(j^{\prime}\right) \prod_{j=1}^{3} f_{P O I}\left(m_{j}\right) .
\end{aligned}
$$

By way of the Poisson properties $(U \rightarrow \infty)$ as well as for convenience sake, the summation for $J$-class can be generalized as follows

$$
\sum_{m_{J}=0}^{U_{j}} \sum_{m_{J-1}=0}^{U_{j-1}} \ldots \sum_{m_{2}=0}^{U_{2}} \sum_{m_{1}=0}^{U_{1}}\{\ldots\}=\sum_{j=0}^{J} \sum_{m_{j}=0}^{U_{j}}\{\ldots\} .
$$




\section{MATHEMATICAL ForMALISMS}

Only for the sake of convenience and simplicity, the upper bound of the summations in (3.42) are kept as $U$ rather than $\infty$. Therefore, one can finally obtain the total throughput of class- $j^{\prime}, \beta_{P O I}\left(j^{\prime}\right)$, for a general number of classes, J-class, as [39]

$$
\beta_{P O I}\left(j^{\prime}\right)=\sum_{\substack{j=1 \\ j \neq j^{\prime}}}^{J} \sum_{m_{j}=0}^{\infty} \sum_{m_{j^{\prime}}=1}^{\infty} m_{j^{\prime}} P_{C}\left(j^{\prime}\right) \prod_{j=1}^{J} f_{P O I}\left(m_{j}\right) .
$$

\subsubsection{Binomial Approach}

In this subsection, we proceed with the derivation of the general packet throughput equation considering the binomial distribution for the composite packet arrivals.

Next, we consider the assumptions done in the previous subsection from equation (3.28) to (3.36) and the packet flow model. Thus, the composite arrival distribution of class $-j^{\prime}$ packets is considered binomial when $p_{o}=p_{r}$ and the number of users in the network is finite [102].

Therefore, the steady-state binomial probability distribution of class- $j^{\prime}$ composite arrivals, $f_{B I N}\left(m_{j^{\prime}}\right)$, becomes

$$
f_{M}(m)=f_{B I N}\left(m_{j^{\prime}}\right)=\left(\begin{array}{c}
U_{j^{\prime}} \\
m_{j^{\prime}}
\end{array}\right)\left(\frac{G_{j^{\prime}}}{U_{j^{\prime}}}\right)^{m_{j^{\prime}}}\left(1-\frac{G_{j^{\prime}}}{U_{j^{\prime}}}\right)^{U_{j^{\prime}}-m_{j^{\prime}}},
$$

where $U_{j^{\prime}}$ is the number of users in the desired class- $j^{\prime}$. Accordingly, the binomial probability distribution of class- $j$ composite arrivals, $f_{B I N}\left(m_{j}\right)$, is

$$
f_{M}(m)=f_{B I N}\left(m_{j}\right)=\left(\begin{array}{c}
U_{j} \\
m_{j}
\end{array}\right)\left(\frac{G_{j}}{U_{j}}\right)^{m_{j}}\left(1-\frac{G_{j}}{U_{j}}\right)^{U_{j}-m_{j}} .
$$

After substituting (3.44) into (3.36), the following expression for the throughput of class- $j^{\prime}$ is obtained

$$
\beta_{b i n}^{P}\left(j^{\prime}\right)=\sum_{m_{j^{\prime}}=1}^{U_{j^{\prime}}} m_{j^{\prime}} P_{C}\left(j^{\prime}\right) f_{B I N}\left(m_{j^{\prime}}\right)
$$


Finally, considering all classes $J$ of the multiservice multirate network and following the same steps as in the previous subsection, the total throughput of class- $j$ ' in packets per slot is expressed as follows [39]

$$
\beta_{b i n}\left(j^{\prime}\right)=\sum_{\substack{j=1 \\ j \neq j^{\prime}}}^{J} \sum_{m_{j}=0}^{U_{j}} \sum_{m_{j^{\prime}}=1}^{U_{j^{\prime}}} m_{j^{\prime}} P_{C}\left(j^{\prime}\right) \prod_{j=1}^{J} f_{B I N}\left(m_{j}\right) .
$$

\subsubsection{Markov Chain Approach}

In this subsection, the packet throughput expression is developed based on the discrete-time Markov chain. The analytical formalism developed here is based on the formalism developed for single-rate CDMA radio networks [102]. The Markov chain approach can take any arbitrary value of $p_{0}$ and $p_{r}$ with a finite number of users in the network. A Markov chain $X_{t}$ has a finite state-space $\left\{0,1,2, \ldots, n, U_{j^{\prime}}\right\}$ with a transition matrix defined as $P=\left[P n m: n, m \in U_{j^{\prime}}\right]$, where each state $n$ corresponds to the number of $n$ backlogged users. $P_{n m}$ denotes the probability that $m$ backlogged users will be present in the next state given that $n$ are present in the current state. This one-step transition probability from state $n$ to state $m$ can be written as

$$
P_{n m}=\operatorname{Pr}\left\{X_{t+1}=m \mid X_{t}=n\right\} .
$$

Notice that when leaving state $n$ the chain must move to one of the states $m$, with $m$ $\in U_{j^{\prime}}$ knowing that each row of the transition square matrix sums to one.

Next, a transition from state $n$ to state $m$ is determined by the difference between the number of unsuccessful new transmissions, denoted as U-NTX, and successful retransmissions, denoted as S-RTX. Then, the system state can change only due to unsuccessful new transmissions or successful retransmissions. In a given time slot, there are $\mathrm{NTX}=\xi_{0}$ new transmissions and $\mathrm{RTX}=\xi_{r}$ retransmissions of packets with probabilities of U-NTX and S-RTX defined, respectively, as [102]

$$
\operatorname{Pr}\left\{U-N T X=l \mid N T X=\xi_{0}, R T X=\xi_{r}\right\}=b\left[l, \xi_{0}, P_{E}\left(\xi_{0}+\xi_{r}\right)\right], \quad \xi_{0} \geq l,
$$

and

$$
\operatorname{Pr}\left\{S-R T X=k \mid N T X=\xi_{0}, R T X=\xi_{r}\right\}=b\left[k, \xi_{r}, P_{C}\left(\xi_{0}+\xi_{r}\right)\right], \quad \xi_{r} \geq k,
$$




\section{MATHEMATICAL FORMALISMS}

where $b(\delta, \alpha, p)=\left(\begin{array}{l}\alpha \\ \delta\end{array}\right) p^{\delta}(1-p)^{\alpha-\delta}$ is the probability mass function of the binomial distribution. The probability of $\xi_{0}$ attempted new transmissions and $\xi_{r}$ attempted retransmissions, where $\xi_{0}$ and $\xi_{r}$ are independent Bernoulli random variables, given that $n$ backlogged users are present in the current state are, respectively, $b\left(\xi_{0}, U_{j^{\prime}}-\right.$ $\left.n, p_{0}\right)$ and $b\left(\xi_{r}, n, p_{r}\right)$. Therefore, employing these definitions, (3.49), and (3.50), the joint probability distribution of $U-N T X$ and $S-R T X$ become

$$
\begin{gathered}
\operatorname{Pr}\left\{S-R T X=k, U-N T X=l \mid X_{t}=n\right\}= \\
=\sum_{\xi_{0}=l}^{U_{j^{\prime}-n}} \sum_{\xi_{r}=k}^{n} b\left[l, \xi_{0}, P_{E}\left(\xi_{0}+\xi_{r}\right)\right] \cdot b\left[k, \xi_{r}, P_{C}\left(\xi_{0}+\xi_{r}\right)\right] \cdot b\left(\xi_{0}, U_{j^{\prime}}-n, p_{0}\right) \cdot b\left(\xi_{r}, n, p_{r}\right) .
\end{gathered}
$$

It is noteworthy that when $m \geq n$ a transition from state $n$ to $m$ occurs if $U$-NTX exceeds $S-R T X$ by $(m-n)$. Conversely, when $m \leq n$ a transition occurs if $S-R T X$ exceeds $U$ NTX by $(n-m)$. Thus, the one-step state transition probabilities of the Markov chain $X_{t}$ can be expressed as [102]

$$
P_{n m}= \begin{cases}\sum_{v=0}^{\min \left(n, U_{j^{\prime}}-m\right)} \operatorname{Pr}\left\{S-R T X=v, U-N T X=m-n+v \mid X_{t}=n\right\}, & m \geq n \\ \sum_{v=0} \operatorname{Pr}\left\{S-R T X=n-m+v, U-N T X=v \mid X_{t}=n\right\}, & m \leq n .\end{cases}
$$

The finite state-space Markov chain $X_{t}$ is irreducible and aperiodic with a transition matrix $P$, which implies the existence of a long-term state occupancy probability distribution $\mu$ [107], [103]. This probability distribution vector $\mu$ can be computed by solving the following linear equation system, with normalization constraint $\sum_{n=0}^{U_{j^{\prime}}} \mu(n)=1$,

$$
\mu^{T}=\mu^{T} P
$$

where $\mu^{T}=\left[\mu(0), \mu(1), \ldots, \mu\left(U_{j^{\prime}}\right)\right]$.

After calculating the matrix of transition $P$ given by (3.52), it is desired to solve (3.53). Generally, if the matrix is a $U_{j^{\prime}} \times U_{j^{\prime}}$ square matrix, it has $U_{j^{\prime}}$ eigenvalues and eigenvectors. A neat approach to calculate (3.53) is to firstly compute the eigenvalues and eigenvectors of the transpose of $P$, then to find the index of the eigenvalue element equal to its largest entry. Next, the eigenvector matched to this same index is normalized so that its summation adds to 1 . Finally, after this procedure, the desired eigenvector is the transpose of the normalized eigenvector. 
Next, the steady-state composite arrival distribution for $n$ backlogged users in the current time slot, $X_{t}=n$, is given by

$$
\begin{aligned}
f_{M}(m \mid n) & =\sum_{\substack{v=\max (m-n, 0) \\
\min \left(m, U_{j^{\prime}}-n\right)}}^{\min \left(m, U_{j^{\prime}}-n\right)} \operatorname{Pr}\{N T X=v, T R X=m-v\} \\
& =\sum_{v=\max (m-n, 0)} b\left(v, U_{j^{\prime}}-n, p_{0}\right) \cdot b\left(m-v, n, p_{r}\right) .
\end{aligned}
$$

Therefore, the steady-state packet throughput based on Markov chain approach can be found employing (3.23), the normalization $\sum_{n=0}^{U_{j^{\prime}}} \mu(n)=1$, the solution of (3.53), and (3.54), which gives

$$
\begin{aligned}
\beta_{m a r}\left(j^{\prime}\right) & =\sum_{\substack{m=1 \\
U_{j^{\prime}}}}^{U_{j^{\prime}}} m P_{C}\left(j^{\prime}\right) 1 f_{M}(m)=\sum_{m=1}^{U_{j^{\prime}}} m P_{C}\left(j^{\prime}\right) \sum_{n=0}^{U_{j^{\prime}}} \mu(n) f_{M}(m) \\
& =\sum_{m=1}^{U_{j^{\prime}}} m P_{C}\left(j^{\prime}\right)\left\{\sum_{n=0} \mu(n) f_{M}(m \mid n)\right\} .
\end{aligned}
$$

Finally, the offered load of class- $j^{\prime}$ in packets per slot, when $p_{o}=p_{r}=p$, is given as $G_{j^{\prime}}=U_{j^{\prime}} p$.

\subsubsection{Packet Throughput for Multicode Technique}

This subsection addresses the mathematical formalism developed for the multicode technique along with a new packet throughput equation for performance evaluation.

In an OCDMA network that supports multirate transmissions using multicode technique, a set of codes is assigned to each user so that the user can achieve arbitrary multirate transmissions. Moreover, the several codes assigned to a single user have the same length. Further details on this technique can be found in Section 2.5. For each user class $j \in\{1,2, \ldots, J\}$ the users' data rate is defined according to the number of $B_{j}$ assigned codes (times the network basic bit rate), i.e., a large number of codes is assigned to high rate users while a single code is assigned to low rate users. Then, a high data rate user transmits at $B_{j}$ times the network basic bit rate [87], [108]. The bit 


\section{MATHEMATICAL FORMALISMS}

transmission rate and the compound packet length of class- $j$ for a network using multicode technique are defined, respectively, as $B_{j} \cdot \mathcal{R}_{j}$ and $B_{j} \cdot \mathcal{H}_{j}$. Then, the packet period of class- $j$ will be

$$
\Gamma_{p_{j}}=\frac{B_{j} \cdot \mathcal{H}_{j}}{B_{j} \cdot \mathcal{R}_{j}}=\frac{\mathcal{H}_{j}}{\mathcal{R}_{j}}=T_{p}
$$

Bit transmission rate and packet length of class- $j^{\prime}$ are defined as $B_{j^{\prime}} \cdot \mathcal{R}_{j^{\prime}}$ and $B_{j^{\prime}} \cdot \mathcal{H}_{j^{\prime}}$ respectively, resulting in the following expression for the packet period of class- $j^{\prime}$

$$
\Gamma_{p_{j^{\prime}}}=\frac{B_{j^{\prime}} \cdot \mathcal{H}_{j^{\prime}}}{B_{j^{\prime}} \cdot \mathcal{R}_{j^{\prime}}}=\frac{\mathcal{H}_{j^{\prime}}}{\mathcal{R}_{j^{\prime}}}=T_{p}
$$

Thus, the packet period of both classes is the same for the multicode technique. It is noteworthy that in this technique all classes have the same bit period. Thus, the number of bits in all classes is the same and is given by $\mathcal{H}_{j^{\prime}}$.

This technique eventually requires a code family with large cardinality so that several high rate users can be accommodated in the network. In this way, amongst several codes proposed for OCDMA networks, the 2-D multi-weight multi-length optical orthogonal code (MWML-OOC) [54] is considered a good choice due to its simple construction algorithm and very large cardinality. Accordingly, the overall cardinality of the 2-D MWML-OOC is given as [54]

$$
C=\phi_{\text {ooC }} P^{2}=\sum_{j=1}^{J} K_{j} P^{2},
$$

where $P$ is a prime number representing the number of available wavelengths employed in the code construction [54], and $K_{j}$ is the code cardinality (equals to the number of users) in class- $j$, which is bounded by

$$
\frac{\sum_{j=1}^{J} K_{j} W_{j}\left(W_{j}-1\right)}{L_{J}-1} \leq 1 .
$$

The general network throughput in packets per slot of class-j' can be rewritten as

$$
\begin{aligned}
\beta\left(j^{\prime}\right) & =E\left[M P_{C}\left(j^{\prime}\right)\right] \\
& =\sum_{m=1}^{\infty} B_{j^{\prime}} m P_{C}\left(j^{\prime}\right) f_{M}(m),
\end{aligned}
$$


where $B_{j^{\prime}}$ is the multicode factor, and $f_{M}(m)$ is the general steady-state probability distribution of composite arrivals (new and retransmitted packets) [106]. In the calculation of $f_{M}(m)$ it is assumed a packet flow model with two modes of operation, namely, origination and backlogged mode.

The composite arrival distribution of class- $j^{\prime}$ packets is considered Poissonian when $p_{o}=p_{r} \rightarrow 0$ and $U \rightarrow \infty$ [102]. Therefore, the steady-state Poisson probability distribution of class- $j^{\prime}$ composite arrivals, $f_{P O I}\left(m_{j^{\prime}}\right)$, is given by [94]

$$
f_{M}(m)=f_{P O I}\left(m_{j^{\prime}}\right)=\frac{\left(G_{j^{\prime}} / B_{j^{\prime}}\right)^{m_{j^{\prime}}}}{m_{j^{\prime}} !} e^{-G_{j^{\prime}} / B_{j^{\prime}}},
$$

where $m_{j^{\prime}} \in\left\{0,1, \ldots, U_{j^{\prime}}\right\}$ is the number of simultaneous class- $j^{\prime}$ transmitting users, and $G_{j^{\prime}}$ is the offered load of class- $j^{\prime}$ defined as the average number of generated packets from class- $j^{\prime}$. Accordingly, the Poisson probability distribution of class $-j$ composite arrivals, $f_{P O I}\left(m_{j}\right)$, is given as

$$
f_{M}(m)=f_{P O I}\left(m_{j}\right)=\frac{\left(G_{j} / B_{j}\right)^{m_{j}}}{m_{j} !} e^{-G_{j} / B_{j}},
$$

where $m_{j} \in\left\{0,1, \ldots, U_{j}\right\}$ is the number of simultaneous class $-j$ transmitting users, and $G_{j}$ is the offered load of class-j. Subsequently, employing (3.60) and (3.62), the throughput of class- $j^{\prime}$ can be obtained as

$$
\beta_{P O I}^{P}\left(j^{\prime}\right)=\sum_{m_{j^{\prime}}=1}^{\infty} B_{j^{\prime}} m_{j^{\prime}} P_{C}\left(j^{\prime}\right) f_{P O I}\left(m_{j^{\prime}}\right) .
$$

Furthermore, for a two-class multiservice network, where for example the desired class $-j$ ' is the first class and the interfering class is the second one, the throughput will be

$$
\begin{aligned}
\beta_{P O I}(1) & =\sum_{m_{2}=0}^{\infty} \sum_{m_{1}=1}^{\infty} B_{1} m_{1} P_{C}\left(j^{\prime}\right) f_{P O I}\left(m_{1}\right) f_{P O I}\left(m_{2}\right) \\
& =\sum_{m_{2}=0}^{\infty} \sum_{m_{1}=1}^{\infty} B_{1} m_{1} P_{C}\left(j^{\prime}\right) \prod_{j=1}^{2} f_{P O I}\left(m_{j}\right) .
\end{aligned}
$$

From the Poisson properties $(U \rightarrow \infty)$ and for convenience sake, the summation for $J$ class can be generalized as follows 


$$
\sum_{m_{J}=0}^{\infty} \sum_{m_{J-1}=0}^{\infty} \ldots \sum_{m_{2}=0}^{\infty} \sum_{m_{1}=0}^{\infty}\{\ldots\}=\sum_{j=0}^{J} \sum_{m_{j}=0}^{\infty}\{\ldots\}
$$

Therefore, one can finally obtain the total throughput of class- $j^{\prime}$ in packets per slot, $\beta_{P O I}\left(j^{\prime}\right)$, for a general number of classes, J-class, as [109]

$$
\beta_{P O I}\left(j^{\prime}\right)=\sum_{\substack{j=1 \\ j \neq j^{\prime}}}^{J} \sum_{m_{j}=0}^{\infty} \sum_{m_{j^{\prime}}=1}^{\infty} B_{j^{\prime}} m_{j^{\prime}} P_{C}\left(j^{\prime}\right) \prod_{j=1}^{J} f_{P O I}\left(m_{j}\right),
$$

where $P_{C}\left(j^{\prime}\right)$ is the packet correct probability defined as $P_{C}\left(j^{\prime}\right)=\left[1-B E R\left(j^{\prime}\right)\right]^{H_{j^{\prime}}}$.

Subsequently, considering chip-synchronous transmissions among users, decision threshold $\mu$, and data bits transmissions with equal probability $1 / 2$, the BER of class $-j^{\prime}$ users is expressed as [109]

$$
\begin{aligned}
\operatorname{BER}\left(j^{\prime}\right) & =P\left(Z \geq \mu \mid b_{1, j^{\prime}}=0\right) \cdot P\left(b_{1, j^{\prime}}=0\right)+P\left(Z<\mu \mid b_{1, j^{\prime}}=1\right) \cdot P\left(b_{1, j^{\prime}}=1\right) \\
& =\frac{1}{2} P\left(Z \geq \mu \mid b_{1, j^{\prime}}=0\right) \\
& =\frac{1}{2} \sum_{i=\mu}^{U-1}\left(\begin{array}{c}
U C-1 \\
i
\end{array}\right) \mathcal{P}^{i}(1-\mathcal{P})^{U-1-i}
\end{aligned}
$$

where $U C$ is the total number of active codes in the network [94] given by $U C=$ $\sum_{j=1}^{J} B_{j} U_{j}$, where $B_{j}$ is the number of class- $j$ codes assigned to each user and $\mathcal{P}$ is the multirate probability of interference given as $\mathcal{P} \approx \sum_{j=1}^{J} \frac{B_{j} N_{j} p_{j j^{\prime}}}{(U C-1)}$.

\subsection{Packet Delay}

In this section, we derive the packet delay of flexible OCDMA networks considering the three different packet throughput expressions obtained in the previous subsections.

First, it is necessary to calculate the packet throughput for each distribution (Poisson (3.43), binomial (3.47) and Markov chain (3.55)) since the average packet delay is expressed as the ratio between the offered load and packet throughput [110]. Once the packet throughput is obtained, the delay of the flexible OCDMA network for Jclass under Poisson or binomial approaches is obtained follows 


$$
D\left(j^{\prime}\right)=\frac{G_{j^{\prime}}}{\beta\left(j^{\prime}\right)},
$$

where $\beta\left(j^{\prime}\right)$ is the packet throughput under the Poisson or binomial distribution. Evidently, when $\beta\left(j^{\prime}\right)$ is used under the Poisson distribution, the delay $D\left(j^{\prime}\right)$ is also poissonian. By its turn, the general offered load of class- $j^{\prime}$ under the Markov chain approach in packets per slot is written as

$$
G_{j^{\prime}}^{G M}=\left(U_{j^{\prime}}-\eta\right) p_{o}+\eta \cdot p_{r}
$$

where $\eta=\sum_{\eta=0}^{U_{j^{\prime}}} \eta \cdot \mu(\eta)$. However, in case the general offered load of class $-j^{\prime}$ is reduced (assuming $p_{o}=p_{r}=p$ ) it becomes

$$
G_{j^{\prime}}^{M}=U_{j^{\prime}} p
$$

Therefore, the delay of flexible OCDMA networks for the J-class under the Markov chain approach can be written as

$$
D_{M}\left(j^{\prime}\right)=\frac{G_{j^{\prime}}^{M}}{\beta\left(j^{\prime}\right)},
$$

where $\beta\left(j^{\prime}\right)$ is the throughput expression obtained with the Markov Chain approach. It is worth pointing out that the general offered load allows one to fairly evaluate and compare the delay performance among the three possible approaches, Poisson, binomial and Markov chain. 



\section{Chapter 4 \\ Numerical Results and Discussions}

In this chapter, numerical simulation results addressing the performance of flexible OCDMA networks are shown and discussed. The software Matlab was used for the simulations. The proposed mathematical formalisms, described in Chapter 3, are used to assess the network performance of several scenarios. Before proceeding to the network analysis itself, a validation procedure of the proposed BER and packet throughput analytical formalisms is carried out, where the convergence of multirate OCDMA networks towards conventional single rate networks is assumed as the validation criterion. Note that the single rate network can be regarded as a particular scenario of the multirate network when only one transmission rate is considered. Therefore, the convergence between both networks is defined here as the validation criterion. A second validation criterion, the proposed BER equation based on the binomial distribution for the MAI has its performance compared to that of the Monte Carlo method, which is widely adopted in the literature as a network benchmark.

Furthermore, the following common features and conditions are assumed for all the network scenarios investigated here: a) the MAI is the only degradation source; $b$ ) the transmissions among users' signals are chip-synchronous; c) transmitted signals are OOK modulated; d) the transmission power is normalized to all users; e) the users' signals are incoherent and the decoding, as well as bit retrieval process, are based on IM/DD; e) all the employed family of codes have maximum nonzero shift autocorrelation and cross-correlation bounded by one; f) errors can occur only when the desired user transmits data bits " 0 " and, consequently, bits " 1 " are always transmitted with success; g) the scenarios are considered "back-to-back"; and h) the users are classified into classes, where each class has an exclusive transmission rate and QoS level.

Finally, this Chapter investigates the following scenarios of flexible OCDMA networks:

1) The packet throughput performance of distinct 1-D and 2-D MWML-OOC OCDMA networks assuming Poisson, binomial and Markov chain approaches for the composite packet arrivals; 
2) the BER performance of 2-D FFH-OCDMA networks assuming separately binomial and gaussian distributions for the MAI and their performance comparison;

3) the BER and packet throughput performance of 2-D MWML-OOC OCDMA networks employing the multicode technique and assuming a Poisson distribution for the composite packet arrivals;

4) the PER and packet throughput performance of 1-D IP OCDMA networks employing 1-D MWML-OOC and ODLs-based encoders and decoders;

5) the PER and packet throughput performance of 2-D IP OCDMA networks employing 2-D FFH-based codes and MBG encoders and decoders;

6) the packet delay performance of 2-D MWML-OOC OCDMA networks considering Poisson, binomial and Markov chain approaches for composite packet arrivals.

Part of the work presented in this chapter has been published as:

T. R. Raddo, A. L. Sanches, I. T. Monroy, and B.-H. V. Borges, "Throughput performance evaluation of multiservice, multirate OCDMA in flexible networks," IEEE Photonics Journal, vol. 8, no. 1, pp. 1-15, February 2016.

T. R. Raddo, A. L. Sanches, J. V. dos Reis Jr., and B. -H. V. Borges, “A new approach for evaluating the BER of a multirate, multiclass OFFH-CDMA system," IEEE Communications Letters, vol. 16, no. 2, February 2012.

T. R. Raddo, A. L. Sanches, I. T. Monroy, and B.-H. V. Borges, "Packet throughput performance of multiservice, multirate OCDMA in elastic networks", in Proc. IEEE International Conference on Communications (ICC) 2016, Kuala Lumpur, Malaysia.

T. R. Raddo, A. L. Sanches, I. T. Monroy, and B.-H. V. Borges, "Multirate IP traffic Transmission in flexible access networks based on optical FFH-CDMA" in Proc. IEEE International Conference on Communications (ICC) 2016, Kuala Lumpur, Malaysia.

T. R. Raddo, A. L. Sanches, J. V. dos Reis, Jr, and B. -H. V. Borges, "Multiservice, multirate IP transmission over OCDMA network," in Proc. IEEE ICTON, Cartagena, Spain, 2013. 


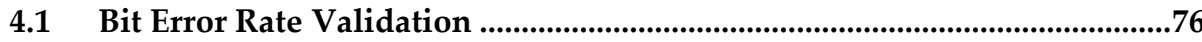

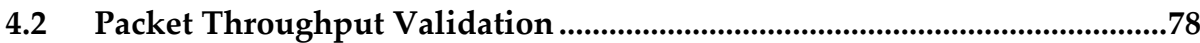

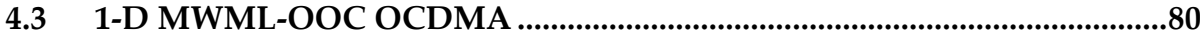

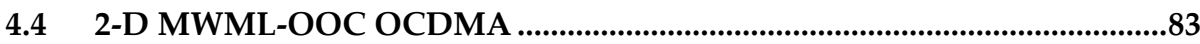

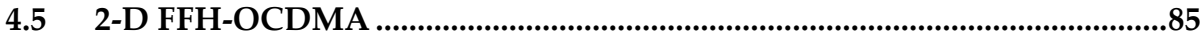

4.6 2-D MWML-OOC OCDMA, Multicode Technique .......................................90

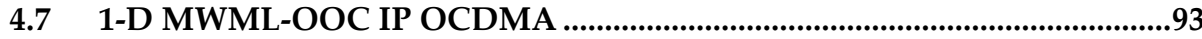

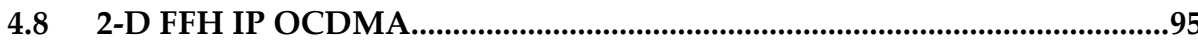

4.9 2-D MWML-OOC OCDMA, Packet Delay..................................................99 


\subsection{Bit Error Rate Validation}

In this subsection, a validation procedure of the proposed BER equation based on the binomial distribution for the MAI is carried out, where the BER convergence of multirate OCDMA networks towards that of conventional single rate networks is defined as validation criterion. In this case, the single rate network is considered a particular scenario of the multirate network with only a single transmission rate available.

Accordingly, the validation procedure consists in reproducing the performance of a conventional single rate 1-D OCDMA network applying the proposed multirate BER formalism with the network's parameters. Then, it is considered the parameters of the single rate network addressed in [1 page 243, Figure5.4(b)] with the BER formalism proposed in Subsection 3.1. The nomenclature and parameters used in this analysis are provided in Subsection 3.1. The variables' value is defined here in such a way as to obtain BER convergence from a multirate towards a single-rate network as follows: total number of classes $J=1$, the code length $L_{1}=L=1000$ code weight $W_{1}=$ $W=7$, optimum threshold $\mu=7$, and the total number of users $U_{1}=U=20$ (whereas $\left.U_{2}=0\right)$. These values are the same as used in [1]. The BER performance versus the number of simultaneous users for the conventional single rate OCDMA network is plotted in Figure 4.1, where the symbols denote the analytical formalism developed in Subsection 3.1 and the solid lines the BER using Eq. (5.17) from [1, page 242].

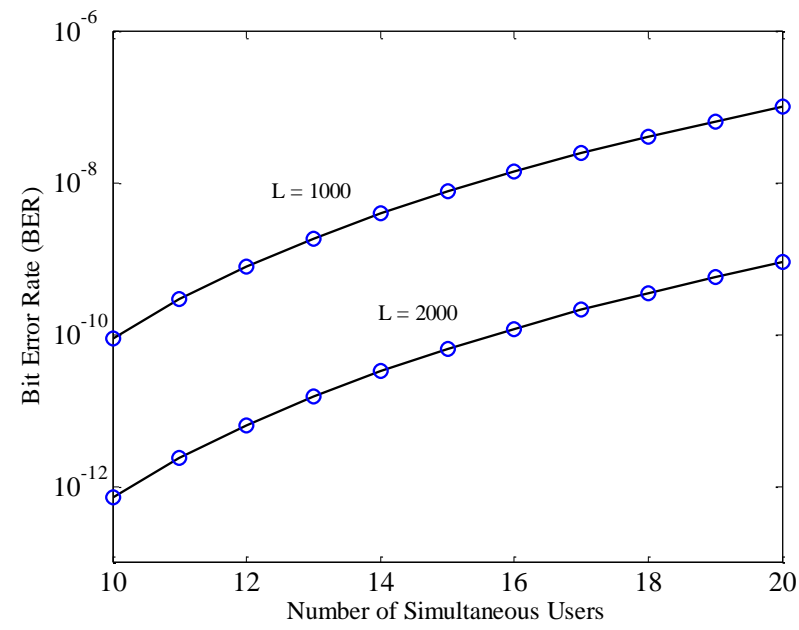

Figure 4.1. BER versus the number of simultaneous users for a conventional single-rate OCDMA network with $W=7, L=1000$ (or $L=2000$ ), and $U=20$. Multirate BER formalism considering only a single user class (symbols) and conventional single rate formalism (solid lines). 
The proposed multirate BER formalism, when set up accordingly, can reproduce the results of a single rate OCDMA network [1] with high accuracy. Observe that the multirate formalism is in excellent agreement with the BER results obtained with the single rate formalism. In this manner, it confirms that the proposed multirate formalism performs consistently with the single rate formalisms.

Next, we carry out a second BER validation procedure so that the accuracy of the proposed mathematical formalism can be further verified against now a multirate network scenario. This can be accomplished as follows. First, we assess the BER equation (3.13) by investigating the performance of a multirate OCDMA network with three-classes and compare the results with those obtained via Eq. (3.18). This is an interesting multirate scenario as it pushes the formalism to be used with different parameters and users' classes.

Then, we investigate the BER versus the number of users for three different users' classes, $J=3$, where the code length, weight, and number of users are, respectively, $L_{1}=500, W_{1}=7$, and $U_{1}=4, L_{2}=1000, W_{2}=5$, and $U_{2}=20$, and $L_{3}=1500, W_{3}=3$, and $U_{3}=20$ (the subscripts 1, 2 and 3 refer to the three different classes) [42]. Moreover, the optimum threshold value is set to the respectively user's code weight of each class. Also, the frequency parameter $F$ is set to 1 (see equation ((3.6)) since this is a 1$\mathrm{D}$ network. It is noteworthy mentioning that the users' transmission rates are transparent to the formalism and are accounted for in terms of the code length ratio between the involved classes.

The BER performance obtained with the Poisson ((3.18), solid lines) and the proposed binomial ((3.13), dashed lines) approaches are shown in Figure 4.2. The results for class-3 obtained with both formalisms closely overlap. This good agreement, which confirms that the Poisson distribution is a good approximation for this case, is due to the sparse (long) code employed in this class $\left(L_{3}=1500\right)$ and the low probability of interference among this users' class. However, it is worth mentioning that this approximation is not valid for all multirate scenarios. For example, even for the scenario considered here, the results obtained with the Poisson-based BER (black diamonds) for class- 1 users are inaccurate when compared to the ones obtained via the binomial-based BER (blue diamonds). In addition, this discrepancy can become considerably large depending on the multirate network configuration, as reported in [54] and [96].

Furthermore, the binomial-based BER results shown in Figure 4.2 agrees well with those presented in [42] using the Monte Carlo method, which is widely used in the literature as benchmark, showing again that the proposed formalism is consistent and accurate. 


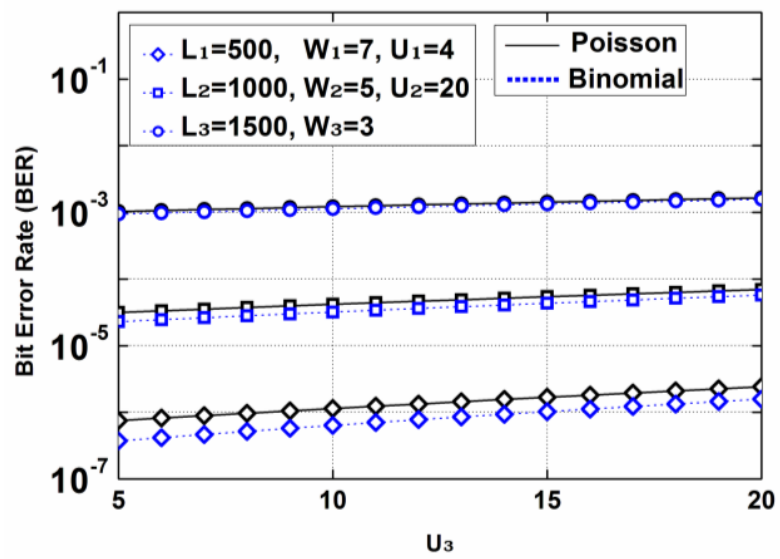

Figure 4.2: BER as a function of simultaneous users for a three classes MWML-OOC system. The MAI distributions are Poisson (solid lines) [42] and binomial (dotted lines) [96]. The subscripts 1, 2 and 3 refer to the different classes investigated.

Notice from Figure 4.2 that class-3 shows the poorest BER performance of all three classes, which is due to its lowest code weight. In addition, increasing the number of users in class-3 does not noticeably modify the overall performance of the network, since this class has very low probability of interference due to its low code weight as well as large code length. The results also show that the overall performance of this multirate network is poor, and none of the classes can transmit even under a standard BER level (BER $<10^{-9}$ ). For example, class-3 (low transmission rate) produces a BER on the order of $1 \times 10^{-3}$ (circles), while class-2 (medium transmission rate) produces a BER of about $4.5 \times 10^{-5}$.

\subsection{Packet Throughput Validation}

In this subsection, a validation procedure of the proposed packet throughput equation based on the binomial distribution for the packet composite arrivals is carried out. Again, the validation criterion is the packet throughput convergence of the multirate OCDMA network formalism (limited to one transmission rate) towards that of a conventional single rate network. This allows us to compare both approaches under similar conditions. The throughput performance of a conventional single rate OCDMA network is addressed considering only the parameters of class- 1 of a twoclass multirate OCDMA network. The three approaches adopted here for throughput evaluation are: binomial, Poisson and Markov Chain. For convenience's sake, only a well-known network scenario is adopted in this analysis [58]. Hence, let us consider a two-class OCDMA network employing 1-D MWML-OOC [42] with code length and 
weight, number of users, and packet length parameters, respective to each class, as given by: $L_{1}=600, W_{1}=4, U_{1}=36, H_{1}=1024$ bits; and $L_{2}=1200, W_{2}=5, U_{2}=0, H_{2}=512$ bits. Class-1 has low-QoS and high-transmission rate while no one from class-2 transmits, which renders a single-rate network scenario.

The BER convergence of the proposed multirate throughput formalisms towards that of the single rate formalisms can be guaranteed as long as the same set of parameters is adopted for the single rate network namely, $L=L_{1}=600, W=W_{1}=4, U=U_{1}=36$, and $H=H_{1}=1024$ bits. The throughput of the single rate OCDMA network versus the offered load based on the Poisson (dotted) [58] and binomial (dashed) [111] composite arrival distributions are plotted in Figure 4.3. Also plotted in this figure are the throughput performances obtained with the proposed formalisms Poisson ((3.43), triangles), binomial ((3.47), squares), and Markov ((3.55), circles) approaches when only a single class (class-1) is present in the network. Notice that the results obtained via the proposed throughput formalisms are in excellent agreement with those obtained with the mathematical formalism of single rate OCDMA networks.

In addition, the binomial-based results are more accurate than the Poisson-based ones when the offered load is sufficiently large (> 20 packets/slot). Therefore, this validation procedure confirms that the proposed multirate formalisms are indeed reliable and accurate. With the validation procedure complete, we now move to the analysis of more realistic network scenarios in the next section.

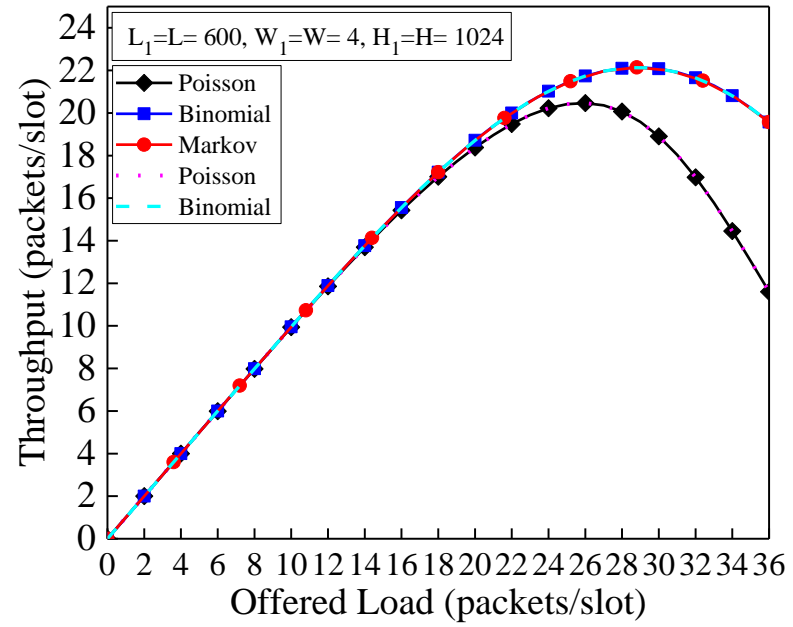

Figure 4.3: Packet throughput versus the offered load of a conventional single-rate single-service OCDMA network for validation purposes. 


\subsection{1-D MWML-OOC OCDMA}

The mathematical formalisms developed in the previous section are now applied to address the packet throughput performance of multiservice, multirate OCDMA networks. The three proposed approaches (binomial, Poisson and Markov Chain) are assessed and compared under different network traffic conditions.

Let us consider a flexible OCDMA network with support to multiservice and multirate transmissions with two-user class. The 1-D MWML-OOC [42] is assigned to the users assuming code length and weight and packet length parameters, respective to each class, as given by: $L_{1}=600, W_{1}=4, H_{1}=1024$ bits; and $L_{2}=1200$, $W_{2}=5, H_{2}=512$ bits. The number of users in each class is $U_{1}=36$ and $U_{2}=36$, respectively. The 1-D MWML-OOC employs a single wavelength, then $F=1$. Class- 1 is chosen so that it has low-QoS and high-transmission rate, and class-2 in contrast has high-QoS and low-transmission rate. The packet throughput of the desired class is plotted for both classes as a function of the offered load of the desired class in Figure 4.4 for Poisson (diamonds), binomial (squares) and Markov (circles) approaches. All curves are directly obtained from (3.43), (3.47), and (3.55), respectively. It is worth pointing out that the Markov approach is considered here as a benchmark since it accurately represents the true network performance. The results based on the binomial approach (blue symbols) are in excellent agreement with those based on the Markov chain approach (red symbols), which confirms the reliability of the proposed binomial approach in predicting the performance of flexible OCDMA networks.

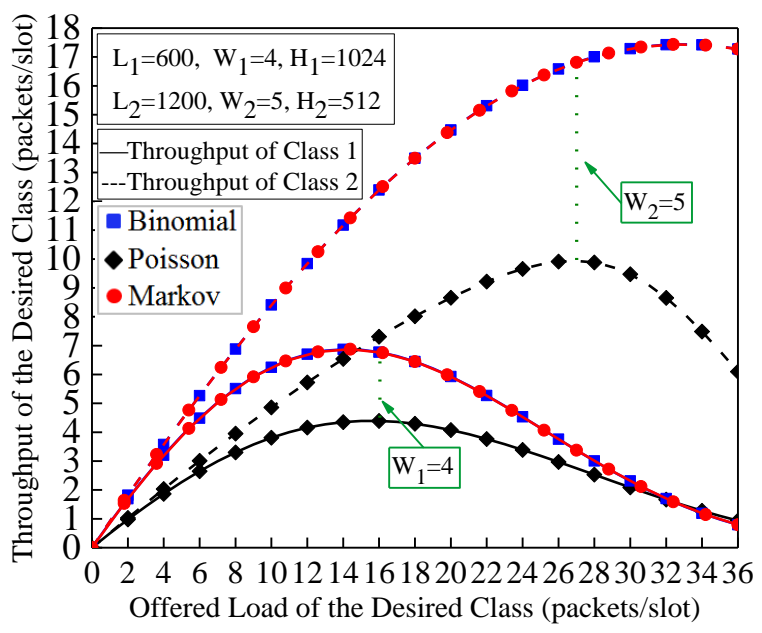

Figure 4.4: Packet throughput of the desired class versus the offered load of the desired class for a two-class 1-D MWML-OOC OCDMA packet network employing binomial (squares), Poisson (triangles), and Markov (circles) approaches. 
Nonetheless, the results based on the Poisson approach only show convergence for class-1 under high offered load conditions, with essentially no agreement with either the binomial or the Markov Chain-based results. Furthermore, it is highly desirable to have the packet throughput as linear as possible since this implies lower probability of receiving packets with errors. In this way, class-2 not only has better throughput performance but also higher throughput peak than class-1. The users of class-1, by its turn, have a low throughput performance since this class has lower code weight, which makes its users more susceptible to the effects of MAI. However, the lower code weight of class- 1 renders the Poisson-based results more accurate than those for class-2 (see dotted vertical lines in Figure 4.4).

It can still be observed from Figure 4.4 that the Poisson approach produces unacceptable results for most values of offered load and underestimates the throughput peak for class- 1 and class-2 users by around $59 \%$ and $73 \%$, respectively. In addition, it underestimates the overall performance of the network in unacceptable levels and incorrectly predicts the packet throughput of class- 2 for any given offered load. Therefore, it can be concluded that for this network scenario, the Poisson approach can only be considered appropriate for throughput performance evaluation of class- 1 only if more than 30 simultaneous users (offered load) are present in the network. Yet, it does not produce accurate results for any value of offered load from class-2.

Next, we proceed with the packet throughput evaluation of flexible networks, this time for a scenario with distinct number of users in each class. Also, different parameters are used where a user class has twice the transmission rate of the other class. Hence, let us consider a flexible OCDMA network with two user-class using the 1-D MWML-OOC, assuming: $L_{1}=400, W_{1}=5, U_{1}=21, H_{1}=3072$ bits for class- 1 , and $L_{2}=1200, W_{2}=6, U_{2}=26$, and $H_{2}=1024$ bits for class-2. Class- 1 is set with lowQoS and high- transmission rate, while class-2 is set with high-QoS and lowtransmission rate.

The packet throughput of class-1 versus its offered load is plotted in Figure 4.5(a) for the three different approaches, namely, binomial (squares), Poisson (triangles) and Markov Chain (circles). As in the previous network scenario, the results based on the binomial and Markov chain approaches are in excellent agreement. In fact, the binomial approach has the same accuracy provided by the Markov chain, which is considered the benchmark against which the performance can be compared. In addition, the investigations carried out so far show that the binomial approach shows good equivalence in terms of results with the Markov Chain and can be considered a reliable alternative to evaluate the packet throughput performance of flexible OCDMA networks. 
The Poisson approach, by its turn, underestimates the throughput performance for most values of offered load, peaking at approximately $G_{1}=10$ where it produces a throughput around 64\% lower than that predicted with the binomial/Markov approach, as can be seen in Figure 4.5 (a) (dotted vertical line). This means that under the Poisson approach the network would receive more packets with errors than it should. Indeed, there is essentially no agreement between the Poisson and Markov Chain approach.

Next, the packet throughput of class- 2 versus the offered load of class- 2 is plotted in Figure 4.5(b), again considering the three proposed approaches. Once again, unlike the Poisson approach, both binomial and Markov-based results are in good agreement. The Poisson approach significantly underestimates the packet throughput performance for classs-2.

For example, the throughput is underestimated by around $75 \%$ at $G_{2}=8$ (dotted vertical line in Figure 4.5(b)), and this discrepancy becomes even more significant for higher offered loads. At $G_{2}=25$, the binomial approach predicts a throughput around 15 packets/slot, while the Poisson-based approach predicts around 7 packets/slot only. This gives an underestimation around $120 \%$ in the number of successfully received packets (dotted vertical line in Figure 4.5(b)). Therefore, the Poisson approach is not appropriate to be used for throughput performance evaluation.

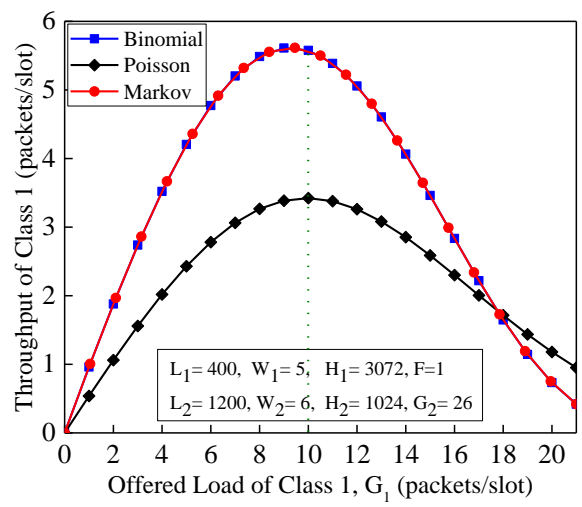

(a)

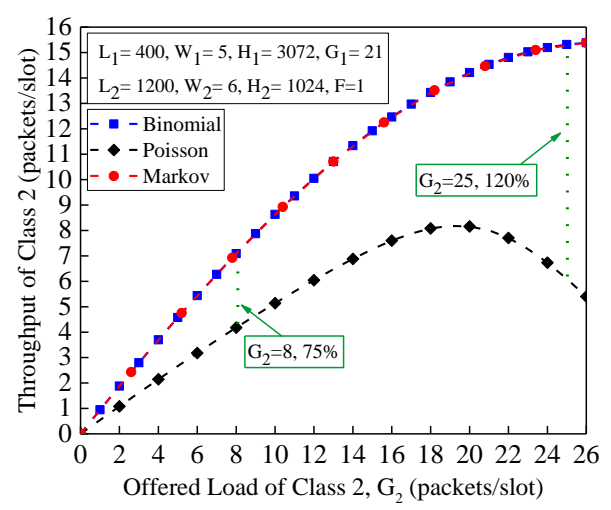

(b)

Figure 4.5: Packet throughput performance of a two-class 1-D MWML-OOC OCDMA network employing binomial (squares), Poisson (triangles), and Markov (circles) approaches. a) Throughput of class- 1 versus the offered load of class-1 (solids). b) Throughput of class-2 versus the offered load of class-2 (dashed). At $G_{2}=8$ and $G_{2}=25$ the Poisson approach underestimates the throughput by around $75 \%$ and $150 \%$, respectively. 


\subsection{2-D MWML-OOC OCDMA}

In this Subsection, the mathematical formalisms developed in Chapter 3 are now applied to address the packet throughput performance of flexible OCDMA networks. Once more, the three proposed approaches (binomial, Poisson and Markov Chain) are assessed and compared under different network's parameters. This time a 2-D OCDMA network with support to a high number of users is considered so that the convergence among the approaches can be further evaluated. Then, let us consider a multiservice, multirate OCDMA network where users are assigned with 2-D MWMLOOC. This code requires a simple construction algorithm and can support a significantly large number of users [54]. The 2-D MWML-OOC OCDMA packet network with two-classes has its parameters defined as follows: $L_{1}=100, W_{1}=4, U_{1}=$ $70, H_{1}=2048$ bits for class- 1 , and $L_{2}=200, W_{2}=5, U_{2}=50$, and $H_{2}=1024$ bits for class2. All users' codes share the same number of available wavelengths used in the design of 2-D MWML-OOC, $F=11$. Class-1 has low-QoS and high-transmission rate while class-2 has high-QoS and low-transmission rate. Even though the transmission rate is normalized and proportional to the ratio of the user classes' code length, one can plausibly reach data transmissions at $5 \mathrm{~Gb} / \mathrm{s}$ for class- 1 and at $2.5 \mathrm{~Gb} / \mathrm{s}$ for class- 2 . These transmissions rate can be achieved by current available encoding schemes and OOK technology. The packet throughput of class-1 versus the offered load of class-1 for the three proposed approaches is plotted in Figure 4.6(a). Once more, notice that the results obtained via binomial (squares) and Markov (circles) approaches are in good agreement and a show excellent overlap. Nevertheless, it is worth mentioning that the binomial approach is numerically more straightforward as well as computationally faster than the Markov chain approach. Both approaches render equivalent results, but the binomial approach requires less time and computational efforts to be run, whereas the Markov is constrained by time consideration and computational expensive.

As an example, the packet throughput expression based on binomial approach only depends on the packet correct probability and on the (binomial) probability distribution itself. On the other hand, the Markov chain-based throughput expression relies on a state transition matrix for each user and, consequently, on the conditional statements, summations and binomial coefficients of its mathematical formalism. Consequently, this formalism requires considerably more computational effort to evaluate the packet throughput. Moreover, the larger the number of users in the network, the larger the square transition matrix and, consequently, the larger the computational demand required to evaluate the throughput. For example, in this 2D network scenario, a $70 \times 70$ and a $50 \times 50$ square transition matrix along with their eigenvalues and eigenvectors are required to obtain the results based on the Markov chain approach shown in Figure 4.6(a) and Figure 4.6(b), respectively. 


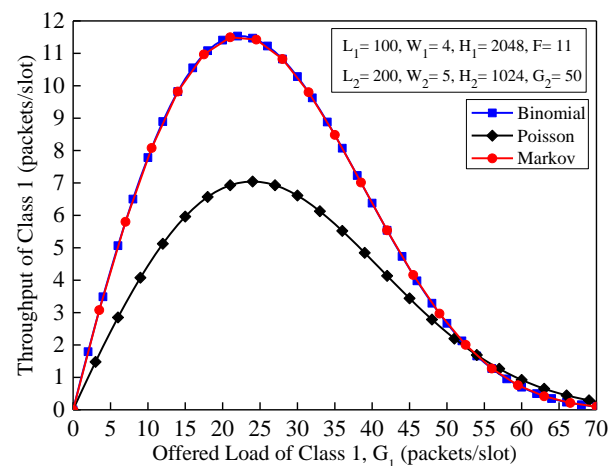

(a)

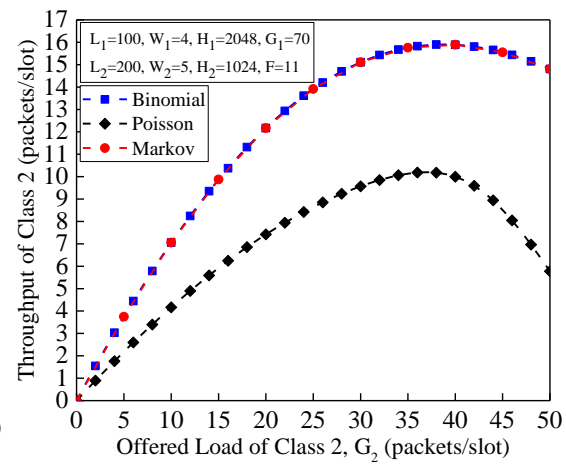

(b)

Figure 4.6: Packet throughput performance of a two-class 2-D MWML-OOC OCDMA packet network employing binomial (squares), Poisson (triangles), and Markov (circles) approaches. a) Throughput of class-1 versus the offered load of class-1 (solids). b) Throughput of class-2 versus the offered load of class-2 (dashed).

Figure 4.6(a) shows that the Poisson approach considerably underestimates the packet throughput performance. For example, the Poisson-based results predicts a throughput around $63 \%$ less than that predicted with binomial/Markov chain approach, at $G_{1}=20$ packets/slot. A good agreement among the three approaches only occurs when $G_{1} \geq 50$, which suggests that the Poisson approach can only be considered sufficiently accurate for this network scenario whether above 50 simultaneous users in class-1 are in the network (see dotted vertical line in Figure 4.6(a)).

The packet throughput of class-2 versus the offered load of class-2 is plotted in Figure 4.6(b). Note once more that both binomial and Markov chain approaches are in excellent agreement whereas the Poisson approach poorly predicts the packet network performance. In such network configuration, the Poisson approach diverges even under conditions of high offered load where the packet throughput underestimation can be as high as $141 \%$, for $G_{2}=50$. Noticeably, the Poisson approach fails to predict correctly the throughput performance of class-2 for any number of offered load. Figure 4.6(b) also shows that there is essentially no agreement between the Poisson and binomial/Markov chain approaches for this network configuration. Therefore, based on the scenarios of network investigated here, it can be concluded that the Poisson based-approach is not appropriate for throughput performance evaluation of flexible OCDMA networks. However, the binomial approach has proven to be as accurate as the Markov chain, with a good compromise between computational cost and accuracy.

Finally, it is worth pointing out that the throughput performance depends on the BER and, consequently, on the code's parameters, on the code family employed, and on 
the number of users in the network. In this sense, the larger the code weight of a user class, the lower the probability of interference caused on the desired user, and the better the BER and throughput performances. The number of simultaneous users also influences the BER and throughput performance since OCDMA networks are considered as statistical multiplexing systems [6].

Furthermore, the network performance can be improved by considering an optimum level of threshold detection based on the MAI power presented in the channel. For example, when the average MAI power is potentially high (with hundreds of simultaneous users accommodated in the network), it is useful to consider threshold values beyond the code weight so that the probability of error related with the data bit " 0 " decreases. It is possible to dynamically estimate the optimum threshold level of flexible OCDMA networks with the help of equation (3.12) and assuming a scenario with possibility of errors for both data bit " 0 " and " 1 ". However, all the scenarios investigated here adopt well-known network scenarios for convenience sake, where only error detection regarding the data bit " 0 " is assumed. In contrast, modelling the MAI in a way that interference among users can be avoided is a mathematically complex problem and is a task that concerns code designers, hence not addressed here.

It is still worth mentioning that although the performance of a three-class network has not been addressed here, one can expect the same performance tendency in terms of convergence among the approaches observed for the two-class networks. In this fashion, the class defined with the highest code weight will eventually have underestimated throughput performance under the Poisson approach. However, Poisson-based results for the class defined with the lowest code weight might converge to the binomial/Markov approach as long as the network has a large number of simultaneous users.

\subsection{2-D FFH-OCDMA}

In this subsection, we evaluate the BER performance of 2-D flexible OCDMA networks where users are assigned with codes based on the FFH scheme employing FBGs as encoders and decoders. The performance comparison is addressed considering separately binomial and gaussian distributions for the MAI. Finally, the results are obtained with the formalism developed in Chapter 3, Section 4.1. Then, let us consider a two-classes OCDMA network with support to multirate transmissions where users are assigned with 2-D FFH-based codes with the following code length and weight, respectively: $L_{1}=12$ and $W_{1}=12$ for the low rate users (class- 1 , circles) and $L_{2}=6$ and $W_{2}=6$ for the high rate users (class-2, diamonds). The number of users 
is $U_{1}=17$ and $U_{2}=6$. The codes of all users share the same number of available wavelengths, with $F=29$. The 29 wavelengths can provide at most 29 code sequences [112]. It should be mentioned that the transmission rate magnitude of the users is transparent to the formalism and is accounted for by means of the code length ratio between the classes of the network. However, one can in a plausive manner achieve data rate transmissions up to $5 \mathrm{~Gb} / \mathrm{s}$ and $2.5 \mathrm{~Gb} / \mathrm{s}$ for class- 1 and class-2, respectively. These transmissions rate can be achieved by the current available encoding schemes as well as OOK technology.

Furthermore, even though the formalism employed in the following numerical simulations assumes MAI as the only noise source, it still allows the possibility of including other noise sources [80] as well as mechanisms to mitigate their influence. With that in mind, the BER versus threshold level for both classes are plotted in Figure 4.7.

Note that the BER is minimized when the threshold level equals the code weight of the desired user, i.e., at $\mu_{o p t_{1}}=12$ (class-1) and $\mu_{\text {opt }}=6$ (class-2), respectively [95]. Despite this, the mathematical formalism developed in Chapter 3, Section 3.1, provides a more general choice of threshold values by treating the threshold as a free parameter to be chosen optimally. This is possible since the threshold value in the BER expression (3.12) can be set to any value, not necessarily the optimal one. As a matter of fact, the optimal threshold cannot always be considered as equal to the weight, even when the MAI is assumed as the only degrading factor of the network [43].

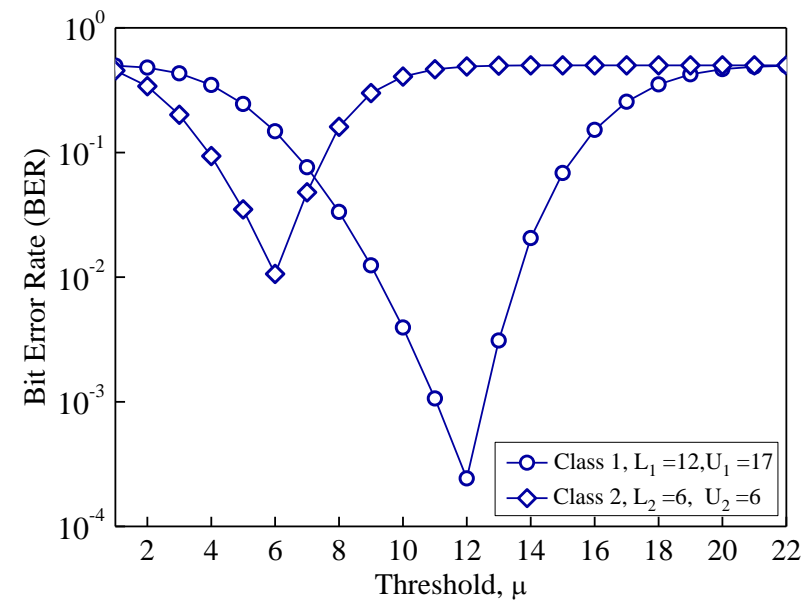

Figure 4.7: Optimum detection threshold with $L_{1}=12, L_{2}=6, U_{1}=17, U_{2}=6$, and $F=29$. The BER of high rate users and low rate users are minimized by choosing threshold values of 6 and 12, respectively. 
Although in most available works the decision threshold value used in the users' detector at the receiver side might be set to the code weight, which ensures that no decision error will be made for bit " 1 " transmissions (whether the channel is considered ideal and additive), in [43] it is reported that this adjustment works well when the MAI is small compared to the code weight. When this is not the case, the probability of error associated with the data bit " 0 " increases when the average MAI power in the optical channel is high. However, this can be alleviated by increasing the threshold value beyond the code weight. In this way, it is of common interest to consider a general choice of threshold by treating the threshold value as a free parameter to be chosen optimally.

For the sake of clarity, the performance investigation carried out here, as already mentioned, is assumed the worst possible scenario for the network evaluation, i.e., chip synchronization and one chip coincidence for any pair of codes. Nonetheless, since the exact performance depends on which codes are interfering on the desired user code, the exact knowledge of the actual interference patterns given by the PDFs is required. An exact calculation of all interference patterns, which would be constrained by computational and complex algebra considerations is also required.

Fortunately, one can bound the effects (means and variances) of these interference patterns by their worst case, namely, chip synchronous and one chip coincidence (each interfering user contributes with one chip to the overlapping), when there are only two users transmitting data simultaneously in the network. When more than two users are simultaneously transmitting, the interference (measured as the mean and variance of the signal) of each interfering user with respect to the desired user becomes the same. Since each interfering signal is identical and an independent random variable, the mean and variance of the total interference signal can be expressed as the product of each mean and variance of the interfering signal.

Lastly, it is well-known from statistics theory that to obtain an acceptable gaussian approximation, the number of trials (number of interfering users) should be large enough, and the probability of success (probability of interference) as close as possible to 0.5 [46], [113], [114]. Otherwise, the gaussian approximation does not converge towards the binomial distribution and consequently does not fulfil its intended purpose. Nonetheless, the MAI should always be considered binomially distributed as only the BER based on the binomial distribution can express true values and accuracy of results. With that in mind, let us now proceed with the comparison analysis between gaussian and binomial distributions for the BER performance of flexible OCDMA networks employing 2-D FFH-based codes. 
The BER versus the number of class-1 users for a two-class network is plotted in Figure 4.8. Notice that the BER performance of class-2 (high rate class, diamonds, solid line) is severely affected by MAI when compared to that for class-1 (low rate class, circles, solid line). This is due to the smaller code weight of class-2 than that used in class-1 [47], [95]. One can further notice from Figure 4.8 that as the number of simultaneous users decreases, the gaussian-based BER curve drops more rapidly than that obtained with binomial distribution. Yet, the approximated results for class-2 (diamonds, dashed line) is more accurate than those for class-1 (circles, dashed line), which is due to the smaller threshold value $\left(\mu_{o p t_{2}}=6\right)$ of the former [46]. One can also notice that the gaussian based-BER underestimates the network performance in unacceptable levels and incorrectly predicts the number of simultaneous users for any given BER. Differently from the binomial approach, the gaussian-based BER is not appropriate for BER performance evaluation of flexible 2-D FFH-OCDMA networks.

Still regarding the network scenario evaluated in Figure 4.8, it is worth pointing out that the BER curves for class-1 (circles, lines) interestingly starts at a number of simultaneous users above the code weight $W_{1}$ plus one $\left(W_{1}+1\right)$. This means it is necessary at least $W_{1}+1$ simultaneous users in the network for an error eventually to occur [79]. This occurs because MAI is the only degrading factor, the detection threshold is set to the code weight $W_{1}$, the codes have good auto- and crosscorrelation properties and errors occur only for the data bit " 0 " transmission. For example, errors start occurring only at $U_{1}=7$ (plus $U_{2}=6$ ) considering $W_{1}=12$ in Figure 4.8, which means that before this point no errors occur for class-1.

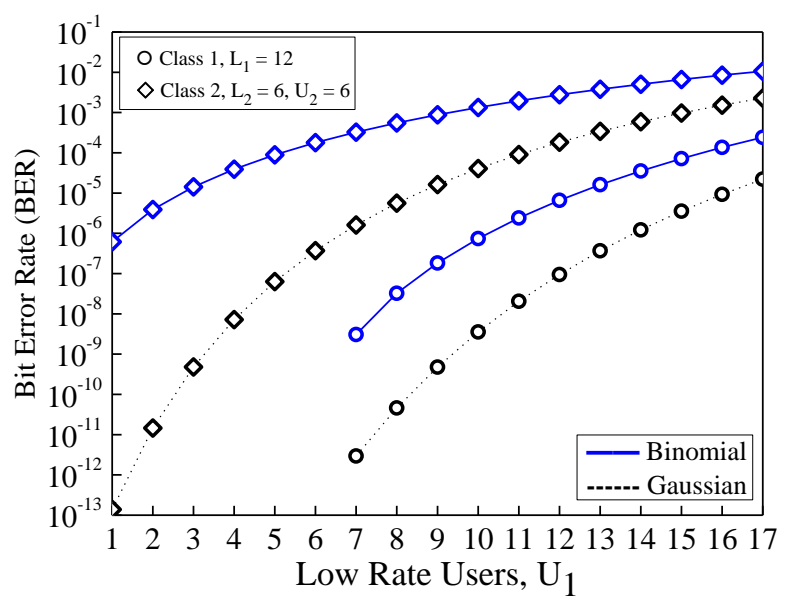

Figure 4.8: BER versus the number of simultaneous class-1 users for a two-class network with $L_{1}=12, U_{1}=17, L_{2}=6, U_{2}=6$ and $F=29$. The number of users in class-1 is varied from 1 to 17 . Binomial approach (solid lines) and gaussian approximation (dashed lines). 
The same is valid for class-2, where errors only occur when the number of simultaneous users is above $W_{2}+1$. Thus, errors occur at $U_{1}=1$ for $W_{2}=6$ in Figure 4.8 since there are already 6 fixed users from class-2 $\left(U_{2}=6\right)$ in the network, which gives a total of 7 simultaneous users at $U_{1}=1$.

Next, the previous two-class network has again its BER performance evaluated, but this time with a smaller number of users in class-2, i.e., $U_{2}=4$. The main goal is to investigate whether the gaussian approximation can worsen its convergence level even further. In this way, the BER performance against the number of simultaneous class-1 users is plotted in Figure 4.9. It can be clearly seen that the gaussian approximation performs poorly under a smaller number of simultaneous users.

Figure 4.9 shows that there is essentially no agreement between both results. In addition, the only evident change is in the position at which errors begins to occur. Accordingly, the smaller number of class-2 users reduces the overall MAI contribution and thus allows a larger number of users in class- 1 before errors begin to appear. This can be a simple way of including new users in a certain class if the demand for transmission rate increases. For example, in Figure 4.8 the number of simultaneous class- 1 users for $B E R=10^{-9}$ is about 9 (dashed line, circles), while in Figure 4.9 it is 11 (dashed line, circles). Again, although these results appear consistent with what one would expect, the inaccuracy in predicting the BER performance or the number of simultaneous users when compared to the binomial based-distribution makes the gaussian approximation completely inadequate for this purpose.

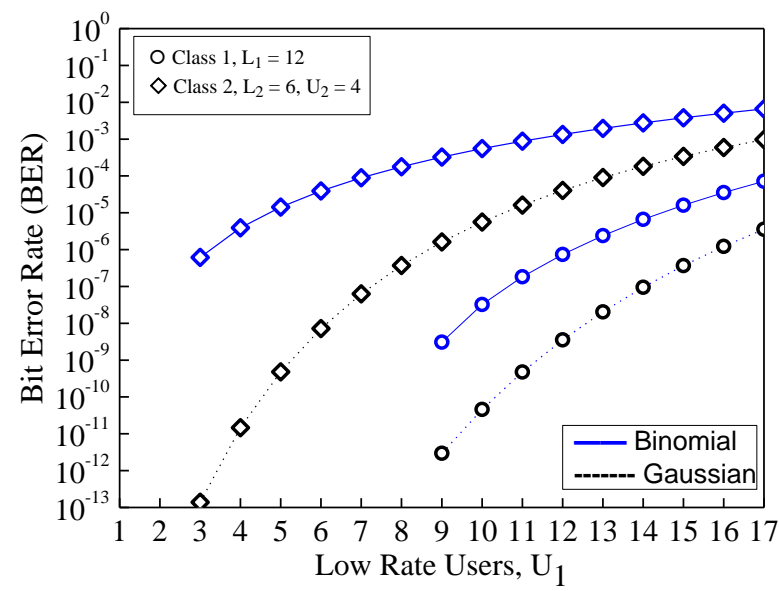

Figure 4.9: BER versus the number of simultaneous class- 1 users for a two-class network with $L_{1}=12, L_{2}=6$, and $F=29$. The number of class- 1 users is varied from 1 to 17 . Class- 2 has only 4 users, $U_{2}=4$. Binomial approach (solid lines) and gaussian approximation (dashed lines). 


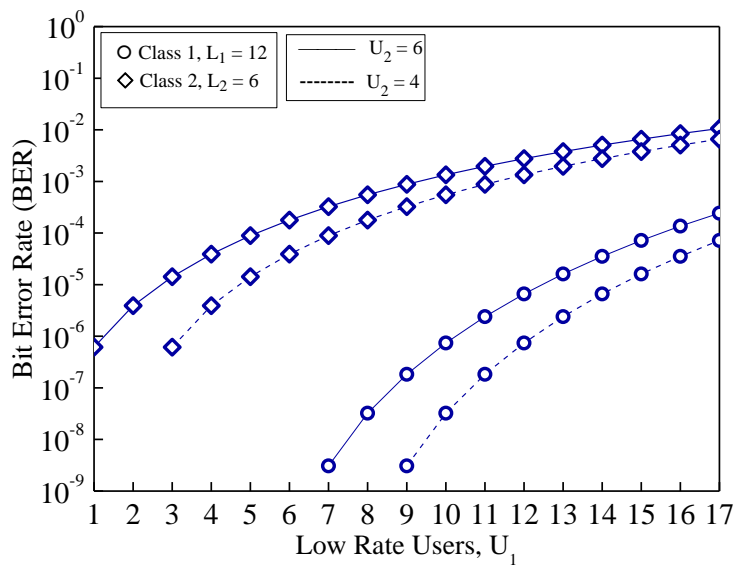

Figure 4.10: BER versus the number of simultaneous class-1 users for a two-class network with $L_{1}=12, L_{2}=6$, and $F=29$. The number of class- 1 users is varied from 1 to 17 . The number of class2 users is either $U_{2}=4$ or $U_{2}=6$. Only results for the binomial approach are shown.

Finally, for the sake of clarity, the BER versus the number of class- 1 users (low rate users) is replotted in Figure 4.10 only for the binomial based-BER equation. Notice that the BER performance of both class- 1 and class- 2 changes noticeably, even though the number of simultaneous users is only slightly reduced. In addition, note that the presence of just two more users in class-2 $\left(U_{2}=6\right.$, solid line) is enough to change the BER by nearly one order of magnitude for most class- 1 users (compare with $U_{2}=4$, dashed line). Therefore, it can be concluded that increasing the number of high rate users can have a considerable impact on the performance of flexible, multirate 2-D FFH-OCDMA networks.

\subsection{2-D MWML-OOC OCDMA, Multicode Technique}

The analytical formalism developed in Subsection 3.3.4 is now applied to the performance evaluation of flexible OCDMA packet networks supporting multirate transmissions via multicode techniques. This technique, as detailed described in Subsection 2.5, assigns several different codes of fixed length to each user of the network so that higher transmission rates can be achieved. Moreover, the BER and packet throughput performance are compared against the conventional multilength code technique, where a single code of non-fixed length is assigned to each user of the network to achieve different transmission rates. Then, let us define an OCDMA packet network with two-user class, where class-1 has high -QoS and -rate, and class2 has low -QoS and -rate. 
The network employs the 2-D MWML-OOC [54] for implementation of both techniques (multicode and multilength code), but since the multicode technique employs code of the same (fixed) length, the code set can also be referred to as 2-D MWSL-OOC (multiweight single-length) [42]. For the sake of clarity, the techniques are implemented separately. Hence, the following parameters are adopted for the multilength code technique: $L_{1}=100, W_{1}=11, U_{1}=40, H_{1}=1024$ bits, $B_{1}=1, L_{2}=200, W_{2}$ $=8, U_{2}=25, H_{2}=512$ bits, $B_{2}=1$, where the subscripts 1 , and 2 refer to a specific class. By its turn, to provide the same QoS and rate, the following parameters are adopted for the multicode technique: $L_{1}=200, W_{1}=11, U_{1}=40, \mathcal{H}_{1}=512$ bits, $B_{1}=2, L_{2}=200, W_{2}$ $=8, U_{2}=25, \mathcal{H}_{2}=512$ bits, $B_{2}=1$, where the subscripts 1 , and 2 refer to a specific class. The threshold value is set to the respective user's code weight of each class. All users' codes, for both multirate techniques, share the same number of available wavelengths, $F=19$. In addition, in both techniques, class- 1 users transmit the same number of bits, since each multicode user transmits two packets simultaneously.

The BER performance for both classes versus the number of simultaneous class- 1 user's codes are plotted in Figure 4.11, for both multirate techniques (multilength code 2-D MWML-OOC, dotted lines, and multicode 2-D MWSL-OOC, solid lines). As shown in this figure, the multicode technique has better BER performance than does the multilength code. This is due to the multiple codes assigned to class- 1 users as well as the larger code length employed in the multicode technique, which allows a reduction in the probability of interference on the desired user class. One can still observe from this figure that the BER performance of class- 1 is better than that of class-2. This is expected since class- 1 has higher code weight than class-2.

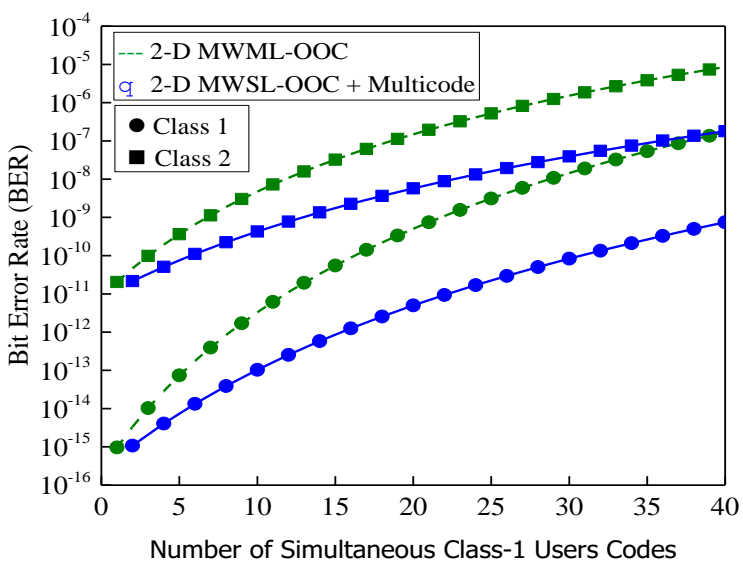

Figure 4.11: BER performance of the desired user-class versus the simultaneous number of class1 users' codes for a two-class network and for both multicode (solid lines) and multilength code techniques (dotted lines). 


\section{NUMERICAL RESULTS AND DISCUSSIONS}

Figure 4.12 shows that the multicode technique outperforms the multilength code. It is highly desirable to have the throughput performance as linear as possible since this implies lower probability of receiving packets with errors. Accordingly, class- 1 users under the multicode technique (solid line) have better throughput performance than class-1 users under the multilength technique (dashed line). Under higher load conditions, the throughput based on the multilength technique peaks and starts decreasing more abruptly than the multicode-based one, which is essentially linear. This suggests that, under the multicode technique, more packets are being received with success in the network than it does under the multilength technique.

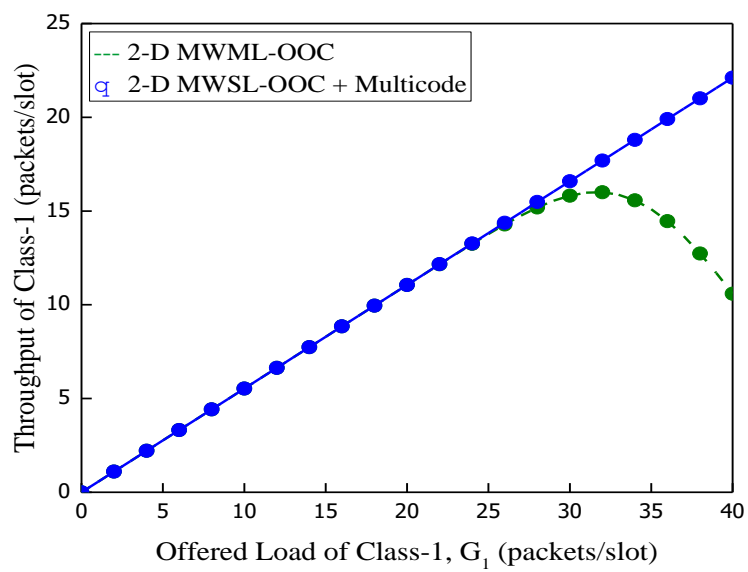

Figure 4.12: Packet throughput of class-1 versus the offered load of class-1 for a two-class network for both multicode and multilength code techniques.

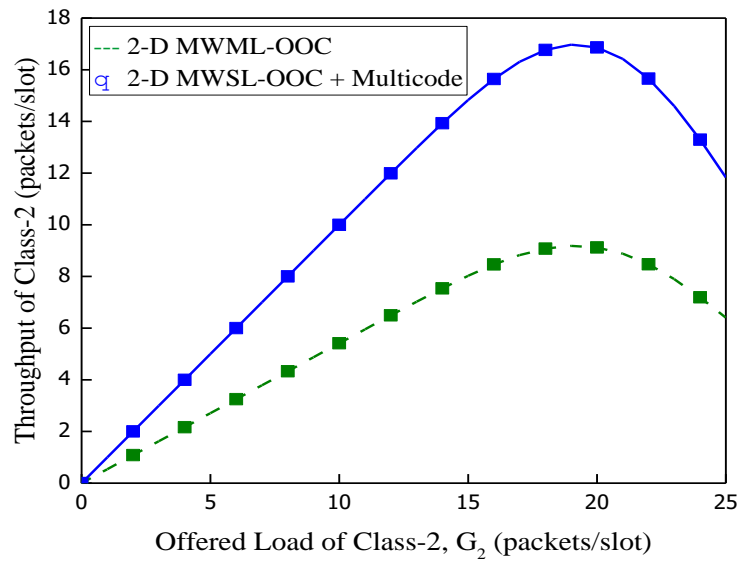

Figure 4.13: Packet throughput of class-2 versus the offered load of class-2 for a two-class network for both multicode and multilength code techniques. 
Subsequently, the packet throughput of class-2 versus the offered load of class-2 is plotted in Figure 4.13, again for both multirate techniques. Once more, the multicode technique (solid line) has better performance than the multilength code technique (dashed line). This means that under the multilength code the network receives more packets with errors than it does with the multicode technique. The 2-D MWML-OOC multilength code has a poor throughput performance since users employing this technique are more susceptible to the effects of MAI. In addition, although both throughput curves peak at around the same offered load value $\left(G_{2} \approx 19\right)$, the multicode-based throughput is more linear than the multilength-based one. Consequently, users employing the multicode technique transmit more packets with success. For example, at $G_{2} \approx 19$ in Figure 4.13 , the multicode technique predicts a throughput around 17 packets/slot whereas the multilength code predicts around 9 packets/slot only, which represents a reduction of $88 \%$ in the number of successfully received packets.

\subsection{1-D MWML-OOC IP OCDMA}

The analytical formalism developed in Subsection 3.2 is now applied to the performance evaluation of IP OCDMA packet networks supporting multiservice and multirate transmissions. The architecture of the IP traffic OCDMA network addressed here was thoroughly described in Subsection 4.2. The packet error rate or PER performance of two different network scenarios is addressed, where the latter has its channel utilization varied from 0.2 to 1 . Then, let us consider a flexible IP traffic OCDMA network with support to multiservice and multirate transmissions with three-user classes. The users of the network are assigned with 1-D MWML-OOC assuming code length and weight, and number of users, respectively, as: $L_{1}=500, W_{1}$ $=7, U_{1}=4$ (diamonds), $L_{2}=1000, W_{2}=5, U_{2}=20$ (squares), and $L_{3}=1500, W_{3}=3, U_{3}=$ 20 (circles), where the subscripts 1,2 and 3 refer to a specific class. In addition, a packet length $H=12000$ bits is assumed, i.e., Ethernet local area network maximum packet length, which reflects the worst possible case for packet length condition [99], [100]. The variation of the channel utilization can change the number of simultaneous users in the network. Consequently, the channel utilization will have a significant effect on the performance of the network, since it is a function of the number of active users [99]. To analyse this effect, it is assumed that all users have the same channel utilization, i.e., fixed $B j$. For example, when channel utilization is $40 \%(B j=0.4)$ in Figure 4.14, all three classes will have the same value of $B j$ and their channel utilization varied at the same instance. This is considered for all simulations.

The PER performance for all classes versus number of simultaneous class- 3 users is plotted in Figure 4.14 considering two different values of channel utilization. 


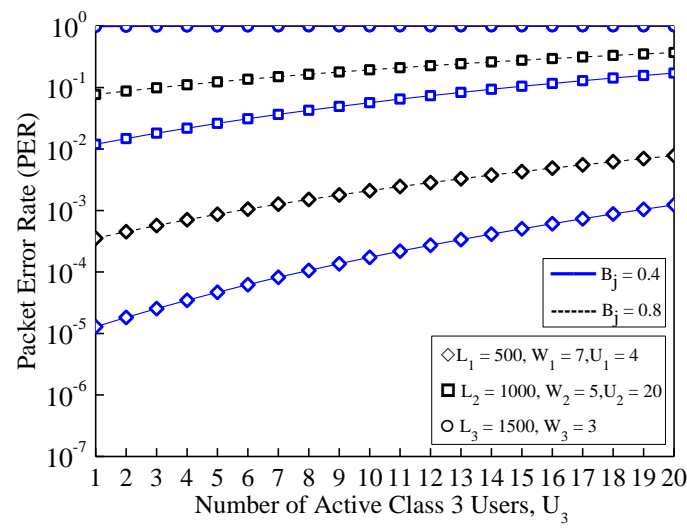

Figure 4.14: PER performance for all classes versus number of class-3 simultaneous users for a three-class 1-D OCDMA IP packet network. The channel utilization is considered either 0.4 (solid lines) or 0.8 (dashed lines).

It can be noticed that class-3 (circles) has inferior performance compared to the other classes, which occurs due to its lower code weight. Moreover, increasing the number of active users in class-3 has a small effect on the overall performance of the network because of class-3's low probability of interference [42]. One can further observe that the network performance has $P E R=1$ for class-3, which means that errors occur for every single transmitted packet. Also, one can still notice that when the channel utilization changes from $80 \%(B j=0.8)$ to $40 \%(B j=0.4)$, the improvement of the PER performance for class- 1 is relatively large when compared with class- 2 .

Next, let us consider a flexible IP traffic OCDMA network with two-user classes employing 1-D MWML-OOC with the following parameters: $L_{1}=500, W_{1}=7, U_{1}=10$ for class-1 (high-QoS and -rate users), and $L_{2}=1500, W_{2}=5, U_{2}=22$ for class-2 (lowQoS and -rate users), respectively. The goal here is to investigate the impact of the channel utilization variation on the network performance. The PER performance versus number of users in class- 2 is shown in Figure $4.15 \mathrm{a}$ and Figure $4.15 \mathrm{~b}$ considering a variation of the channel utilization from 0.2 to 1 for both classes. One can note from Figure 4.15a that class-1 (solid lines), unlike class-2 (dashed line), performs better for all cases of channel utilization when compared with the worst channel utilization condition of class-2 ( $B_{2}=1$, triangles, solid line).

One can observe from Figure $4.15 \mathrm{~b}$ that for the worst case of channel utilization, i.e., $100 \%$ channel utilization, the performance of class-1 (triangles, solid line) is better than that of class-2 (triangles, dashed line). This is due to the larger code weight used in class- 1 than that used in class-2. One can also observe that class- 1 with $B_{1}=1$ (triangles, solid line) has better performance than class-2, even when class-2 has only $40 \%$ of channel utilization (diamond, dashed line). 


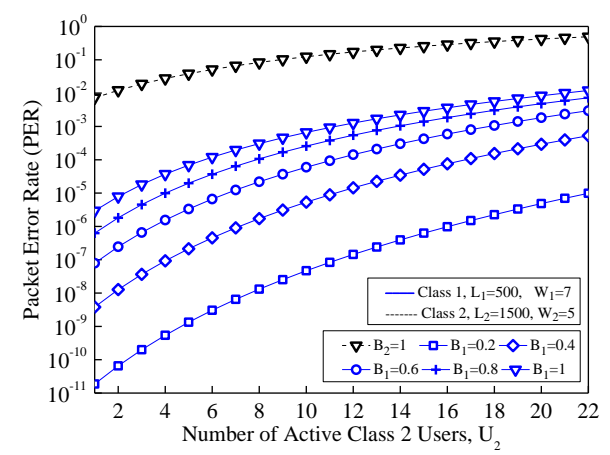

(a)

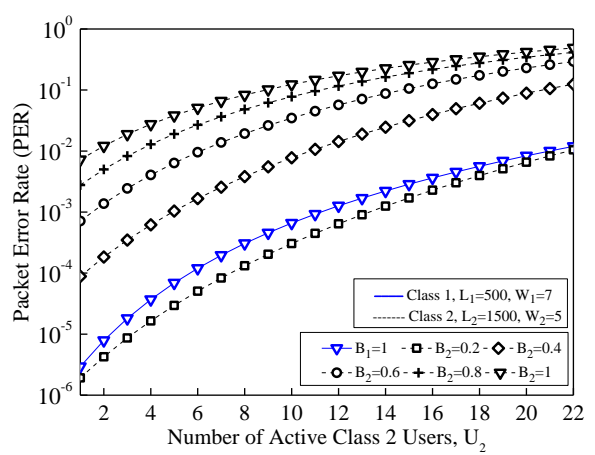

(b)

Figure 4.15: PER performance versus the number of active class-2 users for a two-classes 1-D OCDMA IP packet network. The number of class 1 users is fixed to $U_{1}=10$. The channel utilization is varied from 0.2 to 1 (0.2 spacing) for both classes accordingly. a) Class- 1 results. b) Class-2 results.

It can be further noticed that class-2 (squares, dashed line) only presents a performance better than class-1 (triangles, solid line) when its channel utilization is low $\left(B_{2}=0.2\right)$. Finally, it can also be noticed that the overall PER performance is improved for both classes when decreasing the channel utilization.

\section{8-D FFH IP OCDMA}

In this subsection, the mathematical formalism developed in Subsection 3.2 is applied to address the decoder, BER, total BER, PER and packet throughput performance of flexible IP OCDMA networks, where users are assigned with codes based on the 2-D FFH scheme employing FBGs as encoders and decoders. The performance evaluation is addressed considering only the binomial distribution for the MAI and the Poisson distribution for the composite packet arrivals. Despite the poor approximation given by the Poisson distribution, it is the first time that such a packet throughput evaluation is carried out for a flexible IP OCDMA packet network. Then, let us consider a flexible IP OCDMA packet network with support to multirate transmissions, where users are divided into two-classes and assigned FFH-based codes assuming code length and weight, respectively, as $L_{1}=6, W_{1}=6$, $L_{2}=12, W_{2}=12$, where the subscripts 1 and 2 refer to a specific class. The number of users in each class is defined as $U_{1}=12$ and $U_{2}=17$, respectively. In addition, for the code design it is considered a number of available wavelengths equals $F=29$ [95], and the total number of users in the network is $U=29$. 


\section{NUMERICAL RESULTS AND DISCUSSIONS}

The decoder BER versus number of simultaneous class- 2 users for both classes are plotted in Figure 4.16. Notice that class-2 users (solid line) perform better than class1 (dashed line) users. This occurs because class-2 users are less susceptible to MAI since they have higher code weight than class-1 users. Although neither class- 1 nor class-2 can achieve error-free transmissions (BER $\leq 10^{-12}$ ), both classes can still support users with error-free transmissions if a forward error correction (FEC) algorithm such as the continuously interleaved Bose-Chaudhuri-Hocquenghem (CI-BCH) is employed. The CI-BCH FEC only works properly if the original decoder BER is below the minimum level required $\left(\mathrm{BER} \leq 4.5 \times 10^{-3}\right)[115]$.

Before proceeding with the network performance analysis, a packet length of 12000 bits for all simulations is again assumed, i.e., Ethernet local area network maximum packet length. This reflects the worst possible case for packet length condition [99], [100]. The total BER of class- 1 as function of the number of simultaneous class- 2 users is plotted in Figure 4.17a. The channel utilization is varied for both classes at the same instance from 0.2 up to 1.

The results show that the overall BER performance improves as the channel utilization decreases, and that class- 1 only has a reasonable good performance when the channel utilization is very low, i.e. $B_{1} \leq 0.4$. Subsequently, the total BER of class- 2 as a function of the number of simultaneous class-2 users is plotted in Figure $4.17 \mathrm{~b}$. It can be observed from both figures (a) and (b) that class-2 (solid lines) outperforms class-1 (dotted lines) for any value of channel utilization. It can be further observed that under the worst channel utilization case, class-2 (triangles in Figure 4.17b) outperforms class-1 (crosses in Figure 4.17a) in terms of total BER for a large range of simultaneous users in class-2, even when $B_{1}=0.2$ in class- 1 .

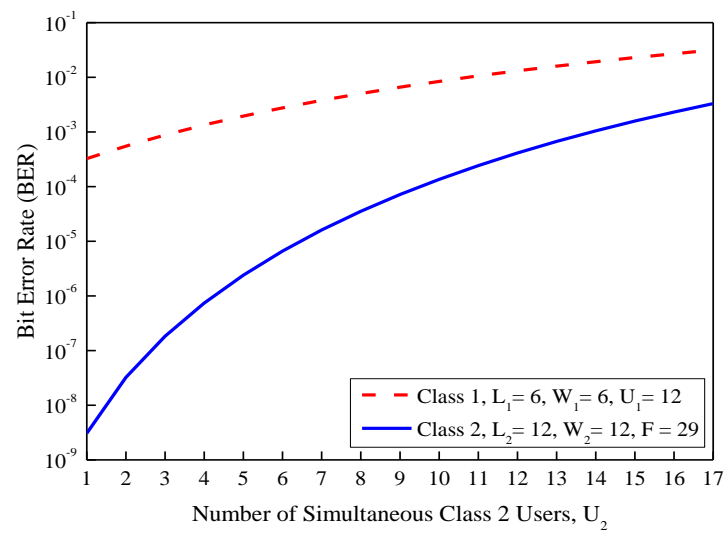

Figure 4.16: Decoder BER performance for Class-1 (dashed line) and Class-2 (solid line) of a flexible IP OCDMA packet network based on 2-D FFH scheme. The number of users in class-2 is varied from 1 to 17 . 
The performance difference between both classes becomes more evident, particularly if a standard BER scenario is desirable (BER $\leq 10^{-9}$, see horizontal dotted line in Figure $4.17 \mathrm{~b}$ ). In such case, class-2 can support all simultaneous users under low channel utilization $\left(B_{2}=0.2\right)$, and can support up to 3 simultaneous users in the standard BER level under the worst channel utilization scenario $\left(B_{2}=1\right)$. On the other hand, class-1 (Figure 4.17a) does not support any simultaneous users in the standard BER level.

The PER of class-1 versus number of simultaneous class-2 users is plotted in Figure 4.18a for different values of channel utilization. It is assumed that the classes have their channel utilization varied simultaneously. This figure shows that the PER's levels are considerably high, especially when $B_{1}>0.2$. Moreover, many values of simultaneous users have PER $=1$, which is even more evident under higher channel utilizations. Although not desired, this may occur under conditions of high channel utilization as also observed in [100]. The poor PER performance of class- 1 is due to the high BER levels (dashed line, Figure 4.16) obtained at the decoders of the multirate network, which results in several packets being received with bit errors.

The PER of class- 2 as a function of the number of simultaneous class- 2 users is shown in Figure 4.18b. The low BER level (solid line, Figure 4.16) obtained at the decoders of class-2 users is responsible for the much lower PER of this class when compared to class-1. Even under the worst channel utilization case, class-2 users (triangles in Figure 4.18b) have better overall PER performance than class-1 users do (cross symbols in Figure 4.18a), when the latter has $B_{1}=0.2$.

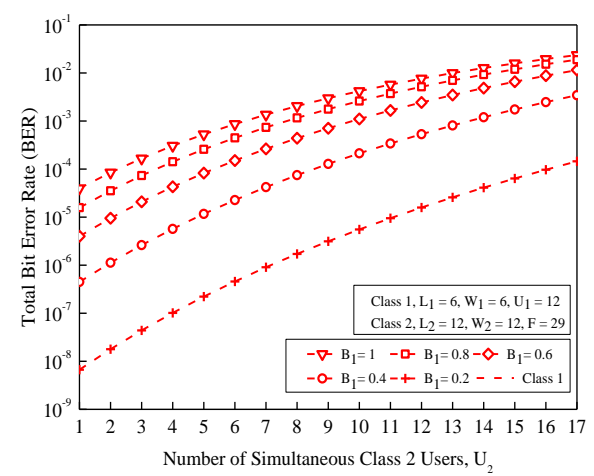

(a)

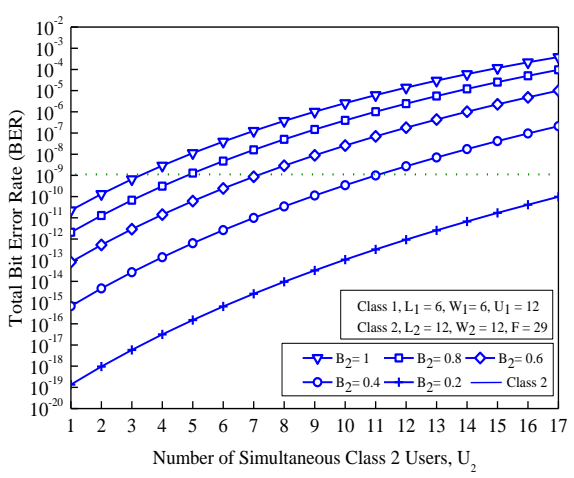

(b)

Figure 4.17: Total BER performance of a flexible IP OCDMA packet network based on 2-D FFH scheme for different values of channel utilization and $H=12000$ bits. a) BER of class- 1 versus number of simultaneous class-2 users. b) BER of class- 2 versus number of simultaneous class- 2 users. The channel utilization is varied from 0.2 to 1 ( 0.2 spacing) for both classes. The dotted horizontal line indicates the standard BER limit region. 


\section{NUMERICAL RESULTS AND DiSCUSSIONS}

The throughput performance of the two-class IP OCDMA packet network is now evaluated for an offered load of $G_{1}=12$, and $G_{2}=17$, respectively. Hence, the packet throughput of class-1 versus offered load of class-1 is plotted in Figure 4.19(a) which shows that the packet throughput performance changes considerably when the channel utilization is varied. One can further notice that as the channel utilization is reduced, the throughput curve takes longer to peak. A throughput curve as linear as possible is highly desirable since it implies lower probability of receiving packets with errors.

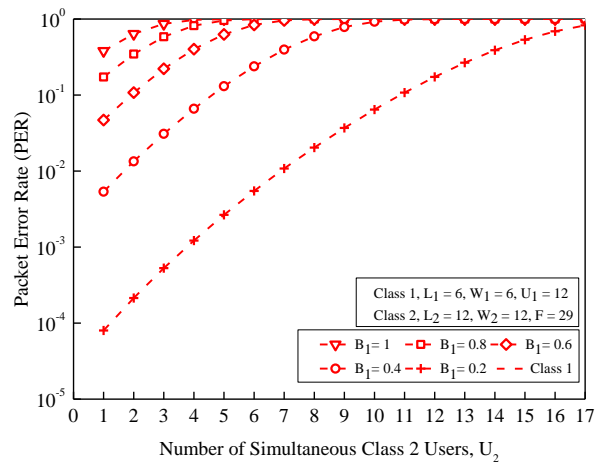

(a)

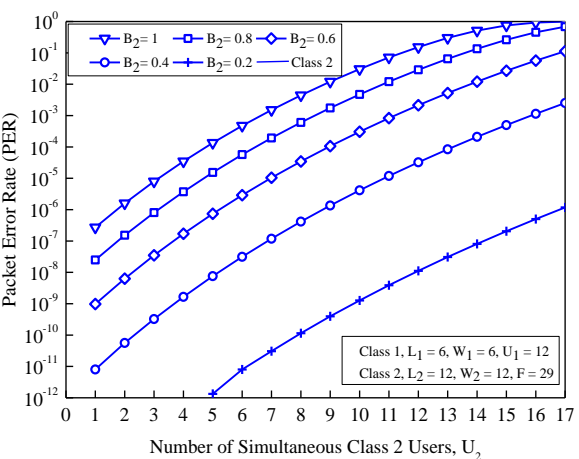

(b)

Figure 4.18: PER performance of a flexible IP OCDMA packet network based on 2-D FFH scheme for different values of channel utilization and $H=12000$ bits. a) PER of class- 1 versus number of simultaneous class-2. b) PER of class-2 versus number of simultaneous class-2 users. The channel utilization is varied from 0.2 to 1 ( 0.2 spacing) for both classes.

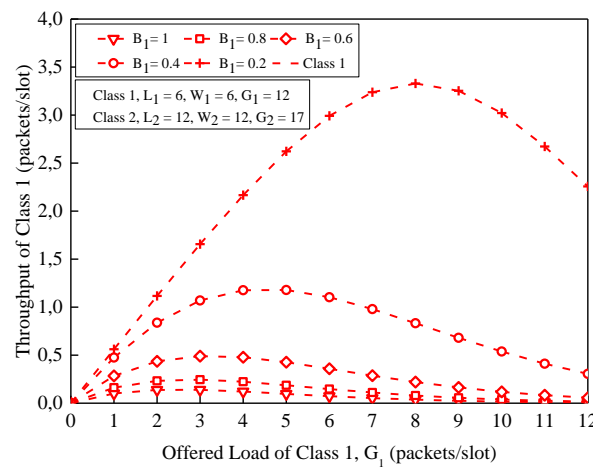

(a)

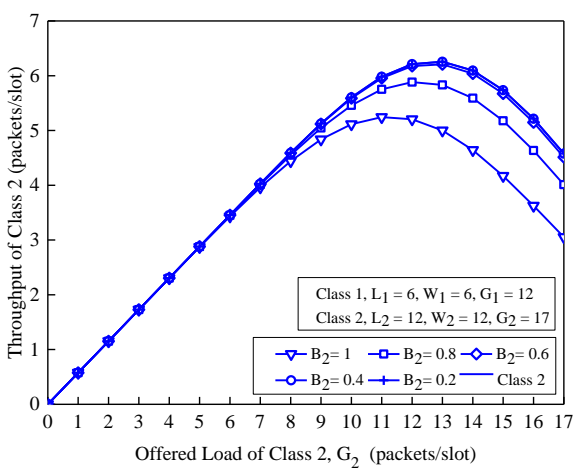

(b)

Figure 4.19: Throughput performance of a flexible IP OCDMA packet network based on 2-D FFH scheme for different values of channel utilization and $H=12000$ bits. a) Throughput of class-1 versus the offered load of class-1. b) Throughput of class- 2 versus the offered load of class-2. The channel utilization is varied from 0.2 to 1 ( 0.2 spacing) for both classes. 
Accordingly, the best throughput performance of class- 1 is achieved when $B_{1}=0.2$ (crosses). The throughput performance of class- 1 is considerably low under the worst channel utilization case, as can be observed in Figure 4.19a. This means that most IP packets are received with bit errors due to MAI. Finally, the throughput of class-2 as a function of the class- 2 offered load is plotted in Figure $4.19 \mathrm{~b}$, also for different values of channel utilization. Observe that class-2 has a better overall throughput performance than class-1 (Figure 4.19a). Moreover, the throughput curves overlap for $B_{2}=0.2$ and $B_{2}=0.4$. Remarkably, the throughput performance of class- 2 changes only for $B_{2}>0.6$ as the channel utilization is modified. After exhaustive tests, it can be concluded that this is due to the considerable low decoder BER level of class-2 (Figure 4.16). This suggests that class-2 (low-rate users) is less susceptible to variations of channel utilization than class-1 (high-rate users) is.

\subsection{2-D MWML-OOC OCDMA, Packet Delay}

The mathematical formalisms developed in Subsection 3.4 are now applied to address the packet throughput and packet delay performance of flexible OCDMA networks. The three proposed approaches, namely, binomial, Poisson and Markov Chain are assessed and compared under different network's parameters. Let us consider a flexible OCDMA packet network, where users are divided into two-classes and assigned 2-D MWML-OOC with the following parameters: $L_{1}=100, W_{1}=8, U_{1}=64$, $H_{1}=1024$ bits, $L_{2}=200, W_{2}=7, U_{2}=32, H_{2}=512$ bits, where the subscripts 1 and 2 refer to a specific class. The threshold value is set to the respective user's code weight of each class and all users share the same number of available wavelengths, $F=11$. The users are divided so that class- 1 has high -QoS and -rate, and class- 2 has low -QoS and -rate. Hence, the packet throughput of class-1 is plotted versus the offered load of class-1 in Figure 4.20a for Poisson (circles), binomial (squares) and Markov (diamonds) approaches. Again, the Markov approach is considered as benchmark.

As can be seen in Figure 4.20a, both the binomial and Markov-based results completely overlap whereas the Poisson ones diverges from them. The Poisson approach underestimates the network performance and incorrectly predicts the throughput for any given value of offered load. The throughput of class- 2 versus the offered load of class-2 is plotted in Figure $4.20 \mathrm{~b}$. Again, notice the overlap of both the binomial and Markov chain approaches. The former can be employed as a reliable approach to accurately evaluate the throughput performance of flexible OCDMA networks. By its turn, the Poisson approach underestimates the throughput performance for the entire range of offered load, predicting a throughput lower than that achieved with the binomial/Markov approaches. 


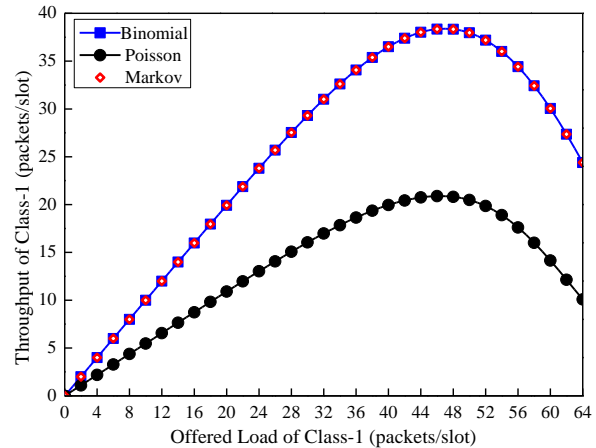

(a)

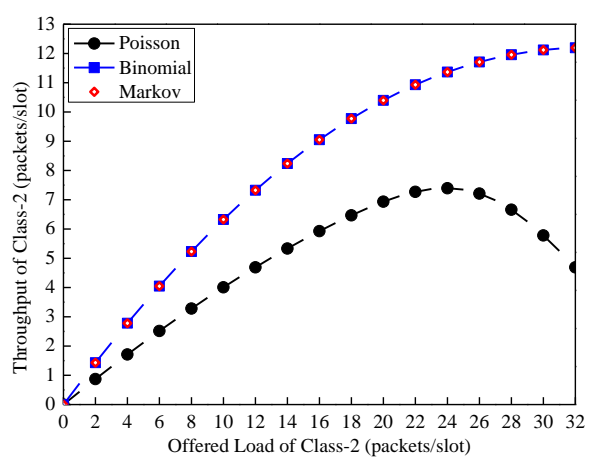

(b)

Figure 4.20: a) Throughput of class-1 versus the offered load of class-1 for a two-class flexible OCDMA network. b) Throughput of class-2 versus the offered load of class-2 for a two-class flexible OCDMA network.

In this fashion, class-1 not only has better throughput performance but also higher throughput peak than class-2 does. Class-2, by its turn, has a poor throughput performance since it has lower code weight, which makes this users' class more susceptible to the MAI effects. The average delay of class- 1 is plotted versus the offered load of class- 1 for the Poisson, binomial and Markov chain approaches. The Poisson approach, as expected, overestimates the delay of the flexible OCDMA network. This happens since the packet throughput is considerably underestimated when compared to the binomial/Markov approach (see Figure 4.20a). Putting it simply, the Poisson approach predicts more delayed packets than should be expected.

Next, the delay of class-2 versus the offered load of class-2 is plotted in Figure 4.21b, again for the three proposed approaches. Both the binomial and Markov curves are in good agreement, while the Poisson approach significantly overestimates the packet delay performance. For example, for high values of offered load the delay under the Poisson approach keeps on increasing whereas it smoothly grows under the binomial approach. Furthermore, both Figure $4.21 \mathrm{a}$ and Figure $4.21 \mathrm{~b}$ show that class- 2 presents higher delay when compared to class-1, which is due to the lower throughput level of class-2.

The average delay of class- 1 is plotted in Figure 4.22a as a function of the throughput of class- 1 for the three approaches. Note that the delay is highly overestimated by the Poisson approach which predicts a higher delay than that achieved with the binomial/Markov approach. In contrast, both the binomial and Markov curves completely overlaps. Notice that the highest delay is the same as that obtained in Figure 4.21a. For example, the value obtained with the binomial is around 2.7 slots in both Figure 4.21a and Figure 4.22a. 


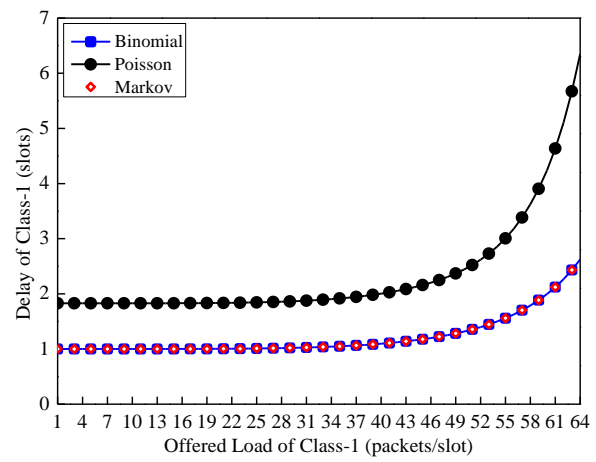

(a)

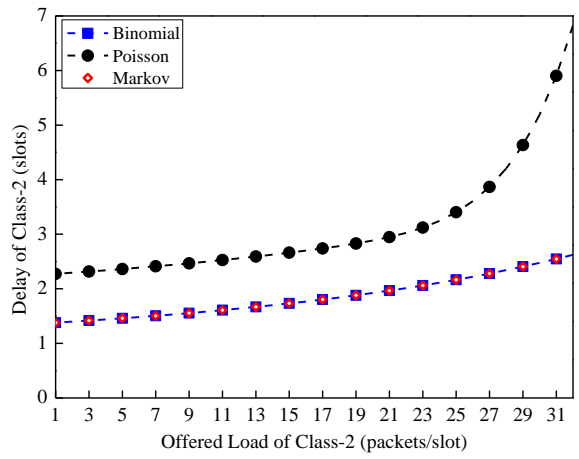

(b)

Figure 4.21: a) Delay of class-1 versus the offered load of class-1 for a two-class network employing binomial, Poisson, and Markov approaches. b) Delay of class-2 versus the offered load of class-2 for a two-class flexible OCDMA network for three different approaches, namely the binomial (squares), Poisson (triangles) and Markov chain (circles) approaches.

Finally, the average delay of class-2 is plotted in Figure $4.22 \mathrm{~b}$ as a function of the throughput of class-2 for the three approaches and, yet again, the Poisson-based approach does not predict correctly the delay of the flexible network. In fact, the calculated delay increases abruptly whereas for the binomial/Markov-based curves (which completely overlaps) it stops at around 3 slots, which is the same as observed in Figure 4.21b.

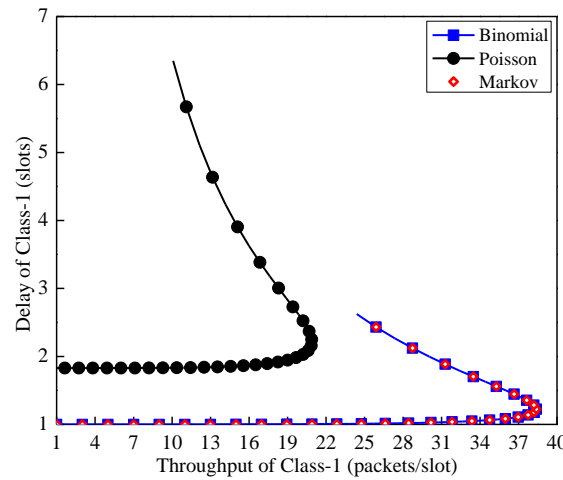

(a)

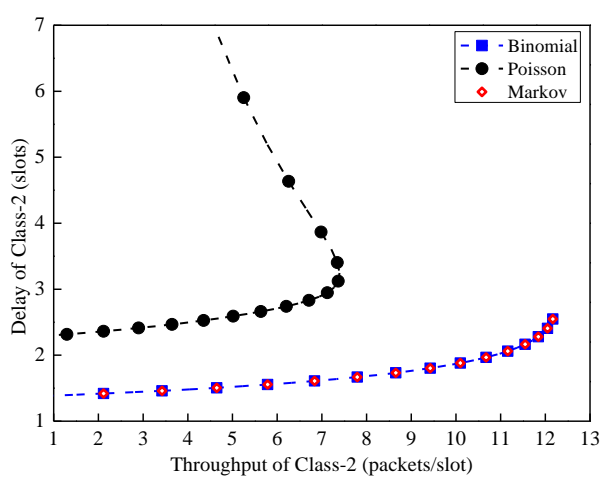

(b)

Figure 4.22: a) Delay of class-1 versus the throughput of class-1 for a two-class flexible OCDMA network for three different approaches, namely the binomial (squares), Poisson (triangles) and Markov chain (circles) approaches. b) Delay of class-2 versus the throughput of class-2 for a twoclass flexible OCDMA network for three different approaches. 
In summary, this chapter presented a validation procedure carried out via numerical simulations, where the convergence of a flexible OCDMA network towards a conventional OCDMA network was assumed as the validation criteria. Numerical results have shown an excellent convergence, confirming therefore the robustness of the proposed analytical formalisms. Regarding the BER performance of flexible OCDMA networks, the numerical results showed that the BER equation based on the gaussian distribution underestimates the performance in unacceptable levels and incorrectly predicts the number of simultaneous users for any given BER value, which invalidates its use to assess the network performance. It also presented a packet throughput performance analysis of flexible OCDMA packet networks using separately Poisson, binomial, and Markov chain approaches for the composite packet arrivals distributions. On one hand, the Poisson-based approach proved to be a poor approximation as it incorrectly predicts the number of successfully received packets for most values of offered load, especially for user classes defined with high code weight. Even under favourable conditions, with a considerably large number of simultaneous users, the Poisson approach does not provide accurate results. On the other hand, the throughput performance evaluation based on the binomial approach proved to be more convenient and computationally faster than the one based on the Markov chain approach, which was assumed here as the benchmark. This is a consequence of simpler mathematical expression of the former that only depends on the packet correct probability and on the (binomial) probability distribution itself, whereas the Markov approach relies on a state transition matrix. In addition, the throughput performance evaluation based on the binomial approach proved to be just as accurate as the one obtained with the Markov approach (benchmark). Finally, numerical results have shown that only the packet delay evaluation based on the binomial (and also Markov) approach is accurate. 


\section{Chapter 5 \\ Polarization Chaos from Free- Running QW-VCSELs}

This chapter focuses on a practical solution based on deterministic chaos that can positively impact the level of security of optical networks at the physical layer. It consists of experimentally investigating the polarization chaotic dynamics of quantum-well vertical-cavity surface-emitting lasers (QW-VCSELs). We perceive this study as a prospective alternative for secure data transmission between a pair of synchronized QW-VCSELs, where the chaotic optical source discussed here is the primary kick-start of such a solution. In this manner, we start exploiting the complex dynamics of commercial QW-VCSELs which can be used not only for improving current solutions of secure data transmissions, but also for random number generation, cryptography and large-scale quantum networks. In the present setup, chaos is generated without any additional complexity such as optical feedback, parameter modulation or optical injection. Chaotic polarization dynamics have been originally observed in quantum-dot (QD)-VCSELs working in a free-running configuration and we reproduce here similar chaotic dynamics using a mechanical strain technique in QW-VCSELs.

We firstly introduce the features of chaos theory and its application in semiconductor laser systems, focusing on the polarization dynamics in QW-VCSELs. In this way, we describe both the experimental setup and how chaos dynamics is triggered by means of an external mechanical strain. A simple custom-made laser holder, used as part of the mechanical strain technique, guarantees that the investigated dynamical system can be easily reproduced in a cost-efficient way. Our experimental observations unambiguously confirm that polarization chaos can be successfully generated from a solitary QW-VCSEL operating in free-running configuration. This new finding represents a substantial contribution to the field of deterministic chaos in laser diode systems.

This chapter is organized as follows:

1) The key features and aspects of chaos theory and its connection to laser diode systems are briefly described in subsection 5.1.1, in which the low security level of current optical networks is one of the main motivations to pursue chaos communications. 


\section{PolarizATION CHAOS FROM FREe-RUNNING QW-VCSELS}

2) In subsection 5.1.2, two distinct semiconductor lasers are compared, namely QW-VCSEL and edge emitting laser (EEL), where QW-VCSELs have several advantages over EELs. Then, we conclude the introduction addressing polarization instabilities and chaos in VCSELs among with the description of double polarization switching (PS) events and the techniques frequently employed to trigger them described in subsection 5.1.3.

3) Subsequently, the experimental setup and the mechanical strain technique used during this study are thoroughly described in section 5.2.

4) Then, we finally analyse the complex polarization dynamics of QW-VCSELs in section 5.3, where the obtained dynamics are evaluated via LI-curves (subsection 5.3.1), radio frequency (RF)-spectra evolution (subsection 5.3.2), time-series and random like-hopping, largest Lyapunov exponent and GP algorithm (subsection 5.3.3).

5) Section 5.4 addresses the principal peculiarities in polarization dynamics between QW-VCSELs and QD-VCSELs, both operating in a free-running configuration, where comparisons are carried out in terms of polarization and frequency-resolved optical spectra (subsection 5.4.1) as well as orientation of the polarization modes (subsection 5.4.2).

6) We conclude this chapter with a summary and the principal perspectives and impacts of these new findings. 


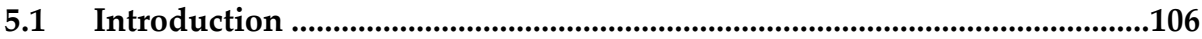

5.1.1 Chaos and its Potential for Secure Networks .........................................106

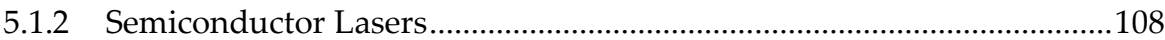

5.1.3 Polarization Instabilities and Chaos in VCSELs ....................................109

5.2 Experimental Setup and Mechanical Strain Technique...............................113

5.3 Experimental Chaos Identification ...............................................................116

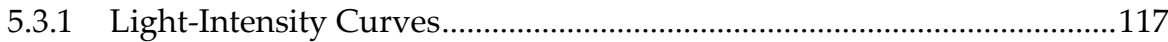

5.3.2 Radio-Frequency Spectra Evolution ......................................................119

5.3.3 Time-Series and Random Like-Hopping ..............................................120

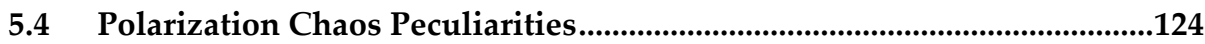

5.4.1 Light-Intensity Curves with an Iris Diaphragm ....................................124

5.4.2 Polarization and Frequency-Resolved Optical Spectra...........................128

5.4.3 Orientation of the Polarization Modes ...................................................130

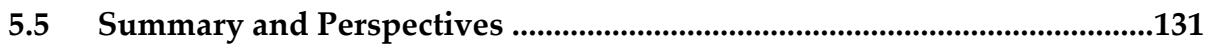




\subsection{Introduction}

We start this chapter by introducing general concepts on polarization chaos. We briefly discuss chaos theory and its concepts applied to laser diode dynamical systems, and the main differences between VCSELs and EELs, where we emphasize the intrinsic features of VCSELs that make them to stand out in today's optical systems. Finally, the last part of this chapter addresses deterministic polarization chaos from VCSELs: the system evolution towards chaotic dynamics is reviewed and the main characteristic features of the polarization chaos dynamics are summarized. Finally, it is worth mentioning that chaotic polarization dynamics have been originally observed in QD-VCSELs [116], where double polarization switching events, a random-like hopping between two elliptically polarized states and reduction of the residence time for increasing injection current were reported. We investigate here these features also for QW-VCSELs.

\subsubsection{Chaos and its Potential for Secure Networks}

The current research on complex behavior of dynamical systems span fields ranging from physics to economics. This only became possible thanks to the studies on weather prediction carried out by E. Lorenz that culminated with the Lorenz attractor's [117]. His findings on weather prediction helped accidentally to pave the way towards the foundation of chaos theory and the description of nonlinear dynamical systems. The deterministic, nonlinear and complex behavior observed in dynamical systems was later denominated chaos and is especially characterized by means of its high sensitivity to initial conditions. For example, let us consider two systems that start evolving with slightly minor differences in their initial conditions, but still virtually indistinguishable from each other. These systems will eventually exhibit completely different evolutions after a certain time with widely diverging trajectories and outcomes. This happens even though these systems are deterministic, which means that their future behavior can be fully determined by their initial conditions, with no random elements involved, but they are still unpredictable [117]. Accordingly, this event or behavior is known as chaos.

Chaos theory accounts for deterministic systems whose behavior cannot be predicted during their long-term evolution, but whose evolution is mathematically defined. Deterministic chaos and chaotic systems, have been explored and used for a large range of applications, from weather and solar systems until traffic forecasting, population growth models of different species, and economics as well as finance models, to name but a few [118]. Despite many viable solutions and the potential for even more chaos-based applications, we are particularly interested in secure chaos communication. 
This type of application has become of great interest in communication networks and relies on the use of deterministic chaotic dynamics for security purposes, where the main goal is to provide network users with higher security levels at the physical layer so that the transmitted data information remains safe from possible eavesdroppers. As such, deterministic chaos constitutes the basis of chaos communication, where the dynamic and non-linear properties of chaotic systems are exploited to wisely hide users' data information transmitted through a network. Effective security improvements have become an essential requirement not only for optical access networks, and consequently OCDMA-based systems, but also for the future generation optical networks. In this sense, chaos communication can be regarded as a prospective solution to provide a robust platform with a considerable higher level of security at the physical layer for optical networking.

Chaos communication has been considered for several different network scenarios, being such as wireless networks [119], free space optical systems [120] and mainly for optical networks [121], [122]. For example, its use in wireless networks is attractive due to some well-known shortcomings of the wireless environment such as multipath fading and interferences and also because of its simple generation of chaotic signals via low-cost, low-power consumption, and easily integrated devices [119]. However, this is no longer true for optical networks, where the optical source for chaos generation is not as developed and matured as in wireless networks. To date, none of the proposed chaos-based schemes have demonstrated a cost-effective optical chaotic source that supports chaos generation without any external apparatus (feedback, modulation, injection, etc). In this way, this chapter proposes a commercial low-cost optical chaotic generator working in a free-running configuration that might be useful for chaotic networks, where the original user's information is retrieved via a synchronization technique.

Chaos synchronization [123], [124] is an interesting event used in chaos communication that can be achieved via different methods, where synchronization occurs when two chaotic oscillators such as semiconductor lasers are coupled. Chaos communication uses a chaotic signal to modulate a data signal followed by a synchronization scheme between a chaotic transmitter and receiver.

Several schemes for synchronization of semiconductor lasers have been proposed or experimentally demonstrated for this task [125], [126] and [127], but the current lack of practical and cost-effective solutions restricts their widespread practical applicability. Indeed, external apparatus, like for example feedback, adds substantial complexity to the system and significantly hinders its widespread use. In contrast, the recent demonstration of chaos generation from a free-running quantum-dot (QD)-VCSEL [116] has motivated the investigation of such dynamics also from solitary QW-VCSELs. In this way, this chapter investigates the potential of commercial QW-VCSELs in generating deterministic chaos. 


\subsubsection{Semiconductor Lasers}

Semiconductor lasers have been subjected to constant improvements during the last decades and their performances have considerably increased in this period. They are based on semiconductor gain media and are suitable for a wide range of applications which include optical readers as well as mouses, laser printing and especially optical communication purposes, to name but a few. From the several types of semiconductor lasers currently available, VCSELs have recently become one of the most common types due to advantages such as emission perpendicular to the wafer plane over another common type of semiconductor optical source, known as edge emitting lasers (EELs). VCSEL-based solutions have been developed for a wide field of applications ranging from data centers to optical access networks, covering wavebands from $850 \mathrm{~nm}$ to $1555 \mathrm{~nm}$.

Although both VCSELs and EELs can be used for multiple purposes, they do present structural differences that make VCSELs to stand out as the most paramount one, mainly due to their unique planar structure with vertical light emission rather than the horizontal emission of EELs. This structural difference is depicted in Figure 5.1, where the light beam emission of VCSELs is perpendicular to the active layer (plane) rather than to the horizontal plane as in EELs [128]. In other words, the light travels perpendicularly to the wafer and is emitted from the surface in VCSELs. Furthermore, EELs have an elliptical light output and eventually require more optical components to focus the output beam. Because of their lateral emission, EELs based solutions are vulnerable to catastrophic failure since whenever an EEL fails, the entire system fails. Remarkably, the smaller active region of VCSELs demands a lower total injection current which, by its turn, allows lower current threshold, which can be as low as $0.5 \mathrm{~mA}$. VCSELs also have low-divergence that renders their beam symmetrical and nearly circularly uniform with no astigmatism.

Furthermore, VCSELs can be tested still at the wafer stage, resulting in a more controlled and flexible manufacturing process with lower costs when compared to other technologies. Because VCSELs emit light perpendicular to the surface of the wafer as seen in Figure 5.1, hundreds of individual VCSELs can be processed on a single wafer either as one or two-dimensional arrays to scale power output to optimally match specific application requirements. VCSELs have low parasitic and are thermally efficient, providing better heat dissipation than EELs for the same injection power. 


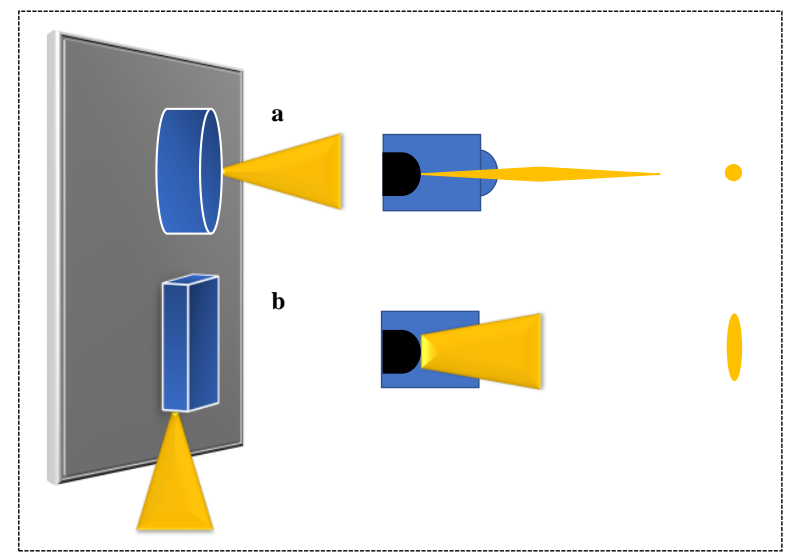

Figure 5.1. Schematic designs of classical VCSEL and edge-emitting laser structures. a) Scheme of a VCSEL with emission perpendicular to the wafer plane and with an output beam on the vertical axis. b) Scheme of an EEL structure with emission perpendicular to the wafer plane on the side of the structure. Figure adapted from [128].

\subsubsection{Polarization Instabilities and Chaos in VCSELs}

VCSELs are widely used in communication systems as optical sources, where they have the role of providing a carrier signal that will be modulated with the information to be transmitted over a network. However, small perturbations in the physical structure of VCSELs might lead to internal instabilities and give rise to complex dynamics that are not part of their regular operational behaviour. Those instabilities can be regarded as a drawback for polarization sensitive applications and traditional coherent optical communication (where phase and polarization of a signal should be controlled). In addition, small perturbations such as changing the substrate temperature or the injection current might be sufficient to trigger unexpected dynamics in VCSELs.

For example, polarization instabilities along with polarization switching (PS) events might be triggered via those small perturbations, mainly because they can be easily modified in practice. In this way, the first LP eigenmode, otherwise stable at threshold, is destabilized and after some complex dynamical transitions, the laser settles on the orthogonal LP mode. In this event, the two orthogonal LP eigenmodes are normally detuned due to the laser cavity birefringence, and are defined as lowfrequency (LF) and high-frequency (HF) eigenmodes. Accordingly, two different types of polarization switchings can be identified, namely, from HF to LF mode (defined as type I) and a switching from LF to HF mode (defined as type II) [117]. 


\section{POLARIZATION CHAOS FROM FREe-RUNNING QW-VCSELS}

When a specific combination of both switchings happens, this event is identified as a double PS, in which initially a type I switching occurs followed by a type II PS [129]. These different types of PS are shown in Figure 5.2 [130], where the polarization resolved output power for different substrate temperatures is plotted for three distinct situations. A single PS defined as type I (from HF to LF mode) happens for low temperatures $\left(\mathrm{T}=10^{\circ} \mathrm{C}\right)$, where the emitting laser device experiences an abrupt switching between the two orthogonal LP modes as seen in Figure 5.2a. In contrast, for high temperatures $\left(\mathrm{T}=55^{\circ} \mathrm{C}\right)$, a single PS defined as type II (from LF to HF mode) happens, where an abrupt switching of the higher frequency mode happens for values of high injection current as seen in Figure 5.2c. Finally, a scenario of a double PS event with PS of type I followed by a PS of type II is observed in Figure $5.2 \mathrm{~b}$ for an average substrate temperature $\left(\mathrm{T}=15^{\circ} \mathrm{C}\right)$.
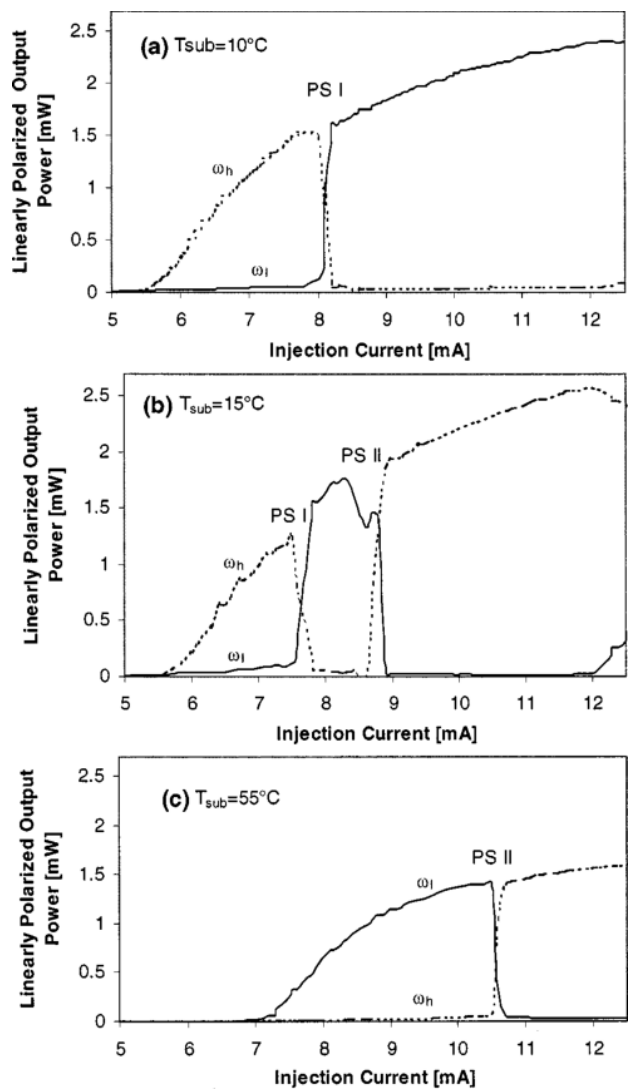

Figure 5.2. Distinct types of polarization switching events. Polarization-resolved optical output power versus the injection current for three different substrate temperatures. a) $\mathrm{T}=10^{\circ} \mathrm{C}$ featuring a PS of type I, b) $\mathrm{T}=15^{\circ} \mathrm{C}$ featuring a double PS event, type I followed by type II, (c) T $=55^{\circ} \mathrm{C}$ featuring a PS of type II. Picture taken from [130]. 
Besides the possibility of triggering unexpected polarization dynamics in VCSELs by changing the temperature or injection current, techniques based on mechanical strain have also been reported as an efficient way of triggering PS events. One of the first approaches to use strain in laser diodes was suggested in [131] and [132], where they highlighted the role of applied strain to trigger polarization instabilities. A controlled amount of anisotropy was applied onto a VCSEL via a technique called hot-spot, where an external laser beam is used to heat the surface near a VCSEL creating thermal expansion. This expansion induces strain, which by its turn changes the birefringence of the gain medium. The magnitude of the birefringence variation can be tuned in a continuous and reversible way by varying the applied heating power. Accordingly, depending on the orientation and on the strength of the applied strain, the anisotropy of the cavity is changed, hence leading to polarization instabilities.

Alternatively, a mechanical stress technique has also been used to change the internal dynamics to induce anisotropic variations in the cavity of a QW-VCSEL in [129]. This technique proved useful in achieving switching between two LP modes via birefringence and gain anisotropy. More recently, a similar strain technique has been developed to demonstrate the possibility of overcoming the limitation of relaxation oscillation frequency in single-mode VCSELs, where oscillations and birefringence splitting above $40 \mathrm{GHz}$, and potentially up to $250 \mathrm{GHz}$, have been experimentally demonstrated [133], [134]. In this experiment, a considerably high level of mechanical stress was applied onto a VCSEL so that the birefringence could be maximised. The birefringence tuning in the laser cavity led to a large frequency difference between the two linearly polarised states of the VCSEL.

Finally, we discuss the central focus of this chapter: polarization chaos. As already mentioned earlier, polarization chaos has only been observed in QD-VCSELs, but excellent agreement was obtained with standard models for VCSELs hence suggesting that the same dynamics could be obtained in commercial quantum-well devices. Indeed, this point is the main motivation for this work: using strain to unlock polarization chaos in quantum well VCSELs.

Typically, polarization chaos appears in the scenario shown in Figure 5.3, which was taken from [130]. At threshold, the laser emits linearly polarized (LP) light as normally expected. Then, a transition towards an elliptically polarized (EP) state occurs (at $\mathrm{I}_{\mathrm{A}}$ in Figure 5.3a) followed by the emergence of random-like hopping dynamics between two distinct EP states (between Is and $I_{R}$ in Figure 5.3a). In practice, using a polarizer oriented at $45^{\circ}$ from the LP stable at threshold to discriminate between the two EP states, the temporal dynamic looks like the one shown in the inset of Figure 5.3b: seemingly random jumps between two distinct levels. Interestingly, the time between two successive jumps - also called dwell-time - varies significantly as the current increases. 
a

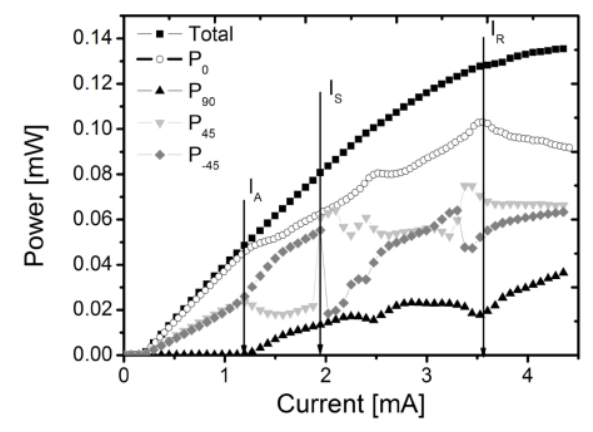

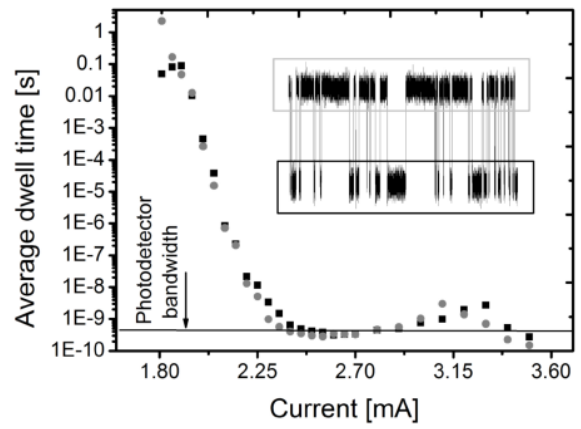

Figure 5.3. Polarization resolved LI-curves for increasing currents and statistics of the mode hopping dynamics. a) Polarization resolved-LI curve for increasing injection currents. The laser emits on a LP state from threshold to $\mathrm{I}_{\mathrm{A}}$ and then on the EP state for $\mathrm{I}_{\mathrm{A}}<\mathrm{I}<\mathrm{Is}$. Random like hopping dynamics between two EP states are observed for Is $<\mathrm{I}<\mathrm{IR}$, and the laser emits in a twomode emission for I > IR. b) Evolution of the average dwell-time for the mode-hopping dynamics with an inset showing the time-series of the random-like hopping for polarization projection at $45^{\circ}$ concerning the linear polarization at threshold. Pictures taken from [138].

Indeed, as displayed in Figure 5.3b, the jump speed goes from second to nanosecond time-scale. This is however only one part of the overall more complex chaotic behaviour: besides the mode-hopping dynamics, fast oscillations, typically around 8 to $10 \mathrm{GHz}$, are also observed. It can be better understood by considering the doublescroll shape of the chaotic attractor (which reminds the famous Lorenz' attractor): the fast oscillations correspond to the rotation around the scrolls while the hopping dynamics appear as the system goes from one scroll to the other. Finally, at higher currents (above IR in Figure 5.3a), chaos disappears and the laser emits in a two-mode emission that has been linked to a quasi-periodic behaviour [135].

In the end, it is important to remark that this whole behaviour has been successfully explained theoretically as dynamics accompanying a type II switching [135]: in the case shown in Figure 5.3, the laser never settles on the LP orthogonal to the one stable at threshold simply because the switching point is expected to occur at unrealistic level of currents. The context of a type II switching seems however strictly required to generate polarization chaos. 


\subsection{Experimental Setup and Mechanical Strain Technique}

We consider the illustration of the experimental setup and a schematic representation in Figure 5.4 and Figure 5.5, respectively. In fact, two different setup configurations are considered. First, classical measurements are carried out using the scheme shown in Figure 5.5a, where the first mirror (M1) is down and the output beam from the VCSEL reaches the multimode fiber depicted in Figure 5.5a (dotted line). Then, a more complex setup is used to carry out measurements addressing the influence of a second higher-order transverse mode using a nearly closed iris diaphragm to filter out the mode. To this aim, the beam output of the VCSEL reaches mirror \#1 and \#2 and is coupled into the multimode fiber depicted in Figure $5.5 \mathrm{~b}$ (dashed line). In this configuration, two lenses (L2 and L3) are used to extend the light beam so that the higher-order mode can be spatially filtered out. Another lens (L4) is placed before the objective element. A CCD camera (shown in Figure 5.4) is used to capture the spatial beam profile of the VCSEL output with the mirror \#2 down.

Initially, the laser beam from the QW-VCSEL is collimated by an aspherical lens coupled to a multimode fiber. To avoid unintentional reflections from the fiber frontfacet back into the laser cavity, we use an optical isolator followed by a half-wave plate used to select the polarization direction that will be analysed. Both the optical isolator and the half-wave plate are placed between the laser and the fiber front-facet as illustrated in Figure 5.4. The $0^{\circ}$ reference is defined as the direction of the linearly polarized light emitted at lasing threshold when no stress is applied. As a result, the two preferred polarization axes, the so-called polarization eigenmodes of the laser, correspond to the polarization at $0^{\circ}$ and $90^{\circ}$ respectively.

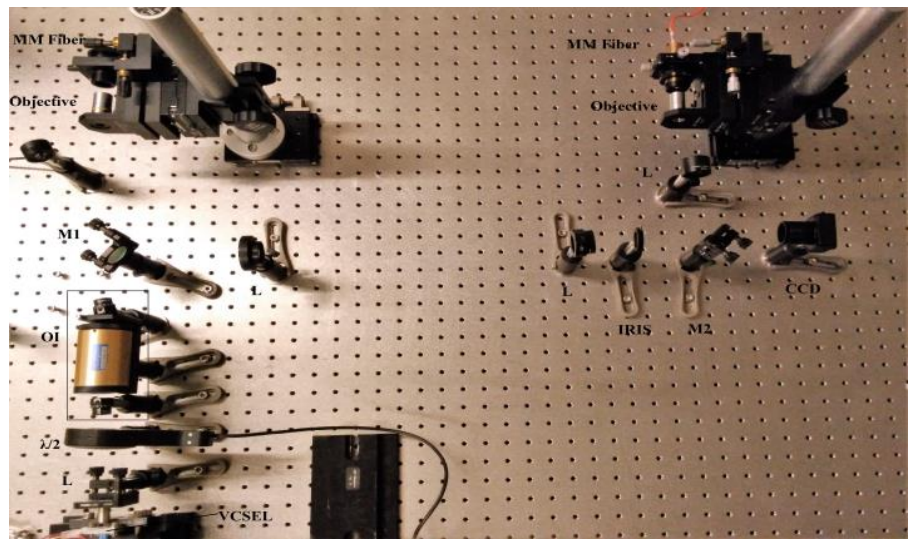

Figure 5.4. Picture of the full experimental setup used to exploit the complex polarization dynamics of a QW-VCSEL subjected to mechanical stress. L: lens, $\lambda / 2$ : half wave plate, OI: optical isolator, M1: mirror \#1, MM Fiber: multimode fiber, IRIS: iris diaphragm, M2: mirror \#2, CCD camera, objective and VCSEL. 


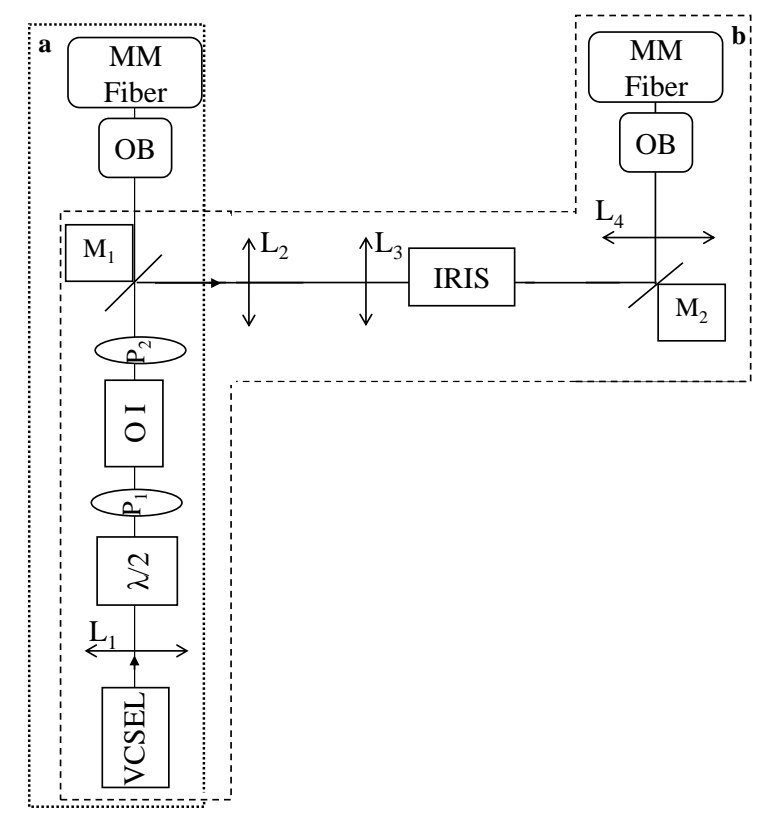

Figure 5.5. Schematic of the experimental setup used to exploit the complex polarization dynamics of a QW-VCSEL subjected to mechanical stress. The full line represents the light path starting from the QW-VCSEL until reaches one of the two-possible multimode fiber configuration. a) setup configuration used for general characterization of the QW-VCSEL with the mirror (M1) down (dotted line). b) setup configuration used to exploit the influence of the second higher-order mode (dashed line). L1: lens \#1, $\lambda / 2$ : half wave plate, L2: lens \#2, OI: optical isolator, L3: lens \#3, M1: mirror \#1, L4: lens \#4, OB: Objective, MMFiber: multimode fiber, IRIS: iris diaphragm, M2: mirror \#2 and VCSEL.

We employ a commercial single-mode QW-VCSEL (ULM850-PM-TN-S46XZP from Philips Photonics) in a TO46 package, which was properly de-capsulated, emitting at $850 \mathrm{~nm}$ with lasing threshold around $0.5 \mathrm{~mA}$ and stabilized at a temperature of $22^{\circ} \mathrm{C}$. The QW-VCSEL needs to be de-capsulated so that the mechanical stress can be applied onto it. Both static and dynamic polarization-resolved measurements have been carried out to investigate and evaluate the polarization dynamics of the QWVCSEL, in which for the former measurement a power meter and an optical spectrum analyser (OSA) (ANDO AQ6317) are used, while for the latter a high-speed photodetector (NewFocus 1554-B, $12 \mathrm{GHz}$ ), a wide bandwidth electronic amplifier (20 GHz), an electrical spectrum analyser (Anritsu, MS2667C, $30 \mathrm{GHz}$ ) and an oscilloscope (Tektronix CSA7404, 4 GHz, 20 GSamples/s) are used. Initially, the setup also contained a Fabry-Perot Interferometer (FPI) with a $10 \mathrm{GHz}$ free spectral range. However, it was not possible to retrieve any signal from the extinct mode due to the limited sensitivity of the FPI. 
Next, Figure 5.6 illustrates the custom-made holder fabricated with the purpose of applying stress onto the QW-VCSEL. The holder consists of a main aluminium plate and a lid (cover) that along with two screws fix the QW-VCSEL to the main plate. A steel rod placed between the metal plate and the lid load mechanical stress onto the de-capsulated QW-VCSEL. This lid is bent by both the steel rod and the screws as the level of mechanical stress increases, as depicted in Figure 5.6. Fastening or loosening these screws that fix the lid allows one to control the level of applied stress [129].

A thermistor is placed inside the holder via a lateral hole. A Peltier element (not shown in the figure) is glued on to the back of the rectangular main plate so that the laser device temperature can be controlled with the help of a thermistor. The suggested dimensions and details of the holder structure are illustrated in Figure 5.7, where standard screw thread dimensions are used. The low-cost holder made of aluminium is simple to be reproduced, which renders the experimental setup easily reproducible with off-the-shelf components (QW-VCSEL, polarizer, optical isolator, and multi-mode fiber). Noteworthy, a QW-VCSEL normally emits linearly polarized light with direction related to the crystallographic axes of its structure when operating at the biasing threshold [117]. However, inducing stress onto a VCSEL can most likely lead to complex polarization instabilities. In this way, we demonstrate in this chapter that the technique developed here can be used to successfully trigger polarization chaos from an off-the-shelf QW-VCSEL device working in a free-running configuration without the additional complexity of optical feedback or other external forcing.

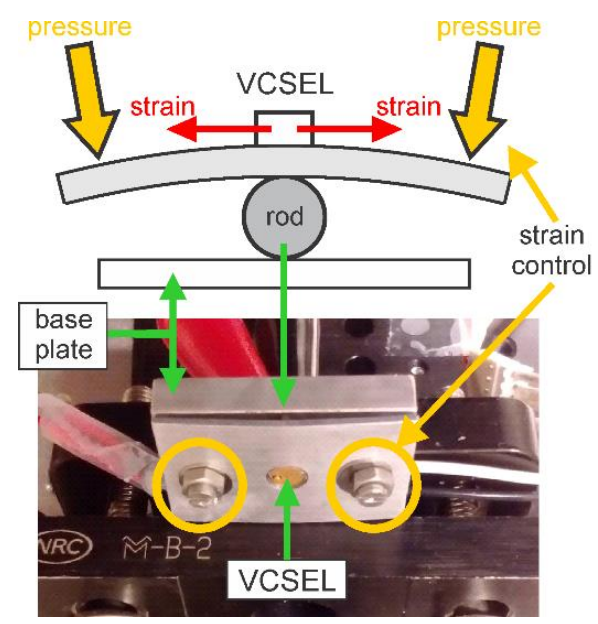

Figure 5.6. Custom-made holder based on the mechanical stress technique. The holder comprises an aluminium plate with a central hole to place the already packaged QW-VCSEL in combination with a pressing plate fixed with two screws used as a lid. By placing a steel rod behind the laser package, the pressure applied by the pressing plate induces anisotropic strains in the device cavity. 


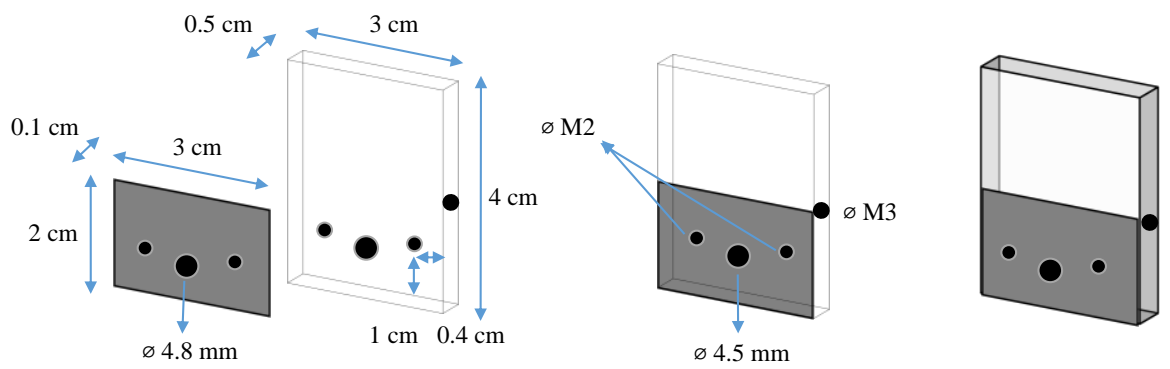

Figure 5.7. Schematic design and suggested dimensions of the custom-made VCSEL holder for the application of the mechanical stress technique. The packaged VCSEL is placed behind the central hole of the lid and a steal rod (not illustrated) is placed between the VCSEL and the metal plate. Pressure can then be applied on the metal lid and adjusted using the M2 screws (not illustrated) on each side of the device. A thermistor is placed into the metal plate's right-side hole to control the device temperature.

\subsection{Experimental Chaos Identification}

In this subsection, we show via the experimental data recorded from several measurements, the dynamical features observed from QW-VCSELs subjected to mechanical stress. We consider the experimental setup described in the previous section. In this way, we start with standard measurements such as polarization resolved LI-curves, where polarization switching can be easily confirmed. Then, we measure the radio-frequency spectra evolution of the dynamics as well as the time series of the polarization resolved output power.

It is worth pointing out that all measurements were not carried out on the same day due to time constraints, which can eventually lead to minor variations among them. The following common features and conditions are assumed: a) all measurements consider an increase of injection current; $b$ ) all measurements are carried out at room temperature around $23^{\circ}$; c) the QW-VCSEL is stabilized at $22^{\circ}$; d) the axis of the linearly polarized light at lasing threshold for polarization orientation at $0^{\circ}$ is considered as a reference; e) the same level of stress applied onto the investigated QW-VCSEL is considered for all measurements. The measurements have been carried out for four different QW-VCSEL devices and equivalent results have been obtained. 


\subsubsection{Light-Intensity Curves}

Among all possible dynamical and complex fluctuations that might be observed in VCSELs, PS is usually one of the main instabilities and can be attributed to the competition between two preferred LP eigenmodes. Theoretically, polarization chaos has been linked with PS events of type II, i.e. switching from a lower frequency to a higher-frequency eigenmode [135]. In addition, the transition from LP to elliptically polarized (EP) states might also suggest chaotic dynamics. Even though we are not able to measure the frequency splitting between the two polarization eigenmodes due to the limited resolution of the OSA, and thus not able to experimentally discriminate type I and type II PS events, we still look for PS events as a first indicator of potential interest. Using the custom-made holder and the mechanical stress technique previously described, it might be possible to change the anisotropy of the cavity depending on the amount of applied stress, leading to polarization instabilities and even chaotic fluctuations.

It is worth mentioning that the amount of applied stress is important in the process of triggering polarization dynamics. In fact, we stablished our own stress scale with 9 distinct levels, where for each level the screws are fastened by one quarter turn. Interesting polarization dynamics are seen from the stress level 6 onwards and no PS events are observed for the first levels of stress. For example, the polarization resolved light-intensity (LI) curves for increasing injection current and for stress levels \#3 and \#5 are plotted in Figure 5.8.

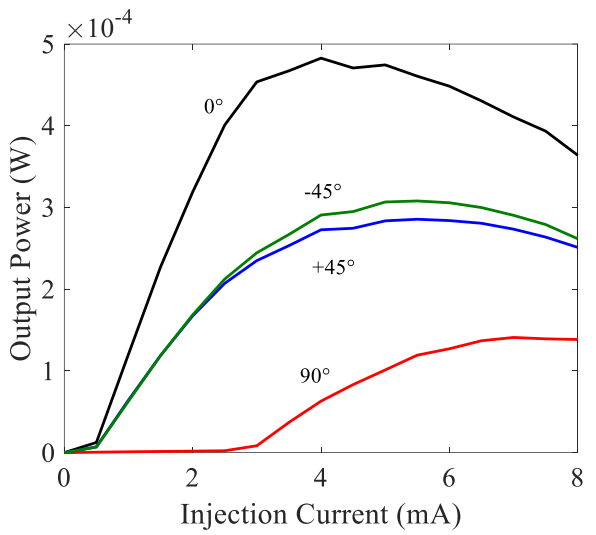

(a)

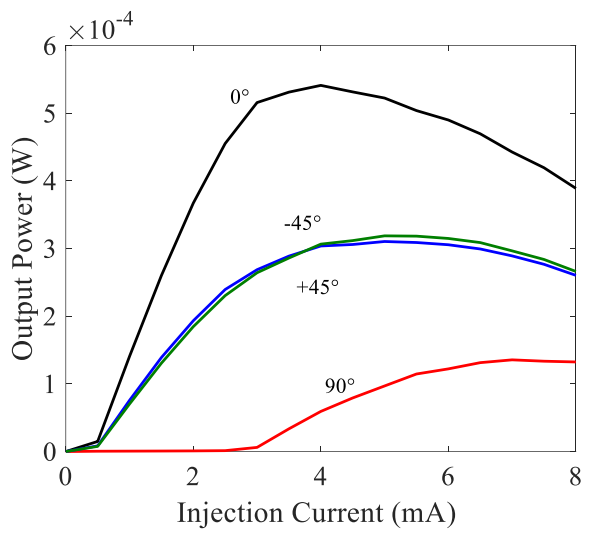

(b)

Figure 5.8. Polarization resolved LI-curves for increasing injection current and for polarization projection at $0^{\circ}, 90^{\circ}, 45^{\circ}$ and $-45^{\circ}$ with respect to the LP at threshold. No PS events are observed. a) Stress level \#3, and b) stress level \#5. 


\section{POLARIZATION CHAOS FROM FREE-RUNNING QW-VCSELS}

As can be observed, the polarization behaviour is very similar for both levels of stress with no PS events happening. In contrast, when the amount of applied stress is gradually increased, the laser dynamics is destabilized and we successfully achieve a double PS event as shown in Figure 5.9, which shows the polarization resolved-LI curves for increasing injection current. Even though it is not possible to quantify the amount of stress loaded onto the investigated QW-VCSEL with the technique developed here, we are able to say that a high level of applied stress such as \#8 and \#9 from our scale was used for the results in Figure 5.9. Remarkably, the higher level of stress is responsible for the displacement of the double PS event to regions of larger injection current. This effect is clearly noted for the first PS event, where it happens around $1.8 \mathrm{~mA}$ for stress level \#8 (see Figure 5.9a), and around $3.3 \mathrm{~mA}$ for stress level \#9 (see Figure $5.9 b$ ).

Normally, double PS events are observed as type I followed by type II [130], [136], which suggests that important dynamic fluctuations can be expected after the second PS. Accordingly, notice that the laser starts emitting LP light along with the $0^{\circ}$ and $90^{\circ}$ polarization directions, i.e. at threshold the laser emits from the orthogonal eigenmode (at $90^{\circ}$ ) compared to the case without stress. A PS event happens at around $1.6 \mathrm{~mA}$ and the measured output power for polarization at $90^{\circ}$ is extinct, see Figure 5.9a. However, the output power for polarization at $0^{\circ}$ changes abruptly to 0.4 $\mathrm{mW}$, characterizing the first PS event as type I according to the literature.

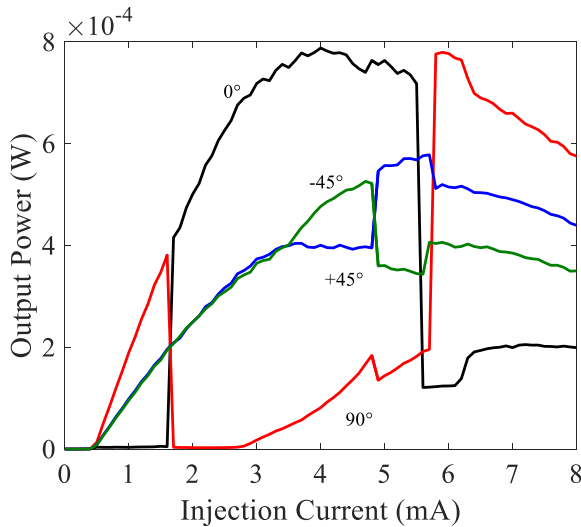

(a)

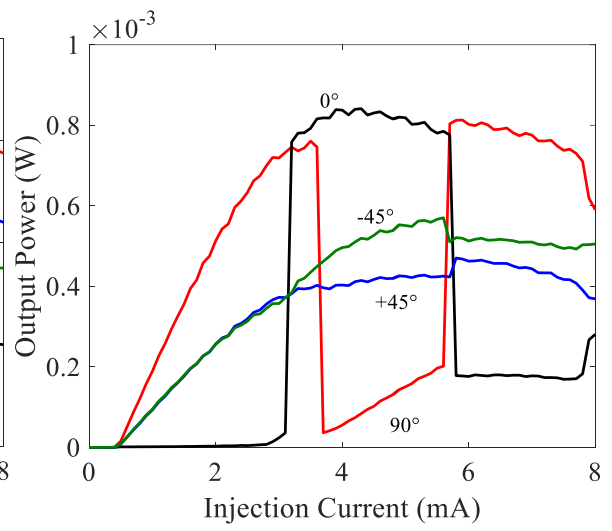

(b)

Figure 5.9. Polarization resolved LI-curves for increasing injection current and for polarization projection at $0^{\circ}, 90^{\circ}, 45^{\circ}$ and $-45^{\circ}$ with respect to the LP at threshold. A double PS event can be observed in both pictures. a) A medium level of mechanical stress (\#8) is loaded onto the QWVCSEL. b) A high level of stress (\#9) is loaded onto the QW-VCSEL. 
Finally, a second PS appears establishing the double PS event for an injection current above $6.2 \mathrm{~mA}$ accompanied by polarization hopping dynamics, see Figure 5.9a. This eventually might happen because of anisotropic variations of the laser cavity, which by its turn is caused by the high level of applied stress but with gain saturation also influencing the laser dynamics. The second PS event appears at a higher level of injection current (>6 mA), which is more than 12 times the threshold current since the biasing threshold current is $0.50 \mathrm{~mA}$.

\subsubsection{Radio-Frequency Spectra Evolution}

Normally, double polarization switching is reported as type I followed by type II [130], which suggests that interesting dynamical behaviour could be expected after the second PS. Accordingly, we notice that the laser starts emitting LP light along with the $90^{\circ}$ polarization direction, i.e. at threshold the laser emits from the orthogonal eigenmode (at $90^{\circ}$ ) compared to the case without applied strain. Then, a PS happens and the measured power at $90^{\circ}$ is extinct whereas the power at $0^{\circ}$ changes substantially, characterizing the first PS event as type I with a second PS appearing for a higher injection. Since type II PS events have been associated with polarization dynamics, we perform dynamic measurements especially focusing on an injection current level that places us just after the second PS event.

The results relative to the dynamic measurements are shown in Figure 5.10, where the polarization resolved LI resolved radio frequency (RF) spectra evolution for increasing injection current is plotted. Panels $a$ and $b$ in Figure 5.10 show the evolution of the RF spectra recorded for the polarization at $0^{\circ}$ and $45^{\circ}$ when increasing the injection current. At high currents, only a single peak appears for the polarization at $45^{\circ}$, hence suggesting a behaviour close to a periodic solution with a frequency around $8 \mathrm{GHz}$. A sudden frequency change of about $600 \mathrm{MHz}$ appears at the onset of the second switching that clearly resembles the limit cycle bistability in chaotic QDVCSELs [137].

In contrast, although the same peak appears for the polarization at $0^{\circ}$ as displayed on panel a, we also observe the emergence of a featureless part in the low frequency range (below $1 \mathrm{GHz}$ ) as highlighted in the zoomed-in plot of panel $c$. This result suggests a relatively slow dynamic behaviour in this current range, which appears on top of the oscillations around $8 \mathrm{GHz}$. The lack of any features indicates that the corresponding dynamic behaviour does not exhibit any characteristic frequency, hence suggesting a chaotic behaviour. 


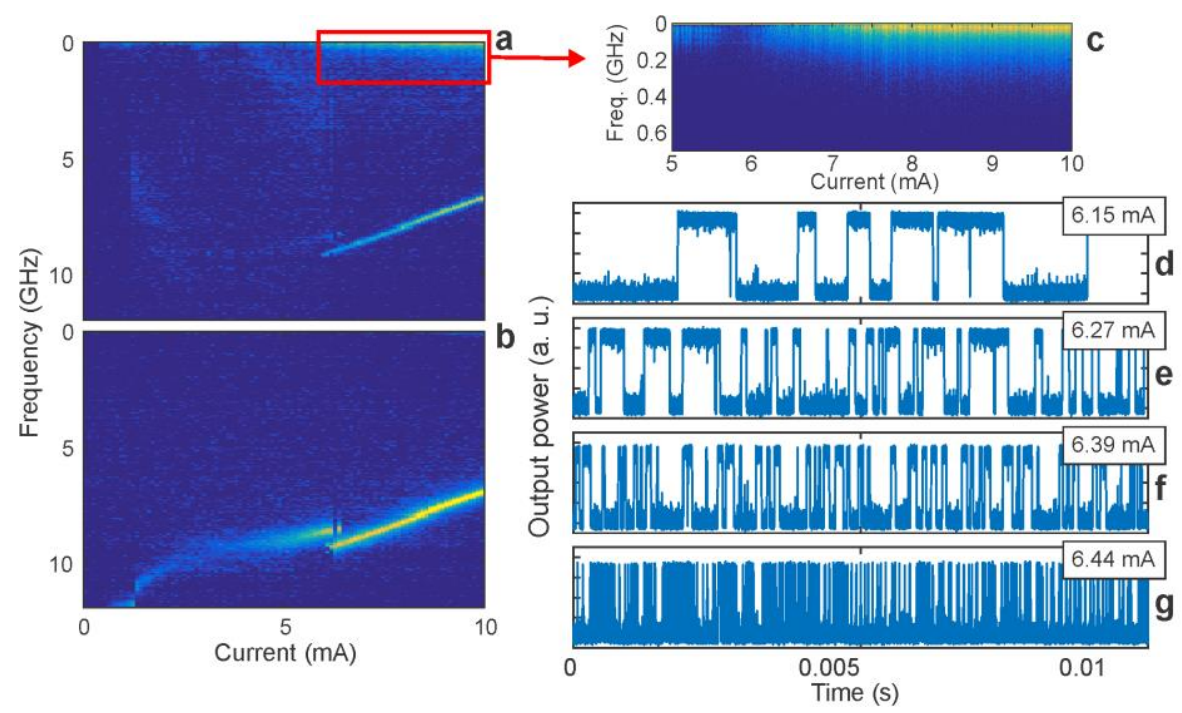

Figure 5.10. Dynamic measurements. Evolution of the Radio Frequency spectrum for increasing injection current for polarization at $0^{\circ}$ a) and at $45^{\circ}$ b). Panel c) shows the zoomed-in plot highlighted by the red rectangle in (a) showing the featureless low-frequency contribution corresponding to the mode-hopping dynamics. In d) to g), we show time-series recorded for polarization at $90^{\circ}$ showing random-like hopping between polarization eigenmodes with a significant decrease of residence time for increasing injection current at: 6.15, 6.27, 6.39 and 6.44 $\mathrm{mA}$ respectively.

\subsubsection{Time-Series and Random Like-Hopping}

We now carry out dynamical measurements focusing on current levels around the second PS event observed in the LI-curves since type II PS events have been associated with polarization dynamics. The results relative to the dynamic measurements are shown and investigated in this subsection. The time-series for projection at $0^{\circ}$ with different values of injection current is plotted in Figure 5.10, panels (d), (e), (f) and (g). One can clearly note random-like hopping dynamics between two states, which occurs in a similar fashion as previously observed for QDVCSELs [116]. Nonetheless, it is worth noting that the eigenmode hopping dynamics we obtained is significantly slower than the one reported in QD-VCSELs [138].

While an average residence time - i.e. the time between two successive jumps - below the nanosecond has been reported for QD-VCSELs [138], here the average residence time hardly reaches the microsecond time scale. The evolution of the residence time for increasing currents remains however similar as it exhibits an exponential decrease 
as the injection current is increased, as opposed to the evolution observed for stochastic mode hopping [139]. Observe that the random-like hopping between the upper and lower levels is asymmetric, with the system staying on average longer in one level than the other.

Next, we carry out numerical investigations using the experimental time-series data to fully confirm the deterministic nature of the polarization dynamics, as also developed in [116], [117]. We start evaluating a rough estimate of the average residence time - also called dwell-time. The dataset is relatively small and thus we limit our analysis to an apparent trend. Then, Figure 5.11 shows the average residence time. The global trend for the average dwell time is clear as shown in panel (a), where a good fit is obtained with a linear approximation. This outcome confirms the exponential decrease of the residence time as the current is increased. The results shown in (b) are obtained considering separately the lower and upper levels. We can clearly observe a strong gap between the two set of data as a difference of about 2 orders of magnitude is recorded between the residence times of the two levels. Strong fluctuations are also observed but, again, the trend is clear and suggests an asymmetrical behavior as analyzed in [140]. Finally, in the last plot we show the number of jumps versus injection current, which is the number of jumps in the recorded time-series. For low current values, the number of jumps is small but for current levels above $7.5 \mathrm{~mA}$, hundreds of jumps per point have been recorded.
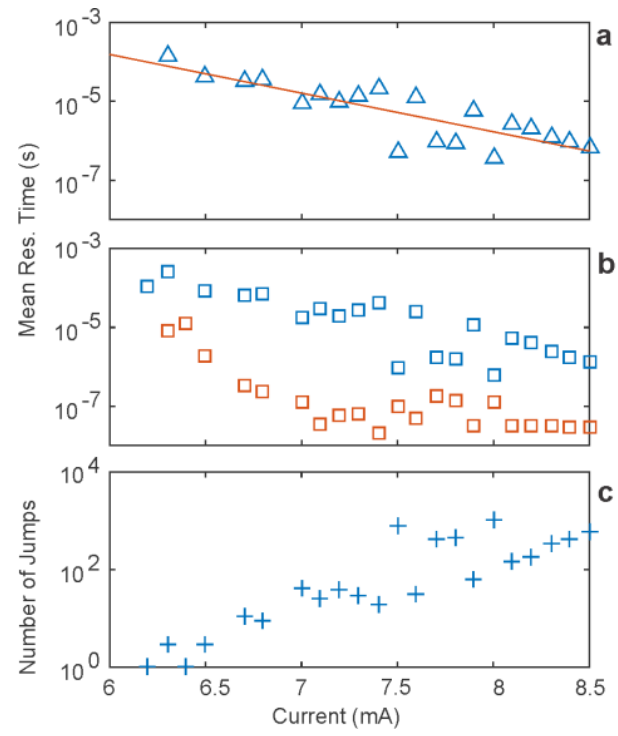

Figure 5.11. Mode hopping statistics. a) Average residence time when considering all jumps. The red line gives a linear fit. b) Average residence time for the upper (red) and lower (blue) levels. The upper and lower levels are defined for a time-series recorded at $0^{\circ}$ polarization. c) Number of jumps considered for each point. 
Now we use the so-called Wolf's algorithm [141] to estimate the largest Lyapunov exponent (LLE) from experimental time-series to confirm that the dynamics exhibit a non-zero but finite value of the LLE. The LLE characterizes how fast two nearby trajectories diverge in the system phase space. Thus, from a theoretical point of view: chaotic systems exhibit a finite positive (non-zero) LLE, a purely stochastic process has an infinite LLE, and a stable process has a negative LLE. We show the evolution of the estimated LLE for increasing injection current in Figure 5.12. Although very low LLEs are obtained at low current values, we observe a clear increase at higher injection currents.

As discussed in [142], the complexity of the dynamics mostly arises from the jumps between the two scrolls of the chaotic attractor. Thus, we observe a clear correlation between the estimated LLE value and the average residence time. Overall, the use of Wolf's algorithm clearly yields a finite non-zero value of the Largest Lyapunov exponent coherent with the chaotic interpretation of the dynamics.

Next, we use the so-called Grassberger-Procaccia (GP) algorithm to estimate the Kolmogorov entropy $\left(K_{2}\right)$ from the time-series data, and also an estimate of the correlation dimension of the investigated chaos, if the value of the $K_{2}$-entropy converges [117]. The same approach and notations from [116] are used, including the re-embedding procedure introduced in [143] before processing the experimental data with the GP algorithm. Hence, the results of the GP algorithm are shown in Figure 5.13 , in which the $K_{2}$-entropy converges along with the correlation dimension $D_{2}$. The GP algorithm yields a non-zero but finite estimate of the $K_{2}$-entropy $\left(K_{2} \approx 5.210^{-3} \mathrm{~ns}^{-1}\right)$ with a corresponding correlation dimension $D_{2} \approx 2.04$. The Kolmogorov entropy is strictly positive, which confirms the chaotic nature of the dynamics and both results further confirm that the reported dynamics is fully deterministic.

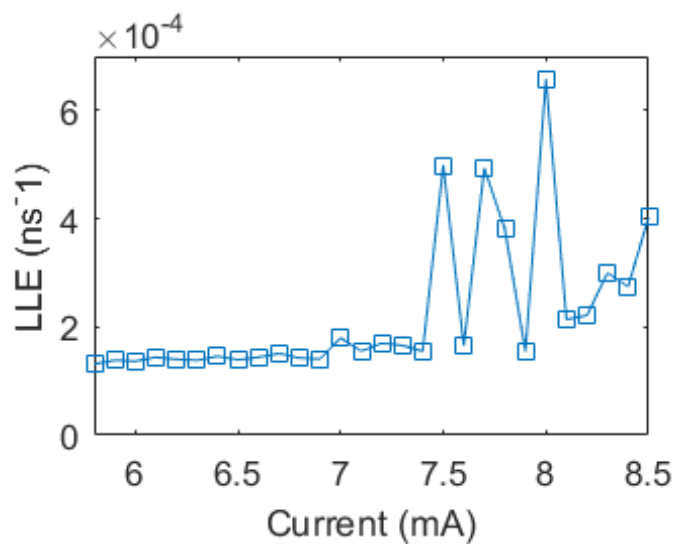

Figure 5.12. Estimation of the Largest Lyapunov Exponent for increasing injection current using Wolf's algorithm. 


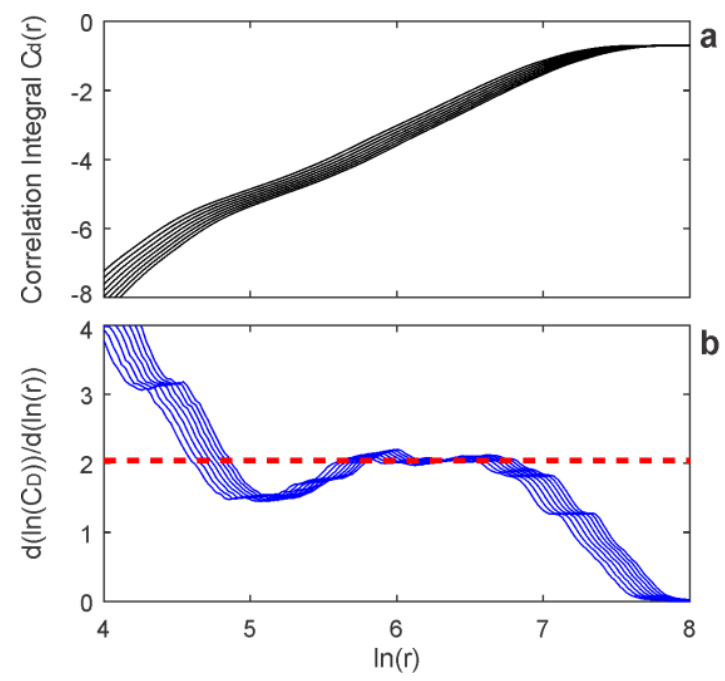

Figure 5.13. Results of the GP algorithm. In both panels, we show the evolution of the curves for increasing segment length $D$. Panel a) shows the evolution of the correlation integral $C_{D}$ - i.e. the average number of neighbouring points in the sphere of radius $r$. In panel $b$ ) we show the derivative $d\left(\ln \left(C_{D}\right)\right) / d(\ln (r))$ that leads to a converging correlation dimension for increasing length of segment $D$. Such convergence is clearly obtained around $\ln (r)=6.3$ where the curves reach a plateau around 2.04 .

Despite the value of the $K_{2}$-entropy is three orders of magnitude smaller than the one previously reported, this is due to the different time-scales of the dynamics as well as slower dynamics. For example, in [116] the average dwell-time is of the order of the nanosecond while here the microsecond scale is reached, which means that a different sampling rate was used in each case. The delay constant $(\tau)$ used for the $K_{2}$-entropy calculation is thus three orders of magnitude larger [116] leading to a significantly smaller value for the slower dynamics.

Therefore, all the observations and experiments carried out here confirm that we successfully generated polarization chaos in a solitary QW-VCSEL. We unambiguously identified chaos in the light polarization output of QW-VCSELs and confirmed it. In addition, we also confirmed that the reported dynamics is identical to the chaotic behaviour presented in [116]. Besides an excellent agreement with experimental observations, we further confirmed the chaotic nature of the dynamics via numerical processing techniques of experimental time-series. 


\subsection{Polarization Chaos Peculiarities}

In this subsection, we describe the main peculiarities and differences in the observed chaotic dynamics in QW-VCSELs when compared to previous observations made in QD-VCSELs. Even though the observations of the dynamics in QW-VCSELs described in the previous section show an excellent agreement with reported QDVCSEL behaviour, we also identify a few essential discrepancies. First, a second order transverse mode appears in the QW-VCSELs whereas only the fundamental mode was emitting in QD-VCSELs. We therefore carry out further investigations and analyse separately the different spatial modes of the strained QW-VCSEL to fully confirm that the chaotic dynamics is indeed of same nature. In particular, we must rule out random-like hopping between the fundamental and the second-order modes as the source of the mode-hopping dynamics. Second, we highlight the differences observed in terms of polarization orientation. In both cases, polarization chaos takes the shape of a random-like hopping between two elliptically polarized states, yet these states appear to have significantly different orientation and ellipticity. In the end, these discrepancies unveil the need for further theoretical studies to fully understand the origin and underlying mechanism behind them.

\subsubsection{Light-Intensity Curves with an Iris Diaphragm}

The dynamical measurements confirmed that we have triggered chaotic polarization fluctuations from QW-VCSELs. However, the observed dynamics differs from that of QD-VCSELs in some respects. For example, only the fundamental mode itself appears and is responsible for the chaotic dynamics in QD-VCSELs [116], i.e., the laser does not emit from any transverse mode. In contrast, higher-order transverse modes also affect the polarization dynamics in QW-VCSELs as discussed next.

The polarization resolved LI-curves for increasing injection current is plotted in Figure 5.14. For this setup scenario, the stress level \#8, from our stress scale, is used. Accordingly, notice that the laser starts emitting LP light along with the $90^{\circ}$ polarization direction. Then, a PS event happens at around $1.3 \mathrm{~mA}$ and the measured output power for polarization at $90^{\circ}$ is extinct. However, the output power for polarization at $0^{\circ}$ changes abruptly to $0.25 \mathrm{~mW}$, characterizing the first PS event as type I according to the literature. Finally, a second PS appears establishing the double PS event for an injection current above $6.2 \mathrm{~mA}$ accompanied by polarization hopping dynamics. The biasing threshold current is $0.50 \mathrm{~mA}$ and the second switching appears at a higher level of injection current (>6 mA), i.e., more than 12 times the threshold current. The complex polarization behaviour might be due to birefringence and anisotropy variation in the laser cavity caused by the in-plane strain. 


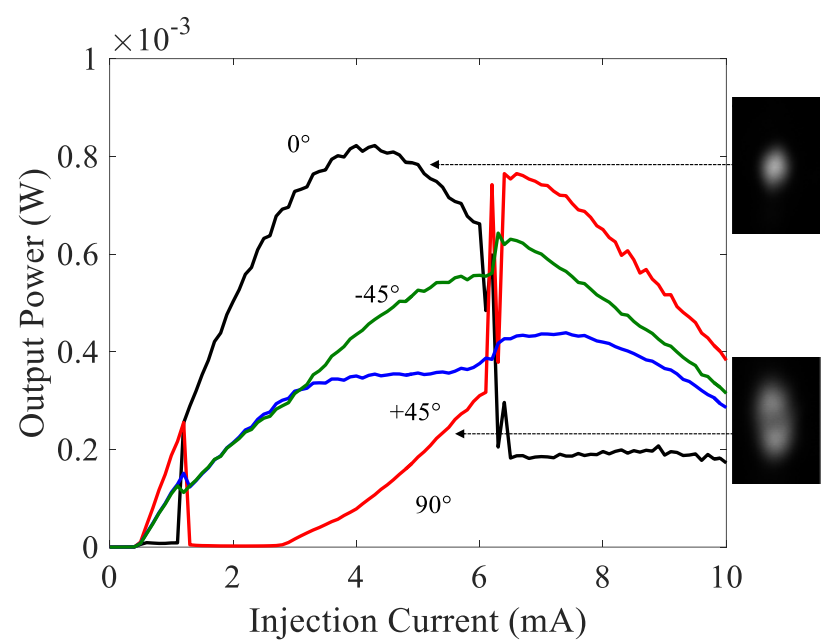

Figure 5.14. Polarization resolved output power for increasing injection current and for projection at $0^{\circ}, 90^{\circ}, 45^{\circ}$ and $-45^{\circ}$ with respect to the LP at threshold. A relatively high level of strain is applied onto the QW-VCSEL device and a double PS event along with polarization chaos is observed. The two insets show the spatial beam profile for polarization at $0^{\circ}$ and $90^{\circ}$ and for 6 $\mathrm{mA}$, confirming the existence of a $2^{\text {nd }}$ order mode at high current levels (bottom right inset).

Remarkably, a second higher-order transverse mode also contributes to the laser emission dynamics, which increases the system complexity, as shown in the inset of Figure 5.14, where the spatial beam profiles are shown for polarization projection at $0^{\circ}$ and $90^{\circ}$ (and considering $6 \mathrm{~mA}$ injection current), respectively. The scale of the transverse intensity profile of the beam is given in arbitrary unit (a.u.). It is clear the presence of the second higher-order mode for polarization at $90^{\circ}$. The second-order mode is expected to appear with a polarization orthogonal to that of the fundamental mode [144]. Hence, increasing the power above $3 \mathrm{~mA}$ for polarization at $90^{\circ}$ cannot be directly interpreted as a transition to EP eigenmode, which is typically reported before the onset of polarization chaos [116]. The manufacturer recommends a maximum injection current of $3 \mathrm{~mA}$, i.e. about 6 times the laser threshold. This recommendation aims not only at extending the device lifetime, but also at preventing the onset of higher-order spatial modes. Indeed, above this limit the laser will also emit at the second-order transverse mode. However, in order to confirm that the observed chaotic dynamics is mostly due to the fundamental mode, we constrain the influence of the second higher-order mode on the VCSEL dynamics. To do so, we consider an expansion of the QW-VCSEL output beam and add an iris diaphragm nearly fully closed before the fiber front-facet in the experimental setup so that it possible to spatially filter out the second higher-order transverse mode and then investigate the fundamental mode only. In this way, the polarization resolved LI curves for increasing injection current as well as the polarization projection at $0^{\circ}, 90^{\circ}$, $45^{\circ}$ and $-45^{\circ}$ considering an almost fully closed iris diaphragm is shown in Figure 
5.15. The two insets of this figure show the spatial beam profile for polarization projection at $0^{\circ}$ and $90^{\circ}$ for $6 \mathrm{~mA}$, respectively. Notably, the second higher-order transverse mode is completely suppressed, which allows for measurements of the fundamental mode only. In fact, the general dynamics has a behaviour similar to the previous case without an iris. More specifically, a double PS event is observed also when the second higher-order mode is filtered out from the QW-VCSEL output beam. Despite minor differences observed when comparing both results (Figure 5.14 and Figure 5.15), which are due to the high sensitivity of the experimental setup when the iris is included and the output beam is extended in terms of space range, we have enough evidences to conclude that the dynamics are mainly due to the fundamental mode. To further show that the influence of the second higher-order mode on the measurements of the output power beam can be constrained, which consequently allows for measurements and evaluation of the fundamental mode, we explore the optical spectra evolution with and without an iris diaphragm. Then, the polarization resolved optical spectra for polarization projection at $0^{\circ}$ and for injection current at 6 $\mathrm{mA}$, with and without an iris diaphragm, are shown in Figure 5.16. The power level difference between the fundamental mode and the second higher-order transverse mode is $6 \mathrm{~dB}$ without an iris (see Figure 5.16a). When the iris diaphragm is used, the output power difference between the modes becomes roughly $15 \mathrm{~dB}$, which is twice as large the previous case, as seen in Figure 5.16b. For lower values of injection current the difference is around $20 \mathrm{~dB}$. Finally, the wavelength spacing between both modes is around $0.8 \mathrm{~nm}$. Hence, the fundamental mode can be considered dominant for the measurements carried out.

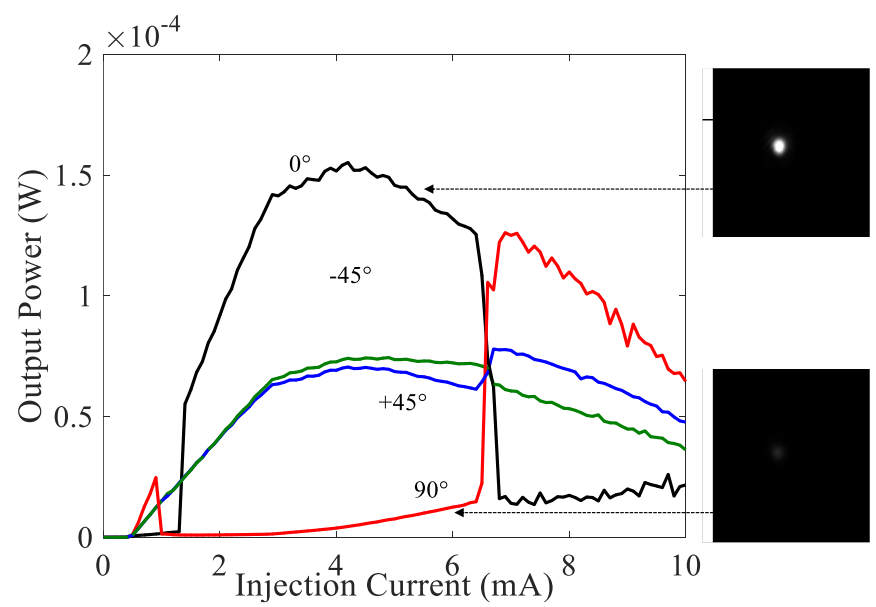

Figure 5.15. Polarization resolved light intensity curves for increasing injection current and for polarization projection at $0^{\circ}, 90^{\circ},+45^{\circ}$ and $-45^{\circ}$ with respect to the LP at threshold. An iris diaphragm almost fully closed was used to spatially filter out the second higher-order transverse mode. The scale of the transverse intensity profile of the beam is given in a.u.. It is observed a double PS event along with polarization chaos measuring the fundamental mode only. The two insets show the spatial beam profile for polarization at $0^{\circ}$ and $90^{\circ}$ for $6 \mathrm{~mA}$, confirming the predominance of the fundamental mode. 


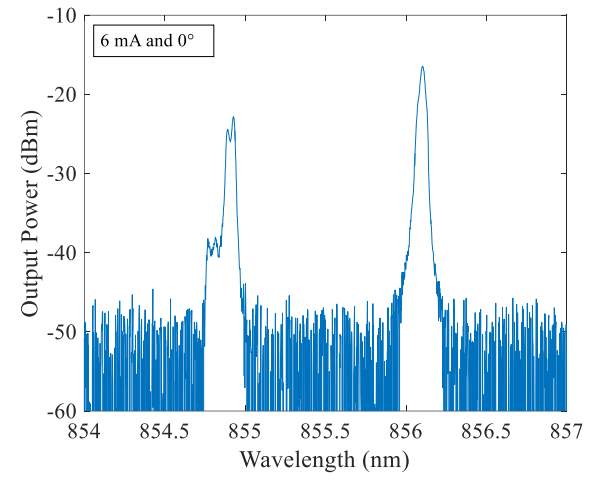

(a)

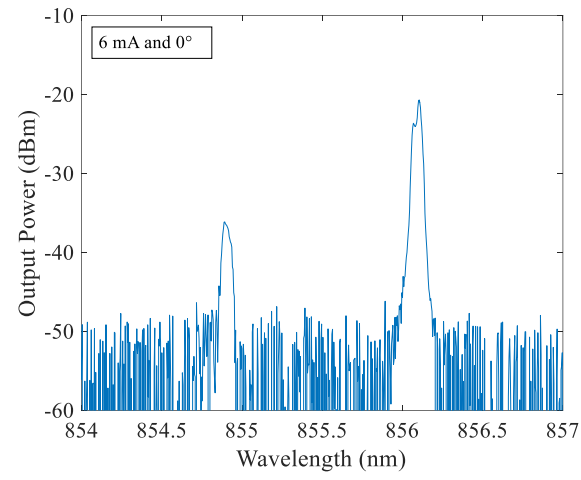

(b)

Figure 5.16. Polarization resolved optical spectra for projection at $0^{\circ}$ and for injection current at $6 \mathrm{~mA}$. a) Without the iris diaphragm. b) With the iris diaphragm included in the setup.

Likewise, the polarization resolved optical spectra for polarization projection at $90^{\circ}$ and for injection current at $6 \mathrm{~mA}$, without and with an iris diaphragm, is shown in Figure 5.17. The output power for the second higher-order mode is higher than for the fundamental mode for the case without an iris, see Figure 5.17a. However, with the iris diaphragm, both modes have basically the same output power, around - 39 $\mathrm{dBm}$ and a wavelength spacing difference around $1.2 \mathrm{~nm}$, see Figure $5.17 \mathrm{~b}$. Therefore, one can conclude that the power difference between the fundamental and second higher-order mode is significantly reduced when an iris diaphragm is used.

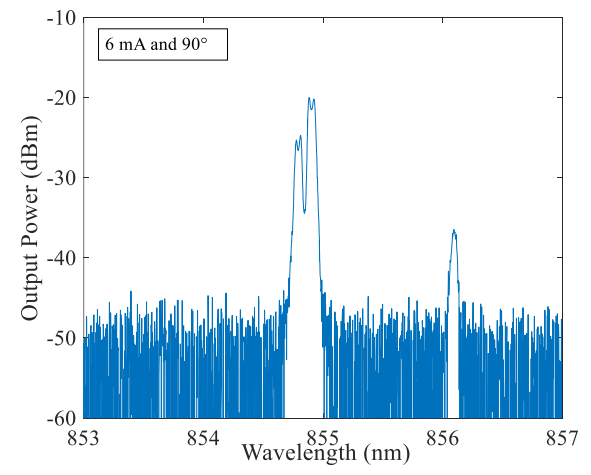

(a)

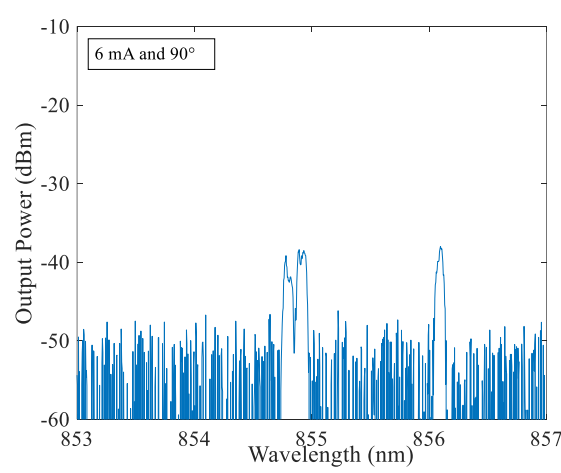

(b)

Figure 5.17. Polarization resolved optical spectra for projection at $90^{\circ}$ and injection current at 6 mA. a) Without the iris diaphragm. b) With the iris diaphragm included in the setup. 


\subsubsection{Polarization and Frequency-Resolved Optical Spectra}

The dynamical measurements addressed in the previous subsections are worth comparing with previous observations reported in [116]. The second-order mode is expected to appear with a polarization orthogonal to that of the fundamental mode [144]. Hence for increasing power, above $3 \mathrm{~mA}$, and for polarization at $90^{\circ}$, it cannot be directly interpreted as the transition through EP eigenmode, typically reported before the onset of polarization chaos [116]. We therefore need to make sure the observed random-like hopping between polarization states is not a switching between spatial modes. To this end, we exploit the wavelength difference between the two spatial modes ( $>1 \mathrm{~nm}$, see Figure 5.18a and Figure 5.18b to show the frequency-resolved light-intensity (LI) curves for polarization at $0^{\circ}$ and $90^{\circ}$ ). Thus, we record the optical spectra at different current levels, and estimate the output power for each spatial mode by integrating the spectral contribution of the corresponding peak.

We set a threshold at $-42 \mathrm{dBm}$ below which we consider that there is only noise contribution and no signal. These LI curves are shown in Figure 5.18c and Figure $5.18 \mathrm{~d}$ for polarization at $0^{\circ}$ and $90^{\circ}$, respectively. We observe that for both polarization orientations the contribution of the second order mode remains roughly constant while a large and sudden change is observed in the polarization of the fundamental mode. Hence, we confirm that the second switching is mainly due to the fundamental mode itself.
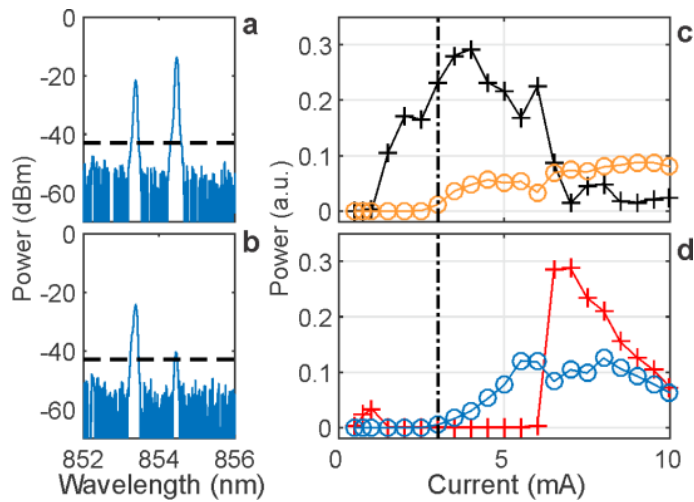

Figure 5.18. Frequency resolved LI-curves. $(a, b)$ typical two-mode optical spectra recorded at 4 $\mathrm{mA}$ for polarization at $0^{\circ} \mathrm{a}$ ), and $90^{\circ} \mathrm{b}$ ). The horizontal dashed lines indicate the threshold above which spectral data are integrated to estimate the power of each mode. (c, d) Frequency resolved LI curves for polarization at $0^{\circ} \mathrm{c}$ ), and $90^{\circ} \mathrm{d}$ ): the black (red) solid line with crosses represents the fundamental mode while the orange (blue) solid line with circles represents the second order mode. The vertical dash-dotted line indicates the threshold for the second order mode, i.e. about $3 \mathrm{~mA}$. 
Finally, when zooming-in around the switching point, we also observe that the fundamental mode undergoes a short transition through a slightly elliptical polarization just before the second switching point, around $6.2 \mathrm{~mA}$ that can be confirmed by the inset panel of Figure 5.19. This is important as this transition has been shown to be an essential step in the route to polarization chaos [116]. We obtained equivalent results when spatially filtering the second order mode with an iris diaphragm after expansion of the laser beam.

Next, we provide additional details on the emergence of the higher-order mode and its polarization. Then, we show the frequency resolved LI curves for polarization projections at $0^{\circ}, 90^{\circ}, 45^{\circ}$ and $-45^{\circ}$ in Figure 5.19. As already mentioned, the second switching that appears around $6.2 \mathrm{~mA}$ is clearly a switching of the fundamental mode since a large exchange between polarization at $0^{\circ}$ and $90^{\circ}$ for the fundamental mode can be realized, whereas for the second order mode only very minor changes are noticed. In addition, we observe that the fundamental mode is almost identical for projections at $45^{\circ}$ and $-45^{\circ}$, which confirms that it is nearly linearly polarized until the region of the second switching, i.e. below $6.2 \mathrm{~mA}$.

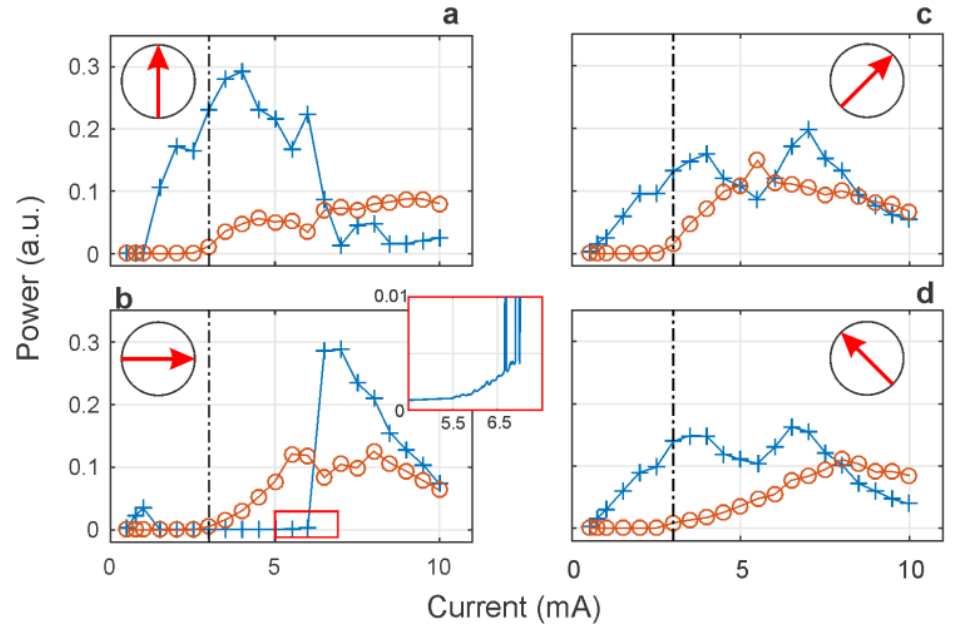

Figure 5.19. Frequency resolved LI-curves for polarization projection at a) $0^{\circ}$, b) $90^{\circ}$, c) $45^{\circ}$, and d) $-45^{\circ}$. For each panel the projection orientation is also indicated by the red arrow in the black circle. In all panels, the blue line with crosses shows the evolution of the fundamental mode while the orange line with circles represents the second order mode. The inset in panel (b) shows a zoom around the switching point delimited by the red rectangle. 


\subsubsection{Orientation of the Polarization Modes}

This subsection addresses the dynamical differences observed between QW-VCSELs and previously reported QD-VCSELs. We observe chaotic dynamics that resembles a random-like hopping between two EP states. The fast oscillations that correspond to the rotation around the scrolls of the chaotic attractor have been filtered out [142], and are accounted here as the oscillations around $8 \mathrm{GHz}$ shown by the RF spectra measurements.

In QD-VCSELs, the two EP states have a strong ellipticity and are typically symmetrical with respect to the polarization direction of the laser eigenmodes, as schematically illustrated in Figure 5.20b. Small asymmetries between EP state 1 (EP1) and EP state 2 (EP2) have already been reported, but were deduced either from asymmetries in the residence time statistics [140] or from the appearance of dynamical bistability [137], since no significant asymmetries could be realized between the polarization orientation of the two EP states. Consequently, the largest amplitude for the random-like hopping is always obtained for projection at $45^{\circ}$ of the polarization direction at threshold [138]. In contrast, QW-VCSELs subjected to stress have a distinct behaviour as illustrated in Figure 5.20c.
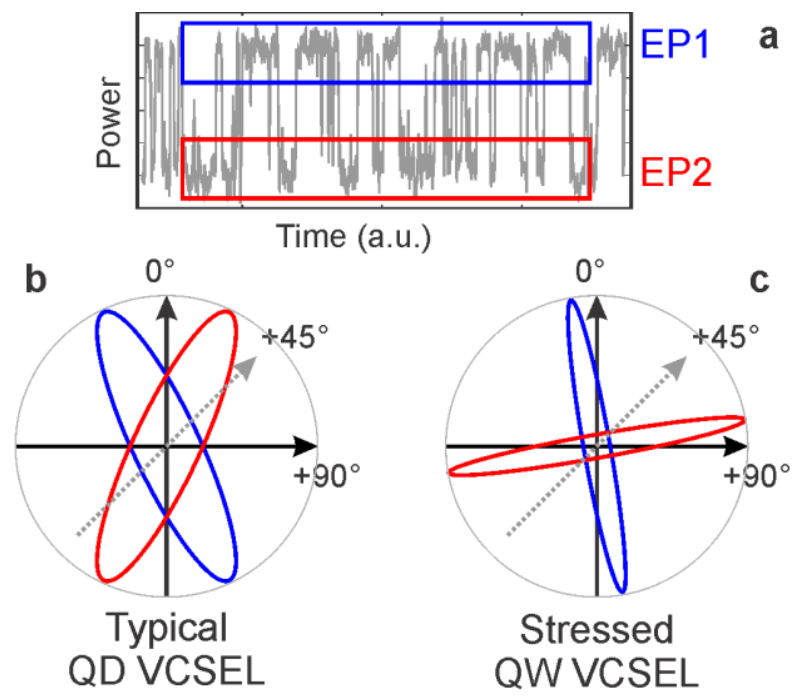

Figure 5.20. Schematic orientation view of the elliptically polarized states. a) Typical polarization chaos time-series showing a random-like hopping between two elliptically polarized states EP1 and EP2 highlighted in blue and red, respectively. The orientation of these two states is shown with respect to the laser eigenmodes for a typical case in QD VCSEL and for the stressed QW VCSEL described here in b) and c), respectively. 
The two EP states are very close to the laser eigenmodes themselves and hence show no sign of symmetry with respect to their direction of polarization. In this way, the two EP states are symmetrical to the polarization direction around $45^{\circ}$. Consequently, the random-like hopping is observed for projections at $0^{\circ}$ and $90^{\circ}$, but not at $45^{\circ}$ as previously reported [137], [140]. Moreover, the ellipticity of the two states appears to be much smaller than that of the EP states in QD-VCSELs. Indeed, one can clearly observe that the ellipticity (thickness) of the EP states in QW-VCSELs is smaller than in QD-VCSELs. This is due to the extinction ratio obtained from the measured output power between the main polarization direction and its orthogonal direction. For example, the extinction ratio measured in QW-VCSELs is high, which leads to small ellipticity. In contrast, the extinction ratio obtained in QD-VCSELs is low, hence resulting in large ellipticity. Finally, the asymmetries in the residence time statistics (about 2 orders of magnitude difference) and the frequency difference between the periodic solutions oscillating around the two EP states (of about $600 \mathrm{MHz}$ ) are of the same order of magnitude as those reported in [137], [140]. Therefore, the differences might be limited only to the EP state orientation and ellipticity, with minor impact on the other properties of the dynamics.

\subsection{Summary and Perspectives}

As described in this chapter, we were able to trigger chaotic dynamics from commercial QW-VCSELs using a mechanical strain, where deterministic chaos is generated without any additional complexity of optical feedback, parameter modulation or optical injection. The absence of time-delay such as in schemes based on optical feedback entirely removes the need for arduous time-delay concealment. We experimentally demonstrated that polarization chaotic dynamics can be obtained in a consistent and easily reproducible way from ordinary commercial semiconductor laser diodes (QW-VCSELs) in a free-running configuration using a simple, low-cost, easily replicable holder to introduce anisotropic strain in the laser cavity.

Although the emergence of a higher-order mode has rendered the system more complex, all measurements and analyses successfully confirmed that we were able to generate deterministic polarization chaos from a solitary QW-VCSEL. After the confirmation of a higher-order mode for higher injection currents, we investigated the influence of the second higher-order transverse mode on the VCSEL dynamics via an expansion of the output light beam by including an almost fully closed iris diaphragm in the experimental setup to spatially filter this mode out. Then, we obtained similar polarization dynamics as in previous experiments, which helped us to conclude that the observed dynamics are due to the fundamental mode only. We carried out further measurements so that the nature of the dynamics could be 
unequivocally confirmed. In this way, we investigated the differences in wavelength and optical power between the fundamental mode and second order transverse mode, also without and with an iris diaphragm. As expected, the differences between them were considerably large. This showed, once again, that the polarization dynamics mainly appears due to the fundamental mode. Besides an excellent agreement with previous experimental observations in QD-VCSELs, we also unambiguously confirmed the chaotic nature of the dynamics via numerical processing of experimental time-series data. From a fundamental point of view, this result also confirms that the chaotic dynamics originated from the VCSEL structure itself and is not related to the quantum dots as gain medium, which means that all types of VCSELs can be potentially turned into chaos generators.

However, we pointed out a few differences in the observed chaotic dynamics between QW-VCSELs and those previously reported in the literature for QDVCSELs, which are: hopping event happening for polarization projection at $0^{\circ}$ and $90^{\circ}$ for QW-VCSELs, whereas it happens only for polarization projection at $45^{\circ}$ for QD-VCSELs. Two spatial modes are observed in QW-VCSELs, whereas only the fundamental mode is observed in QD-VCSELs. Finally, the EP states are not symmetric with respect to their direction of polarization in QW-VCSELs, whereas as reported to QD-VCSELs, these two EP states have a strong ellipticity and are symmetric with respect to the polarization direction of the laser eigenmodes.

In summary, the chaotic dynamics observed from off-the-shelf QW-VCSELs subjected to mechanical strains exhibits the following features:

1- Double PS event (type I switching followed by type II);

We destabilized the laser dynamics and successfully obtained a double PS event among with chaotic fluctuations by gradually increasing the amount of applied stress onto a QW-VCSEL.

2- An abrupt frequency shift and a second PS event appearing simultaneously; We investigated the radio-frequency spectra evolution for the output beam of the VCSEL subjected to mechanical strain, where frequency jumps were observed near the injection current region of the double PS event. More specifically, a sudden frequency change was observed near the second switching that clearly resembles the limit cycle bistability, previously reported in chaotic QD-VCSELs [47]. Moreover, we observed the emergence and contribution to the polarization dynamics also in the low frequency range bellow $1 \mathrm{GHz}$. The lack of any featured frequencies implies that the dynamics does not exhibit any characteristic frequency, which also suggests a chaotic behaviour. 
3- The dynamics appears as a random-like hopping between two polarization eigenmodes and an average residence time that decreases exponentially as the injection current increases;

We carried out dynamical measurements concerning the time-series traces of the polarization dynamics and we clearly observed random-like hopping dynamics between two polarization states similar to those previously observed in QD-VCSELs. We further noticed that the residence time largely reduces as the injection current increases and that the polarization eigenmode hopping is asymmetric, with the laser changing frequently between two EP eigenmodes, but staying on average longer in one level.

4- A positive largest Lyapunov exponent obtained via the Wolf's algorithm; Using Wolf's algorithm and the experimental time series data, we confirmed that the dynamics exhibit a non-zero but finite largest Lyapunov exponent coherent with the chaotic interpretation of the dynamics.

5- The GP algorithm converges to a non-zero value of the $K_{2}$-entropy $\left(\approx 5.210^{-3}\right.$ $\mathrm{ns}^{-1}$ ), and a corresponding correlation dimension equals $D_{2} \approx 2.04$;

We used the GP algorithm that gives an estimate of the correlation dimension $D_{2}$ as well as the Kolmogorov entropy $\left(K_{2}\right)$, where $K_{2}$ is zero for periodic or quasiperiodic systems, positive for chaos and $K_{2}=\infty$ for purely stochastic processes. Deterministic chaos was identified with the values provided by the GP algorithm.

6- Frequency resolved measurements confirm that the results are mostly due to the evolution of the fundamental mode despite the emergence of a second higher-order transverse mode;

We observed a short transition through elliptically polarized states using frequency resolved measurements.

Therefore, the practical impact and perspectives of the work described in this chapter are in short, four-fold:

i) To follow the recent progress of alternative strain-inducing techniques that lead to self-pulsations of tens of $\mathrm{GHz}$ and beyond, and to employ the proposed chaos generator in combination with other techniques. This might have the potential to achieve chaotic dynamics at mm-wave frequencies, which certainly represents a major paradigm-shift in the design of chaotic devices and opens new possibilities of applications; 


\section{POLARIZATION CHAOS FROM FREE-RUNNING QW-VCSELS}

ii) To motivate further development of solutions based on QW-VCSELs capable of generating chaotic signals without any mechanical forcing. This might eventually open innovative ways for the realization of emerging applications with high-integrability and -scalability, where hundreds of individual optical chaotic sources are required to increase requirements for applications like random bit generation, cryptography or large-scale quantum networks;

iii) To explore the proposed chaos generator towards solutions for highly-secure and robust optical networks for transmission of data information via synchronization of chaotic QW-VCSELs. This would not only help combat security vulnerabilities at the physical layer, but also foster the convergence with access networks technologies;

iv) To explore deterministic chaos in combination with OCDMA techniques in the way that a chaotic signal can be modulated with data information and encoded before transmission through a network. This potentially might not only achieve higher levels of security against eventual eavesdroppers, but also might increase the capacity of networks in terms of simultaneous users. 


\section{Chapter 6 Conclusions and Perspectives}

In this chapter, the original contributions presented in this thesis are summarized and the main achievements are briefly described highlighting their impact on the field. Future perspectives are also discussed, showing that this research has opened new possibilities to be explored.

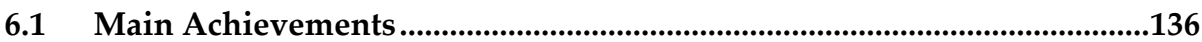

6.2 Future Perspectives ............................................................................................140

6.3 Summary of the Original Contributions .........................................................144 


\subsection{Main Achievements}

We have advanced the current state-of-the-art of two key subjects in this doctoral research, namely, the analytical performance investigation of flexible access networks based on OCDMA techniques capable of providing multiservice multirate transmissions, and the experimental proposal of an innovative optical chaos generator setup based on cost-effective commercial QW-VCSELs for secure communications purposes. These two key proposals entailed additional contributions and achievements that can be further subdivided into six major parts, namely, new analytical formalisms for evaluation of flexible OCDMA networks (Chapter 3), performance evaluation of networks under different traffic conditions (Chapter 4), performance evaluation of IP traffic transmissions using 1-D or 2-D codes under different network architectures (Chapter 4), performance evaluation of networks using the multicode technique (Chapter 4), packet delay performance evaluation for flexible networks (Chapter 4), and a cost-effective source of optical chaos generation for secure communications purposes (Chapter 5). Our main research contributions and results obtained for each of these six parts are summarized in what follows.

\section{1) New analytical formalisms for performance evaluation of flexible OCDMA networks in terms of BER, packet throughput and delay (Chapter 3).}

We proposed for the first time a new mathematical expression for evaluating the BER performance of flexible OCDMA networks based on the binomial distribution for the MAI. The BER expression can be used to evaluate the network performance considering a general number of users' classes present in the system. We also proposed for the first time a robust analytical formalism for evaluating the packet throughput and packet delay of flexible OCDMA networks considering Poisson, binomial and Markov chain approaches for the distribution of composite packet arrivals. The Markov chain approach was defined as benchmark against which the throughput performance can be compared. Remarkably, the analytical formalisms proposed here account for a general number of distinct user classes as well as general probability of interference among users with good correlation properties. In this manner, the developed formalisms can be successfully used to carry out performance analysis of flexible OCDMA networks under any number of user classes as well as for most 1-D and 2-D codes with good correlation properties. The packet throughput and delay expressions can be applied for performance evaluation of advanced modulation formats such as PSK if a new BER expression is provided. In addition, the packet throughput and delay expressions can be further used to assess the performance of flexible networks with either MAI as the only degradation source or with MAI plus other deleterious sources such as time jitter, beat noise and channel 
impairments in general, again as long as a new BER equation is provided. Finally, we defined the convergence of conventional single-rate networks towards flexible multirate networks as a validation criterion and used it to validate and to assess the robustness of the new developed formalisms.

\section{2 ) Performance evaluation of flexible OCDMA networks assuming different scenarios and traffic conditions (Chapter 4);}

Here, we addressed and compared for the first time the performance evaluation of a FFH-OCDMA network capable of providing multirate transmissions using the binomial and gaussian distributions for the BER. We showed via numerical simulations that the gaussian-based BER underestimates the network performance in unacceptable levels while the binomial-based BER results are accurate. Accordingly, we showed that gaussian-based BER is not a good approximation to assess the performance of flexible networks.

We further addressed for the first time the packet throughput and delay performance of flexible MWML-OOC OCDMA networks with users assigned with either 1-D or 2-D codes assuming binomial, Poisson and Markov chain approaches for the composite packet arrivals with the latter defined as benchmark. We proved via numerical simulations that the Poisson-based throughput equation is a poor approximation as it incorrectly predicts the number of successfully transmitted packets for most values of offered load, especially for user' classes defined with high code weight. Even under favorable conditions such as for a large number of simultaneous users in the system, the Poisson approach kept providing inaccurate results when compared to Markov results (benchmark). The binomial-based throughput results, by its turn, agreed really well with those obtained with the benchmark. Indeed, the binomial-based results showed a perfect overlap for all network scenarios investigated. Moreover, it proved to be numerically more efficient and computationally faster than the Markov chain approach. The Markov approach is constrained by time and computationally expensive with decreasing numerical performance as the number of users is increased. Hence, we concluded that the new introduced formalism based on the binomial approach has an excellent trade-off between computational effort and accuracy.

\section{3 ) Performance evaluation of IP traffic transmissions over OCDMA networks assuming 1-D or 2-D users' codes (Chapter 4);}

Here, we proposed for the first time a new OCDMA network architecture supporting multirate transmissions of IP data traffic. The new network architecture is independent of the spreading code employed and does not require any new optical processing technology. We introduced two distinct network 
architectures that use incoherent receptors based on intensity modulation directdetection scheme and provide asynchronous transmissions.

The first network architecture employs 1-D encoding schemes and ODLs, where MWML-OOC are assigned to users. The channel utilization of the proposed network is equally varied for all classes at the same instance to maintain a fair transmission rate. Simulation results showed that the proposed network performs better when its users are defined with high code weight or when the channel utilization is low. The second network architecture employs 2-D encoding schemes and MBG-based encoders and decoders designed to a specific transmission rate or tuned to a higher rate according to a user demand. In this network, FFH-based codes are assigned to users and the encoders adjusting time is low since they are adjusted for several packets belonging to the same user rather than being tuned for every incoming packet. Moreover, the buffer delay is significantly reduced when compared to traditional routing schemes since each packet is buffered only twice at the edges of the network. We address the performance of the proposed network under different channel utilization conditions, where the utilization is equally varied for all classes at the same instance to fairly maintain the original designed transmission rate. Simulation results showed that the performance of users' classes assigned with low-code weight is more susceptible to channel utilization variations than those of a user class with high code weight. The proposed flexible OCDMA architecture can be regarded as a prospective candidate for future access networks with the capacity of accommodating several classes of users under different requirements of services and transmission rates.

\section{4 ) Performance assessment of OCDMA networks capable of providing multirate transmissions via multicode technique and comparison with classical multilength code technique (Chapter 4);}

Here, we address for the first time the performance of a 2-D MWML-OOC OCDMA network providing multirate transmissions via the assignment of two codes for each single user (multicode technique). We derived new packet throughput expressions considering binomial distribution for the MAI and Poisson distribution for the composite packet arrivals. We compared the network performance against the classical multilength code technique assuming the same network operating conditions. Interestingly, the multicode technique outperformed the multilength one and showed that it can successfully deliver more packets. In fact, the multicode technique supports lower levels of MAI for the desired user which allows for an increase in the number of of packets delivered with success. In contrast, from a practical perspective, the multilength technique only requires a single encoder and decoder pair whereas the multicode technique requires several pairs according to the desired transmission rate. To 
the best of our knowledge, this is the first report on the packet throughput performance of flexible multirate OCDMA networks.

\section{5 ) Performance evaluation of flexible OCDMA networks in terms of packet delay (Chapter 4);}

The packet delay is assessed assuming Poisson, binomial and Markov chain approaches for the composite packet arrivals with the latter defined as benchmark. The performance of a two-class flexible OCDMA network employing the so called 2-D multiweight multilength optical orthogonal code (MWML-OOC) is addressed. Numerical results showed that the packet delay of flexible OCDMA networks can be underestimated in unacceptable levels when carried out using the Poisson approach. In contrast, the binomial approach proved to be just as accurate as the Markov chain approach, in addition to being an elegant solution by its simplicity and low computational cost. To the best of our knowledge, this is the first report on the packet delay performance of flexible multiservice multirate OCDMA networks.

\section{6 ) Generation of optical chaos from commercial QW-VCSELs (Chapter 5).}

Here, it is demonstrated the pioneering work of chaos generation from an offthe-shelf truly cost-effective QW-VCSEL operating in a free-running configuration. Briefly, deterministic chaos is triggered from a commercial QWVCSEL subjected to mechanical stress via a simple and custom-made holder that renders the system easily reproducible. Deterministic chaos was then generated without any additional complexity such as optical feedback, parameter modulation or optical injection. Even though the onset of a higher-order mode has led to a somewhat larger complexity, it was fully confirmed that the observed dynamics are indeed deterministic chaos with a behavior similar to previous observations in QD-VCSELs. For example, besides an excellent agreement with experimental observations, it was also clearly confirmed via numerical processing techniques of the experimental time-series that the reported complex dynamics are of chaotic nature.

This work has solved for the first time the practical problem of unavailability of devices capable of generating polarization chaos and has shown that potentially all VCSELs might be capable of generating chaos. This optical chaos generator might significantly influence emerging applications that are currently impractical or infeasible due to complexity, prohibitive cost, and limited availability of known solutions. This work has therefore paved the way for future research on polarization chaos, its properties and related applications that can largely benefit from the simplicity, compactness and robustness of the proposed approach. 
It also motivates further research on the development of strained VCSELs capable of generating chaotic polarization fluctuations without any external mechanical forcing. This opens new possibilities not only for advanced chaotic laser systems and applications, such as two-dimensional arrays of chaotic devices comprising hundreds of individual sources to increase requirements for applications like random bit generation, cryptography or quantum networks, but also for new higher security platforms in optical access networks.

\subsection{Future Perspectives}

On one hand, our contributions in optical networks pave the way towards flexible (dynamical bandwidth allocation) OCDMA networks, which are potential candidates for next generation passive optical networks, where support to broadband services and to diversified patterns of data traffic as well as conscious allocation of the available bandwidth, will be crucial network requirements. On the other hand, our contributions in cost-effective optical chaos generation pave the way towards not only the wide spread use of commercial QW-VCSELs for chaos-based applications, but also to innovative chaotic applications as well as improvements of current solutions, in addition to solving for the first time the practical problem of devices unavailability for chaos generation. Based on the work described in this doctoral thesis along with the main achievements summarized above, we can draw some interesting perspectives for future work. Briefly, separated in two main topics, they are:

\section{i) Flexible OCDMA networks}

\section{To address the performance of hybrid optical networks;}

To investigate the packet throughput performance of hybrid networks based on TDM/OCDMA or WDM/OCDMA technology. This is appealing because technology candidates for access networks are expected to support smooth migration from current infrastructure to future network architectures. For example, hybrid TDM/OCDMA networks can be considered a smooth partial upgrade before an eventual total migration towards OCDMA technology. OCDMA technology might be cogitated as a prominent candidate for the future upgrade definitions of access networks, since a demand for a revolutionary PON architecture is expected for the coming decade without the requirements of full backward compatibility with previous technologies. 
2. To address the performance of optical access networks considering other sources of noise inherent to the optical channel;

To extend even further the proposed BER and packet throughput analytical formalisms considering the inclusion of deleterious noise sources such as beat, thermal, shot and relative intensity noises, in addition to nonlinear effects from an optical channel and jitter effect. Even though digital signal processing might compensate inherent fiber impartments (such as chromatic and polarization mode dispersion), it is still worth to theoretically investigate the network performance as it provides a more complete, and therefore more realistic view of the system.

\section{To propose multirate networks based on advanced modulation formats;}

To propose new network architectures capable of providing multiservice multirate transmissions based on advanced modulation formats. Also, to explore techniques based on adaptive modulation formats so that different modulation formats can be used according to traffic demand. To extend the proposed BER and packet throughput analytical formalisms considering advanced modulation formats based on PSK. For example, it has recently become of general interest to assess the performance of flexible networks using BPSK or QPSK schemes since they are considered potential candidates to replace OOK schemes in access networks in the foreseeable future.

External modulation techniques with a laser producing continuous output beam are required along with a device such as Mach-Zehnder interferometer (MZI) to implement advanced modulation formats. The principle of a MZI is to divide the pulse amplitude and phase modulation of one part of the signal to produce constructive or destructive interferences after recombining it. MZI-based modulators can be used to implement modulation formats such as BPSK and QPSK to transmit one or several bits per symbol. For example, for one bit per symbol, BPSK transmits the optical field signal for both the " 1 " and " 0 " data bits by changing the 0 and $\pi$ phases, respectively. In contrast, two bits can be simultaneously transmitted with one phase modulated waveform via QPSK. Surely, such modulation formats require phase information to retrieve the transmitted data bits, where the demodulation process is normally based on balanced detection along with a local oscillator signal.

\section{To propose multirate networks based on coherent encoding techniques;}

To extend the proposed packet throughput analytical formalisms for OCDMA networks based on coherent detection and manipulation of the signal's phase to extract any phase information carried by a transmitted 
signal. Coherent networks have received a great deal of attention nowadays due to the maturity of optical device technology in dealing with phase coherence information. Even though coherent networks have been largely investigated in the literature, very little attention has been paid to flexible schemes with support to multiservice multirate transmissions. In addition, coherent networks can be classified into two different schemes based on the way the phase encoding is applied to the field of an optical signal, namely, spectral phase-encoded time-spreading (or spectral phase coding (SPC)), and time-spreading (or time phase coding (TPC)). In the SPC scheme, the encoding is performed directly in the frequency domain by means of phase shifts induced in the different spectral components that form the pulse. In the TPC scheme, by its turn, the encoding is performed in the temporal domain by manipulating the pulses' phase via encoding devices based on superstructured fiber Bragg gratings, planar lightwave circuit, or multiport encoders/decoders.

\section{To propose hybrid networks based on free-space optical technologies;}

To design new hybrid network architectures based on OCDMA and freespace optical (FSO) technologies benefiting from common features such as asynchronous and high-data rates transmissions so that high-speed communication services for last-mile access networks can be provided. Freespace optical networks have been recently considered due to their potentially large transmission capacity and license-free operation as opposed to license paid microwave systems. Atmospheric optical systems are appropriate candidates for extremely secure, high data rate, costeffective, wide bandwidth communications. A feature that has driven FSO technology to conquer its space in the current scenario is the use of the socalled Bessel light beam. Bessel beams feature propagation-invariant or diffraction-free, which means that they do not diffract and spread out as they propagate. Bessel beams are also self-healing, meaning that the beam can be partially obstructed at one point, but will re-form at a point further. This allows FSO systems to overcome obstructions during the propagation path such as atmospheric turbulences, and consequently avoid performance degradation of the network. In addition, there are many techniques available to overcome the adverse effects of atmospheric turbulence. For example, the use of adaptive optics to compensate the distortion of the wave-fronts and the use of cooperative protocols along with multiple relays employing detect-and-forward. The combination of both FSO and OCDMA technologies might be considered as a prospective solution to deliver multiservice and multirate transmissions, where fiber infrastructure deployment is impractical or deficient with no provision of broadband 
technologies such as in rural areas, remote islands and places with critical geographical difficulties. Finally, because of the scalability and flexibility of FSO technology, optical wireless solutions can be deployed in several enterprise applications including last-mile connectivity, private line replacement, mobile wireless backhaul, and disaster recovery, to name but a few.

6. To propose innovative designs of encoders and decoders exclusively for flexible networks;

To design and propose novel encoders and decoders with adaptive capabilities so that the employed code sequence can be dynamically changed according to the current data traffic demand of a certain user. The current lack of mature solutions concerning new adaptive encoding techniques for flexible networks limit the wide spread solutions based on OCDMA.

\section{ii) Deterministic chaos generation from commercial QW-VCSELs}

\section{Secure optical networks based on chaos synchronization;}

To use the proposed optical chaos generator for transmission of data information via synchronization of a pair of chaotic QW-VCSELs. This would eventually provide higher levels of secure communication between two entities at the physical layer. Moreover, to investigate not only synchronization, but also diverse types of synchronization schemes available in chaos communication.

\section{Chaos generation for mm-wave frequencies from strained QW-VCSELs;}

To follow the recent progress of alternative strain-inducing techniques that lead to self-pulsations of tens of $\mathrm{GHz}$ and beyond, and to employ the proposed chaos generator in combination with other techniques. This might have the potential of producing chaotic dynamics at mm-wave frequencies, which certainly represents a major paradigm-shift in the design of chaotic devices and opens new possibilities of applications.

\section{Optical chaos CDMA networks;}

To explore a chaotic carrier in combination with OCDMA techniques so that the same can be encoded before being sent to transmission over a network. This would not only provide highly-secure transmissions on such a communication channel, but also would potentially increase the capacity of 
a network in terms of simultaneous users. Even though there are plenty of works addressing the generation of chaotic sequences in CDMA networks, only a very few works have proposed a device generator of chaotic code sequences for OCDMA networks. Regardless of the network as well as in contrast to the classical idea of generation of chaotic sequences, it would be worth investigating a network with chaotic signals from QW-VCSELs applied to conventional pairs of encoders and decoders, such as the ones based on time-spreading or 2-D encoding schemes. This would eventually enable current OCDMA networks to become chaotic. An alternative to apply chaotic signals to an OCDMA encoding scheme would be to use bipolar codes and polarization shift keying modulation. For example, a light beam transmitting on two polarization states can be used to encode chips that indicate a bipolar code $\{-1,+1\}$.

\section{Chaos generation from commercial QW-VCSELs without any mechanical forcing;}

To motivate further developments of QW-VCSELS capable of generating chaotic signals without any mechanical forcing. This might eventually open innovative ways for the realization of emerging applications with highintegrability and -scalability, where hundreds of individual optical chaotic sources are required to support applications like random bit generation, cryptography or large-scale quantum networks.

\subsection{Summary of the Original Contributions}

The design of highly-secure, flexible bandwidth and high-capacity networks to provide transmissions of diverse and bursty data traffic has become a complex task to be accomplished nowadays. In addition, the capacity and resources of conventional optical fiber networks are not infinite as previously thought, and the ever-increasing data traffic demand is at an all-time high. Accordingly, we are convinced that the proposed analytical formalisms for assessing the performance of flexible OCDMA networks are useful tools for network designers to select the most effective architecture or the design constraints a system can support so that they satisfy expected requirements. In this way, the underlying physical network resources can be efficiently used, especially if one considers the bandwidth capacity of current conventional optical fibers is a finite resource and should be used more efficiently. Regarding network security, current networks' schemes normally rely on encryption methods to provide security data transmissions against potential eavesdroppers. These methods are implemented at higher protocol layers, which 
might be inefficient. In this context, we have tackled the security and vulnerability issues in optical networks by means of a novel and cost-effective chaotic VCSEL source which be a potential solution not only for OCDMA networks, but optical networks as a whole. Finally, the major contributions of this doctoral thesis are summarized and listed as follows:

- We proposed novel analytical formalisms for assessing the performance of flexible OCDMA networks. We derived new mathematical expressions for BER, packet throughput and delay performance evaluation;

- We proposed a new IP transmission architecture over OFFH-CDMA network capable of providing multirate transmissions for applications in flexible optical access networks. We derived new expressions for the decoder BER, total BER, PER and packet throughput to evaluate the network performance;

- We proposed a new IP transmission architecture over 1-D MWML-OCDMA networks capable of providing multiservice multirate transmissions for flexible optical access network applications. This architecture can be considered useful and robust from a practical point of view, since it does not require any new optical processing technology;

- We addressed the packet throughput performance evaluation of multiservice multirate OCDMA networks based on two distinct multirate techniques, namely, multilength code and multicode. To accomplish this, we derived new analytical formalisms considering both multirate techniques and compared their performance;

- We addressed the packet throughput and packet delay of flexible OCDMA networks based on three distinct mathematical approaches as well as on different network traffic conditions and on distinct 1-D and 2-D encoding schemes;

- We proposed a cost-effective optical chaos generator based on commercial QW-VCSELS operating in a free-running configuration for secure communications purposes. 



\section{List of Publications}

\section{Peer-reviewed Journal Articles}

1. T. R. Raddo, K. Panajotov, B.-H. V. Borges, and M. Virte, "Polarization chaos from a commercial free-running laser diode", Scientific Reports, Nature Group, 2017, submitted.

2. T. R. Raddo, A. L. Sanches, and B.-H. V. Borges, "Packet Delay Performance of Flexible Optical CDMA Networks", IEEE/OSA J. Opt. Commun. and Netw., 2017, to be submitted.

3. T. R. Raddo, A. L. Sanches, I. T. Monroy, and B.-H. V. Borges, “Throughput performance evaluation of multiservice, multirate OCDMA in flexible networks," IEEE Photonics Journal, vol. 8, no. 1, pp. 1-15, February 2016.

4. T. R. Raddo, A. L. Sanches, J. V. dos Reis Jr., and B. -H. V. Borges, "A new approach for evaluating the BER of a multirate, multiclass OFFH-CDMA system," IEEE Communications Letters, vol. 16, no. 2, February 2012.

\section{International Conferences Proceedings Articles}

1. T. R. Raddo, A. L. Sanches, I. T. Monroy, and B.-H. V. Borges, "Packet throughput performance of multiservice, multirate OCDMA in elastic networks", in Proc. IEEE International Conference on Communications, Kuala Lumpur, Malaysia, 2016.

2. T. R. Raddo, A. L. Sanches, I. T. Monroy, and B.-H. V. Borges, "Multirate IP traffic Transmission in flexible access networks based on optical FFHCDMA" in Proc. IEEE International Conference on Communications, Kuala Lumpur, Malaysia, 2016.

3. T. R. Raddo, A. L. Sanches, B.-H. V. Borges, and I. T. Monroy, "Throughput performance analysis of multirate, multiclass S-ALOHA OFFH-CDMA packet networks," in Proc. IEEE International Conference on Transparent Optical Networks, Budapest, Hungary, 2015.

4. T. R. Raddo, A. L. Sanches, J. V. dos Reis Jr., and B. -H. V. Borges, "Throughput evaluation of multirate, multiservice 2-D OCDMA packet networks," in Proc. IEEE International Conference on Transparent Optical Networks, Graz, Austria, 2014. 
5. T. R. Raddo, A. L. Sanches, J. V. dos Reis, Jr, and B. -H. V. Borges, "Multiservice, multirate IP transmission over OCDMA network," in Proc. IEEE International Conference on Transparent Optical Networks, Cartagena, Spain, 2013.

6. T. R. Raddo, A. L. Sanches, J. V. dos Reis, Jr, and B. -H. V. Borges, "Influence of the MAI Distribution over the BER Evaluation in a Multirate, Multiclass OOC-OCDMA System," in Proc. OSA Access Networks and In-house Communications, Toronto, Canada, 2011, paper ATuB5.

\section{Publications not Related to this Thesis}

\section{Peer-reviewed Journal Articles}

1. T. R. Raddo, A. Jurado-Navas, A. L. Sanches, J. M. Garrido-Balsells, J. J. Vegas Olmos, B.-H. V. Borges, and I. Tafur Monroy “Multiservice multirate free-Space optical CDMA communications system for flexible access networks", 2017, to be submitted.

2. A. Jurado-Navas, T. R. Raddo, J. M. Garrido-Balsells, B.-H. V. Borges, J. J. Vegas Olmos, and I. Tafur Monroy, "Hybrid optical CDMA/FSO communications network under spatially correlated gamma-gamma scintillation", OSA Optics Express, vol. 24, no. 15, pp. 16799-16814, July 2016.

3. A. L. Sanches, T. R. Raddo, J. V. dos Reis Jr., and B.-H. V. Borges, "Performance analysis of single and multirate FFH-OCDMA networks based on PSK modulation formats," IEEE/OSA J. Opt. Commun. and Netw., vol. 7, no. 11, pp. 1084-1097, November 2015.

4. J. V. dos Reis Jr., T. R. Raddo, A. L. Sanches, and B.-H. V. Borges, “Fuzzy logic control for the mitigation of environmental temperature variations in OCDMA networks," IEEE/OSA J. Opt. Commun. and Netw., vol. 7, no. 5, pp. 480-488, May 2015.

\section{International Conferences Proceedings Articles}

1. A. Jurado-Navas, T. R. Raddo, A. L. Sanches, J. M. Garrido-Balsells, B.-H. V. Borges, J. J. Vegas Olmos, and I. Tafur Monroy, “Asynchronous free-space optical CDMA communications system for last-mile access networks", in 
Proc. OSA Propagation Through and Characterization of Atmospheric and Oceanic Phenomena, Washington, District of Columbia, USA, 2016.

2. A. L. Sanches, T. R. Raddo, J. V. dos Reis Jr., L. H. Bonani, and B.-H. V. Borges, "BER performance improvement in FFH-OCDMA networks with BPSK modulation format," in Proc. IEEE International Conference on Transparent Optical Networks, Trento, Italy, 2016.

3. A. L. Sanches, T. R. Raddo, J. V. dos Reis Jr., and B.-H. V. Borges, and I. T. Monroy, "Multirate FFH-OCDMA networks based on coherent advanced modulation formats," in Proc. IEEE International Conference on Transparent Optical Networks, Budapest, Hungary, 2015.

4. J. V. dos Reis Jr., T. R. Raddo, A. L. Sanches, and B.-H. V. Borges, "Comparison between Mamdani and Sugeno fuzzy inference systems for the mitigation of environmental temperature variations in OCDMA-PONs," in Proc. IEEE International Conference on Transparent Optical Networks, Budapest, Hungary, 2015.

5. A. L. Sanches, T. R. Raddo, J. V. dos Reis Jr., and B. -H. V. Borges, “Highly efficient FFH-OCDMA packet network with coherent advanced modulation formats," in Proc. IEEE International Conference on Transparent Optical Networks, Graz, Austria, 2014.

6. J. V. dos Reis Jr., T. R. Raddo, A. L. Sanches, and B. -H. V. Borges, “Mitigation of environmental temperature variation effects using fuzzy systems and source-matched spreading codes for OCDMA networks," in Proc. IEEE International Conference on Transparent Optical Networks, Graz, Austria, 2014.

7. G. Gimenes, H. Gualdron, T. R. Raddo, and J. F. Rodrigues, Jr, "Supervisedlearning link recommendation in the DBLP co-authoring network," in Proc. IEEE PerCom 2nd International Workshop on Social and Community Intelligence, Budapest, Hungary, 2014.

8. L. Galdino, T. R. Raddo, A. L. Sanches, L. H. Bonani, and E. Moschim, "Performance comparison of hybrid 1-D WDM/OCDMA and 2-D OCDMA towards future access network migration scenario," in Proc. Proc. IEEE International Conference on Transparent Optical Networks, Coventry, England, 2012.

9. T. R. Raddo, A. L. Sanches, J. V. dos Reis, Jr, and B. -H. V. Borges, "Performance Evaluation of a Multirate, Multiclass OCDM/WDM Optical 
LIST OF PUBLICATIONS

Packet Switch," in Proc. IEEE International Microwave and Optoelectronics Conference, Natal, Brazil, 2011. 


\section{List of Acronyms}

\begin{tabular}{|c|c|}
\hline 1-D & One-Dimensional \\
\hline 2-D & Two-Dimensional \\
\hline 3-D & Three-Dimensional \\
\hline AON & Active Optical Network \\
\hline APD & Avalanche Photodiode \\
\hline ASK & Amplitude Shift Keying \\
\hline AWG. & Arrayed waveguide grating \\
\hline BER & Bit Error Rate \\
\hline BPSK & Binary-Phase-Shift-Keying \\
\hline CCD & Charge Coupled Device (Camera) \\
\hline CDMA & Code-Division Multiple-Access \\
\hline CLT & Central Limit Theorem \\
\hline DS & Direct-Sequence \\
\hline EEL & Edge Emitting Laser \\
\hline EP & Elliptically Polarized \\
\hline FBG & Fiber Bragg Grating \\
\hline FE & Frequency Encoded \\
\hline FEC & Forward Error Correction \\
\hline FFH & Fast Frequency Hopping \\
\hline FH & Frequency-Hopping \\
\hline FIFO & First In First Out \\
\hline FPI & Fabry-Perot Interferometer \\
\hline FSO & Free-Space Optics \\
\hline FTTB & Fiber To The Building \\
\hline FTTC & Fiber To The Curb \\
\hline
\end{tabular}


FTTH Fiber To The Home

FTTx Fiber To The $x$

GP Grassberger-Procaccia

HDTV High-Definition Television

HF High Frequency

IM-DD Intensity-Modulation Direct-Detection

IP Internet Protocol

IPTV Internet Protocol Television

LED Light-Emitting Diode

LI .. Light Intensity

LF $\ldots \ldots \ldots \ldots \ldots$ Low Frequency

LLE $\ldots \ldots \ldots \ldots \ldots$ Largest Lyapunov Exponent

LP $\ldots \ldots \ldots \ldots$. Linearly Polarized

MZI .............. Mach-Zehnder interferometer

MAC . Media Access Control

MAI Multiple-Access Interference

MBGs Multiple Bragg Gratings

MWML-OOC Multi-Weight Multi-Length OOC

MWOOC Multiple-Wavelength Optical Orthogonal Code

OCDM Optical Code-Division Multiplexing

OCDMA Optical Code-Division Multiple-Access

OCDM/WDM Optical Code-Division Multiplexing/Wavelength-Division

Multiplexing

OCFHC/OOC One-Coincidence Frequency-Hopping Code/Optical

Orthogonal Code

ODLs Optical Delay Lines

OFFH Optical Fast Frequency Hopping

OOC Optical Orthogonal Code

OOK On-Off Keying 
OPS

Optical Packet Switch

OSA Optical Spectrum Analyzer

PDF Probability Density Function

PER . Packet Error Rate

PLP Packet Loss Probability

PON Passive Optical Network

PS . Polarization Switching

PSK Phase-Shift Keying

QPSK Quadrature-Phase-Shift-Keying

QD-VCSELS Quantum-dot Vertical-Cavity Surface-Emitting Lasers

QoE Quality of Experience

QoS Quality of Service

QW-VCSELS Quantum-well Vertical-Cavity Surface-Emitting Lasers

RF . Radio Frequency

RS Reed-Solomon

RTT Round-Time Trip

S-ALOHA Slotted ALOHA

SMF Single Mode Fiber

SHD Super High-Definition

TDM Time-Division Multiplexing

TDMA Time-Division Multiple-Access

TDM/OCDMA . Time-Division Multiplexing/Optical Code-Division

Multiple-Access

VCSELS Vertical-Cavity Surface-Emitting Lasers

VoD Video-on-Demand

WDM Wavelength-Division Multiplexing

WDMA Wavelength-Division Multiple-Access

WDM/OCDMA Wavelength-Division Multiplexing/Optical Code-Division

Multiple-Access 



\section{List of Symbols}

$A B_{j}$

$b$

B

$b_{1, j^{\prime}}$

$B E R\left(j^{\prime}\right)$

$B_{j}$

$B_{j^{\prime}}$

$b_{u, j}(t)$

$c$

C

$c_{f, j}$

$c_{F_{j}, j}$

$C_{j}$

$C_{J}$

$C_{u, j}(t, f)$

$D A_{-} C_{j}$

$E A_{-} C_{J}$

$f\left(m_{j^{\prime}}\right)$ arrivals

F

F

$F_{j}$

$f_{M}(m)$ arrivals

$f(t)$

$G_{j}$

$G_{j^{\prime}}$

F
Average bit rate of class- $j$

A random chosen packet

Data rate transmission

Reference bit of the desired user

. Bit error rate of the desired $j^{\prime}$ class

. Number of class- $j$ codes assigned to each user

. Multicode factor

. Baseband signal of the $u$ th user in the $j$ th class

. Speed of light

. Total code sets available

. The fth codeword of the jth class

. The last available codeword of the $j$ th class

. Code set of the $j$ th class

. The last available code set in the system

Hopping pattern of the $u$ th user in the $j$ th class

. Decoder array that includes all decoders of users class $j$

. Encoder array that includes all encoders of users class $j$

. Steady-state probability distribution of composite packets

Total number of available wavelengths

.. Total number of packets in the system

. Total number of packets in class $j$

. . General steady-state probability distribution of composite

. Code mask

. Offered load of class- $j$

. Offered load of class- $j^{\prime}$ 
H

$H_{j^{\prime}}$

$I_{i}$

$\mathrm{I}_{\mathrm{i}, \mathrm{m}, \mathrm{i}}$

class in $m$ th wavelength

$I_{j^{\prime}}$

$I_{\left(j=j^{\prime}, u\right)}^{1, j^{\prime}}$

$I_{\left(j>j^{\prime}, u\right)}^{1, j^{\prime}}$ user's code length

$I_{\left(j<j^{\prime}, u\right)}^{1, j^{\prime}}$

MAI from users with code lengths shorter than the desired user's code length

j

$K_{j}$

L

$L$

$L_{J}$

$L_{s}$

$m_{j} \in\left\{0,1, \ldots, U_{j}\right\} \ldots \ldots$ Number of simultaneous class- $j \mathrm{~s}$ transmitting users

$m_{j^{\prime}} \in\left\{0,1, \ldots, U_{j^{\prime}}\right\} \ldots$. Number of simultaneous class- $j^{\prime}$ transmitting users

$M B_{j}$

$M_{I_{j^{\prime}}}(t)$

$M_{I_{j^{\prime}}}^{\prime}(0)$

$M_{I^{\prime}}^{\prime \prime}(0)$

neff

$N_{j}$

$p$

$P \quad \ldots \ldots \ldots \ldots \ldots$. Prime number representing the number of available wavelengths employed in the code construction

Packet length in bits

. Length of class- $j$ ' packet expressed in bits

Set of left packets from $I_{i, m, j}$

. Set of packets arrived in $i$ th output fiber, forwarded to $j$ th

Total MAI of the system generated on the desired class

MAI from users of same code lengths that desired user

MAI from users with code lengths longer than the desired

The desired user class

. Code cardinality in class- $j$

Code lengt

. Code length of $j$ th user class in the system

. Code length of last user class in the system

. Spacing between two adjacent Bragg gratings

. Maximum bit rate of class- $j$

. Moment-generating function of total interference

. First moment of the moment-generating function

. Second moment of the moment-generating function

fff $\ldots \ldots \ldots \ldots$ Effective index

. Number of interfering users in relation to the desired user

$\ldots \ldots \ldots \ldots$ Offered traffic

............................. Code 
$\mathcal{P}$ Probability of interference of a multirate, multiservice system

$P\left(b_{1, j^{\prime}}=0\right) \quad \ldots \ldots \ldots$ Transmission probabilities of bit " 0 "

$P\left(b_{1, j^{\prime}}=1\right) \ldots \ldots \ldots$ Transmission probabilities of bit " 1 "

$P_{C}\left(j^{\prime}\right) \ldots \ldots \ldots \ldots$ Packet correct probability

$P($ error $\mid 0) \quad \ldots \ldots \ldots$ Probability of false alarm

$P($ error $\mid 1) \quad \ldots \ldots \ldots$ Probability of false dismissal

$p_{j j^{\prime}} \quad \ldots \ldots \ldots \ldots \ldots$ Probability of hit between a code from class $j$ and a code from class $j^{\prime}$

$p_{j^{\prime} j^{\prime}} \quad \ldots \ldots \ldots \ldots \ldots$ Probability of hit between a code from class $j^{\prime}$ and a code from class $j^{\prime}$

$P\left(Z \geq \mu \mid b_{1, j^{\prime}}=0\right) \ldots$. Probability that a bit " 0 " is transmitted and $\mathrm{Z}$ reaches or exceeds the detection threshold

$P\left(Z<\mu \mid b_{1, j^{\prime}}=1\right) \ldots$. Probability that a bit " 1 " is transmitted and $Z$ does not exceed the detection threshold

$Q(\cdot) \quad \ldots \ldots \ldots \ldots \ldots, Q$-function

$r(t) \quad \ldots \ldots \ldots \ldots \ldots$ Signal after correlation between a specific code and its mask

$r(t, f) \quad \ldots \ldots \ldots \ldots \ldots$ Broadcasted received signal

$S F_{A} \quad \ldots \ldots \ldots \ldots \ldots$ Switching Fabric

$S F_{i} \quad \ldots \ldots \ldots \ldots \ldots$ ith Switching Fabric

$s(t) \quad \ldots \ldots \ldots \ldots \ldots$ Code Sequence signature

$S_{u, j}(t, f) \quad \ldots \ldots \ldots \ldots$ Transmitted signal of the $u$ th user in the $j$ th class

T ........... Total number of users accommodated in the network

$T_{b} \ldots \ldots \ldots \ldots \ldots \ldots$ Bit period

$T_{c} \ldots \ldots \ldots \ldots \ldots$ Chip period

$T_{j} \ldots \ldots \ldots \ldots \ldots$ Bit period of $j$ th user class

U .......... Total number of users

UC ........... Total number of active codes in the network

$U_{j} \ldots \ldots \ldots \ldots \ldots$ Total number of users in class $-j$

$U_{j^{\prime}} \ldots \ldots \ldots \ldots \ldots$ Total number of users in the desired class- $j^{\prime}$ 
$W \ldots \ldots \ldots \ldots \ldots$ Code weight

$W C_{r} \quad \ldots \ldots \ldots \ldots \ldots$ th Wavelength converter

$W_{R} \quad \ldots \ldots \ldots \ldots$ Total number of available wavelength converters

$W_{j} \quad \ldots \ldots \ldots \ldots \ldots$ Code weight of the users' interfering class

$W_{j^{\prime}} \ldots \ldots \ldots \ldots \ldots$ Code weight of the users' desired class

$Z_{x, x} \ldots \ldots \ldots \ldots$ Autocorrelation function

$Z_{x, y} \quad \ldots \ldots \ldots \ldots$ Cross-correlation function

$\beta\left(j^{\prime}\right) \ldots \ldots \ldots$ General packet throughput

$\beta_{P O I}\left(j^{\prime}\right) \ldots \ldots \ldots \ldots$ Packet throughput under the Poisson approach

$\beta_{\text {bin }}\left(j^{\prime}\right) \ldots \ldots \ldots$ Packet throughput under the binomial approach

$\beta_{\text {mar }}\left(j^{\prime}\right) \ldots \ldots \ldots$ Packet throughput under the Markov approach

$\lambda_{a} \ldots \ldots \ldots \ldots \ldots$ Out-of-phase autocorrelation peak

$\lambda_{c} \ldots \ldots \ldots \ldots \ldots$ Cross-correlation peak

$\lambda_{F} \ldots \ldots \ldots \ldots \ldots$ Last available wavelengths in the OFFH system

$\lambda_{m} \ldots \ldots \ldots \ldots \ldots \ldots$ th wavelength from $M$ available wavelengths

$\mu \quad \ldots \ldots \ldots \ldots \ldots$ Threshold level of the decision device

$\mu_{\text {opt }} \quad \ldots \ldots \ldots \ldots \ldots$ Optimum threshold level of the decision device

$\eta \quad \ldots \ldots \ldots \ldots \ldots$ Mean of the MAI

$\xi \ldots \ldots \ldots \ldots \ldots$ Independent Bernoulli random variable

$\sigma \quad \ldots \ldots \ldots \ldots \ldots$ Square root of the variance

$\sigma^{2} \quad \ldots \ldots \ldots \ldots \ldots$ Variance of the MAI

$\tau_{u, j} \ldots \ldots \ldots \ldots \ldots$ Time delay associated with the $u$ th signal of the $j$ th class

$\lfloor.\rfloor \ldots \ldots \ldots \ldots \ldots$ Denotes the integer part 


\section{List of Figures}

Figure 1.1. Schematic representation of possible FTTH solutions for three different scenarios. a) a point-to-point network with $U$ optical fibers and $2 U$ transceivers. b) a passive optical network with only one feeder fiber and $U$ transceivers featuring the most efficient solution. c) a switched network with one feeder fiber and $2 \mathrm{U}+2$ transceivers. CO: central office. P: passive. A: active. Each house represents a user $\in$ $\{1,2, \ldots, U\}$. Picture adapted from [6].

Figure 1.2. Multiple access schemes. $U$ is the total number of users, $\lambda_{1}$ is the wavelength \#1, and code 1 is the code sequence \#1. a) WDMA (the colors distinguish the wavelengths), each user transmits in a range of wavelength simultaneously, and b) OCDMA (the colors distinguish the codes), each user transmits simultaneously using its own code sequence, occupying all frequency range available. Figure adapted from [13]. . .7

Figure 1.3. Bandwidth required to represent the information signal and bandwidth utilized by the spread spectrum system for the transmission.

Figure 1.4. Viable architecture of a flexible OCDMA network with support to dynamic bandwidth allocation and multiservice transmissions. Each user class has its own code parameters specification. Each user has a unique code. In this example, link direction, network broadcast and packet switching concept are not illustrated for the sake of simplicity. Picture taken from [39].

Figure 2.1. Scheme of the encoding process of a user's data bit into an OCDMA code sequence formed by several short pulses. a) Short pulse representing the generated data bit of user class-1. b) Data bit stream of user class-1. c) Encoded short pulses representing the code sequence of class-1. d) Data bit stream of user class-2. e) Encoded short pulses representing the code sequence of class- 1 . Note that $T_{c}$ is constant. For the sake of simplicity, only the incident (not-encoded) generated data bit for class-1 user is shown in (a).

Figure 2.2. 1-D MWML-OOC encoding scheme based on time spreading. a) A wavelength-independent short pulse corresponding to data bit " 1 " is sent to the encoder. b) Possible encoder scheme based on ODLs, splitter and combiner. c) Encoded 1-D MWML-OOC signal. Picture adapted from [1].

Figure 2.3. 1-D MWML-OOC decoding scheme based on intensity correlation. a) Received signal is sent to the decoder. b) Possible decoder scheme based on ODLs, splitter and combiner. c) Autocorrelation signal plus interference from other users. d) Cross-correlation signal. Picture adapted from [1].

Figure 2.4. Frequency hopping technique. a) Time and frequency occupancy of frequency-hopping (FH) signal. b) Time and frequency occupancy of fast frequencyhopping (FFH) signal. 


\section{LIST OF FIGURES}

Figure 2.5. Example of 2-D OOC. a) All network's users assigned with regular 2-D OOC codes with equal code-weight and -length. b) Distinct users assigned with codes of different weight and code to achieve multiservice, multirate transmissions

Figure 2.6. 2-D MWML-OOC encoding scheme based on time and frequency spreading. a) A broadband short pulse corresponding to data bit " 1 " is sent to the encoder. b) Possible encoder scheme based on ODLs, demultiplexer (DEMUX) and multiplexer (MUX). c) Encoded signal. Picture adapted from [1].

Figure 2.7. 2-D MWML-OOC decoding scheme based on time and frequency spreading. a) Transmitted encoded signal is sent to the decoder. b) Possible decoder based on ODLs, demultiplexer (DEMUX) and multiplexer (MUX). c) Output autocorrelation signal. d) Output cross-correlation signal. Picture adapted from [1].

Figure 2.8. Example of FFH-based multilength codes. a) Regular OFFH scheme assigning codes with the same length for all network's users. b) Multilength OFFH scheme assigning larger codes to low-rate users and shorter codes to high-rate users.

Figure 2.9. Possible implementation of the FFH scheme based on MBGs. a) A broadband short pulse corresponding to data bit " 1 " is sent to the encoder. b) The MBGs reflect the signal encoding simultaneously in sequential time slots and disjoint wavelength subbands according to the respective wavelengths in the Bragg grating by the MBGs-based encoder. c) The encoded time-frequency sequence is ready to be transmitted. d) MBGs-based decoder with gratings placed in the reversed order. e) Autocorrelation signal plus interference and cross-correlation signals. SMF: single mode fiber. Picture adapted and based on a setup of single rate networks from [76].

37

Figure 2.10. Example of the MAI contribution on the desired user class \#1 in a flexible OCDMA network with four interfering users.

Figure 2.11. Scheme of the multirate OCDMA network connecting all J-class users in a star topology via optical fibers and a passive star coupler. Each user class has its own code parameters specifications. A two-class network is considered for the sake of space. a) Multilength code technique, where class-j' has shorter code than class-J. b) Multicode technique, where to each class-j' user two codes are assigned whereas to each class-J user only one code is assigned. Picture taken from [109]. . .41

Figure 2.12. Example of the packet traffic transmission for both users' classes employing the multicode technique. Picture adapted from [91].

Figure 2.13. Block diagram of the transmitters considering the important stages for both multirate techniques. a) Multilength code technique employing only a single encoder. b) Multicode technique employing several encoders for a single user. Picture adapted from [90].

Figure 2.14. Block diagram of the receivers considering the important stages for both multirate techniques. a) Multilength code technique employing only a single decoder. 
b) Multicode technique employing several decoders for a single user. Picture adapted from [90].

Figure 3.1: Block diagram of the flexible OCDMA network with support to multiservice, multirate transmissions, where users are connected in a star topology via optical fibers and passive star coupler/splitter. Only the more important stages are shown. C: coupler, S: splitter.

Figure 3.2: Block diagram of the proposed IP traffic architecture based on OCDMA with support to multiservice, multirate transmissions. Picture taken from [101]......55

Figure 3.3: Block diagram of the flexible OCDMA packet network with users arranged into classes in accordance with their QoS and rate transmission requirements. Each user class has its own code's parameters specifications. The codes' pulses are colourless in the 1-D network case.

Figure 3.4: Example of the S-ALOHA OCDMA packet model for a three-class multirate network, where class- 1 ( $\mathrm{L}_{1}=4$ and $\mathrm{H}_{1}=4$ bits), class- 2 ( $\mathrm{L}_{2}=5$ and $\mathrm{H}_{2}=3$ bits), and class $-3\left(\mathrm{~L}_{3}=8\right.$ and $\mathrm{H}_{3}=2$ bits) have the same packet length, $\mathrm{T}_{\mathrm{p} 1}=\mathrm{T}_{\mathrm{p} 2}=\mathrm{T}_{\mathrm{p} 3}=\mathrm{T}_{\mathrm{p}}$. Picture taken from [39].

Figure 3.5: Packet flow model of the S-ALOHA OCDMA network. Picture adapted from [102].

Figure 4.1. BER versus the number of simultaneous users for a conventional singlerate OCDMA network with $\mathrm{W}=7, \mathrm{~L}=1000$ (or $\mathrm{L}=2000$ ), and $\mathrm{U}=20$. Multirate BER formalism considering only a single user class (symbols) and conventional single rate formalism (solid lines)...... .76

Figure 4.2: BER as a function of simultaneous users for a three classes MWML-OOC system. The MAI distributions are Poisson (solid lines) [42] and binomial (dotted lines) [96]. The subscripts 1, 2 and 3 refer to the different classes investigated. ........78

Figure 4.3: Packet throughput versus the offered load of a conventional single-rate single-service OCDMA network for validation purposes. .79

Figure 4.4: Packet throughput of the desired class versus the offered load of the desired class for a two-class 1-D MWML-OOC OCDMA packet network employing binomial (squares), Poisson (triangles), and Markov (circles) approaches. . .80

Figure 4.5: Packet throughput performance of a two-class 1-D MWML-OOC OCDMA network employing binomial (squares), Poisson (triangles), and Markov (circles) approaches. a) Throughput of class-1 versus the offered load of class-1 (solids). b) Throughput of class-2 versus the offered load of class-2 (dashed). At $\mathrm{G}_{2}=8$ and $\mathrm{G}_{2}=25$ the Poisson approach underestimates the throughput by around $75 \%$ and $150 \%$, respectively.

Figure 4.6: Packet throughput performance of a two-class 2-D MWML-OOC OCDMA packet network employing binomial (squares), Poisson (triangles), and Markov (circles) approaches. a) Throughput of class-1 versus the offered load of class-1 (solids). b) Throughput of class-2 versus the offered load of class-2 (dashed). 


\section{LIST OF FIGURES}

Figure 4.7: Optimum detection threshold with $L 1=12, L 2=6, U 1=17, U 2=6$, and $F=$ 29. The BER of high rate users and low rate users are minimized by choosing threshold values of 6 and 12, respectively . .86

Figure 4.8: BER versus the number of simultaneous class-1 users for a two-class network with $L 1=12, U 1=17, L 2=6, U 2=6$ and $F=29$. The number of users in class-1 is varied from 1 to 17 . Binomial approach (solid lines) and gaussian approximation (dashed lines). .88

Figure 4.9: BER versus the number of simultaneous class-1 users for a two-class network with $L 1=12, L 2=6$, and $F=29$. The number of class- 1 users is varied from 1 to 17 . Class-2 has only 4 users, $U 2=4$. Binomial approach (solid lines) and gaussian approximation (dashed lines). .89

Figure 4.10: BER versus the number of simultaneous class-1 users for a two-class network with $L 1=12, L 2=6$, and $F=29$. The number of class- 1 users is varied from 1 to 17 . The number of class- 2 users is either $U 2=4$ or $U 2=6$. Only results for the binomial approach are shown.

Figure 4.11: BER performance of the desired user-class versus the simultaneous number of class- 1 users' codes for a two-class network and for both multicode (solid lines) and multilength code techniques (dotted lines). . .91

Figure 4.12: Packet throughput of class- 1 versus the offered load of class- 1 for a twoclass network for both multicode and multilength code techniques.

Figure 4.13: Packet throughput of class-2 versus the offered load of class-2 for a twoclass network for both multicode and multilength code techniques. .92

Figure 4.14: PER performance for all classes versus number of class-3 simultaneous users for a three-class 1-D OCDMA IP packet network. The channel utilization is considered either 0.4 (solid lines) or 0.8 (dashed lines).

Figure 4.15: PER performance versus the number of active class-2 users for a twoclasses 1-D OCDMA IP packet network. The number of class 1 users is fixed to $U 1=$ 10. The channel utilization is varied from 0.2 to 1 ( 0.2 spacing) for both classes accordingly. a) Class-1 results. b) Class-2 results.

Figure 4.16: Decoder BER performance for Class-1 (dashed line) and Class-2 (solid line) of a flexible IP OCDMA packet network based on 2-D FFH scheme. The number of users in class-2 is varied from 1 to 17. . .96

Figure 4.17: Total BER performance of a flexible IP OCDMA packet network based on 2-D FFH scheme for different values of channel utilization and $\mathrm{H}=12000$ bits. a) BER of class- 1 versus number of simultaneous class- 2 users. b) BER of class- 2 versus number of simultaneous class-2 users. The channel utilization is varied from 0.2 to 1 (0.2 spacing) for both classes. The dotted horizontal line indicates the standard BER limit region.

Figure 4.18: PER performance of a flexible IP OCDMA packet network based on 2-D FFH scheme for different values of channel utilization and $\mathrm{H}=12000$ bits. a) PER of 
class-1 versus number of simultaneous class-2. b) PER of class-2 versus number of simultaneous class- 2 users. The channel utilization is varied from 0.2 to 1 ( $0.2 \mathrm{spacing}$ ) for both classes.

Figure 4.19: Throughput performance of a flexible IP OCDMA packet network based on 2-D FFH scheme for different values of channel utilization and $\mathrm{H}=12000$ bits. a) Throughput of class-1 versus the offered load of class-1. b) Throughput of class-2 versus the offered load of class-2. The channel utilization is varied from 0.2 to 1 (0.2 spacing) for both classes.

Figure 4.20: a) Throughput of class-1 versus the offered load of class-1 for a two-class flexible OCDMA network. b) Throughput of class-2 versus the offered load of class2 for a two-class flexible OCDMA network. 100

Figure 4.21: a) Delay of class-1 versus the offered load of class-1 for a two-class network employing binomial, Poisson, and Markov approaches. b) Delay of class-2 versus the offered load of class- 2 for a two-class flexible OCDMA network for three different approaches, namely the binomial (squares), Poisson (triangles) and Markov chain (circles) approaches.

Figure 4.22: a) Delay of class-1 versus the throughput of class- 1 for a two-class flexible OCDMA network for three different approaches, namely the binomial (squares), Poisson (triangles) and Markov chain (circles) approaches. b) Delay of class-2 versus the throughput of class-2 for a two-class flexible OCDMA network for three different approaches.

Figure 5.1. Schematic designs of classical VCSEL and edge-emitting laser structures. a) Scheme of a VCSEL with emission perpendicular to the wafer plane and with an output beam on the vertical axis. b) Scheme of an EEL structure with emission perpendicular to the wafer plane on the side of the structure. Figure adapted from [128]. 109

Figure 5.2. Distinct types of polarization switching events. Polarization-resolved optical output power versus the injection current for three different substrate temperatures. a) $\mathrm{T}=10^{\circ} \mathrm{C}$ featuring a PS of type $\left.\mathrm{I}, \mathrm{b}\right) \mathrm{T}=15^{\circ} \mathrm{C}$ featuring a double PS event, type I followed by type II, (c) $\mathrm{T}=55^{\circ} \mathrm{C}$ featuring a PS of type II. Picture taken from [130].

Figure 5.3. Polarization resolved LI-curves for increasing currents and statistics of the mode hopping dynamics. a) Polarization resolved-LI curve for increasing injection currents. The laser emits on a LP state from threshold to $\mathrm{I}_{\mathrm{A}}$ and then on the EP state for $\mathrm{I}_{\mathrm{A}}<\mathrm{I}<\mathrm{I}$ s. Random like hopping dynamics between two EP states are observed for Is $<$ I $<I_{R}$, and the laser emits in a two-mode emission for I $>I_{R}$. b) Evolution of the average dwell-time for the mode-hopping dynamics with an inset showing the timeseries of the random-like hopping for polarization projection at $45^{\circ}$ concerning the linear polarization at threshold. Pictures taken from [138]. 112

Figure 5.4. Picture of the full experimental setup used to exploit the complex polarization dynamics of a QW-VCSEL subjected to mechanical stress. L: lens, $\lambda / 2$ : 


\section{LIST OF FIGURES}

half wave plate, OI: optical isolator, M1: mirror \#1, MM Fiber: multimode fiber, IRIS: iris diaphragm, M2: mirror \#2, CCD camera, objective and VCSEL.

Figure 5.5. Schematic of the experimental setup used to exploit the complex polarization dynamics of a QW-VCSEL subjected to mechanical stress. The full line represents the light path starting from the QW-VCSEL until reaches one of the twopossible multimode fiber configuration. a) setup configuration used for general characterization of the QW-VCSEL with the mirror (M1) down (dotted line). b) setup configuration used to exploit the influence of the second higher-order mode (dashed line). L1: lens \#1, $\lambda / 2$ : half wave plate, L2: lens \#2, OI: optical isolator, L3: lens \#3, M1: mirror \#1, L4: lens \#4, OB: Objective, MMFiber: multimode fiber, IRIS: iris diaphragm, M2: mirror \#2 and VCSEL

Figure 5.6. Custom-made holder based on the mechanical stress technique. The holder comprises an aluminium plate with a central hole to place the already packaged QW-VCSEL in combination with a pressing plate fixed with two screws used as a lid. By placing a steel rod behind the laser package, the pressure applied by the pressing plate induces anisotropic strains in the device cavity.

115

Figure 5.7. Schematic design and suggested dimensions of the custom-made VCSEL holder for the application of the mechanical stress technique. The packaged VCSEL is placed behind the central hole of the lid and a steal rod (not illustrated) is placed between the VCSEL and the metal plate. Pressure can then be applied on the metal lid and adjusted using the M2 screws (not illustrated) on each side of the device. A thermistor is placed into the metal plate's right side hole to control the device temperature.

Figure 5.8. Polarization resolved LI-curves for increasing injection current and for polarization projection at $0^{\circ}, 90^{\circ}, 45^{\circ}$ and $-45^{\circ}$ with respect to the LP at threshold. No PS events are observed. a) Stress level \#3, and b) stress level \#5. .117

Figure 5.9. Polarization resolved LI-curves for increasing injection current and for polarization projection at $0^{\circ}, 90^{\circ}, 45^{\circ}$ and $-45^{\circ}$ with respect to the LP at threshold. A double PS event can be observed in both pictures. a) A medium level of mechanical stress (\#8) is loaded onto the QW-VCSEL. b) A high level of stress (\#9) is loaded onto the QW-VCSEL.

Figure 5.10. Dynamic measurements. Evolution of the Radio Frequency spectrum for increasing injection current for polarization at $0^{\circ}$ a) and at $45^{\circ} \mathrm{b}$ ). Panel c) shows the zoomed-in plot highlighted by the red rectangle in (a) showing the featureless lowfrequency contribution corresponding to the mode-hopping dynamics. In d) to g), we show time-series recorded for polarization at $90^{\circ}$ showing random-like hopping between polarization eigenmodes with a significant decrease of residence time for increasing injection current at: 6.15, 6.27, 6.39 and $6.44 \mathrm{~mA}$ respectively.

Figure 5.11. Mode hopping statistics. a) Average residence time when considering all jumps. The red line gives a linear fit. b) Average residence time for the upper (red) 
and lower (blue) levels. The upper and lower levels are defined for a time-series recorded at $0^{\circ}$ polarization. c) Number of jumps considered for each point.

Figure 5.12. Estimation of the Largest Lyapunov Exponent for increasing injection current using Wolf's algorithm.

Figure 5.13. Results of the GP algorithm. In both panels, we show the evolution of the curves for increasing segment length D. Panel a) shows the evolution of the correlation integral $C_{D}$ - i.e. the average number of neighbouring points in the sphere of radius $r$. In panel $b$ ) we show the derivative $d\left(\ln \left(C_{D}\right)\right) / d(\ln (r))$ that leads to a converging correlation dimension for increasing length of segment D. Such convergence is clearly obtained around $\ln (\mathrm{r})=6.3$ where the curves reach a plateaus around 2.04 . 123

Figure 5.14. Polarization resolved output power for increasing injection current and for projection at $0^{\circ}, 90^{\circ}, 45^{\circ}$ and $-45^{\circ}$ with respect to the LP at threshold. A relatively high level of strain is applied onto the QW-VCSEL device and a double PS event along with polarization chaos is observed. The two insets show the spatial beam profile for polarization at $0^{\circ}$ and $90^{\circ}$ and for $6 \mathrm{~mA}$, confirming the existence of a $2^{\text {nd }}$ order mode at high current levels (bottom right inset).

Figure 5.15. Polarization resolved light intensity curves for increasing injection current and for polarization projection at $0^{\circ}, 90^{\circ},+45^{\circ}$ and $-45^{\circ}$ with respect to the LP at threshold. An iris diaphragm almost fully closed was used to spatially filter out the second higher-order transverse mode. The scale of the transverse intensity profile of the beam is given in a.u.. It is observed a double PS event along with polarization chaos measuring the fundamental mode only. The two insets show the spatial beam profile for polarization at $0^{\circ}$ and $90^{\circ}$ for $6 \mathrm{~mA}$, confirming the predominance of the fundamental mode.

Figure 5.16. Polarization resolved optical spectra for projection at $0^{\circ}$ and for injection current at $6 \mathrm{~mA}$. a) Without the iris diaphragm. b) With the iris diaphragm included in the setup. 127

Figure 5.17. Polarization resolved optical spectra for projection at $90^{\circ}$ and injection current at $6 \mathrm{~mA}$. a) Without the iris diaphragm. b) With the iris diaphragm included in the setup.

Figure 5.18. Frequency resolved LI-curves. $(a, b)$ typical two-mode optical spectra recorded at $4 \mathrm{~mA}$ for polarization at $0^{\circ} \mathrm{a}$ ), and $90^{\circ} \mathrm{b}$ ). The horizontal dashed lines indicate the threshold above which spectral data are integrated to estimate the power of each mode. (c, d) Frequency resolved LI curves for polarization at $0^{\circ}$ c), and $90^{\circ}$ d): the black (red) solid line with crosses represents the fundamental mode while the orange (blue) solid line with circles represents the second order mode. The vertical dash-dotted line indicates the threshold for the second order mode, i.e. about $3 \mathrm{~mA}$.

Figure 5.19. Frequency resolved LI-curves for polarization projection at a) $0^{\circ}$, b) $90^{\circ}$, c) $45^{\circ}$, and d) $-45^{\circ}$. For each panel the projection orientation is also indicated by the 


\section{LIST OF FIGURES}

red arrow in the black circle. In all panels, the blue line with crosses shows the evolution of the fundamental mode while the orange line with circles represents the second order mode. The inset in panel (b) shows a zoom around the switching point delimited by the red rectangle.

.129

Figure 5.20. Schematic orientation view of the elliptically polarized states. a) Typical polarization chaos time-series showing a random-like hopping between two elliptically polarized states EP1 and EP2 highlighted in blue and red, respectively. The orientation of these two states is shown with respect to the laser eigenmodes for a typical case in QD VCSEL and for the stressed VCSEL described here in b) and c), respectively

.130 


\section{Bibliography}

[1] H. Yin and D. J. Richardson, Optical code division multiple access communication networks: theory and applications. Tsinghua University Press, 2008.

[2] 'Tactile Internet'. [Online]. Available: http://www.itu.int/en/ITUT/techwatch/Pages/tactile-internet.aspx. [Accessed: 05-Apr-2017].

[3] M. Jinno, T. Ohara, Y. Sone, A. Hirano, O. Ishida, and M. Tomizawa, 'Elastic and adaptive optical networks: possible adoption scenarios and future standardization aspects', IEEE Commun. Mag., vol. 49, no. 10, pp. 164-172, Oct. 2011.

[4] T. Koonen, 'Fiber to the Home/Fiber to the Premises: What, Where, and When?', Proc. IEEE, vol. 94, no. 5, pp. 911-934, May 2006.

[5] C.-H. Lee, W. V. Sorin, and B. Y. Kim, 'Fiber to the Home Using a PON Infrastructure', J. Light. Technol., vol. 24, no. 12, pp. 4568-4583, Dec. 2006.

[6] H. Beyranvand and J. Salehi, 'Multirate and multi-quality-of-service passive optical network based on hybrid WDM/OCDM system', IEEE Commun. Mag., vol. 49, no. 2, pp. s39-s44, Feb. 2011.

[7] A. Dhaini, P.-H. Ho, and G. Shen, 'Toward green next-generation passive optical networks', IEEE Commun. Mag., vol. 49, no. 11, pp. 94-101, Nov. 2011.

[8] K.-I. Kitayama et al., '40G-OCDMA-PON System With an Asymmetric Structure Using a Single Multi-Port and Sampled SSFBG Encoder/Decoders', J. Light. Technol. Vol. 32, Issue 6, pp. 1132-1143, vol. 32, no. 6, pp. 1132-1143, 2014.

[9] K. Kitayama, 'Rationale of OCDMA/OFDMA for NG-PON', in 2011 ICO International Conference on Information Photonics, 2011, pp. 1-2.

[10] K. Kitayama, Xu Wang, and Naoya Wada, 'OCDMA over WDM PONsolution path to gigabit-symmetric FTTH', J. Light. Technol., vol. 24, no. 4, pp. 1654-1662, Apr. 2006. 


\section{BIBLIOGRAPHY}

[11] R. Davey, J. Kani, F. Bourgart, and K. McCammon, 'Options for future optical access networks', IEEE Commun. Mag., vol. 44, no. 10, pp. 50-56, Oct. 2006.

[12] Y. Luo et al., 'Time-and Wavelength-Division Multiplexed Passive Optical Network (TWDM-PON) for Next-Generation PON Stage 2 (NG-PON2)', J. Light. Technol., vol. 31, no. 4, pp. 587-593, 2013.

[13] H. Ghafouri-Shiraz and M. M. Karbassian, Optical CDMA networks: principles, analysis, and applications. Wiley, 2012.

[14] K. Fouli and M. Maier, 'OCDMA and Optical Coding: Principles, Applications, and Challenges [Topics in Optical Communications]', IEEE Commun. Mag., vol. 45, no. 8, pp. 27-34, Aug. 2007.

[15] A. Stok and E. H. Sargent, 'The role of optical CDMA in access networks', IEEE Commun. Mag., vol. 40, no. 9, pp. 83-87, Sep. 2002.

[16] K.-I. Kitayama, Optical code division multiple access : a practical perspective. Cambridge University Press, 2014.

[17] H. Beyranvand and J. A. Salehi, 'Multiservice Provisioning and Quality of Service Guarantee in WDM Optical Code Switched GMPLS Core Networks', J. Light. Technol. Vol. 27, Issue 12, pp. 1754-1762, vol. 27, no. 12, pp. 1754-1762, 2009.

[18] H. Beyranvand and J. A. Salehi, 'Multi-service path switching in alloptical GMPLS core network', in 2008 International Symposium on Telecommunications, 2008, pp. 494-498.

[19] P. Prucnal, M. Santoro, and Ting Fan, 'Spread spectrum fiber-optic local area network using optical processing', J. Light. Technol., vol. 4, no. 5, pp. 547-554, 1986.

[20] A. M. Weiner, J. P. Heritage, and J. A. Salehi, 'Encoding and decoding of femtosecond pulses', Opt. Lett., vol. 13, no. 4, p. 300, Apr. 1988.

[21] J. A. Salehi, 'Code division multiple-access techniques in optical fiber networks. I. Fundamental principles', IEEE Trans. Commun., vol. 37, no. 8, pp. 824-833, 1989. 
[22] J. A. Salehi and C. A. Brackett, 'Code division multiple-access techniques in optical fiber networks. II. Systems performance analysis', IEEE Trans. Commun., vol. 37, no. 8, pp. 834-842, 1989.

[23] F. R. K. Chung, J. A. Salehi, and V. K. Wei, 'Optical Orthogonal Codes: Design, Analysis, and Applications', IEEE Transactions on Information Theory, vol. 35, no. 3. pp. 595-604, 1989.

[24] Jun-Jie Chen and Guu-Chang Yang, 'CDMA fiber-optic systems with optical hard limiters', J. Light. Technol., vol. 19, no. 7, pp. 950-958, Jul. 2001.

[25] X. Lu and R. T. Chen, 'Polymeric Optical Code-Division Multiple-Access (CDMA) Encoder and Decoder Modules', Polymers (Basel)., vol. 3, no. 4, pp. 1554-1564, Sep. 2011.

[26] B. Yeh, C. Lin, and J. Wu, 'Noncoherent Spectral / Time / Spatial Optical CDMA System Using 3-D Perfect Difference Codes', J. Light. Technol., vol. 27, no. 6, pp. 744-759, Mar. 2009.

[27] I. Glesk, Y. K. Huang, C. S. Brès, and P. R. Prucnal, 'Design and demonstration of a novel optical CDMA platform for use in avionics applications', Opt. Commun., vol. 271, no. 1, pp. 65-70, 2007.

[28] F. Akhoundi, M. V. Jamali, N. B. Hassan, H. Beyranvand, A. Minoofar, and J. A. Salehi, 'Cellular Underwater Wireless Optical CDMA Network: Potentials and Challenges', IEEE Access, vol. 4, pp. 4254-4268, 2016.

[29] X. Wang, K. Matsushima, A. Nishiki, N. Wada, and K. Kitayama, 'High reflectivity superstructured FBG for coherent optical code generation and recognition', Opt. Express, vol. 12, no. 22, p. 5457, 2004.

[30] X. Wang, K. Matsushima, K. Kitayama, A. Nishiki, N. Wada, and F. Kubota, 'High-performance optical code generation and recognition by use of a 511-chip, 640-Gchip/s phase-shifted superstructured fiber Bragg grating', Opt. Lett., vol. 30, no. 4, p. 355, Feb. 2005.

[31] Naoki Nakagawa et al., 'Experimental demonstration of secure 16-ary, 2.5Gbit/s OCDMA using single multi-port en/decoder', in European Conference on Optical Communication, 2008. 


\section{BIBLIOGRAPHY}

[32] Xu Wang, Naoya Wada, G. Cincotti, T. Miyazaki, and K. Kitayama, 'Demonstration of over 128-gb/s-capacity (12-User/spl times/10.71$\mathrm{gb} / \mathrm{s} / \mathrm{user}$ ) asynchronous OCDMA using FEC and AWG-based multiport optical encoder/decoders', IEEE Photonics Technol. Lett., vol. 18, no. 15, pp. 1603-1605, Aug. 2006.

[33] X. Wang, T. Hamanaka, N. Wada, and K. Kitayama, 'Dispersionflattened-fiber based optical thresholder for multiple-access-interference suppression in OCDMA system', Opt. Express, vol. 13, no. 14, p. 5499, 2005.

[34] G. Cincotti, N. Wada, and K. Kitayama, 'Characterization of a Full Encoder/Decoder in the AWG Configuration for Code-Based Photonic Routers-Part I: Modeling and Design', J. Light. Technol., vol. 24, no. 1, p. 103, 2006.

[35] Xu Wang and Naoya Wada, 'Experimental demonstration of OCDMA traffic over optical packet switching network with hybrid PLC and SSFBG en/decoders', J. Light. Technol., vol. 24, no. 8, pp. 3012-3020, Aug. 2006.

[36] N. Kataoka, G. Cincotti, N. Wada, and K. Kitayama, ‘Demonstration of asynchronous, 40Gbps x 4-user DPSK-OCDMA transmission using a multi-port encoder/decoder', Opt. Express, vol. 19, no. 26, p. B965, Dec. 2011.

[37] S. Yoshima et al., '10 Gb/s-Based PON Over OCDMA Uplink Burst Transmission Using SSFBG Encoder/Multi-Port Decoder and Burst-Mode Receiver', J. Light. Technol., vol. 28, no. 4, pp. 365-371, Feb. 2010.

[38] J. A. Salehi, 'Emerging optical CDMA techniques and applications', Int. J. Opt. Photonics, vol. 1, no. 1, pp. 15-32, 2007.

[39] T. R. Raddo, A. L. Sanches, I. Tafur Monroy, and B.-H. V. Borges, 'Throughput Performance Evaluation of Multiservice Multirate OCDMA in Flexible Networks', IEEE Photonics J., vol. 8, no. 1, pp. 1-15, Feb. 2016.

[40] H. Fathallah, L. A. Rusch, and S. LaRochelle, 'Passive optical fast frequency-hop CDMA communications system', J. Light. Technol., vol. 17, no. 3, pp. 397-405, Mar. 1999. 
[41] B. Ghaffari and J. Salehi, 'Multiclass, Multistage, and Multilevel FiberOptic CDMA Signaling Techniques Based on Advanced Binary Optical Logic Gate Elements', IEEE Trans. Commun., vol. 57, no. 5, pp. 1424-1432, May 2009.

[42] N. G. Tarhuni, T. O. Korhonen, E. Mutafungwa, and M. S. Elmusrati, 'Multiclass optical orthogonal codes for multiservice optical CDMA networks', J. Light. Technol., vol. 24, no. 2, pp. 694-704, Feb. 2006.

[43] E. K. H. Ng and E. H. Sargent, 'Optimum threshold detection in real-time scalable high-speed multi-wavelength optical code-division multipleaccess LANs', IEEE Trans. Commun., vol. 50, no. 5, pp. 778-784, May 2002.

[44] S. Sahuguede, A. Julien-Vergonjanne, and J.-P. Cances, 'Performance of OCDMA system with FEC based on interference statistical distribution analysis', Eur. Trans. Telecommun., vol. 21, no. 3, pp. 276-287, 2009.

[45] S. V. Maric, O. Moreno, and C. J. Corrada, 'Multimedia transmission in fiber-optic LANs using optical CDMA', J. Light. Technol., vol. 14, no. 10, pp. 2149-2153, Oct. 1996.

[46] C. K. See, C. K. See, Z. F. Ghassemlooy, J. M. Holding, and R. Mclaughlin, 'Comparison of binomial and gaussian distributions for evaluating optical DS-CDMA system BER performance.', Proceeding PREP, pp. 13-14, 2003.

[47] E. Inaty, H. M. H. Shalaby, P. Fortier, and L. A. Rusch, 'Multirate optical fast frequency hopping CDMA system using power control', J. Light. Technol., vol. 20, no. 2, pp. 166-177, 2002.

[48] W. C. Kwong, P. A. Perrier, and P. R. Prucnal, 'Performance comparison of asynchronous and synchronous code-division multiple-access techniques for fiber-optic local area networks', IEEE Trans. Commun., vol. 39, no. 11, pp. 1625-1634, 1991.

[49] Lim Nguyen, J. F. Young, and Behnaam Aazhang, 'Photoelectric current distribution and bit error rate in optical communication systems using a superfluorescent fiber source', J. Light. Technol., vol. 14, no. 6, pp. 14551466, Jun. 1996. 


\section{BIBLIOGRAPHY}

[50] K. B. Letaief, 'The performance of optical fiber direct-sequence spreadspectrum multiple-access communications systems', IEEE Trans. Commun., vol. 43, no. 11, pp. 2662-2666, 1995.

[51] I. Andonovic, L. Tančevski, J. Budin, and M. Tur, 'Hybrid wavelength hopping/time spreading code division multiple access systems', IEE Proc. - Optoelectron., vol. 143, no. 3, pp. 161-166, Jun. 1996.

[52] L. Tancevski and I. Andonovic, 'Hybrid wavelength hopping/time spreading schemes for use in massive optical networks with increased security', J. Light. Technol., vol. 14, no. 12, pp. 2636-2647, 1996.

[53] Sun Shurong, Hongxi Yin, Ziyu Wang, and Anshi Xu, 'A new family of 2D optical orthogonal codes and analysis of its performance in optical CDMA access networks', J. Light. Technol., vol. 24, no. 4, pp. 1646-1653, Apr. 2006.

[54] S. Zou, M. M. Karbassian, and H. Ghafouri-Shiraz, 'Extended 2D Codes Supporting Multirate and QoS in Optical CDMA Networks With Poisson and Binomial MAI Models', J. Opt. Commun. Netw., vol. 5, no. 5, p. 524, May 2013.

[55] H. Fathallah, 'Optical fast frequency hopping CDMA: principle, simulation and experiment', Thèse Présentée Fac. des Sci. Génie, Univ. Laval, p. 175, 2002.

[56] E. Inaty, P. Fortier, and L. A. Rusch, 'SIR performance evaluation of a multirate OFFH-CDMA system', IEEE Commun. Lett., vol. 5, no. 5, pp. 224-226, May 2001.

[57] M. A. Shoaie, S. Khazraei, and M. R. Pakravan, 'Performance Analysis of Slotted ALOHA Random Access Packet-Switching Optical CDMA Networks Using Generalized Optical Orthogonal Codes and M-ary Overlapping PPM Signaling', J. Opt. Commun. Netw., vol. 3, no. 7, p. 568, Jul. 2011.

[58] A. Stok and E. H. Sargent, 'Comparison of diverse optical CDMA codes using a normalized throughput metric', IEEE Commun. Lett., vol. 7, no. 5, pp. 242-244, May 2003. 
[59] F. R. Durand, M. S. Filho, and T. Abrão, 'The effects of power control on the optical CDMA random access protocol', Opt. Switch. Netw., vol. 9, no. 1, pp. 52-60, 2012.

[60] Cherng-Shung Hsu and V. O. K. Li, 'Performance analysis of slotted fiberoptic code-division multiple-access (CDMA) packet networks', IEEE Trans. Commun., vol. 45, no. 7, pp. 819-828, Jul. 1997.

[61] H. M. H. Shalaby, 'Optical CDMA Random Access Protocols With and Without Pretransmission Coordination', J. Light. Technol. Vol. 21, Issue 11, pp. 2455-, vol. 21, no. 11, p. 2455, 2003.

[62] Jin Young Kim and H. V. Poor, 'Turbo-coded packet transmission for an optical CDMA network', J. Light. Technol., vol. 18, no. 12, pp. 1905-1916, 2000.

[63] S. Khaleghi, S. Khaleghi, and K. Jamshidi, 'Performance Analysis of a Spectrally Phase-Encoded Optical Code Division Multiple Access Packet Network', J. Opt. Commun. Netw., vol. 1, no. 3, p. 213, Aug. 2009.

[64] N. Karafolas and D. Uttamchandani, 'Optical Fiber Code Division Multiple Access Networks: A Review', Opt. Fiber Technol., vol. 2, no. 2, pp. 149-168, Apr. 1996.

[65] A. L. Sanches, T. R. Raddo, J. V. dos Reis, and B.-H. V. Borges, 'Performance Analysis of Single and Multirate FFH-OCDMA Networks Based on PSK Modulation Formats', J. Opt. Commun. Netw., vol. 7, no. 11, p. 1084, Nov. 2015.

[66] T. H. Shake, 'Security performance of optical CDMA Against eavesdropping', J. Light. Technol., vol. 23, no. 2, pp. 655-670, Feb. 2005.

[67] H. S. Chung, S. H. Chang, B. K. Kim, and K. Kim, 'Experimental demonstration of security-improved OCDMA scheme based on incoherent broadband light source and bipolar coding', Opt. Fiber Technol., vol. 14, no. 2, pp. 130-133, Apr. 2008.

[68] D. E. Leaird, Z. Jiang, and A. M. Weiner, 'Experimental investigation of security issues in OCDMA: a code-switching scheme', Electron. Lett., vol. 41 , no. 14, p. $817,2005$. 


\section{BIBLIOGRAPHY}

[69] H. Y. Choi, T. Tsuritani, and I. Morita, 'BER-adaptive flexible-format transmitter for elastic optical networks', Opt. Express, vol. 20, no. 17, p. 18652, Aug. 2012.

[70] G. Mazzini, G. Setti, and R. Rovatti, 'Chaotic complex spreading sequences for asynchronous DS-CDMA. I. System modeling and results', IEEE Trans. Circuits Syst. I Fundam. Theory Appl., vol. 44, no. 10, pp. 937947, 1997.

[71] K. Umeno and K. Kitayama, 'Spreading sequences using periodic orbits of chaos for CDMA', Electron. Lett., vol. 35, no. 7, p. 545, 1999.

[72] X. Wang and K. Kitayama, 'Analysis of Beat Noise in Coherent and Incoherent Time-Spreading OCDMA', J. Light. Technol., vol. 22, no. 10, pp. 2226-2235, Oct. 2004.

[73] F. R. K. Chung, J. A. Salehi, and V. K. Wei, 'Optical Orthogonal Codes: Design, Analysis, and Applications', IEEE Trans. Inf. Theory, vol. 35, no. 3, pp. 595-604, May 1989.

[74] A. A. Shaar and P. A. Davies, 'Prime sequences: quasi-optimal sequences for OR channel code division multiplexing', Electron. Lett., vol. 19, no. 21, p. 888, 1983.

[75] I. Andonovic and L. Tancevski, 'Wavelength hopping/time spreading code division multiple access systems', Electron. Lett., vol. 30, no. 17, pp. 1388-1390, Aug. 1994.

[76] H. Ben Jaafar, S. LaRochelle, P.-Y. Cortes, and H. Fathallah, '1.25 Gbit/s transmission of optical FFH-OCDMA signals over $80 \mathrm{~km}$ with 16 users', in OFC 2001. Optical Fiber Communication Conference and Exhibit. Technical Digest Postconference Edition (IEEE Cat. 01CH37171), vol. 2, p. TuV3-1TuV3-3.

[77] Sheng Peng Wan and $\mathrm{Yu} \mathrm{Hu}$, 'Two-dimensional optical CDMA differential system with prime/OOC codes', IEEE Photonics Technol. Lett., vol. 13, no. 12, pp. 1373-1375, Dec. 2001.

[78] Guu-Chang Yang and W. C. Kwong, 'Performance comparison of multiwavelength CDMA and WDMA+CDMA for fiber-optic networks', IEEE Trans. Commun., vol. 45, no. 11, pp. 1426-1434, 1997. 
[79] L. Galdino, T. R. Raddo, A. L. Sanches, L. H. Bonani, and E. Moschim, 'Performance comparison of hybrid 1-D WDM/OCDMA and 2-D OCDMA towards future access network migration scenario', in 2012 14th International Conference on Transparent Optical Networks (ICTON), 2012, pp. $1-4$.

[80] A. L. Sanches, J. V. dos Reis, and B.-H. V. Borges, 'Analysis of High-Speed Optical Wavelength/Time CDMA Networks Using Pulse-Position Modulation and Forward Error Correction Techniques', J. Light. Technol., vol. 27, no. 22, pp. 5134-5144, Nov. 2009.

[81] H. Yin, W. Liang, and L. Ma, 'Construction and performance analysis of 2-D variable-length variable-weight optical orthogonal codes', Photonic Netw. Commun., vol. 18, no. 1, pp. 122-128, Aug. 2009.

[82] K. O. Hill and G. Meltz, 'Fiber Bragg grating technology fundamentals and overview', J. Light. Technol., vol. 15, no. 8, pp. 1263-1276, 1997.

[83] E. Inaty, H. M. H. Shalaby, and P. Fortier, 'On the Cutoff Rates of a Multiclass OFFH-CDMA System', IEEE Trans. Commun., vol. 53, no. 2, pp. 323-334, Feb. 2005.

[84] Li Bin, 'One-coincidence sequences with specified distance between adjacent symbols for frequency-hopping multiple access', IEEE Trans. Commun., vol. 45, no. 4, pp. 408-410, Apr. 1997.

[85] E. Inaty, P. Fortier, and L. A. Rusch, 'SIR performance evaluation of a multirate OFFH-CDMA system', IEEE Commun. Lett., vol. 5, no. 5, pp. 224-226, 2001.

[86] A. J. Mendez, R. M. Gagliardi, V. J. Hernandez, C. V. Bennett, and W. J. Lennon, 'High-Performance Optical CDMA System Based on 2-D Optical Orthogonal Codes', J. Light. Technol., vol. 22, no. 11, pp. 2409-2419, Nov. 2004.

[87] Cheng-Yuan Chang, Guu-Chang Yang, and W. C. Kwong, 'Wavelengthtime codes with maximum cross-correlation function of two for multicode-keying optical CDMA', J. Light. Technol., vol. 24, no. 3, pp. 1093-1100, Mar. 2006. 
[88] A. R. Forouzan, M. Nasiri-Kenari, and N. Rezaee, 'Frame Time-Hopping Patterns in Multirate Optical CDMA Networks Using Conventional and Multicode Schemes', IEEE Trans. Commun., vol. 53, no. 5, pp. 863-875, May 2005.

[89] E. Narimanov, W. C. Kwong, G.-C. Yang, and P. R. Prucnal, 'Shifted Carrier-Hopping Prime Codes for Multicode Keying in Wavelength-Time O-CDMA', IEEE Trans. Commun., vol. 53, no. 12, pp. 2150-2156, Dec. 2005.

[90] T. Ohtsuki, 'Performance of multicode direct-detection optical CDMA systems', in Global Telecommunications Conference, GLOBECOM, 1998, vol. 6, pp. 3227-3232.

[91] M. Saito, H. Okada, T. Sato, T. Yamazato, M. Katasyama, and A. Ogawa, 'Throughput improvement of CDMA slotted ALOHA system by modified channel load sensing protocol', in Proceedings of PIMRC '96 - 7th International Symposium on Personal, Indoor, and Mobile Communications, vol. 1, pp. 103-107.

[92] Hung-Wei Chen, Guu-Chang Yang, Cheng-Yuan Chang, Tsung-Chi Lin, and W. C. Kwong, 'Spectral Efficiency Study of Two Multirate Schemes for Asynchronous Optical CDMA', J. Light. Technol., vol. 27, no. 14, pp. 2771-2778, Jul. 2009.

[93] A. E. Farghal, H. M. H. Shalaby, K. Kato, and R. K. Pokharel, 'Optical Code-Division Multiplexing (OCDM) Networks Adopting Code-Shift Keying/Overlapping PPM Signaling: Proposal and Performance Analysis', IEEE Trans. Commun., vol. 63, no. 10, pp. 3779-3788, Oct. 2015.

[94] A. E. Farghal, H. M. H. Shalaby, and Z. Kawasaki, 'Multirate Multiservice All-Optical Code Switched GMPLS Core Network Utilizing Multicode Variable-Weight Optical Code-Division Multiplexing', J. Opt. Commun. Netw., vol. 6, no. 8, p. 670, Aug. 2014.

[95] T. R. Raddo, A. L. Sanches, J. V. dos Reis, and B.-H. V. Borges, 'A New Approach for Evaluating the BER of a Multirate, Multiclass OFFH-CDMA System', IEEE Commun. Lett., vol. 16, no. 2, pp. 259-261, Feb. 2012.

[96] T. R. Raddo, A. Sanches, J. V. dos Reis Jr, and B.-H. V. Borges, 'Influence of the MAI Distribution over the BER Evaluation in a Multirate, Multiclass OOC-OCDMA System', in Advanced Photonics, 2011, p. ATuB5. 
[97] H. Beyranvand, B. M. Ghaffari, and J. A. Salehi, 'Multirate, DifferentiatedQoS, and Multilevel Fiber-Optic CDMA System via Optical Logic Gate Elements', J. Light. Technol., vol. 27, no. 19, pp. 4348-4359, Oct. 2009.

[98] H. Lundqvist and G. Karlsson, 'On error-correction coding for CDMA PON', J. Light. Technol., vol. 23, no. 8, pp. 2342-2351, Aug. 2005.

[99] M. M. Karbassian and H. Ghafouri-Shiraz, 'IP Routing and Transmission Analysis in Optical CDMA Networks: Coherent Modulation With Incoherent Demodulation', J. Light. Technol., vol. 27, no. 17, pp. 3845-3852, Sep. 2009.

[100] M. M. Karbassian and H. Ghafouri-Shiraz, 'IP Routing and Traffic Analysis in Coherent Optical CDMA Networks', J. Light. Technol., vol. 27, no. 10, pp. 1262-1268, May 2009.

[101] T. R. Raddo, A. L. Sanches, I. T. Monroy, and B.-H. V. Borges, 'Multirate IP traffic transmission in flexible access networks based on optical FFHCDMA', in 2016 IEEE International Conference on Communications (ICC), 2016, pp. 1-6.

[102] D. Raychaudhuri, 'Performance Analysis of Random Access PacketSwitched Code Division Multiple Access Systems', IEEE Trans. Commun., vol. 29, no. 6, pp. 895-901, Jun. 1981.

[103] M. A. Shoaie, S. Khazraei, and M. R. Pakravan, 'Performance Analysis of Slotted ALOHA Random Access Packet-Switching Optical CDMA Networks Using Generalized Optical Orthogonal Codes and M-ary Overlapping PPM Signaling', J. Opt. Commun. Netw., vol. 3, no. 7, p. 568, Jul. 2011.

[104] R. Raad, E. Inaty, P. Fortier, and H. M. H. Shalaby, 'Optical SALOHA/CDMA systems for multirate applications: architecture, performance evaluation, and system stability', J. Light. Technol., vol. 24, no. 5, pp. 1968-1977, May 2006.

[105] T. R. Raddo, A. L. Sanches, J. V. dos Reis, and B.-H. V. Borges, 'Throughput evaluation of multirate, multiservice 2-D OCDMA packet networks', in 2014 16th International Conference on Transparent Optical Networks (ICTON), 2014, pp. 1-4. 
[106] T. R. Raddo, A. L. Sanches, B.-H. V. Borges, and I. T. Monroy, 'Throughput performance analysis of multirate, multiclass S-ALOHA OFFH-CDMA packet networks', in 2015 17th International Conference on Transparent Optical Networks (ICTON), 2015, pp. 1-4.

[107] A. Carleial and M. Hellman, 'Bistable Behavior of ALOHA-Type Systems', IEEE Trans. Commun., vol. 23, no. 4, pp. 401-410, Apr. 1975.

[108] E. Narimanov, W. C. Kwong, G.-C. Yang, and P. R. Prucnal, 'Shifted Carrier-Hopping Prime Codes for Multicode Keying in Wavelength-Time O-CDMA', IEEE Trans. Commun., vol. 53, no. 12, pp. 2150-2156, Dec. 2005.

[109] T. R. Raddo, A. L. Sanches, I. T. Monroy, and B.-H. V. Borges, 'Packet throughput performance of multiservice, multirate OCDMA in elastic networks', in 2016 IEEE International Conference on Communications (ICC), 2016, pp. 1-6.

[110] Mohamed, Shalaby, and El-Badawy, 'Performance analysis of an optical CDMA MAC protocol with variable-size sliding window', J. Light. Technol., vol. 24, no. 10, pp. 3590-3597, Oct. 2006.

[111] A. L. Sanches, T. R. Raddo, J. V. dos Reis, and B.-H. V. Borges, 'Highly efficient FFH-OCDMA packet network with coherent advanced modulation formats', in 2014 16th International Conference on Transparent Optical Networks (ICTON), 2014, pp. 1-4.

[112] Li Bin, 'One-coincidence sequences with specified distance between adjacent symbols for frequency-hopping multiple access', IEEE Trans. Commun., vol. 45, no. 4, pp. 408-410, Apr. 1997.

[113] D. C. Montgomery and G. C. Runger, Applied statistics and probability for engineers. Wiley, 2011.

[114] G. E. P. Box, J. S. Hunter, and W. G. Hunter, Statistics for experimenters: design, innovation, and discovery. Wiley-Interscience, 2005.

[115] G.-H. Gho and J. M. Kahn, 'Rate-Adaptive Modulation and Coding for Optical Fiber Transmission Systems', J. Light. Technol., vol. 30, no. 12, pp. 1818-1828, Jun. 2012. 
[116] M. Virte, K. Panajotov, H. Thienpont, and M. Sciamanna, 'Deterministic polarization chaos from a laser diode', Nat. Photonics, vol. 7, no. 1, pp. 16, 2012.

[117] M. Virte, 'Two-mode dynamics and switching in quantum dot lasers.', PhD Thesis, Vrije Univ. Brussels, 2014.

[118] Y. Lau, 'Techniques in secure chaos communication', PhD Thesis, RMIT Univ., 2006.

[119] H. P. Ren, M. S. Baptista, and C. Grebogi, 'Wireless communication with chaos', Phys. Rev. Lett., vol. 110, no. 18, pp. 1-5, 2013.

[120] V. Annovazzi-Lodi, G. Aromataris, M. Benedetti, and S. Merlo, 'Secure chaotic transmission on a free-space optics data link', IEEE J. Quantum Electron., vol. 44, no. 11, pp. 1089-1095, 2008.

[121] A. Argyris et al., 'Chaos-based communications at high bit rates using commercial fibre-optic links.', Nature, vol. 438, no. 7066, pp. 343-346, 2005.

[122] N. Jiang, J. Wang, D. Liu, C. Xue, and K. Qiu, 'Secure WDM-PON based on chaos synchronization and subcarrier modulation multiplexing', $J$. Opt. Soc. Am. B, vol. 33, no. 4, p. 637, Apr. 2016.

[123] L. M. Pecora and T. L. Carroll, 'Synchronization in chaotic systems', Phys. Rev. Lett., vol. 64, no. 8, pp. 821-824, Feb. 1990.

[124] K. M. Cuomo, A. V. Oppenheim, and S. H. Strogatz, 'Synchronization of Lorenz-based chaotic circuits with applications to communications', IEEE Trans. Circuits Syst. II Analog Digit. Signal Process., vol. 40, no. 10, pp. 626633, 1993.

[125] J. Mork, B. Tromborg, and J. Mark, 'Chaos in semiconductor lasers with optical feedback: theory and experiment', IEEE J. Quantum Electron., vol. 28, no. 1, pp. 93-108, 1992.

[126] D. Kanakidis, A. Argyris, and D. Syvridis, 'Performance characterization of high-bit-rate optical chaotic communication systems in a back-to-back configuration', J. Light. Technol., vol. 21, no. 3, pp. 750-758, Mar. 2003. 


\section{BIBLIOGRAPHY}

[127] Jia-ming Liu, How-foo Chen, and Shuo Tang, 'Synchronized chaotic optical communications at high bit rates', IEEE J. Quantum Electron., vol. 38, no. 9, pp. 1184-1196, Sep. 2002.

[128] 'Designing With VCSELs'. [Online]. Available: http://myvcsel.com/designing-with-vcsels/. [Accessed: 25-Apr-2017].

[129] K. Panajotov et al., 'Impact of in-plane anisotropic strain on the polarization behavior of vertical-cavity surface-emitting lasers', Appl. Phys. Lett., vol. 77, no. 11, p. 1590, 2000.

[130] B. Ryvkin et al., 'Effect of photon-energy-dependent loss and gain mechanisms on polarization switching in vertical-cavity surface-emitting lasers', J. Opt. Soc. Am. B, vol. 16, no. 11, p. 2106, Nov. 1999.

[131] A. K. Jansen van Doorn, M. P. van Exter, and J. P. Woerdman, 'Elastooptic anisotropy and polarization orientation of vertical-cavity surfaceemitting semiconductor lasers', Appl. Phys. Lett., vol. 69, no. 8, pp. 10411043, Aug. 1996.

[132] A. K. Jansen van Doorn, M. P. van Exter, and J. P. Woerdman, 'Tailoring the birefringence in a vertical-cavity semiconductor laser', Appl. Phys. Lett., vol. 69, no. 24, pp. 3635-3637, Dec. 1996.

[133] M. Lindemann, T. Pusch, R. Michalzik, N. C. Gerhardt, and M. R. Hofmann, 'Frequency tuning of polarization oscillations: Toward highspeed spin-lasers', Appl. Phys. Lett., vol. 108, no. 4, 2016.

[134] T. Pusch, M. Lindemann, N. C. Gerhardt, and M. R. Hofmann, 'Verticalcavity surface-emitting lasers with birefringence splitting above 250 GHz', Electron. Lett., vol. 51, no. 20, pp. 1600-1602, 2015.

[135] M. Virte, K. Panajotov, and M. Sciamanna, 'Bifurcation to nonlinear polarization dynamics and chaos in vertical-cavity surface-emitting lasers', Phys. Rev. A, vol. 87, no. 1, p. 13834, 2013.

[136] M. Sondermann, M. Weinkath, T. Ackemann, J. Mulet, and S. Balle, 'Twofrequency emission and polarization dynamics at lasing threshold in vertical-cavity surface-emitting lasers', Phys. Rev. A, vol. 68, no. 3, p. 33822, Sep. 2003. 
[137] M. Virte, M. Sciamanna, E. Mercier, and K. Panajotov, 'Bistability of timeperiodic polarization dynamics in a free-running VCSEL', Opt. Express, vol. 22, no. 6, p. 6772, 2014.

[138] L. Olejniczak et al., 'Polarization switching and polarization mode hopping in quantum dot vertical-cavity surface-emitting lasers.', Opt. Express, vol. 19, no. 3, pp. 2476-84, 2011.

[139] M. B. Willemsen, M. U. F. Khalid, M. P. van Exter, and J. P. Woerdman, 'Polarization Switching of a Vertical-Cavity Semiconductor Laser as a Kramers Hopping Problem', Phys. Rev. Lett., vol. 82, no. 24, p. 4815, Jun. 1999.

[140] M. Virte, E. Mirisola, M. Sciamanna, and K. Panajotov, 'Asymmetric dwell-time statistics of polarization chaos from free-running VCSEL', Opt. Lett., vol. 40, no. 8, pp. 1865-1868, 2015.

[141] A. Wolf, J. B. Swift, H. L. Swinney, and J. A. Vastano, 'Determining Lyapunov exponents from a time series', Phys. D Nonlinear Phenom., vol. 16, no. 3, pp. 285-317, Jul. 1985.

[142] M. Virte, E. Mercier, H. Thienpont, K. Panajotov, and M. Sciamanna, 'Physical random bit generation from chaotic solitary laser diode', Opt. Express, vol. 22, no. 14, p. 17271, 2014.

[143] K. Fraedrich and R. Wang, 'Estimating the correlation dimension of an attractor from noisy and small datasets based on re-embedding', Phys. $D$ Nonlinear Phenom., vol. 65, no. 4, pp. 373-398, Jun. 1993.

[144] C. J. Chang-Hasnain, J. P. Harbison, G. Hasnain, A. C. Von Lehmen, L. T. Florez, and N. G. Stoffel, 'Dynamic, polarization, and transverse mode characteristics of vertical cavity surface emitting lasers', IEEE J. Quantum Electron., vol. 27, no. 6, pp. 1402-1409, Jun. 1991. 